\title{
The Schrödinger model for the minimal representation of the indefinite orthogonal group $O(p, q)$
}

\author{
Toshiyuki Kobayashi* and Gen Mano
}

July 18, 2008

\footnotetext{
* Partially supported by Grant-in-Aid for Scientific Research (B) (18340037), Japan Society for the Promotion of Science.

2000 Mathematics Subject Classification. Primary 22E30; Secondary 22E46, $43 \mathrm{~A} 80$

Key words and phrases: minimal representation, Schrödinger model, generalization of the Fourier transform, Weil representation, indefinite orthogonal group, unitary representation, isotropic cone, Bessel functions, Meijer's $G$-functions
} 


\section{Contents}

1 Introduction $\quad 1$

1.1 Differential operators on the isotropic cone . . . . . . . . 4

1.2 'Fourier transform' $\mathcal{F}_{C}$ on the isotropic cone $C \ldots \ldots$

1.3 Kernel of $\mathcal{F}_{C}$ and Bessel distributions . . . . . . . . . . 13

1.4 Perspectives from representation theory - finding smallest objects ......................... 17

1.5 Minimal representations of simple Lie groups . . . . . . . 18

1.6 Schrödinger model for the Weil representation . . . . . . . . . 20

1.7 Schrödinger model for the minimal representation of $O(p, q)$. 21

1.8 Uncertainty relation - inner products and $G$-actions . . . . . 26

1.9 Special functions and minimal representations . . . . . . . . 30

1.10 Organization of this book . . . . . . . . . . . 33

1.11 Acknowledgements . . . . . . . . . . . . . . 34

2 Two models of the minimal representation of $O(p, q) \quad 35$

2.1 Conformal model . . . . . . . . . . . . . . . . 36

$2.2 \quad L^{2}$-model (the Schrödinger model) . . . . . . . . . . . 40

2.3 Lie algebra action on $L^{2}(C) \ldots \ldots \ldots \ldots . \ldots \ldots$

2.4 Commuting differential operators on $C \ldots \ldots \ldots . . . . .48$

2.5 The unitary inversion operator $\mathcal{F}_{C}=\pi\left(w_{0}\right) \ldots \ldots \ldots$

$3 \quad K$-finite eigenvectors in the Schrödinger model $L^{2}(C) \quad 65$

3.1 Result of this chapter . . . . . . . . . . . 65

$3.2 K \cap M^{\max }$-invariant subspaces $H_{l, k} \ldots \ldots \ldots \ldots \ldots$

3.3 Integral formula for the $K \cap M^{\max }$-intertwiner $\ldots \ldots . . .70$

$3.4 \quad K$-finite vectors $f_{l, k}$ in $L^{2}(C) \ldots \ldots \ldots \ldots 71$

3.5 Proof of Theorem 3.1.1. . . . . . . . . . . . . 73 
\begin{tabular}{ll|l}
4 & Radial part of the inversion & $\mathbf{7 6}$
\end{tabular}

4.1 Result of this chapter . . . . . . . . . . . . 76

4.2 Proof of Theorem $4.1 .1(1) \quad \ldots \ldots \ldots$. . . . . . . . . 81

4.3 Preliminary results on multiplier operators . . . . . . . . . . 81

4.4 Reduction to Fourier analysis . . . . . . . . . . . . 85

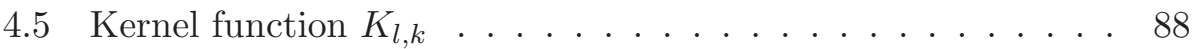

4.6 Proof of Theorem $4.1 .1(2) \quad \ldots \ldots \ldots 4$

5 Main theorem $\quad 99$

5.1 Result of this chapter . . . . . . . . . . . . . . . . . 99

5.2 Radon transform for the isotropic cone $C$. . . . . . . . . 101

5.3 Spectra of $K^{\prime}$-invariant operators on $S^{p-2} \times S^{q-2} \ldots \ldots . .104$

5.4 Proof of Theorem 5.1.1. . . . . . . . . . . . . . . . . . . 109

5.5 Proof of Lemma 5.4 .2 (Hermitian case $q=2) \ldots \ldots$

5.6 Proof of Lemma $5.4 .2(p, q>2) \ldots \ldots$

6 Bessel distributions $\quad 113$

6.1 Meijer's G-distributions . . . . . . . . . . . . . . . 113

6.2 Integral expression of Bessel distributions . . . . . . . . . . 119

6.3 Differential equations for Bessel distributions . . . . . . . 126

7 Appendix: special functions $\quad 132$

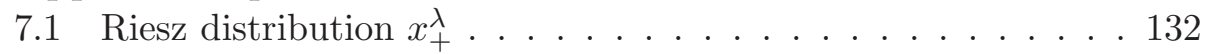

7.2 Bessel functions $J_{\nu}, I_{\nu}, K_{\nu}, Y_{\nu} \ldots \ldots \ldots \ldots \ldots$

7.3 Associated Legendre functions $P_{\nu}^{\mu} \ldots \ldots$. . . . . . . . 140

7.4 Gegenbauer polynomials $C_{l}^{\mu} \ldots \ldots \ldots \ldots$. . . . . . 141

7.5 Spherical harmonics $\mathcal{H}^{j}\left(\mathbb{R}^{m}\right)$ and branching laws . . . . . . 143

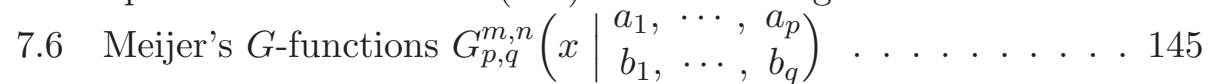

7.7 Appell's hypergeometric functions $F_{1}, F_{2}, F_{3}, F_{4} \ldots \ldots 151$

7.8 Hankel transform with trigonometric parameters . . . . . 152

7.9 Fractional integral of two variables . . . . . . . . . . . . 154

List of Symbols . . . . . . . . . . . . . . . . . . 163

Index . . . . . . . . . . . . . . . . . . 165 


\begin{abstract}
We introduce a generalization of the Fourier transform, denoted by $\mathcal{F}_{C}$, on the isotropic cone $C$ associated to an indefinite quadratic form of signature $\left(n_{1}, n_{2}\right)$ on $\mathbb{R}^{n}\left(n=n_{1}+n_{2}\right.$ : even $)$. This transform is in some sense the unique and natural unitary operator on $L^{2}(C)$, as is the case with the Euclidean Fourier transform $\mathcal{F}_{\mathbb{R}^{n}}$ on $L^{2}\left(\mathbb{R}^{n}\right)$. Inspired by recent developments of algebraic representation theory of reductive groups, we shed new light on classical analysis on the one hand, and give the global formulas for the $L^{2}$-model of the minimal representation of the simple Lie group $G=O\left(n_{1}+1, n_{2}+1\right)$ on the other hand.

The transform $\mathcal{F}_{C}$ expands functions on $C$ into joint eigenfunctions of fundamental differential operators which are mutually commuting, selfadjoint, and of second order. We decompose $\mathcal{F}_{C}$ into the singular Radon transform and the Mellin-Barnes integral, find its distribution kernel, and establish the inversion and the Plancherel formula. The transform $\mathcal{F}_{C}$ reduces to the Hankel transform if $G$ is $O(n, 2)$ or $O(3,3) \approx S L(4, \mathbb{R})$.

The unitary operator $\mathcal{F}_{C}$ together with multiplications and translations coming from the conformal transformation group $C O\left(n_{1}, n_{2}\right) \ltimes \mathbb{R}^{n_{1}+n_{2}}$ generates the minimal representation of the indefinite orthogonal group $G$. Various different models of the same representation have been constructed by Kazhdan, Kostant, Binegar-Zierau, Gross-Wallach, Zhu-Huang, Torasso, Brylinski, and Kobayashi-Ørsted, and others. Among them, our model gives the global formula of the whole group action on the simple Hilbert space $L^{2}(C)$, and generalizes the classic Schrödinger model $L^{2}\left(\mathbb{R}^{n}\right)$ of the Weil representation. Here, $\mathcal{F}_{C}$ plays a similar role to $\mathcal{F}_{\mathbb{R}^{n}}$.

Yet another motif is special functions. Large group symmetries in the minimal representation yield functional equations of various special functions. We find explicit $K$-finite vectors on $L^{2}(C)$, and give a new proof of the Plancherel formula for Meijer's $G$-transforms.
\end{abstract}




\section{Chapter 1}

\section{Introduction}

This book is a continuation of a series of our research projects [43, 44, 46, 47, 48, 49. Our motif is to open up and develop geometric analysis of a single infinite dimensional representation, namely, the minimal representation $\pi$ of the indefinite (even) orthogonal group.

This representation is surprisingly rich in its different models, through which we have cross-fertilization and interactions with various areas of mathematics such as conformal geometry and the Yamabe operator, Fourier analysis, ultra-hyperbolic equations and their conserved quantities, the Kepler problem, holomorphic semigroups, and analysis on isotropic cones. Among them, this book is devoted to the $L^{2}$-model (Schrödinger model), for which the local formula was established in a previous paper [49] with B. Ørsted. The global formula of the whole group action is the subject of this book.

We have limited ourselves to the very representation $\pi$, although some of our results could be generalized to other settings by the ideas developed here. This is primarily because we believe that geometric analysis of this specific minimal representation is of interest in its own right, and might open up an unexpected direction of research bridging different fields of mathematics, as in the case of the Weil representation (e.g. [18, 35, 36, 38, 61]).

Bearing this in mind, we will not only

- formalize our main results by means of representation theory,

but also

- formalize our main results without group theory.

We have made effort to expound the theory in a self-contained fashion as much as possible. 
For $n=n_{1}+n_{2}$, we denote by $\mathbb{R}^{n_{1}, n_{2}}$ the Euclidean space $\mathbb{R}^{n}$ endowed with the flat pseudo-Riemannian structure

$$
d s^{2}=d x_{1}^{2}+\cdots+d x_{n_{1}}^{2}-d x_{n_{1}+1}^{2}-\cdots-d x_{n}^{2},
$$

and define the isotropic cone $C$ by

$$
C:=\left\{x \in \mathbb{R}^{n} \backslash\{0\}: x_{1}^{2}+\cdots+x_{n_{1}}^{2}-x_{n_{1}+1}^{2}-\cdots-x_{n}^{2}=0\right\} .
$$

In this book, we will introduce the 'Fourier transform' $\mathcal{F}_{C}$ on the isotropic cone $C$ for $n$ even. This transform $\mathcal{F}_{C}$ is in some sense the unique and natural unitary operator on $L^{2}(C)$, as it is the case with the Euclidean Fourier transform $\mathcal{F}_{\mathbb{R}^{n}}$ on $L^{2}\left(\mathbb{R}^{n}\right)$.

Here is a brief guide to the three motivations of this book, with emphasis on the role of the unitary operator $\mathcal{F}_{C}$.

The first motivation comes from analysis on the isotropic cone $C$ itself. Different from non-isotropic hypersurfaces (e.g. hyperboloids) in $\mathbb{R}^{n_{1}, n_{2}}$, the restriction of $d s^{2}$ to $C$ is degenerate, and we do not have a natural pseudoRiemannian structure on $C$. Consequently, there is no natural single operator on $C$ such as the Laplace-Beltrami operator. However, it turns out that there are commuting, self-adjoint, second order differential operators $P_{1}, \ldots, P_{n}$ that we call fundamental differential operators on $C$ satisfying the algebraic relation $P_{1}^{2}+\cdots+P_{n_{1}}^{2}-P_{n_{1}+1}^{2}-\cdots-P_{n}^{2}=0$. Then, what we want is to understand how an arbitrary function on $C$ (of appropriate class) is expanded into joint eigenfunctions of $P_{1}, \ldots, P_{n}$.

We will find explicit joint eigendistributions for $P_{1}, \ldots, P_{n}$, and construct a (well-defined) transform, to be denoted by $\mathcal{F}_{C}$, by means of these eigenfunction. The transform $\mathcal{F}_{C}$ intertwines the multiplication by coordinate functions with the differential operators $P_{j}$. Moreover, we prove that we can normalize $\mathcal{F}_{C}$ such that it is involutive, i.e. $\mathcal{F}_{C}^{2}=\mathrm{id}$ and unitary. Thus, we establish its inversion formula and the Plancherel type theorem. It is noteworthy that the kernel function $K\left(x, x^{\prime}\right)$ of $\mathcal{F}_{C}$ involves singular distributions (e.g. normal derivatives of Dirac's delta function with respect to a hypersurface) but yet that the operator $\mathcal{F}_{C}$ is unitary in the general case where $n_{1}, n_{2}>1$ and $n_{1}+n_{2}>4$. In the case $n_{1}=1, n_{2}=1$ or $\left(n_{1}, n_{2}\right)=(2,2), \mathcal{F}_{C}$ reduces to the Hankel transform composed by a (singular) Radon transform.

The second motivation comes from representation theory of real reductive groups, in particular, from minimal representations.

Minimal representations are infinite dimensional unitary representations that are the 'closest' to the trivial one-dimensional representation. The Weil 
representation of the metaplectic group $M p(n, \mathbb{R})$, which plays a prominent role in the construction of theta series, is a classic example. Most minimal representations are isolated among the set of irreducible unitary representations, and cannot be built up from the exsisting induction techniques of representation theory.

A multitude of different models of minimal representations have been investigated recently by many people (see Sections 1.4 and 1.5). Each model known so far has its own advantages indeed but also has some disadvantages. For instance, the inner product of the Hilbert space is not explicit in some models, whereas the whole group action is not clear in some other models.

A challenge to surmount that 'disadvantage' may turn up as a natural problem in other areas of mathematics. In order to give its flavor, let us consider two geometric models of minimal representations of the indefinite orthogonal group $G=O\left(n_{1}+1, n_{2}+1\right)$ : One is in the solution space to the Yamabe equation (conformal model), and the other is in $L^{2}(C)$ (Schrödinger model).

In the conformal model, the whole group action is very clear, whereas the inner product is not. The problem of finding the explicit inner product was solved in the previous paper [47] as the theory of conserved quantities for ultra-hyperbolic equations, such as the energy for the wave equation.

In the Schrödinger model $L^{2}(C)$, the unitary structure is clear, whereas the whole group action is not. The understanding of the whole group action was a missing piece of [47]. This problem is reduced to finding the generalization of the Fourier-Hankel transform on the isotropic cone $C$, namely, the above mentioned operator $\mathcal{F}_{C}$. By finding an explicit formula of $\mathcal{F}_{C}$, we shall settle this problem. The role of $\mathcal{F}_{C}$ in our minimal representation is in parallel to that of the Euclidean Fourier transform $\mathcal{F}_{\mathbb{R}^{n}}$ in the Weil representation, summarized as below:

\begin{tabular}{l|cc} 
simple group & $\begin{array}{c}M p(n, \mathbb{R}) \\
(\text { type } C)\end{array}$ & $\begin{array}{c}O\left(n_{1}+1, n_{2}+1\right) \\
(\text { type } D)\end{array}$ \\
\hline Minimal representation & Weil representation & $\pi$ \\
\hline $\begin{array}{l}L^{2} \text {-model } \\
\text { (Schrödinger model) }\end{array}$ & $L^{2}\left(\mathbb{R}^{n}\right)$ & $L^{2}(C)$ \\
\hline unitary inversion & $e^{\frac{\sqrt{-1} n \pi}{4}} \mathcal{F}_{\mathbb{R}^{n}}$ & $\mathcal{F}_{C}$
\end{tabular}

The third motivation comes from special functions. We note that the 
isotropic cone $C$ is so small that the group $G=O\left(n_{1}+1, n_{2}+1\right)$ cannot act on $C$ continuously and non-trivially. This feature is reflected by the fact that the Gelfand-Kirillov dimension of the representation of $G$ on $L^{2}(C)$ attains its minimum amongst all infinite dimensional representations of $G$. Thus, the representation space $L^{2}(C)$ is extremely 'small' with respect to the group $G$. In turn, we could expect a very concrete theory of global analysis on $C$ by using abundant symmetries of the group $G$ or its Lie algebra.

It turns out that special functions in the Schrödinger model $L^{2}(C)$ arise in a somewhat different way from the well-known cases such as analysis on symmetric spaces (e.g. [31]) or its variants. For instance, the Casimir operator of $K$ acts on $L^{2}(C)$ as a fourth differential operator.

In this book, we encounter many classically known special functions (e.g. Bessel functions, Appell's hypergeometric functions, Meijer's $G$-functions, etc.). Special functions are a part of our method for the analysis of the minimal representation, and conversely, by decomposing the operator $\mathcal{F}_{C}$ we provide a representation theoretic proof of [inversion, Plancherel, ...] formulas of special functions including Meijer's $G$-functions.

Encouraged by a suggestion of R. Stanton, we have decided to write a considerably long introduction. What follows is divided into three parts according to the aforementioned three motivations and new perspectives. In Sections 1.1-1.3, we state key properties of the involutive unitary operator $\mathcal{F}_{C}$ on $L^{2}(C)$ from analytic perspectives, in comparison with the well-known case of the Euclidean Fourier transform $\mathcal{F}_{\mathbb{R}^{n}}$ on $L^{2}\left(\mathbb{R}^{n}\right)$. Sections 1.4 1.8 give representation theoretic perspectives, and we explain the role of $\mathcal{F}_{C}$ in the Schrödinger model of the minimal representation of the indefinite orthogonal group in comparison with the role of $\mathcal{F}_{\mathbb{R}^{n}}$ for the Weil representation. Thus, we compare $\mathcal{F}_{C}$ again with $\mathcal{F}_{\mathbb{R}^{n}}$, and correspondingly, the simple Lie algebra $\mathfrak{o}\left(n_{1}+1, n_{2}+1\right)$ with $\mathfrak{s p}(n, \mathbb{R})$. In Section 1.9, we give a flavor of the interactions of the analysis on the minimal representations with special functions.

\subsection{Differential operators on the isotropic cone}

Consider an indefinite quadratic form on $\mathbb{R}^{n}=\mathbb{R}^{n_{1}+n_{2}}$ :

$$
Q(x):=x_{1}^{2}+\cdots+x_{n_{1}}^{2}-x_{n_{1}+1}^{2}-\cdots-x_{n_{1}+n_{2}}^{2} .
$$

Throughout the Introduction, we assume $n_{1}, n_{2}>1$ and $n=n_{1}+n_{2}$ is an even integer greater than two. (From Chapter 2, we will use the following notation: $p=n_{1}-1, q=n_{2}-1$.) 
Associated to the quadratic form $Q$, we define the isotropic cone

$$
C:=\left\{x \in \mathbb{R}^{n} \backslash\{0\}: Q(x)=0\right\},
$$

and endow $C$ with the volume form $d \mu$ characterized by

$$
d Q \wedge d \mu=d x_{1} \cdots d x_{n} .
$$

Our object of study is the Hilbert space $L^{2}(C) \equiv L^{2}(C, d \mu)$ consisting of square integrable functions on $C$.

A differential operator $P$ on $\mathbb{R}^{n}$ is said to be tangential to the submanifold $C$ if $P$ satisfies

$$
\left.\psi_{1}\right|_{C}=\left.\left.\psi_{2}\right|_{C} \Rightarrow\left(P \psi_{1}\right)\right|_{C}=\left.\left(P \psi_{2}\right)\right|_{C}
$$

for any smooth functions $\psi_{1}, \psi_{2}$ defined in neighborhoods of $C$ in $\mathbb{R}^{n}$. Then, we can 'restrict' $P$ to $C$, and get a differential operator $\left.P\right|_{C}$ on $C$.

For instance, the following vector fields are tangential to $C$ :

$$
\begin{gathered}
E:=\sum_{i=1}^{n} x_{i} \frac{\partial}{\partial x_{i}} \quad(\text { the Euler operator }), \\
X_{i j}:=\epsilon_{i} \epsilon_{j} x_{i} \frac{\partial}{\partial x_{j}}-x_{j} \frac{\partial}{\partial x_{i}} \quad(1 \leq i<j \leq n),
\end{gathered}
$$

where we set $\epsilon_{j}=1$ or -1 according as $1 \leq j \leq n_{1}$ or $n_{1}+1 \leq j \leq n$. This is because the vector fields $E$ and $X_{i j}(1 \leq i<j \leq n)$ are obtained as the differential of the conformal linear transformation group

$$
C O(Q):=\left\{g \in G L(n, \mathbb{R}): Q(g x)=c Q(x)\left({ }^{\forall} x \in \mathbb{R}^{n}\right) \text { for some } c>0\right\},
$$

which preserves the isotropic cone $C$.

Let $\mathbb{R}\left[x, \frac{\partial}{\partial x}\right]$ be the $\mathbb{R}$-algebra of differential operators with polynomial coefficients (the Weyl algebra), namely, the non-commutative ring generated by the multiplication by $x_{1}, \ldots, x_{n}$ and the vector fields $\frac{\partial}{\partial x_{1}}, \ldots, \frac{\partial}{\partial x_{n}}$.

We denote by $\mathbb{R}\left[x, \frac{\partial}{\partial x}\right]^{C}$ the subalgebra consisting of operators that are tangential to $C$. The multiplication by coordinate functions $x_{k}$ clearly satisfies the condition (1.1.2). Thus, we have seen

$$
x_{k}, E, X_{i j} \in \mathbb{R}\left[x, \frac{\partial}{\partial x}\right]^{C} \quad(1 \leq k \leq n, 1 \leq i<j \leq n) .
$$

However, there exist yet other operators which are tangential to $C$, but are not generated by $x_{k}, E, X_{i j}$ in the Weyl algebra (see Remark 2.4.9). 
Among them are the fundamental differential operators of second order, to be denoted by $P_{1}, \ldots, P_{n}$, which are defined by

$$
P_{j}:=\epsilon_{j} x_{j} \square-(2 E+n-2) \frac{\partial}{\partial x_{j}} .
$$

Here, $\square$ is the Laplace-Beltrami operator associated to $Q$, namely,

$$
\square:=\sum_{j=1}^{n} \epsilon_{j} \frac{\partial^{2}}{\partial x_{j}^{2}} \equiv \frac{\partial^{2}}{\partial x_{1}^{2}}+\cdots+\frac{\partial^{2}}{\partial x_{n_{1}}^{2}}-\frac{\partial^{2}}{\partial x_{n_{1}+1}^{2}}-\cdots-\frac{\partial^{2}}{\partial x_{n}^{2}} .
$$

In the degenerate case $n_{1}=n_{2}=1$, our operators $P_{1}$ and $P_{2}$ take the following form: we set $y_{1}:=x_{1}+x_{2}, y_{2}:=x_{1}-x_{2}$,

$$
P_{1}+P_{2}=-4 y_{1} \frac{\partial^{2}}{\partial y_{1}^{2}}, \quad P_{1}-P_{2}=-4 y_{2} \frac{\partial^{2}}{\partial y_{2}^{2}}
$$

see Remark 2.4.10, In general, these operators $P_{1}, \ldots, P_{n}$ satisfy the following properties (see Theorem 2.4.1):

P1 $P_{i} P_{j}=P_{j} P_{i}$ for any $1 \leq i, j \leq n$.

P2 $P_{j} \in \mathbb{R}\left[x, \frac{\partial}{\partial x}\right]^{C}$ for any $1 \leq j \leq n$.

P3 The induced differential operators $\left.P_{j}\right|_{C}$ on $C_{0}^{\infty}(C)$ extend to self-adjoint operators on the Hilbert space $L^{2}(C)$.

$\left.\mathbf{P 4}\left(P_{1}^{2}+\cdots+P_{n_{1}}^{2}-P_{n_{1}+1}^{2}-\cdots-P_{n}^{2}\right)\right|_{C}=0$.

P5 The Lie algebra generated by $x_{i}, P_{i}(1 \leq i \leq n)$ contains the vector fields $E, X_{i j}(1 \leq i<j \leq n)$.

From now on, we simply write $P_{j}$ for $\left.P_{j}\right|_{C}$. Thus, we have commuting self-adjoint, second-order differential operators $P_{1}, \ldots, P_{n}$ on $L^{2}(C)$.

We are brought naturally to the following:

Problem 1.1.1. 1) Find joint eigenfunctions of the differential operators $P_{1}, \ldots, P_{n}$ on the isotropic cone $C$.

2) Given a function $f$ on $C$, find an explicit expansion formula of $f$ into joint eigenfunctions of $P_{1}, \ldots, P_{n}$. 


\section{2 'Fourier transform' $\mathcal{F}_{C}$ on the isotropic cone $C$}

In this book, we shall give a solution to Problem 1.1.1 by introducing a unitary operator $\mathcal{F}_{C}$ on $L^{2}(C)$.

To elucidate the operator $\mathcal{F}_{C}$, let us consider first much simpler operators

$$
p_{j}:=-\sqrt{-1} \frac{\partial}{\partial x_{j}} \quad(1 \leq j \leq n)
$$

in place of $P_{j}$. Then, $p_{1}, \ldots, p_{n}$ form a commuting family of differential operators which extend to self-adjoint operators on $L^{2}\left(\mathbb{R}^{n}\right)$. Analogously to Problem 1.1.1, consider the question of finding the explicit eigenfunction expansion for the operators $p_{1}, \ldots, p_{n}$. Then, as is well-known, this is done by using the (Euclidean) Fourier transform $\mathcal{F} \equiv \mathcal{F}_{\mathbb{R}^{n}}$ on $\mathbb{R}^{n}$. In what follows, we normalize $\mathcal{F}_{\mathbb{R}^{n}}$ as

$$
\mathcal{F}_{\mathbb{R}^{n}} u(\xi):=\frac{1}{(2 \pi)^{\frac{n}{2}}} \int_{\mathbb{R}^{n}} u(x) e^{\sqrt{-1}\langle x, \xi\rangle} d x,
$$

where $\langle x, \xi\rangle=\sum_{i=1}^{n} x_{i} \xi_{i}$ and $d x=d x_{1} \cdots d x_{n}$. We note that the signature of the power here is opposite from the usual convention. Obviously, the kernel

$$
k(x, \xi):=\frac{1}{(2 \pi)^{\frac{n}{2}}} e^{\sqrt{-1}\langle x, \xi\rangle}
$$

of the Fourier transform $\mathcal{F}_{\mathbb{R}^{n}}$ is real analytic on the direct product space $\mathbb{R}^{n} \times \mathbb{R}^{n}$.

We recall the following key properties of the Euclidean Fourier transform:

F1 $p_{j} k(x, \xi)=\xi_{j} k(x, \xi)$.

F2 $k(x, \xi)=k(\xi, x)$.

F3 $\mathcal{F}_{\mathbb{R}^{n}}\left(C_{0}^{\infty}\left(\mathbb{R}^{n}\right)\right) \subset C^{\infty}\left(\mathbb{R}^{n}\right) \cap L^{2}\left(\mathbb{R}^{n}\right)$.

F4 $\mathcal{F}_{\mathbb{R}^{n}}$ extends to a unitary operator on $L^{2}\left(\mathbb{R}^{n}\right)$.

F5 $\mathcal{F}_{\mathbb{R}^{n}} \circ x_{j}=p_{j} \circ \mathcal{F}_{\mathbb{R}^{n}}$,

$$
\mathcal{F}_{\mathbb{R}^{n}} \circ p_{j}=-\xi_{j} \circ \mathcal{F}_{\mathbb{R}^{n}} .
$$

F6 $\left(\mathcal{F}_{\mathbb{R}^{n}}^{2} u\right)(x)=u(-x), \mathcal{F}_{\mathbb{R}^{n}}^{4}=\mathrm{id}$.

F7 $\mathcal{F}_{\mathbb{R}^{n}} u(\xi)=\frac{1}{(2 \pi)^{\frac{n}{2}}} \int_{\mathbb{R}} e^{\sqrt{-1} t}(R u)(\xi, t) d t$. 
F8 $\mathcal{F}_{\mathbb{R}^{n}}$ gives an automorphism of each of the following topological vector spaces:

$$
\mathcal{S}\left(\mathbb{R}^{n}\right) \subset L^{2}\left(\mathbb{R}^{n}\right) \subset \mathcal{S}^{\prime}\left(\mathbb{R}^{n}\right)
$$

Here, F 7 gives the plane wave expansion of the Fourier transform by means of the Radon transform $R$ defined by

$$
R u(\xi, t):=\int_{\mathbb{R}^{n}} u(x) \delta(\langle x, \xi\rangle-t) d x .
$$

In $\mathbf{F} \mathbf{8}$, we denote by $\mathcal{S}\left(\mathbb{R}^{n}\right)$ the space of rapidly decreasing $C^{\infty}$-functions on $\mathbb{R}^{n}$ (the Schwartz space endowed with the Fréchet topology), and by $\mathcal{S}^{\prime}\left(\mathbb{R}^{n}\right)$ the dual space consisting of tempered distributions.

$\mathbf{F} 4$ is the Plancherel theorem, and F8 gives the Paley-Winer theorem for the Schwartz space $\mathcal{S}\left(\mathbb{R}^{n}\right)$ (and its dual $\mathcal{S}^{\prime}\left(\mathbb{R}^{n}\right)$ ). By $\mathbf{F} \mathbf{1}$ and $\mathbf{F} \mathbf{2}$, the inversion formula

$$
\begin{aligned}
f(x) & =\mathcal{F}_{\mathbb{R}^{n}} \circ \mathcal{F}_{\mathbb{R}^{n}}^{-1} f(x) \\
& =\int_{\mathbb{R}^{n}}\left(\mathcal{F}_{\mathbb{R}^{n}}^{-1} f\right)(\xi) k(x, \xi) d \xi
\end{aligned}
$$

gives an expansion of a function $f$ into joint eigenfunctions $k(x, \xi)$ of the commuting self-adjoint operators $p_{j}(1 \leq j \leq n)$.

Moreover, the property $\mathbf{F} \mathbf{5}$ characterizes the operator $\mathcal{F}_{\mathbb{R}^{n}}$ up to scalar. We pin down this algebraic statement in two ways as follows:

Proposition 1.2.1. Let $A$ be a continuous operator on $L^{2}\left(\mathbb{R}^{n}\right)$ satisfying the following identities:

$$
A \circ x_{j}=p_{j} \circ A, A \circ p_{j}=-x_{j} \circ A \quad(1 \leq j \leq n) .
$$

Then, $A$ is a scalar multiple of $\mathcal{F}_{\mathbb{R}^{n}}$.

Proposition 1.2.2. Let $A$ be a continuous operator on $L^{2}\left(\mathbb{R}^{n}\right)$ satisfying

$$
A \circ x_{i} x_{j}=p_{i} p_{j} \circ A, A \circ p_{i} p_{j}=x_{i} x_{j} \circ A \quad(1 \leq i, j \leq n) .
$$

Then, $A$ is of the form $A=a \mathcal{F}_{\mathbb{R}^{n}}+b \mathcal{F}_{\mathbb{R}^{n}}^{-1}$ for some $a, b \in \mathbb{C}$.

Here, (1.2.3) is obviously a weaker condition than (1.2.2).

We did not go into details about the domain of definition for (1.2.2) and $(1.2 .3)$ in the above propositions. The domain could be $D:=\{f \in$ 
$\left.L^{2}\left(\mathbb{R}^{n}\right): x_{j} f, p_{j} f \in L^{2}\left(\mathbb{R}^{n}\right)\left(1 \leq{ }^{\forall} j \leq n\right)\right\}$, on which we regard the identities $A x_{j} f=p_{j} A f$ those of distributions in the case of Proposition 1.2.1. Likewise for Proposition 1.2.2.

\section{Intertwining characterization of $\mathcal{F}_{C}$}

Back to the setting in Section 1.1, we consider the differential operator $P_{j}$ (of second order!). Then, it turns out that the intertwining relation between

$P_{j}$ and the multiplication by the coordinate function $x_{j}$ again characterizes our operator $\mathcal{F}_{C}$ up to scalar:

Theorem 1.2.3 (see Theorem 2.5.4). 1) There exists a unitary operator $\mathcal{F}_{C}$ on $L^{2}(C)$ satisfying the following relation:

$$
A \circ P_{j}=4 x_{j} \circ A, A \circ x_{j}=4 P_{j} \circ A \quad(1 \leq j \leq n) .
$$

2) Continuous operators $A$ on $L^{2}(C)$ satisfying (1.2.4) are unique up to scalar. In particular, any such operator $A$ is a scalar multiple of the unitary operator $\mathcal{F}_{C}$, and $A^{2}$ is a scalar multiple of the identity operator.

\section{Joint eigendistributions on $C$}

Next, we consider the system of differential equations on $C$ :

$$
P_{j} \psi=4 \xi_{j} \psi \quad(1 \leq j \leq n) .
$$

The coefficient 4 in the right-hand side is just for simplifying later notation.

We shall deal with solutions in an appropriate class of distributions on $C$ (the dual space $L^{2}(C)^{-\infty}$ of smooth vectors $L^{2}(C)^{\infty}$, see $(2.5 .9)$ ) and prove the following:

Theorem 1.2.4 (see Theorem 2.5.5). Fix $\xi=\left(\xi_{1}, \ldots, \xi_{n}\right) \in \mathbb{R}^{n} \backslash\{0\}$.

1) If $Q(\xi) \neq 0$, then any distribution $\psi$ on $C$ satisfying (1.2.5) is zero.

2) If $\xi \in C$, then the solution space of (1.2.5) in $L^{2}(C)^{-\infty}$ is one-dimensional.

The first statement is an immediate consequence of $\mathbf{P} \mathbf{4}$. By the explicit formula given in Theorem 1.3.1, we shall see that the unique solution in Theorem 1.2.4 (2) is not real analytic if $n_{1}, n_{2}>1$.

\section{Abstract properties of $\mathcal{F}_{C}$}

We will prove in this book that the distribution solution $\psi(x)$ in Theorem 1.2.4 (2) can be normalized depending on $\xi \in C$, which we denote by $K(x, \xi)$ for now, in such a way that the following key properties are fulfilled: 
K1 For each fixed $\xi \in C, K(\cdot, \xi)$ is a distribution solution on $C$ to (1.2.5).

K2 $K(x, \xi)=K(\xi, x)$ as a distribution on $C \times C$.

K3 We define

$$
\left(\mathcal{F}_{C} f\right)(\xi):=\int_{C} K(x, \xi) f(x) d \mu(x) \quad \text { for } f \in C_{0}^{\infty}(C) .
$$

Then, (1.2.6) is well-defined, and we have a linear map $\mathcal{F}_{C}: C_{0}^{\infty}(C) \rightarrow$ $C^{\infty}(C) \cap L^{2}(C)$.

K4 $\mathcal{F}_{C}$ extends to a unitary operator on $L^{2}(C)$.

K5 $\mathcal{F}_{C} \circ 4 x_{j}=P_{j} \circ \mathcal{F}_{C}$,

$\mathcal{F}_{C} \circ P_{j}=4 \xi_{j} \circ \mathcal{F}_{C}$.

K6 $\mathcal{F}_{C}^{2}=\mathrm{id}$.

K7 $\mathcal{F}_{C} u(\xi)=\int_{\mathbb{R}} \Psi(t) \mathcal{R} f(\xi, t) d t$

K8 $\mathcal{F}_{C}$ gives the automorphism of each of the following topological vector spaces:

$$
L^{2}(C)^{\infty} \subset L^{2}(C) \subset L^{2}(C)^{-\infty}
$$

These properties $\mathbf{K} \mathbf{1}-\mathbf{K} \mathbf{8}$ are stated in parallel to the Euclidean case F1$\mathbf{F} 8$. In K7, $\mathcal{R}$ is the (singular) Radon transform on the isotropic cone $C$ which will be defined in (1.2.7), and $\Psi(t)$ is a distribution on $\mathbb{R}$ which will be defined in Theorem 1.3.1. We note that the transform in K/7 by $\Psi(t)$ collapses to the Hankel if $n_{2}=1$.

In $\mathbf{K} \mathbf{8}$, we have the following inclusive relation

$$
C_{0}^{\infty}(C) \subset L^{2}(C)^{\infty} \subset L^{2}(C) \subset L^{2}(C)^{-\infty} \subset \mathcal{D}^{\prime}(C)
$$

as in the Euclidean case (see $\mathbf{F} \mathbf{8}$ ):

$$
C_{0}^{\infty}\left(\mathbb{R}^{n}\right) \subset \mathcal{S}\left(\mathbb{R}^{n}\right) \subset L^{2}\left(\mathbb{R}^{n}\right) \subset \mathcal{S}^{\prime}\left(\mathbb{R}^{n}\right) \subset \mathcal{D}^{\prime}\left(\mathbb{R}^{n}\right) .
$$

In summary, $\mathbf{K} \underline{4}$ is a Plancherel type theorem of $\mathcal{F}_{C}$ on $L^{2}(C), \mathbf{K} \underline{\mathbf{6}}$ gives its inversion formula, $\mathbf{K} \mathbf{7}$ expresses $\mathcal{F}_{C}$ by 'plane wave' decomposition, and $\mathbf{K} 8$ gives a Paley-Winer type theorem for the 'Schwartz space' $L^{2}(C)^{\infty}$.

The above formulation brings us naturally to the following program: 
Program 1.2.5. Develop a theory of 'Fourier analysis' on the isotropic cone $C$ by means of $\mathcal{F}_{C}$.

We expect that this program could be enhanced by a solid foundation and concrete formulas of the transform $\mathcal{F}_{C}$.

For this, the first step is to find explicit formulas for the (normalized) joint eigenfunctions $K(x, \xi)$. We prove that they are given by means of Bessel distributions (see Theorem 1.3.1). In particular, K2 follows readily from the formulas. The properties K1, K4, $\mathbf{4} \mathbf{5}$, and $\mathbf{K} \mathbf{6}$ will be proved in Theorem 2.5.2 based on a representation theoretic interpretation that $\mathcal{F}_{C}$ is the 'unitary inversion operator' on $L^{2}(C)$ for the minimal representation of the indefinite orthogonal group $O\left(n_{1}+1, n_{2}+1\right)$. By KE , we get the inversion formula just as $\mathcal{F}_{C}^{-1}=\mathcal{F}_{C}$, which gives an explicit solution to the problem of joint eigenfunction expansions (see Problem 1.1.1):

$$
f(x)=\int_{C}\left(\mathcal{F}_{C} f\right)(\xi) K(x, \xi) d \mu(\xi) .
$$

The kernel $K(x, \xi)$ is not locally integrable but is a distribution in general. To see the convergence of the right-hand side (1.2.6), we note that $K(x, \xi)$ depends only on $\langle x, \xi\rangle=\sum_{i=1}^{n} x_{i} \xi_{i}$ (see Theorem 1.3.1). This fact leads us to the factorization $\mathbf{K} \mathbf{7}$ through the (singular) Radon transform $\mathcal{R}$ on the isotropic cone $C$, which is defined by the integration over the intersection of $C$ with the hyperplane

$$
\left\{x \in \mathbb{R}^{n}:\langle x, \xi\rangle=t\right\} .
$$

For a quick summary of the transform $\mathcal{R}$ (see Chapter 5 for details), we identify a compactly supported smooth function $f$ on $C$ with a measure $f d \mu$. It is a tempered distribution on $\mathbb{R}^{n}(n>2)$. Then, the Radon transform $\mathcal{R}$ of $f d \mu$ is defined by

$$
\mathcal{R} f(\xi, t):=\int_{C} f(x) \delta(\langle x, \xi\rangle-t) d \mu(x)
$$

for $(\xi, t) \in\left(\mathbb{R}^{n} \backslash\{0\}\right) \times(\mathbb{R} \backslash\{0\})$. The point here is that the integration is taken over the isotropic cone. In other words, $\mathcal{R} f(\xi, t)$ is obtained by the integration over submanifolds which are generically of codimension two in $\mathbb{R}^{n}$. Consequently, $\mathcal{R} f(\xi, t)$ satisfies the ultra-hyperbolic differential equation of the $\xi$-variable:

$$
\left(\sum_{j=1}^{n_{1}} \frac{\partial^{2}}{\partial \xi_{j}^{2}}-\sum_{j=n_{1}+1}^{n} \frac{\partial^{2}}{\partial \xi_{j}^{2}}\right) \mathcal{R} f(\xi, t)=0 .
$$


Next, in order to see the regularity of $\mathcal{R} f(\xi, t)$ at $t=0$, we fix $\xi$. Then, the intersection of the isotropic cone $C$ with the hyperplane $\left\{x \in \mathbb{R}^{n}\right.$ : $\langle x, \xi\rangle=t\}$ forms a one parameter family of submanifolds of codimension two for $t \neq 0$, which have singularities at $t=0$. Accordingly, the Radon transform $\mathcal{R} f(\xi, t)$ is not of $C^{\infty}$ class at $t=0$ even for $f \in C_{0}^{\infty}(C)$. The regularity of $\mathcal{R} f(\xi, t)$ at $t=0$ is the principal object of the paper [54, where it is proved that $\mathcal{R} f(\xi, t)$ is $\left[\frac{n-5}{2}\right]$ times continuously differentiable at $t=0$. Here, $[x]$ denotes the greatest integer that does not exceed $x$. This regularity is exactly sufficient for what we need to prove that the singular integral (1.2.6) makes sense for $f \in C_{0}^{\infty}(C)$. See Section 5.2 for details.

The reverse direction, namely, the application of our results on $\mathcal{F}_{C}$ to the results on the singular Radon transform $\mathcal{R}$ includes:

Corollary 1.2.6. Any compactly supported smooth function $f \in C_{0}^{\infty}(C)$ can be recovered only from the restriction of the Radon transform $\mathcal{R} f(\xi, t)$ to $C \times \mathbb{R}$.

\section{Underlying algebraic structures}

The underlying algebraic structure of Propositions 1.2.1 and 1.2 .2 and Theorem 1.2 .3 will be revealed by the Lie algebras generated by the differential operators in each setting as follows:

In Proposition 1.2.1, the Lie algebra generated by $p_{i}, x_{i}(1 \leq i \leq n)$ is the Heisenberg Lie algebra.

In Proposition 1.2.2, the Lie algebra generated by $p_{i} p_{j}, x_{i} x_{j}(1 \leq i, j \leq$ $n)$ is the symplectic Lie algebra $\mathfrak{s p}(n, \mathbb{R})$.

In Theorem 1.2.3, the Lie algebra generated by $P_{i}, x_{i}\left(1 \leq i \leq n_{1}+n_{2}\right)$ is the indefinite orthogonal Lie algebra $\mathfrak{o}\left(n_{1}+1, n_{2}+1\right)$.

These actions of the Lie algebras lift to unitary representations of the corresponding Lie groups: On $L^{2}\left(\mathbb{R}^{n}\right)$, the Schrödinger representation of the Heisenberg group, and the Weil representation of the metaplectic group $M p(n, \mathbb{R})$, namely, a double cover of the symplectic group $S p(n, \mathbb{R})$ (see Section 1.4); on $L^{2}(C)$, the minimal representation of the indefinite orthogonal group $O\left(n_{1}+1, n_{2}+1\right)$ (see Section 1.5). In Sections 1.4-1.8, we shall discuss some perspectives from representation theory. Before entering representation theory, we continue an account from the viewpoints of analysis in the next section. 


\subsection{Kernel of $\mathcal{F}_{C}$ and Bessel distributions}

In this section, we give an explicit formula of the kernel distribution $K(x, \xi)$ of the transform $\mathcal{F}_{C}$ on $L^{2}(C)$, and observe the similarities to and differences from the kernel $k(x, \xi)=(2 \pi)^{-\frac{n}{2}} e^{\sqrt{-1}\langle x, \xi\rangle}$ of the Euclidean Fourier transform $\mathcal{F}_{\mathbb{R}^{n}}$ on $L^{2}\left(\mathbb{R}^{n}\right)$.

Here is the highlight of this book.

Theorem 1.3.1 (see Theorem 5.1.1). Suppose $n=n_{1}+n_{2}$ is even, $>2$. The unique unitary operator $\mathcal{F}_{C}$ on $L^{2}(C)$ in Theorem 1.2.3 is given by the distribution kernel $K(x, \xi):=\Psi(\langle x, \xi\rangle)$, where

$$
\begin{aligned}
& \Psi(t)=2(-1)^{\frac{n_{1}\left(n_{1}-1\right)}{2}} \pi^{-\frac{n-2}{2}} \\
& \times \begin{cases}\Phi_{\frac{n-4}{2}}^{+}(t) & \text { if } \min \left(n_{1}, n_{2}\right)=1, \\
\Psi_{\frac{n-4}{2}}^{+}(t) & \text { if } n_{1}, n_{2}>1 \text { are both odd, } \\
\Psi_{\frac{n-4}{2}}(t) & \text { if } n_{1}, n_{2}>1 \text { are both even. }\end{cases}
\end{aligned}
$$

As for the normalization of a scalar constant in the above theorem, we note that the intertwining property (1.2.4) determines $K(x, \xi)$ up to scalar, and moreover $\mathbf{K} \mathbf{6}$ determines $K(x, \xi)$ up to signature. The signature is taken to be compatible with the action on the Schrödinger model of the minimal representation of $O\left(n_{1}+1, n_{2}+1\right)$ which will be discussed in Section 1.8.

In Theorem 1.3.1, $\langle$,$\rangle denotes the standard (positive definite) inner$ product on $\mathbb{R}^{n}$. $\Phi_{m}^{+}(t), \Psi_{m}^{+}(t)$ and $\Psi_{m}(t)$ are tempered distributions on $\mathbb{R}$, defined below in (1.3.1), (1.3.2), and (1.3.3), respectively.

\section{Bessel distributions}

Let $J_{\nu}(x), Y_{\nu}(x)$ and $K_{\nu}(z)$ be the (modified) Bessel functions (see Appendix 7.2 ). We use the following notational convention:

$$
f\left(t_{+}\right):=\left\{\begin{array}{ll}
f(t) & (t>0) \\
0 & (t \leq 0),
\end{array} \quad f\left(t_{-}\right):= \begin{cases}0 & (t \geq 0) \\
f(|t|) & (t<0),\end{cases}\right.
$$

for a function (or a 'generalized function') $f(t)$ on $\mathbb{R}$. Then, $\Phi_{m}^{+}, \Psi_{m}^{+}$, and $\Psi_{m}$ in Theorem 1.3.1 are the distributions on $\mathbb{R}$ given by

$$
\begin{aligned}
& \Phi_{m}^{+}(t):=(2 t)_{+}^{-\frac{m}{2}} J_{m}\left(2 \sqrt{2 t_{+}}\right), \\
& \Psi_{m}^{+}(t):=(2 t)_{+}^{-\frac{m}{2}} J_{m}\left(2 \sqrt{2 t_{+}}\right)-\sum_{k=1}^{m} \frac{(-1)^{k-1}}{2^{k}(m-k) !} \delta^{(k-1)}(t), \\
& \Psi_{m}(t):=(2 t)_{+}^{-\frac{m}{2}} Y_{m}\left(2 \sqrt{2 t_{+}}\right)+\frac{2(-1)^{m+1}}{\pi}(2 t)_{-}^{-\frac{m}{2}} K_{m}\left(2 \sqrt{2 t_{-}}\right) .
\end{aligned}
$$


Here, $\left(2 t_{+}\right)^{-\frac{m}{2}} J_{m}\left(2 \sqrt{2 t_{+}}\right)$makes sense as a locally integrable function on $\mathbb{R}$. On the other hand, $(2 t)_{+}^{-\frac{m}{2}} Y_{m}\left(2 \sqrt{2 t_{+}}\right)$and $(2 t)_{-}^{-\frac{m}{2}} K_{m}\left(2 \sqrt{2 t_{-}}\right)$are defined as regularized distributions, and $\Psi_{m}(t)$ have the following singularity:

$$
\Psi_{m}(t)=(\text { locally integrable function })+\frac{-1}{\pi} \sum_{k=1}^{m} \frac{t^{-k}(k-1) !}{2^{k}(m-k) !} .
$$

We shall say that $\Phi_{m}^{+}, \Psi_{m}^{+}$, and $\Psi_{m}$ are Bessel distributions.

\section{Integral expressions and differential equations}

The Bessel distributions $\Phi_{m}^{+}(t), \Psi_{m}^{+}(t)$, and $\Psi_{m}^{+}(t)$ are real analytic on $\mathbb{R} \backslash\{0\}$, and satisfy the following differential equation:

$$
t \frac{d^{2} \Psi}{d t^{2}}+(m+1) \frac{d \Psi}{d t}+2 \Psi=0
$$

or equivalently,

$$
\left(\theta^{2}+m \theta+2 t\right) \Psi=0,
$$

where $\theta:=t \frac{d}{d t}$. Furthermore, all of the three solutions satisfy the following asymptotic behavior

$$
\Psi(t)=O\left(t^{-\frac{2 m+1}{4}}\right) \quad \text { as } t \rightarrow+\infty .
$$

In this book, we adopt an alternative definition of $\Phi_{m}^{+}, \Psi_{m}^{+}$and $\Psi_{m}$ in Section 6.2 by means of the Mellin-Barnes type integral for distributions. The expressions (1.3.1)-(1.3.3) will be explained there. Another (slightly different) expression of $\Phi_{m}^{+}, \Psi_{m}^{+}$, and $\Psi_{m}$ by means of 'normalized' Bessel functions $\widetilde{J}_{m}, \widetilde{K}_{m}$, and $\widetilde{Y}_{m}$ is given in Remark 6.2 .3 .

Chapter 6 is devoted to these Bessel distributions. We shall discuss their integral formulas and differential equations.

Support of the kernel $K(x, \xi)$

Unlike the kernel $k(x, \xi)=(2 \pi)^{-\frac{n}{2}} e^{\sqrt{-1}\langle x, \xi\rangle}$ for the Euclidean Fourier transform $\mathcal{F}_{\mathbb{R}^{n}}$, our formula in Theorem 1.3.1 shows that the support of the kernel $K(x, \xi)$ for $\mathcal{F}_{C}$ differs according to the signature $\left(n_{1}, n_{2}\right)$.

To see this, we set the 'half' space of the direct product manifold $C \times C$ by

$$
(C \times C)_{+}:=\{(x, \xi) \in C \times C:\langle x, \xi\rangle \geq 0\} .
$$

Then, we have the following mysterious phenomenon: 
Corollary 1.3.2. The kernel $K(x, \xi)$ of the unitary operator $\mathcal{F}_{C}$ on $L^{2}(C)$ satisfies

$$
\operatorname{supp} K(x, \xi)= \begin{cases}(C \times C)_{+} & \text {if } n_{1}, n_{2} \text { both odd } \\ C \times C & \text { if } n_{1}, n_{2} \text { both even } .\end{cases}
$$

In particular,

$$
\operatorname{supp} K(x, \xi) \varsubsetneqq C \times C \text { if } n_{1}, n_{2} \text { both odd. }
$$

If $n_{1}=1$ or $n_{2}=1$, the isotropic cone $C$ is the light cone in the relativistic cosmology, which splits into two connected components, namely, the forward light cone $C_{+}$and backward light cone $C_{-}$, and correspondingly, we have a direct sum decomposition as Hilbert spaces:

$$
L^{2}(C)=L^{2}\left(C_{+}\right) \oplus L^{2}\left(C_{-}\right) .
$$

Then, $\mathcal{F}_{C}$ leaves $L^{2}\left(C_{+}\right)$and $L^{2}\left(C_{-}\right)$invariant, respectively (see [44, 46]). This gives a geometric explanation of Corollary 1.3 .2 because

$$
(C \times C)_{+}=\left(C_{+} \times C_{+}\right) \cup\left(C_{-} \times C_{-}\right)
$$

in this case. On the other hand, if $n_{1}, n_{2}>1$, then $C$ is connected and we do not have a natural decomposition of $(C \times C)_{+}$like (1.3.5). Moreover, the representation $\pi$ of the indefinite orthogonal group $O\left(n_{1}+1, n_{2}+1\right)$ on $L^{2}(C)$ (discussed later) stays irreducible when restricted to the identity component $S O_{0}\left(n_{1}+1, n_{2}+1\right)$. Nevertheless, the support of $K(x, \xi)$ is half the space of $C \times C$ when both $n_{1}$ and $n_{2}$ are odd integers greater than one. We do not see its intrinsic reason.

\section{Singularities of the kernel $K(x, \xi)$}

Another distinguishing feature of our kernel $K(x, \xi)$ for $\mathcal{F}_{C}$ is that it is not real analytic on $C \times C$, whereas the kernel $k(x, \xi)=(2 \pi)^{-\frac{n}{2}} e^{\sqrt{-1}\langle x, \xi\rangle}$ for the Euclidean Fourier transform $\mathcal{F}_{\mathbb{R}^{n}}$ is obviously real analytic on $\mathbb{R}^{n} \times \mathbb{R}^{n}$.

Among the three Bessel distributions introduced in (1.3.1)-(1.3.3), $\Phi_{m}^{+}(t)$ is a locally integrable function on $\mathbb{R}$, whereas $\Psi_{m}^{+}(t)$ and $\Psi_{m}(t)(m \geq 1)$ are not. The singular part of the distribution $\Psi_{m}^{+}(t)$ is given as a linear combination of the Dirac delta function $\delta(t)$ and its $l$ th derivative $\delta^{(l)}(t)$ $(l=1,2, \ldots, m-1)$. The singular part of the distribution $\Psi_{m}(t)$ is given by a linear combination of the distribution $t^{-k}(k=1,2, \ldots, m)$ (see Theorem 6.2.1).

Some readers might wonder why the kernel function of a unitary operator involves such singularities. So, let us examine to which extent the regularity of the kernel $K(x, \xi)$ is required from the general theory of functional analysis. 
By the Schwartz kernel theorem, any continuous operator $T: L^{2}(C) \rightarrow$ $L^{2}(C)$ is expressed as

$$
(T f)(\xi)=\int_{C} K(x, \xi) f(x) d \mu(x)
$$

by some distribution kernel $K(x, \xi) \in \mathcal{D}^{\prime}(C \times C)$. Here, we regard $K(x, \xi)$ as a generalized function by using the measure $d \mu$ on $C$ (see [25]).

If $T$ is a Hilbert-Schmidt operator, then $K \in L^{2}(C \times C)$. If $T$ is the identity operator, then $K$ is Dirac's delta function $\delta(x-\xi)$. In general, the continuity of $T$ forces any such $K$ to be at most $(\operatorname{dim} C+2)$ times derivatives of a locally integrable function on $C \times C$ (see [70, pp. 296-299] for the argument using the Sobolev space theory in the compact torus case).

\section{'Laurent series expansions' of Bessel distributions}

We end this section with an interesting observation on 'Laurent series expansions' of Bessel distributions $\Phi_{m}^{+}(t)$ and $\Psi_{m}^{+}(t)$ :

$$
\begin{aligned}
\Phi_{m}^{+}(t) & =\sum_{j=0}^{\infty} \frac{(-1)^{j} 2^{j} t_{+}^{j}}{\Gamma(m+j+1) \Gamma(j+1)} \\
\Psi_{m}^{+}(t) & =\left(\sum_{j=0}^{\infty}+\sum_{j=-\infty}^{-1}\right) \frac{(-1)^{j} 2^{j} t_{+}^{j}}{\Gamma(m+j+1) \Gamma(j+1)} .
\end{aligned}
$$

We note that $\Phi_{m}^{+}$arises as the kernel for $\mathcal{F}_{C}$ when $\left(n_{1}, n_{2}\right)=(2 m+3,1)$, and that $\Psi_{m}^{+}$arises when $n_{1}, n_{2}, \geq 3$ are both odd and $n_{1}+n_{2}=2 m+4$. The first formula (1.3.6) is a usual Taylor expansion. But the second formula (1.3.7) involves negative terms, for which we need a justification. For this, we think of $t_{+}^{\lambda}$ as a distribution meromorphically dependent on $\lambda$, and then we get

$$
\left.\frac{t_{+}^{\lambda}}{\Gamma(1+\lambda)}\right|_{\lambda=-k}=\delta^{(k-1)}(t)
$$

by (7.1.1). Therefore, we have

$$
\left.\frac{(-1)^{\lambda} 2^{\lambda} t_{+}^{\lambda}}{\Gamma(m+\lambda+1) \Gamma(1+\lambda)}\right|_{\lambda=-k}= \begin{cases}\frac{(-1)^{k}}{2^{k}(m-k) !} \delta^{(k-1)}(t) & (1 \leq k \leq m), \\ 0 & (m+1 \leq k) .\end{cases}
$$

In this sense, the series (1.3.7) contains only finitely many negative terms, and is equal to (1.3.2). 


\subsection{Perspectives from representation theory - find- ing smallest objects}

The philosophy of analysis and synthesis asks for the understanding of the smallest objects and of how things are built from these objects.

For a Hilbert space $\mathcal{H}$ over $\mathbb{C}$, we denote by $U(\mathcal{H})$ the group consisting of unitary operators on $\mathcal{H}$. By a unitary representation on a Hilbert space $\mathcal{H}$ of a topological group $G$, we mean a group homomorphism

$$
\pi: G \rightarrow U(\mathcal{H})
$$

such that $G \times \mathcal{H} \rightarrow \mathcal{H},(g, v) \mapsto \pi(g) v$ is continuous.

The 'smallest objects' of unitary representations are irreducible unitary representations. By a theorem of Mautner and Teleman, any unitary representation of a locally compact group $G$ (e.g. a Lie group) can be decomposed into the direct integral of irreducible unitary representations of $G$, see [78]. The classification of irreducible unitary representations of Lie groups has been a long standing unsolved problem since 1940s, originally arising from quantum mechanics.

The 'smallest objects' of Lie groups consist of simple Lie groups such as $S L(n, \mathbb{R}), O(p, q)$, and $S p(n, \mathbb{R})$, and one-dimensional abelian Lie groups such as $\mathbb{R}$ and $S^{1}$. Loosely speaking, a theorem of Duflo [11] asserts that all irreducible unitary representations of general (real algebraic) Lie groups are built up from those of simple Lie groups.

However, irreducible unitary representations of simple Lie groups are not fully understood despite huge efforts for many decades and also significant results. Among them, powerful algebraic machinery including the theory of cohomological induction has been largely developed in 1980s by Zuckerman, Vogan, Wallach and others [77, 78. As a result, the problem of classifying irreducible unitary representations has been focused on those representations of simple Lie groups that cannot be 'induced up' from other representations. Such representations may be regarded as 'atoms' of unitary representations of Lie groups, and they are still mysterious creatures. See Vogan [77, for example, for a discussion on how to understand them as the theory of 'unipotent representations' of reductive Lie groups.

'Minimal representations' are the simplest, infinite dimensional 'unipotent representations'. There has been active study on minimal representations, mostly by algebraic methods since 1990s (see Gan and Savin [21] and J.-S. Li [52 for surveys both in the real and in the $p$-adic fields). In contrast to these existing algebraic approaches, new geometric analysis of 
the minimal representation of the simple Lie group $O(p, q)$ is a motif of this book. We will discuss minimal representations in the next section followed by Sections $1.6-1.8$ in more details.

\subsection{Minimal representations of simple Lie groups}

To formulate the 'smallness' of an irreducible representation $\pi$ of a simple Lie group $G$, algebraic representation theory usually appeals to the 'largeness' of the annihilator $\operatorname{Ann}(\pi)$ in $U\left(\mathfrak{g}_{\mathbb{C}}\right)$ of the differential representation $d \pi$. Here, $U\left(\mathfrak{g}_{\mathbb{C}}\right)$ is the universal enveloping algebra of the complexified Lie algebra $\mathfrak{g}_{\mathbb{C}}=\mathfrak{g} \otimes_{\mathbb{R}} \mathbb{C}$. We recall:

Definition 1.5.1. An irreducible unitary representation $\pi$ of a simple Lie group $G$ is a minimal representation if the annihilator $\operatorname{Ann}(\pi)$ is equal to the Joseph ideal [20, 37] of $U\left(\mathfrak{g}_{\mathbb{C}}\right)$.

The Joseph ideal $\operatorname{Ann}(\pi)$ is a completely prime ideal whose associated variety $\mathcal{V}(\operatorname{Ann} \pi)$ is the closure of $\mathcal{O}_{\min }^{\mathbb{C}}$. Here, $\mathcal{O}_{\text {min }}^{\mathbb{C}}$ is the complex minimal nilpotent orbit in $\mathfrak{g}_{\mathbb{C}}^{*}$ for the coadjoint representation. Therefore, the Gelfand-Kirillov dimension of $\pi$, to be denoted by $\operatorname{DIM} \pi$, satisfies

$$
\operatorname{DIM} \pi=\frac{1}{2} \operatorname{dim}_{\mathbb{C}} \mathcal{O}_{\text {min }}^{\mathbb{C}},
$$

and in particular, $\pi$ has the smallest possible Gelfand-Kirillov dimension.

The Weil representation $\varpi$ of the metaplectic group $M p(n, \mathbb{R})$ is a classic example of minimal representations (to be precise, the Weil representation decomposes into a direct sum of two irreducible representations, both of which are minimal representations), and DIM $\varpi=n$.

The study of minimal representations of other reductive groups is relatively new, and it is only in the last two decades that various models of minimal representations have been proposed and studied extensively (see [6, 17, 13, 17, 21, 27, 39, 40, 46, 47, 48, 49, 50, 52, 63, 73, 80] for instance).

We note that not every reductive Lie group admits minimal representations. For instance, the minimal nilpotent orbit $\mathcal{O}_{\min }^{\mathbb{C}}$ of $S O(m, \mathbb{C})$ has the dimension $2(m-3)$, and it was found by Howe and Vogan (see [76]) that there exists no representation of any covering group of $S O_{0}(p, q)$ (or its finite extension) whose Gelfand-Kirillov dimension equals $p+q-3$ if $p+q$ is odd and $p, q>3$. This is the primary reason that we dealt with the case $n=n_{1}+n_{2}$ is even in Sections 1.1-1.3. Here, $p=n_{1}+1$ and $q=n_{2}+1$. (It is another story that there exists an 'infinitesimally unitary' and 'minimal' representation of the Lie algebra $\mathfrak{s o}(p, q)$ for $p+q$ odd, see [49].) 
In accordance with the philosophy of the orbit method advocated by A. Kirillov, B. Kostant, and M. Duflo, irreducible unitary representations of Lie groups $G$ are supposed to be attached to coadjoint orbits in $\mathfrak{g}^{*}$. This works perfectly for simply-connected nilpotent Lie groups. However, for simple Lie groups, the orbit method does not work very well, though the orbit method still gives an approximation of the unitary dual. In particular, it is not known how to attach unitary representations to nilpotent orbits of simple Lie groups.

The reverse direction is easier. As we have seen at the beginning of this section, we can attach nilpotent orbits to admissible representations $\pi$ (in particular, to irreducible unitary representations):

$$
\pi \rightsquigarrow \operatorname{Ann}(\pi) \rightsquigarrow \mathcal{V}(\operatorname{Ann}(\pi)) .
$$

There are also several ways to attach (a union of) real nilpotent orbits in $\mathfrak{g}^{*}$ to admissible representations $\pi$ such as

$\mathfrak{g}^{*} \supset \mathrm{WF}_{\mathrm{N}}(\pi)$ : wave front set by Howe, 34

$\mathfrak{g}^{*} \supset \mathrm{WF}(\pi)$ : asymptotic support of the character Trace $\pi$, 4]

$\mathfrak{p}_{\mathbb{C}}^{*} \supset \operatorname{Ass}(\pi)$ : associated variety of the underlying $\left(\mathfrak{g}_{\mathbb{C}}, K\right)$-module of $\pi$, [75]

Here, $\mathfrak{g}=\mathfrak{k}+\mathfrak{p}$ is a Cartan decomposition and $\mathfrak{g}_{\mathbb{C}}=\mathfrak{k}_{\mathbb{C}}+\mathfrak{p}_{\mathbb{C}}$ is its complexification. $\operatorname{WF}_{\mathrm{N}}(\pi)$ and $\mathrm{WF}(\pi)$ are a union of nilpotent orbits of $G$ in $\mathfrak{g}^{*}$, and $\operatorname{Ass}(\pi)$ is a union of nilpotent orbits of $K_{\mathbb{C}}$ on $\mathfrak{p}_{\mathbb{C}}^{*}$. It was conjectured by Barbash and Vogan [4], and proved by Schmid and Vilonen [64] that $\mathrm{WF}(\pi)$ corresponds to $\operatorname{Ass}(\pi)$ via the Kostant-Sekiguchi correspondence. For a minimal representation $\pi$, these invariants are contained in the closure of the intersection $\mathfrak{g}^{*} \cap \mathcal{O}_{\min }^{\mathbb{C}}, \mathfrak{p}_{\mathbb{C}}^{*} \cap \mathcal{O}_{\text {min }}^{\mathbb{C}}$, respectively. Here, $\mathcal{O}_{\text {min }}^{\mathbb{C}}$ is the complex minimal nilpotent orbit in $\mathfrak{g}_{\mathbb{C}}^{*}$, and we identify $\mathfrak{g}_{\mathbb{C}}$ with $\mathfrak{g}_{\mathbb{C}}^{*}$ by the Killing form.

Conversely, it is much more difficult to construct irreducible unitary representations from nilpotent orbits in general. If the complex minimal nilpotent orbit $\mathcal{O}_{\text {min }}^{\mathbb{C}}$ has a non-empty intersection with $\mathfrak{g}^{*}$, then $\mathcal{O}_{\text {min }}^{\mathbb{C}} \cap \mathfrak{g}^{*}$ consists of equi-dimensional $\operatorname{Ad}^{*}(G)$-orbits, namely, minimal nilpotent orbits in $\mathfrak{g}^{*}$. An optimistic picture is that minimal representations are attached to such orbits, however, this is false in general (see 72 for the $\mathfrak{s l}(3, \mathbb{R})$ case). In this direction Brylinski and Kostant [7] constructed minimal representations from $\operatorname{Ass}(\pi)$ on the $\left(\mathfrak{g}_{\mathbb{C}}, K\right)$-module level, but both the Hilbert structure and the whole group action are not given globally, depending on each $K$-type.

In this book, we deal with $G=O\left(n_{1}+1, n_{2}+1\right)\left(n_{1}, n_{2}>1, n_{1}+n_{2}\right.$ even $>4$ ), where $\mathcal{O}_{\text {min }}:=\mathcal{O}_{\text {min }}^{\mathbb{C}} \cap \mathfrak{g}^{*}$ is a single $G$-orbit, namely, a minimal nilpotent orbit. Our minimal representation is realized on the concrete 
Hilbert space $L^{2}(C)$. On the other hand, the isotropic cone $C$ becomes a Lagrangian submanifold of $\mathcal{O}_{\text {min }}$ which is endowed with the natural symplectic structure. In this sense, our Schrödinger model $L^{2}(C)$ may be viewed as a geometric quantization of the real minimal coadjoint orbit $\mathcal{O}_{\min }$. Our main theorem enables us to give the whole group $G$-action on $L^{2}(C)$ explicitly and independently of $K$-types.

\subsection{Schrödinger model for the Weil representation}

In Section 1.7, we shall discuss the minimal representation of $G=O\left(n_{1}+\right.$ $\left.1, n_{2}+1\right)$, which is a simple group of type $D$ if $n_{1}+n_{2}$ is even, $>2$. In this section, we recall the best understood minimal representation of a simple group of type $C$, that is, the Segal-Shale-Weil representation $\varpi$, simply, the Weil representation, or sometimes referred to as the oscillator representation, or harmonic representation, of the metaplectic group $M p(n, \mathbb{R})$. Here, $M p(n, \mathbb{R})$ is the twofold cover of the real symplectic group $S p(n, \mathbb{R})$. Let $\xi_{0}$ denote the (unique) non-trivial element in the kernel of the homomorphism $M p(n, \mathbb{R}) \rightarrow S p(n, \mathbb{R})$. That is, we have an exact sequence of Lie groups:

$$
1 \rightarrow\left\{e, \xi_{0}\right\} \rightarrow M p(n, \mathbb{R}) \rightarrow S p(n, \mathbb{R}) \rightarrow 1 .
$$

We will set $G^{\prime}:=M p(n, \mathbb{R})$.

Among various realizations of the Weil representation (see [52, §3] for a brief survey), the Schrödinger model gives a realization of the Weil representation $\varpi$ on the Hilbert space $L^{2}\left(\mathbb{R}^{n}\right)$. Since our model $\left(\pi, L^{2}(C)\right)$ of the minimal representation of $G$ has a strong resemblance to $\left(\varpi, L^{2}\left(\mathbb{R}^{n}\right)\right)$ of $G^{\prime}$, we list some important aspects of the Schrödinger model of $\varpi$ (see e.g. [18, 22, 35, 38]):

C1 The representation is realized on a very concrete Hilbert space, that is, $L^{2}\left(\mathbb{R}^{n}\right)$.

C2 The restriction of $\varpi$ to the Siegel parabolic subgroup $P_{\text {Siegel }}$ is still irreducible. The restriction $\varpi_{P_{\text {Siegel }}}$ has a relatively simple form (translations and multiplications by unitary characters).

C3 The infinitesimal action $d \varpi$ of the Lie algebra $\mathfrak{s p}(n, \mathbb{R})$ is given by differential operators of at most second order.

C4 There is a distinguished element $w_{0}^{\prime}$ of $G^{\prime}$ that sends $P_{\text {Siegel }}$ to the opposite parabolic subgroup. The corresponding unitary operator $\varpi\left(w_{0}^{\prime}\right)$ 
on $L^{2}\left(\mathbb{R}^{n}\right)$ is equal to $e^{\frac{\sqrt{-1} n \pi}{4}} \mathcal{F}_{\mathbb{R}^{n}}$. Correspondingly to the fact that $\left(w_{0}^{\prime}\right)^{4}=\xi_{0}$ and $\varpi\left(\xi_{0}\right)=-\mathrm{id}$, the Fourier transform $\mathcal{F}_{\mathbb{R}^{n}}$ is of order four.

We write $S p(n, \mathbb{R})$ in the matrix form as

$$
S p(n, \mathbb{R})=\left\{g \in G L(2 n, \mathbb{R}):{ }^{t} g J_{n} g=J_{n}\right\},
$$

where $J_{n}=\left(\begin{array}{cc}0 & -I_{n} \\ I_{n} & 0\end{array}\right)$. Then, down to $S p(n, \mathbb{R}) \simeq M p(n, \mathbb{R}) \bmod \left\{e, \xi_{0}\right\}$, we may take $P_{\text {Siegel }}$ and $w_{0}^{\prime}$ as

$$
\begin{aligned}
P_{\text {Siegel }} \bmod \left\{e, \xi_{0}\right\} & \simeq\left\{\left(\begin{array}{cc}
A & B \\
0 & { }^{t} A^{-1}
\end{array}\right): A \in G L(n, \mathbb{R}), A^{t} B=B^{t} A\right\}, \\
w_{0}^{\prime} \bmod \left\{e, \xi_{0}\right\} & =J_{n} .
\end{aligned}
$$

Since $G^{\prime}$ is generated by $P_{\text {Siegel }}$ and $w_{0}^{\prime}, \mathbf{C} \mathbf{2}$ and $\mathbf{C} 4$ determine the action of $G^{\prime}$ on $L^{2}\left(\mathbb{R}^{n}\right)$ (see [59] for an explicit formula of the whole group $G^{\prime}$-action on $L^{2}\left(\mathbb{R}^{n}\right)$ ). As for $\mathbf{C} \underline{\mathbf{3}}$, if $X \notin \mathfrak{p}_{\text {Siegel }}$ (the Lie algebra of $\left.P_{\text {Siegel }}\right)$, then $d \varpi(X)$ contains a differential operator of second order, and is not given by a vector field. This reflects the fact that $G^{\prime}$ acts only on $L^{2}\left(\mathbb{R}^{n}\right)$, and not on $\mathbb{R}^{n}$.

In various places of this book (usually, as Remarks), we will compare our results with the corresponding results for the Weil representation. We will see that the case $n_{1}=1$ or $n_{2}=1$ in our setting $G=O\left(n_{1}+1, n_{2}+1\right)$ has similar features to the case of the Weil representation in both analytic and representation theoretic aspects, and that the general case $n_{1}, n_{2}>1$ often provides new analytic features.

\subsection{Schrödinger model for the minimal represen- tation of $O(p, q)$}

In this section, we consider the indefinite orthogonal group

$$
G:=O(p, q)=\left\{g \in G L(p+q, \mathbb{R}):{ }^{t} g I_{p, q} g=I_{p, q}\right\},
$$

where $I_{p, q}:=\left(\begin{array}{cc}I_{p} & 0 \\ 0 & -I_{q}\end{array}\right), p, q \geq 2$ and $p+q$ is an even integer greater than four. Then, there exists a distinguished irreducible unitary representation $\pi$ of $G$ with the following properties:

M1 $\pi$ is still irreducible when restricted to the identity component $S O_{0}(p, q)$ of $G$ if and only if $p, q>2$. 
M2 If $p=2$ or $q=2$, then $\pi$ is a direct sum of an irreducible unitary highest weight representation and a lowest weight representation.

M3 $\pi$ is spherical (i.e. has a non-trivial vector fixed by $K=O(p) \times O(q)$ ) if and only if $p=q$.

M4 $\pi$ is a minimal representation in the sense of Definition 1.5.1 if $p+q>6$ (see [6, 50]).

M5 $\operatorname{WF}(\pi)=\mathcal{O}_{\text {min }}=\left(\mathcal{O}_{\text {min }}^{\mathbb{C}} \cap \mathfrak{g}^{*}\right), \operatorname{Ass}(\pi)=\mathcal{O}_{\text {min }}^{\mathbb{C}} \cap \mathfrak{p}_{\mathbb{C}}^{*}$, and $\operatorname{DIM}(\pi)=$ $p+q-3$, (see [47).

The above properties M2 and M3 show that our minimal representation $\pi$ is neither a spherical representation nor a highest weight representation in the generic case where $p \neq q, p, q>2$, and $p+q>6$.

Various realizations of the minimal representation $\pi$ have been proposed so far by a number of people. For example, Kazhdan in [39], and Kostant in [50] for $p=q=4$, and Binegar and Zierau [6] for general $p, q \geq 2$, constructed $\pi$ as a subrepresentation of a maximally degenerate principal series representation (see also Howe and Tan [36] for a full discussion on its composition series); Zhu and Huang [80] constructed $\pi$ as the theta correspondence of the trivial one-dimensional representation of $S L(2, \mathbb{R})$ for the reductive dual pair $O(p, q) \cdot S L(2, \mathbb{R}) \subset S p(p+q, \mathbb{R})$, see also [61]; and Kobayashi and Ørsted [47] constructed $\pi$ as the solution to the Yamabe equation (conformal model); in 49] the Schrödinger model (an $L^{2}$-model) of $\pi$. Yet another construction has been proposed in Brylinski and Kostant [7], Gross and Wallach [27, and Torasso [73].

Among various realizations of the minimal representations, our concern is with the Schrödinger model realized on $L^{2}(C)$. Here, $C$ is the isotropic cone in $\mathbb{R}^{n_{1}+n_{2}}=\mathbb{R}^{n}$ discussed in Sections 1.1-1.3, and the relation between the above parameters $p$ and $q$ is given by

$$
p=n_{1}+1, \quad q=n_{2}+1, \quad n=p+q-2 .
$$

The conformal linear transformation group $C O(Q)\left(\subset G L\left(n_{1}+n_{2}, \mathbb{R}\right)\right)$ acts on the isotropic cone $C\left(\subset \mathbb{R}^{n_{1}+n_{2}}\right)$, and then induces a unitary representation on $L^{2}(C)$ (see $(2.3 .10)-(2.3 .12)$ for a concrete formula). Much more than that, this action on $L^{2}(C)$ can be extended to a unitary representation $\pi$ of the indefinite orthogonal group $G=O\left(n_{1}+1, n_{2}+1\right)$. To explain its idea, we also recall another geometric model, namely, the conformal model. 
The conformal model of the minimal representation $\pi$ is realized, for example, in the solution space to the ultra-hyperbolic equation

$$
\left(\frac{\partial^{2}}{\partial x_{1}^{2}}+\cdots+\frac{\partial^{2}}{\partial x_{n_{1}}^{2}}-\frac{\partial^{2}}{\partial x_{n_{1}+1}^{2}}-\cdots-\frac{\partial^{2}}{\partial x_{n}^{2}}\right) u=0
$$

in $\mathcal{S}^{\prime}\left(\mathbb{R}^{n}\right)$ (tempered distributions), and also in the solution space

$$
\widetilde{\Delta}_{S^{n_{1}} \times S^{n_{2}}} v=0 \quad \text { on } S^{n_{1}} \times S^{n_{2}}
$$

where $\widetilde{\Delta}_{S^{n_{1}} \times S^{n_{2}}}=\Delta_{S^{n_{1}}}-\Delta_{S^{n_{2}}}-\left(\frac{n_{1}-1}{2}\right)^{2}+\left(\frac{n_{2}-1}{2}\right)^{2}$ is the Yamabe operator on the direct product manifold $S^{n_{1}} \times S^{n_{2}}$ equipped with the pseudoRiemannian structure of signature $\left(n_{1}, n_{2}\right)$. These two models are isomorphic to each other by the general theory of conformal geometry, and the intertwining operator is given by the 'twisted pull-back' $\widetilde{\Psi}^{*}$ of the conformal map $\Psi: \mathbb{R}^{n_{1}+n_{2}} \rightarrow S^{n_{1}} \times S^{n_{2}}$, the inverse of the stereographic projection (see [43] for an elementary account). Then, taking the Fourier transform $\mathcal{F}_{\mathbb{R}^{n}}$ of the conformal model, we get the Schrödinger model $L^{2}(C)$.

The intertwining operator $\mathcal{T}: L^{2}(C) \rightarrow \operatorname{Sol}\left(\widetilde{\Delta}_{S^{n_{1}} \times S^{n_{2}}}\right)$ is defined in Section 2.2 so that the following diagram commutes:

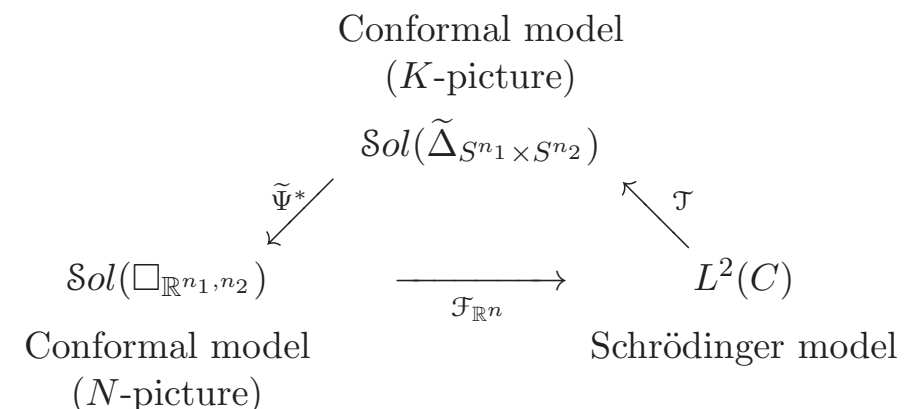

Diagram 1.7.1

We remark that the isotropic cone $C$ is defined as a hypersurface in $\mathbb{R}^{n}=\mathbb{R}^{n_{1}+n_{2}}$, and the group $G=O\left(n_{1}+1, n_{2}+1\right)$ cannot act (non-trivially) on $C$. (In fact, any (non-trivial) $G$-space is of dimension at least $n_{1}+n_{2}=$ $\operatorname{dim} C+1$.)

In the $L^{2}$-model $L^{2}(C)$ of the indefinite orthogonal group $G$, the action of a maximal parabolic subgroup

$$
\overline{P^{\max }} \simeq \mathbb{Z}_{2} \cdot C O(Q) \ltimes \mathbb{R}^{n_{1}+n_{2}}
$$


on $L^{2}(C)$ is of a simple form (see (2.3.10) $-(2.3 .13)$ for definition). This parabolic subgroup $\overline{P^{\max }}$ plays a similar role of the Siegel parabolic subgroup $P_{\text {Siegel }}$, and analogous results to the properties C1, C $\mathbf{2}$ and $\mathbf{C} \mathbf{3}$ hold (see Section 2.3). If we set

$$
w_{0}:=I_{n_{1}+1, n_{2}+1},
$$

then $w_{0}$ sends $\overline{P^{\max }}$ to the opposite parabolic subgroup $P^{\max }$, and $G$ is generated by $w_{0}$ and $\overline{P^{\max }}$.

In light of the Bruhat decomposition $G=\overline{P^{\max }} \amalg \overline{P^{\max }} w_{0} \overline{P^{\max }}$, we can get directly the concrete form of the action of the whole group $G$ once we know $\pi\left(w_{0}\right)$ explicitly.

In the degenerate case $\left(n_{1}, n_{2}\right)=(2,0), G=O(3,1)$ is locally isomorphic to $S L(2, \mathbb{C})$ acting on $\mathbb{R}^{2+0}(\simeq \mathbb{C})$ as Möbius transforms (linear fractional transforms), and $w_{0}$ acts on $\mathbb{C}$ as the conformal inversion

$$
\mathbb{C} \rightarrow \mathbb{C}, \quad z \mapsto-\frac{1}{z}
$$

Although $\left(n_{1}, n_{2}\right)=(2,0)$ is beyond the parameter in our consideration of the representation, this feature of the conformal inversion $w_{0}$ is valid for any $\left(n_{1}, n_{2}\right)$ (see Section 2.5 for a list of key properties of this element $w_{0}$ ).

In this book, we establish an analogous result to $\mathrm{C} 4$ for $G=O\left(n_{1}+\right.$ $\left.1, n_{2}+1\right)$, that is, we find the unitary operator $\pi\left(w_{0}\right)$ on $L^{2}(C)$ for the conformal inversion $w_{0}$.

Then, here is our main result in this context.

Main Theorem (see Theorem 5.1.1). Let $n_{1}, n_{2} \geq 1$ and $n=n_{1}+n_{2} \geq 4$ is even. Then the unitary operator $\pi\left(w_{0}\right): L^{2}(C) \rightarrow L^{2}(C)$ takes the form:

$$
\pi\left(w_{0}\right)=\mathcal{F}_{C},
$$

where $\mathcal{F}_{C}$ is the involutive unitary operator given in Section 1.2. That is,

$$
\pi\left(w_{0}\right) u(x)=\int_{C} K\left(x, x^{\prime}\right) u\left(x^{\prime}\right) d \mu\left(x^{\prime}\right), \quad u \in L^{2}(C),
$$

where the distribution kernel $K\left(x, x^{\prime}\right)$ is given in Theorem 1.3.1.

As obvious corollaries of the representation theoretic interpretation of $\mathcal{F}_{C}$ as above, we have:

Corollary 1.7.1. (Plancherel and inversion formulas, see Corollaries 5.1 .2 and 5.1.3)

$$
\begin{aligned}
\left\|\mathcal{F}_{C} u\right\|_{L^{2}(C)} & =\|u\|_{L^{2}(C)} & & \text { for } u \in L^{2}(C), \\
\mathcal{F}_{C}^{-1} & =\mathcal{F}_{C} & & \text { on } L^{2}(C) .
\end{aligned}
$$


The inversion formula $\mathcal{F}_{C}^{-1}=\mathcal{F}_{C}$ implies that the following relation

$$
\begin{aligned}
\left(\mathcal{F}_{C} u\right)(x) & =\int_{C} K\left(x, x^{\prime}\right) u\left(x^{\prime}\right) d \mu\left(x^{\prime}\right), \\
u(x) & =\int_{C} K\left(x, x^{\prime}\right)\left(\mathcal{F}_{C} u\right)\left(x^{\prime}\right) d \mu\left(x^{\prime}\right)
\end{aligned}
$$

is reciprocal. Such an inversion formula is sometimes referred to as a reciprocal formula (see Titchmarsh [71, for this terminology in a general setting).

It is noteworthy that Corollary 1.7.1 is stated without any language of group theory. It would be an interesting problem to give a straightforward proof of Corollary 1.7.1 from the definition of Bessel distributions (1.3.1)(1.3.3) without group theory.

By M2 and M4, $\pi$ is a non-highest weight, minimal representation if and only if $n_{1}, n_{2}>1$ and $n>4$. Therefore, we have discovered another mysterious phenomenon:

Corollary 1.7.2. The kernel $K\left(x, x^{\prime}\right)$ of the unitary inversion $\pi\left(w_{0}\right)$ is a not locally integrable function if and only if $\pi$ is a non-highest weight, minimal representation.

The highest weight module case $\left(n_{1}=1\right.$ or $\left.n_{2}=1\right)$

In the case $n_{2}=1$ (likewise $n_{1}=1$ ), $\pi$ splits into the direct sum of a highest weight module $\pi_{+}$and a lowest weight module $\pi_{-}$when restricted to the identity component $G_{0}=S O_{0}\left(n_{1}+1,2\right)$ of $G=O\left(n_{1}+1,2\right)$ according to the decomposition (1.3.4). Both $\pi_{+}$and $\pi_{-}$are minimal representations of $G_{0}$.

We note that $G$ is the conformal group $O\left(n_{1}+1,2\right)$ of the Minkowski space $\mathbb{R}^{n_{1}, 1}$, namely, the Euclidean space $\mathbb{R}^{n_{1}+1}$ equipped with the flat Lorentz metric of signature $\left(n_{1}, 1\right)$. In this case our representation $\pi$ has been studied also in physics. The minimal representation $\pi_{+}$may be interpreted as the symmetry of the solution space to the mass-zero spin-zero wave equation. The representation $\pi_{+}$arises also on the Hilbert space of bound states of the Hydrogen atom.

It is known that highest weight representations can be extended to holomorphic semigroups of a complexified Lie group $G_{\mathbb{C}}$. This theory has been initiated by Olshanski [58] and Stanton [66], among others, in connection with the Gelfand-Gindikin program to realize a family of representations in a geometrically unified manner. In this context the unitary operator $\pi\left(w_{0}\right)$ may be regarded as the boundary value of a holomorphic semigroup. We then ask an explicit form of the holomorphic semigroup. This idea was 
first exploited by Howe 35] for the Weil representation, where he showed that $\varpi\left(w_{0}\right)\left(=e^{\frac{\sqrt{-1} n \pi}{4}} \mathcal{F}_{\mathbb{R}^{n}}\right)$ is given as the boundary value of the Hermite semigroup, i.e., the holomorphic semigroup with the Mehler kernel (see also Folland 18 for an exposition). The same idea also works in our setting of $O\left(n_{1}+1, n_{2}+1\right)$ with $n_{2}=1$, and the explicit formula of a certain holomorphic semigroup (the 'Laguerre semigroup') yields the formula of the unitary inversion operator $\pi\left(w_{0}\right)$ by taking its boundary value in [44, 46]. In this book, as a special case (i.e. $n_{2}=1$ ) of Theorem [5.1.1, we give a new proof of the formula of $\pi\left(w_{0}\right)$.

\subsection{Uncertainty relation - inner products and $G$ - actions}

In this section, we consider the models of representations in the previous section in a more general setting, and formalize two representation theoretic questions (see Problem 1.8.1).

Let $P=L N=M A N$ be a parabolic subgroup of a real reductive Lie group $G, \bar{P}=L \bar{N}$ its opposite parabolic subgroup, and $\mathfrak{g}=\mathfrak{n}+\mathfrak{l}+\overline{\mathfrak{n}}$ the corresponding Gelfand-Naimark decomposition of the Lie algebra $\mathfrak{g}$. Assume that the nilradical $\mathfrak{n}$ is abelian, and in particular $P$ is a maximal parabolic subgroup.

Take a (non-unitary) one-dimensional representation $\chi: L \rightarrow \mathbb{C}^{\times}$, and consider the induced representation $W:=\operatorname{Ind}_{P}^{G}(\chi \otimes \mathbb{C})$. Then the space $W^{\infty}$ consisting of its smooth vectors can be regarded as a subspace of $\left(C^{\infty} \cap \mathcal{S}^{\prime}\right)(\overline{\mathfrak{n}})$ by the restriction to $\overline{\mathfrak{n}} \simeq \bar{N} \subset G / P$. Here, $\mathcal{S}^{\prime}(\overline{\mathfrak{n}})$ denotes the space of tempered distributions on $\overline{\mathfrak{n}}$ (regarded as the Euclidean space). Then, taking the Euclidean Fourier transform, we have

$$
W^{\infty} \subset \mathcal{S}^{\prime}(\overline{\mathfrak{n}}) \underset{\mathcal{F}}{\stackrel{\sim}{\longrightarrow}} \mathcal{S}^{\prime}(\mathfrak{n})
$$

where $\mathfrak{n}$ is identified with the dual space of $\mathfrak{n}$. We let $G$ act on $\mathcal{F}\left(W^{\infty}\right)$ through $\mathcal{F}$. This $G$-action cannot be extended to $\mathcal{S}^{\prime}(\mathfrak{n})$, but its restriction to the parabolic subgroup $\bar{P}=L \bar{N}$ can be extended to $\mathcal{S}^{\prime}(\mathfrak{n})$ because $\bar{P}$ acts on $\mathcal{S}^{\prime}(\mathfrak{n})$ just by translations and multiplications of unitary characters. Let $w_{0}$ be an element of $K$ such that $w_{0} L w_{0}^{-1}=L$ and $w_{0} N w_{0}^{-1}=\bar{N}$ (a conformal inversion).

Now we consider the following setting:

1) Let $(\pi, \mathcal{H})$ be an irreducible unitary representation of $G$, such that the 
underlying $\left(\mathfrak{g}_{\mathbb{C}}, K\right)$-module $\mathcal{H}_{K}$ is a subrepresentation of $W$, and the Hilbert space $\mathcal{H}$ is realized in $\mathcal{S}^{\prime}(\overline{\mathfrak{n}})$.

2) Let $C$ be an $L$-orbit in $\mathfrak{n}$, having an $M$-invariant measure $d \mu$ such that $L^{2}(C, d \mu) \subset \mathcal{S}^{\prime}(\mathfrak{n})$. Further, $\bar{P}=L \bar{N}$ leaves $L^{2}(C, d \mu)$ invariant, and acts as a unitary representation.

3) $\mathcal{F}(\mathcal{H})=L^{2}(C, d \mu)$.

We note that the condition (2) determines the absolute value $|\chi(a)|$ for $a \in A$. As we mentioned, the $\bar{P}$-action on $L^{2}(C)$ is given just by translations and multiplications of unitary characters. Since $G$ is generated by $\bar{P}$ and $w_{0}$, the action $\pi$ of the whole group $G$ on $L^{2}(C, d \mu)$ is determined by finding the formula of $\pi\left(w_{0}\right)$.

The (Euclidean) Fourier transform $\mathcal{F}$ transfers the defining ideal for the affine variety $\bar{C}$ in $\mathfrak{n}$ to the system, to be denoted by $\mathcal{M}$, of differential equations on $\overline{\mathfrak{n}}$ such that the space $\mathcal{H}$ is contained in the solution space:

$$
\mathcal{S o l}(\mathcal{M}):=\left\{f \in \mathcal{S}^{\prime}(\overline{\mathfrak{n}}): P f=0 \text { for any } P \in \mathcal{M}\right\} .
$$

In the previous example, $\mathfrak{n} \simeq \mathbb{R}^{n}, C$ is the isotropic cone, and the system $\mathcal{M}$ is generated by $\square_{\mathbb{R}^{n_{1}, n_{2}}}=\frac{\partial^{2}}{\partial x_{1}^{2}}+\cdots+\frac{\partial^{2}}{\partial x_{n_{1}}^{2}}-\frac{\partial^{2}}{\partial x_{n_{1}+1}^{2}}-\cdots-\frac{\partial^{2}}{\partial x_{n}^{2}}$.

Thus, we have two models of the irreducible unitary representation with the following nature:

Solution model on $\mathcal{S} o l(\mathcal{M})$ in $\mathcal{S}^{\prime}(\overline{\mathfrak{n}})$.

- The 'intrinsic inner product' on the Hilbert space $\mathcal{H}(\subset \operatorname{Sol}(\mathcal{M}))$ is not clear.

- The $G$-action on $\mathcal{H}$, to be denoted by $\varpi(g)$, is simple (essentially, the translations by the Möbius transform of $G$ on $G / P \fallingdotseq \overline{\mathfrak{n}})$.

$L^{2}$-model on $L^{2}(C, d \mu) \subset \mathcal{S}^{\prime}(\mathfrak{n})$.

- The inner product on the Hilbert space $L^{2}(C, d \mu)$ is very clear.

- The $G$-action on $L^{2}(C, d \mu)$ is not simple except for the $\bar{P}$-action.

Now, we have a kind of 'uncertainty relation' in the sense that it is hard to find a single model having explicit descriptions of both $G$-actions and inner products. This feature in the above two models is symbolically summarized as follows: 


\begin{tabular}{l|cc} 
& $\operatorname{Sol}(\mathcal{M})$ & $L^{2}(C, d \mu)$ \\
\hline inner product & $?$ & simple \\
G-action & simple & $?$
\end{tabular}

Then, we ask:

Problem 1.8.1.

1) (conserved quantity for solution model) Find an intrinsic inner product on the solution space $\operatorname{Sol}(\mathcal{M})$ that is invariant by $G$.

2) (generalization of the Fourier-Hankel transform for $L^{2}$-model) Find an explicit formula for the unitary inversion operator $\pi\left(w_{0}\right)$.

1) Solution model. In order to clarify the meaning of 'intrinsic inner product', we list three approaches to describe the inner product on $\mathcal{H}$ in $\operatorname{Sol}(\mathcal{M})$.

1-a) (Parseval type formula) Describe the inner product on $\mathcal{H}$ according to the $K$-type decomposition.

1-b) (Green function) Give an integral expression of the solutions to $\mathcal{M}$, and describe the inner product on $\mathcal{H}$ by means of the integral expression.

1-c) (Conserved quantities) Find an inner product formula in terms of only solutions.

The approaches (1-a) and (1-b) give explicit inner products in a sense and are usually sufficient for representation theoretic purposes (e.g. showing the unitarizability), however, do not give an intrinsic formula in the sense that the formula depends on the $K$-type decomposition or on the integral expression of solutions. The approach (1-c) seeks for an intrinsic formula based purely on solutions. Here are a few remarks on (1-a)-(1-c) in order.

The approach (1-a) is algebraic. We note that $(G, L)$ forms a reductive symmetric pair under the assumption that the nilpotent radical $\mathfrak{n}$ is abelian. Consequently, $(K, L \cap K)$ is a compact symmetric pair, and therefore $\operatorname{Ind}_{P}^{G}(\chi \otimes \mathbb{C})$ is $K$-multiplicity free. Then, the unitary inner product on $\mathcal{H}$ is a scalar multiple of the $L^{2}$-inner product on $L^{2}(K / L \cap K)$ on each $K$ type by Schur's lemma. Thus, the unitary inner product on $\mathcal{H}$ is expressed by the 'weight function'

$$
m: \widehat{K}_{L \cap K} \rightarrow \mathbb{R}_{+},
$$


where $\widehat{K}_{L \cap K}=\left\{\tau \in \widehat{K}: \operatorname{Hom}_{K}\left(\tau, L^{2}(K / L \cap K)\right) \neq 0\right\}$ is explicitly known by the Cartan-Helgason theorem. The weight function $m$ for unitarizable subquotients have been computed for a number of degenerate principal series representations, especially since the influential paper [36] by Howe and Tan. More generally, discretely decomposable branching laws for non-compact subgroups $H$ give an extension of the approach (1-a) (see [48]).

The approaches (1-b) and (1-c) are analytic. The integral expression of the solutions in (1-b) corresponds to the Knapp-Stein intertwining operator $A$ in representation theory. The $G$-invariance of the resulting bilinear form $f_{1}, f_{2} \mapsto\left(f_{1}, A f_{2}\right)$ is clear, and the positivity of the bilinear form implies the unitarizability of Image $A$. This formula of the inner product is explicit, however, the formula is not written directly in terms of solutions. In fact, it is non-trivial to find a preimage $f$ such that $u=A f$ when a solution $u$ is given.

What we seek for in (1-c) is to describe directly the inner product on solutions. The energy for the wave equation is invariant under the time translation, and is a classic example of conserved quantities. The unitarizability of a solution space to $\mathcal{M}$ predicts the existence of positive definite conserved quantities. If the solution space is an irreducible $G$-module, then such conserved quantities must be unique up to scalar. This is what we call the 'intrinsic inner product' on the solution space. The uniqueness and the existence is predicted by representation theory. Finding its explicit form would be a challenging problem in analysis, arising naturally from representation theory.

2) $L^{2}$-model. The existence of $L^{2}$-models of small representations has been found for some other reductive groups. See [8, 32, 74] for unitary highest weight representations, [13] for spherical cases by using Jordan algebras; 62 for $S O(4,3)$, 73 . for minimal representations of general reductive groups by using amalgamation of maximal parabolic subgroups.

In the setting that we discussed as $L^{2}$-model, there is a simple action of a parabolic subgroup $\bar{P}$ on $L^{2}(C)$, and we know the existence of the $G$-action on $L^{2}(C)$ by some other reasons. On the other hand, it is often the case that the missing piece is the explicit global formula on how to extend the action on $L^{2}(C)$ from $\bar{P}$ to the whole group $G$.

Our subject stated in Problem 1.8.1 (2) is to fill this missing piece by finding the formula of the 'unitary inversion' $\pi\left(w_{0}\right)$ for the conformal inversion element $w_{0} \in G$. We remark that the operator $\pi\left(w_{0}\right)$ in the $L^{2}$-model can be written as

$$
\pi\left(w_{0}\right)=\mathcal{F} \circ \varpi\left(w_{0}\right) \circ \mathcal{F}^{-1},
$$


where $\varpi\left(w_{0}\right)$ is a simple action on the solution model $\operatorname{Sol}(\mathcal{M})$. However, the right-hand side of the formula (1.8.1) does not give a solution to Problem 1.8.1 (2) readily, because it is not easy to carry out the computation of the composition of integral forms in general.

On the other hand, finding the formula of the unitary inversion $\pi\left(w_{0}\right)$ in the $L^{2}$ model $L^{2}(C)$ has a significant meaning. To see this, we recall that there is a Bruhat decomposition $G=\bar{P} \cup \bar{P} w_{0} \bar{P}$. Therefore, once we get a formula of $\pi\left(w_{0}\right)$, then the whole group action of $G$ can be written by using $\pi\left(w_{0}\right)$ at most once (without any further composition of integral operators). Thus, the formula of $\pi\left(w_{0}\right)$ is critical for finding the global formula of the whole action of $G$.

We have seen in Sections 1.6 and 1.7 that Problem 1.8.1 (2) is settled for the Weil representation for the metaplectic group $M p(n, \mathbb{R})$ and the minimal representation of the indefinite orthogonal group $O\left(n_{1}+1, n_{2}+1\right)$, respectively. More generally, in the case that $(\pi, \mathcal{H})$ is a minimal representation of a reductive group $G$, we expect that the operator could be described by means of some 'special function' of one variable.

\subsection{Special functions and minimal representations}

Yet another theme is special functions.

In this book, we shall see special functions arise from the minimal representation. For example, $K$-Bessel functions appear as the radial part of $K$-finite vectors in the Schrödinger model $L^{2}(C)$. Meijer's $G$-functions appear as the radial part of the integral kernel of $\mathcal{F}_{C}$. Appell's hypergeometric functions bridge two models of the minimal representation, namely, the Schrödinger model and the conformal model. All together, we develop a new line of investigation on various special functions in connection with the minimal representation.

\section{The 'radial part' of the unitary inversion $\mathcal{F}_{C}$}

We begin with the (Euclidean) Fourier transform $\mathcal{F}_{\mathbb{R}^{n}}$ as an illustrative example. As we already discussed, this corresponds to the unitary inversion operator for the (original) Schrödinger model of the Weil representation of $M p(n, \mathbb{R})$.

According to the polar coordinate

$$
\mathbb{R}_{+} \times S^{n-1} \rightarrow \mathbb{R}^{n}, \quad(r, \omega) \mapsto r \omega,
$$


we have a unitary equivalence:

$$
\begin{aligned}
L^{2}\left(\mathbb{R}^{n}\right) & \simeq L^{2}\left(\mathbb{R}_{+}, r^{n-1} d r\right) \widehat{\otimes} L^{2}\left(S^{n-1}\right) \\
& \simeq \sum_{l=0}^{\infty} L^{2}\left(\mathbb{R}_{+}, r^{n-1} d r\right) \otimes \mathcal{H}^{l}\left(\mathbb{R}^{n}\right),
\end{aligned}
$$

where $\mathcal{H}^{l}\left(\mathbb{R}^{n}\right)$ is the space of spherical harmonics of degree $l,\left\{\varphi \in C^{\infty}\left(S^{n-1}\right)\right.$ : $\left.\Delta_{S^{n-1}} \varphi=-l(l+n-2) \varphi\right\}$ (see Appendix 7.5$)$. Here, $\widehat{\otimes}$ stands for the Hilbert completion of the tensor product space, and $\sum^{\oplus}$ stands for the Hilbert completion of an algebraic direct sum.

Correspondingly to the direct sum decomposition (1.9.1), the Fourier transform $\mathcal{F}_{\mathbb{R}^{n}}$ is decomposed as

$$
\mathcal{F}_{\mathbb{R}^{n}}=\sum_{l=0}^{\infty} T_{l} \otimes \mathrm{id} .
$$

Here, $T_{l}$ is the Hankel transform of the following form (see Remark 4.1.3):

$$
\left(T_{l} f\right)(r)=\frac{c}{r^{n}} \int_{0}^{\infty} x^{\frac{n}{2}} J_{\frac{n-2+2 l}{2}}(x) f\left(\frac{x}{r}\right) d x .
$$

Next, we consider our minimal representation of $O\left(n_{1}+1, n_{2}+1\right)$ realized on $L^{2}(C)$. Then, the bipolar coordinate on the isotropic cone $C$,

$$
\mathbb{R}_{+} \times S^{n_{1}-1} \times S^{n_{2}-1} \rightarrow C
$$

induces a unitary equivalence

$$
\begin{aligned}
L^{2}(C) & \simeq L^{2}\left(\mathbb{R}_{+}, \frac{1}{2} r^{n-3} d r\right) \widehat{\otimes} L^{2}\left(S^{n_{1}-1}\right) \otimes L^{2}\left(S^{n_{2}-1}\right) \\
& \simeq \sum_{l, k=0}^{\infty} L^{2}\left(\mathbb{R}_{+}, \frac{1}{2} r^{n-3} d r\right) \otimes \mathcal{H}^{l}\left(\mathbb{R}^{n_{1}}\right) \otimes \mathcal{H}^{k}\left(\mathbb{R}^{n_{2}}\right) .
\end{aligned}
$$

Then, the unitary inversion operator $\mathcal{F}_{C}$ is decomposed as

$$
\mathcal{F}_{C}=\sum_{l, k=0}^{\infty} T_{l, k} \otimes \mathrm{id} \otimes \mathrm{id},
$$

where $T_{l, k}$ is a unitary operator on $L^{2}\left(\mathbb{R}_{+}, \frac{1}{2} r^{n-3} d r\right)$. The unitary operators $T_{l, k}$ may be regarded as a generalization of Hankel transforms.

It turns out that the kernel function of $T_{l, k}$ is real analytic (see Theorem 1.9.1 below). This is a good contrast to the fact that the unitary operator $\mathcal{F}_{C}$ on $L^{2}(C)$ is given by a distribution kernel for general $n_{1}, n_{2}>1$ (see Theorem 1.3.1). 
Theorem 1.9.1 (see Theorem 4.1.1). Let $G_{04}^{20}$ be Meijer's $G$-function (see Appendix 7.6 for definition), and we define a real analytic function $K_{l, k}$ by

$$
K_{l, k}(t):=4(-1)^{a+\frac{n_{1}+k+l}{2}} G_{04}^{20}\left(t^{2} \mid \frac{l+k}{2}, a+2, \frac{-n_{1}-n_{2}+4-l-k}{2}, b+2\right),
$$

where

$$
a:=\max \left(\frac{-n_{1}-l+k}{2}, \frac{-n_{2}+l-k}{2}\right), b:=\min \left(\frac{-n_{1}-l+k}{2}, \frac{-n_{2}+l-k}{2}\right) .
$$

Then, we have

$$
\left(T_{l, k} f\right)(r)=\frac{1}{2} \int_{0}^{\infty} K_{l, k}\left(r r^{\prime}\right) f\left(r^{\prime}\right) r^{\prime n-3} d r^{\prime}
$$

It is noteworthy that Meijer's $G$-functions arise in the representation theory of reductive Lie groups. We observe the Casimir operator of a maximal compact subgroup $K$ acts on $L^{2}(C)$ as a fourth order differential operator. Correspondingly, Meijer's $G$-functions $G_{04}^{20}\left(x \mid b_{1}, b_{2}, b_{3}, b_{4}\right)$ solve ordinary differential equations of order four (see (7.6.6)):

$$
\prod_{j=1}^{4}\left(x \frac{d}{d x}-b_{j}\right) u=0 .
$$

In the case $n_{1}=1$ (or $n_{2}=1$ ), our minimal representation $\pi$ is a direct sum of a highest weight representation and a lowest weight representation. In this case, the kernels $K_{l, k}$ collapse to Bessel functions, and the unitary operators $T_{l, k}$ are reduced to Hankel transforms.

The group law $w_{0}^{2}=1$ in $G$ implies $\pi\left(w_{0}\right)^{2}=\mathrm{id}$, and consequently, $T_{l, k}^{2}=\mathrm{id}$ for every $l, k \in \mathbb{N}$. Hence, Theorem 1.9 .1 gives a group theoretic proof for the Plancherel and reciprocal formulas on Meijer's $G$-transforms which were first proved by C. Fox [19] by a completely different method.

Corollary 1.9.2 (see Corollary 4.1.4). Let $b_{1}, b_{2}, \gamma$ be half-integers such that $b_{1} \geq 0, \gamma \geq 1, \frac{1-\gamma}{2} \leq b_{2} \leq \frac{1}{2}+b_{1}$. Then, the integral transform

$$
S_{b_{1}, b_{2}, \gamma}: f(x) \mapsto \frac{1}{\gamma} \int_{0}^{\infty} G_{04}^{20}\left((x y)^{\frac{1}{\gamma}} \mid b_{1}, b_{2}, 1-\gamma-b_{1}, 1-\gamma-b_{2}\right) f(y) d y
$$

is a unitary operator on $L^{2}\left(\mathbb{R}_{+}\right)$.

Corollary 1.9.3 (see Corollary 4.1.5). The unitary operator $S_{b_{1}, b_{2}, \gamma}$ is of order two in $L^{2}\left(\mathbb{R}_{+}\right)$, that is, $\left(S_{b_{1}, b_{2}, \gamma}\right)^{-1}=S_{b_{1}, b_{2}, \gamma}$. 
A special case of the above corollaries (i.e. $n_{2}=1$ case) yields the classic formulas of the Hankel transform (see Remark 4.1.6).

\section{$K$-finite vectors in $L^{2}(C)$}

By the general theory due to Vogan [76, $K$-types of minimal representations $\pi$ are indexed by a natural number $a=0,1,2, \ldots$ (by this property, $\pi$ is an example of the so-called ladder representation). In contrast to the conformal model on $\operatorname{Sol}\left(\widetilde{\Delta}_{S^{p-1} \times S^{q-1}}\right)$ where explicit $K$-finite vectors are given readily by spherical harmonics, it is not clear a priori what $K$-finite vectors look like in the Schrödinger model $L^{2}(C)$ because the whole group $K$ cannot act on the isotropic cone $C$.

Our idea is to compute explicitly the intertwining integral operator $\mathfrak{T}^{-1}$ between these two models in Diagram 1.7.1. Then, by using a reduction formula of Appell's hypergeometric functions, we have (loosely):

$$
\mathcal{T}^{-1} \text { (Gegenbauer polynomials) }=K \text {-Bessel functions }
$$

and prove the following result:

Theorem 1.9.4 (see Corollary 3.1.2). Let $n_{1} \geq n_{2}$. For $a=0,1,2, \ldots$,

$$
r^{a-\frac{n_{2}-1}{2}} K_{\frac{n_{2}-1}{2}}(2 r) \phi(w) \quad\left(\phi \in \mathcal{H}^{a}\left(\mathbb{R}^{n_{1}}\right)\right)
$$

is a $K$-finite vector in $L^{2}(C)$. In the $K$-type formula (see (2.1.6)), this vector belongs to the $K$-type $\mathcal{H}^{a}\left(\mathbb{R}^{n_{1}+1}\right) \otimes \mathcal{H}^{a+\frac{n_{1}-n_{2}}{2}}\left(\mathbb{R}^{n_{2}+1}\right)$.

In Theorem 1.9.4, the $a=0$ case corresponds to the minimal $K$-type, and was previously proved in [49, Theorem 5.8]. We note that $\pi$ is spherical if $n_{1}=n_{2}$. Even in the case of spherical representations, finding explicit forms of the $K$-fixed vectors in $L^{2}$-model is non-trivial. See [13] for similar formulas of the $K$-fixed vectors in $L^{2}$-models for some other groups by means of $K$-Bessel functions.

\subsection{Organization of this book}

This book is organized as follows. We review quickly the $L^{2}$-realization (a generalization of the classic Schrödinger model) of the minimal representation of $O(p, q)$ in the first half of Chapter 2, and develop a basic theory of fundamental differential operators on the isotropic cone $C$ in the latter half. Then we find some $K$-finite vectors on $L^{2}(C)$ explicitly by means of the $K$-Bessel function $K_{\nu}(z)$ in Chapter 3 . Chapter 4 is devoted entirely to the 
integral formula of the unitary operator $T_{l, k}$ on $L^{2}\left(\mathbb{R}_{+}, r^{p+q-5} d r\right)$ for double spherical harmonics expansions (see Theorem 4.1.1). In Chapter 5, building on the results of Chapter 4, we complete the proof of our main theorem (see Theorem 5.1.1). In order to make the proof readable as much as possible, we collect in Appendix the formulas and the properties of various special functions used in this book.

\subsection{Acknowledgements}

A large part of the results here was obtained while both authors were at the Research Institute for Mathematical Sciences, Kyoto. The materials for Sections 1.1 1.3 and $2.4-2.5$ were developed while the first author was participating in the program "Representation Theory, Complex Analysis and Integral Geometry" organized by B. Krötz and S. Gindikin at MaxPlanck-Institut für Mathematik, Bonn in 2007. We are very grateful to the colleagues and the staff for the wonderful atmosphere of research at these institutes.

The authors have benefited from various discussions with J. Faraut, S. Gindikin, S. Hansen, M. Kashiwara, T. Koorwinder, B. Ørsted, H. Sabourin, S. Sahi, P. Torasso, and D. Vogan.

Some of the results of this book were presented at the workshop on "Harmonic Analysis and Homogeneous Spaces" in honor of Professor G. van Dijk at Lorentz Center in Leiden in 2004, at the Faraut Seminar in Paris in 2005, at the workshop on "Representation Theory and Prehomogeneous Vector Spaces" at IRMA, Strasbourg in 2006, and at the workshop in Poitiers and at the Analysis Seminar of Aarhus University in 2007. We express our deep gratitude to the organizers of these workshops and seminars, and to the participants for helpful and stimulating comments on various occasions.

A part of the results here was announced in [45] with a sketch of proof.

The first author would like to thank Ms. Suenaga for indispensable help in preparing $\mathrm{AT}_{\mathrm{E} X}$ manuscript.

Notation: $\mathbb{R}_{+}:=\{x \in \mathbb{R}: x>0\}, \mathbb{N}:=\{0,1,2, \cdots\}$. 


\section{Chapter 2}

\section{Two models of the minimal representation of $O(p, q)$}

This chapter gives an account of the connection of the following two topics:

1) Analysis on the isotropic cone $C$ for commuting differential operators $P_{j}(1 \leq j \leq n)$ associated to the quadratic form of signature $\left(n_{1}, n_{2}\right)$ (see Introduction 1.1-1.3).

2) Minimal representation of the indefinite orthogonal group $O(p, q)$.

Throughout this chapter, we shall use the following notation:

$$
p=n_{1}+1, \quad q=n_{2}+1, \quad n=n_{1}+n_{2}=p+q-2 .
$$

The first half of this chapter is a review from [47, 49] about two concrete models of the minimal representation of the group $G=O(p, q)$, namely, the conformal model $\left(\varpi^{p, q},{\overline{V^{p, q}}}^{p}\right)$ using the Yamabe operator (2.1.1) in Section 2.1 and the $L^{2}$-model (the Schrödinger model) $\left(\pi, L^{2}(C)\right)$ in Section 2.2. In the terminology of representation theory of reductive Lie groups (e.g. [41, 78]), the former realization gives a subrepresentation (the $K$-picture, the $N$-picture, etc.) of a degenerate principal series representation, whereas the latter corresponds to the dual of the $N$-picture via the (Euclidean) Fourier transform $\mathcal{F}$.

The intertwining operator $\mathcal{T}$ between these two models will be given in (2.2.7), which is summarized as the following diagram:

$$
\begin{array}{ccc}
L^{2}(C) & \hookrightarrow & \mathcal{S}^{\prime}\left(\mathbb{R}^{p+q-2}\right) \\
\mathcal{T} & & \uparrow \mathcal{F} \\
K \text {-picture } & \underset{\widetilde{\Psi}^{*}}{\rightarrow} & N \text {-picture. }
\end{array}
$$


Here, $T$ is the identification map between functions on $C$ and distributions supported on $C$ by the canonical measure on $C, \widetilde{\Psi}^{*}$ is the $G$-intertwining operator between the $K$-picture and the $N$-picture, and is interpreted as the twisted pull-back for the conformal map $\Psi: \mathbb{R}^{p+q-2} \rightarrow S^{p-1} \times S^{q-1}$.

The latter half of this chapter is new. In Section 2.4, we analyze a commuting family of differential operators $P_{j}(b)(1 \leq j \leq n)$ of second order with parameter $b$ in $\mathbb{R}^{n}$, and prove that they are tangential to the isotropic cone $C$ if $b=1$. The resulting differential operators $P_{j}:=\left.P_{j}(1)\right|_{C}$ on $C_{0}^{\infty}(C)$ extend to self-adjoint operators on $L^{2}(C)$ (see Theorem 2.4.1). Thus, we get a family of commuting differential operators $P_{j}(1 \leq j \leq n)$ of second order, which we call fundamental differential operators on $C$. In Section 2.5, we see that the unitary inversion operator $\mathcal{F}_{C}=\pi\left(w_{0}\right)$ diagonalizes $P_{j}(1 \leq j \leq n)$ and that the intertwining relation of $P_{j}$ and the multiplication by coordinate function $x_{j}$ characterizes $\mathcal{F}_{C}$ up to scalar. Thus, we develop an abstract theory of the unitary operator $\mathcal{F}_{C}$ on $L^{2}(C)$ by taking the (Euclidean) Fourier transform $\mathcal{F}_{\mathbb{R}^{n}}$ on $L^{2}\left(\mathbb{R}^{n}\right)$ as a prototype (see Theorems $2.5 .2,2.5 .4,2.5 .5$ ).

\subsection{Conformal model}

This section summarizes the conformal model of the minimal representation of the indefinite orthogonal group $G=O(p, q)(p+q$ : even). The advantage of the conformal model is that the group action on the representation is simple and that its geometric idea is clear. Since this conformal model corresponds to a subrepresentation of the most degenerate principal series representations (with a very special parameter), the same representation can be studied also by the purely algebraic method of $(\mathfrak{g}, K)$-modules. See [6, 36, 50] in this direction. The same subrepresentation can also be captured by the theta correspondence arising from the dual pair $O(p, q) \cdot S L(2, \mathbb{R}) \subset$ $S p(p+q, \mathbb{R})$ (see [80]). Our approach in this section is geometric, and the basic reference here is [47]. See also [43] for an elementary exposition from viewpoints of conformal transformation groups.

The general geometric idea here is summarized as follows. Let $X$ be an $n$-dimensional manifold equipped with a Riemannian (or more generally, pseudo-Riemannian) structure $g$. Then, associated to $g$, we define:

$$
\begin{aligned}
& \kappa: \text { the scalar curvature on } X, \\
& \Delta_{X}: \text { the Laplace-Beltrami operator on } X, \\
& \widetilde{\Delta}_{X}:=\Delta_{X}+\frac{n-2}{4(n-1)} \kappa \quad \text { (Yamabe operator). }
\end{aligned}
$$


Then, although these objects depend on the (pseudo-)Riemannian structure $g$, the solution space

$$
\operatorname{Sol}\left(\widetilde{\Delta}_{X}\right):=\left\{f \in C^{\infty}(X): \widetilde{\Delta}_{X} f=0\right\}
$$

is conformally invariant, namely, if $\varphi: X \rightarrow X$ is a conformal diffeomorphism with a conformal factor $c_{\varphi} \in C^{\infty}(X)$ satisfying

$$
\varphi^{*} g=c_{\varphi}^{2} g
$$

then

$$
C^{\infty}(X) \rightarrow C^{\infty}(X), \quad f \mapsto c_{\varphi}^{-\frac{n-2}{2}} f \circ \varphi
$$

leaves $\operatorname{Sol}\left(\widetilde{\Delta}_{X}\right)$ invariant, and hence we get a representation of the conformal transformation group $\operatorname{Conf}(X, g)$ on $\operatorname{Sol}\left(\widetilde{\Delta}_{X}\right)$ (see [47, Theorem A]). The point here is that the above construction is functional under conformal maps, and in particular, if two pseudo-Riemannian manifolds are conformally equivalent (not necessarily isometric), then the resulting two representations are isomorphic.

A special case applied to pseudo-Riemannian manifolds which are conformally equivalent to flat pseudo-Riemannian space forms gives rise to the minimal representations of the indefinite orthogonal groups. Let us explain this specific case in more details.

We denote by $\mathbb{R}^{p, q}$ the Euclidean space $\mathbb{R}^{p+q}$ equipped with the pseudoRiemannian structure $g_{\mathbb{R}^{p, q}}$ of signature $(p, q)$ :

$$
d s^{2}=d x_{1}^{2}+\cdots+d x_{p}^{2}-d y_{1}^{2}-\cdots-d y_{q}^{2} .
$$

Then, the restriction of $d s^{2}$ to the submanifold

$$
\begin{aligned}
M & :=\left\{(x, y) \in \mathbb{R}^{p+q}:|x|=|y|=1, x \in \mathbb{R}^{p}, y \in \mathbb{R}^{q}\right\} \\
& \simeq S^{p-1} \times S^{q-1}
\end{aligned}
$$

is non-degenerate, and defines a pseudo-Riemannian structure on $M$ of signature $(p-1, q-1)$. Here,$|\cdot|$ stands for the usual Euclidean norm. The resulting pseudo-Riemannian structure $g_{M}$ on $M$ is nothing but the direct product of the standard unit sphere $S^{p-1}$ (positive definite metric) and the unit sphere $S^{q-1}$ equipped with the negative definite metric $((-1) \times$ the standard metric).

Then, the Yamabe operator $\widetilde{\Delta}_{M}$ of $M$ takes the following form (see [49, $(3,4,1)])$ :

$$
\widetilde{\Delta}_{M}=\Delta_{S^{p-1}}-\Delta_{S^{q-1}}-\left(\frac{p-2}{2}\right)^{2}+\left(\frac{q-2}{2}\right)^{2}
$$


where $\Delta_{S^{p-1}}$ and $\Delta_{S^{q-1}}$ are the Laplace-Beltrami operators on $S^{p-1}$ and $S^{q-1}$ respectively.

The indefinite orthogonal group $G=O(p, q)$ acts naturally on $\mathbb{R}^{p, q}$ as isometries. This action preserves the cone

$$
\Xi:=\left\{(x, y) \in \mathbb{R}^{p, q}:|x|=|y| \neq 0\right\}
$$

but does not preserve $M$. In order to let $G$ act on $M$, we set a function $\nu$ on $\mathbb{R}^{p, q}$ by

$$
\nu: \mathbb{R}^{p, q} \rightarrow \mathbb{R}, \quad(x, y) \mapsto|x| .
$$

If $v \in M(\subset \Xi)$ and $h \in G$, then $h \cdot v \in \Xi$, and consequently $\frac{h \cdot v}{\nu(h \cdot v)} \in M$. Thus, we can define the action of $G$ on $M$ :

$$
L_{h}: M \rightarrow M, \quad v \mapsto \frac{h \cdot v}{\nu(h \cdot v)} \quad(h \in G) .
$$

Then, we have $L_{h}^{*} g_{M}=\frac{1}{\nu(h \cdot v)^{2}} g_{M}$ at $T_{v} M$ and thus the diffeomorphism $L_{h}$ is conformal with respect to the pseudo-Riemannian metric on $M$. Conversely, any conformal diffeomorphism of $M$ is of the form $L_{h}$ for some $h \in G$ (see [42, Chapter IV]).

By the general theory (see $(2.1 .2)$ ) of conformal geometry, we can construct a representation, denoted by $\varpi^{p, q}$, of $G$ on the solution space to $\widetilde{\Delta}_{M}$ in $C^{\infty}(M)$ :

$$
V^{p, q}:=\operatorname{Sol} \widetilde{\Delta}_{M}=\left\{f \in C^{\infty}(M): \widetilde{\Delta}_{M} f=0\right\},
$$

where we set

$$
\left(\varpi^{p, q}\left(h^{-1}\right) f\right)(v):=\nu(h \cdot v)^{-\frac{p+q-4}{2}} f\left(L_{h} v\right),
$$

for $h \in G, v \in M$, and $f \in V^{p, q}$. The following theorem was proved in [49] in this geometric framework. There are also algebraic proofs (see Remark 2.1.2).

Fact 2.1.1 (see [49, Theorem 3.6.1]). Let $p, q \geq 2$ and $p+q \geq 6$ be even.

1) (irreducibility) $\left(\varpi^{p, q}, V^{p, q}\right)$ is an irreducible unitary representation of $G$.

2)(unitarizability) There exists a $G$-invariant inner product ( , $)_{M}$ on $V^{p, q}$. Such a G-invariant inner product is unique up to a scalar multiple, and we shall normalize it in (2.1.7).

We write $\overline{V^{p, q}}$ for the Hilbert completion of $V^{p, q}$, and use the same letter $\varpi^{p, q}$ to denote the resulting irreducible unitary representation. 
3) (K-type formula) Let $K \simeq O(p) \times O(q)$ be a maximal compact subgroup of $G$. Then, the restriction of $\left(\varpi^{p, q}, \overline{V^{p, q}}\right)$ to $K$ decomposes into irreducible representations of $K$ as follows:

$$
\overline{V^{p, q}} \simeq \sum_{\substack{a+\frac{p-q}{2}=b, a, b \in \mathbb{N}}}^{\infty} \mathcal{H}^{a}\left(\mathbb{R}^{p}\right) \otimes \mathcal{H}^{b}\left(\mathbb{R}^{q}\right) .
$$

Here, $\mathcal{H}^{a}\left(\mathbb{R}^{p}\right)$ denotes the irreducible representation of $O(p)$ on the space of spherical harmonics of degree a (see Section 7.5).

4) (Parseval-Plancherel formula) On each $K$-type $\mathcal{H}^{a}\left(\mathbb{R}^{p}\right) \otimes \mathcal{H}^{b}\left(\mathbb{R}^{q}\right)$ for $(a, b) \in \mathbb{N}^{2}$ such that $a+\frac{p-q}{2}=b$, or equivalently, $a+\frac{p-2}{2}=b+\frac{q-2}{2}$, the unitary inner product $(\cdot, \cdot)_{M}$ is of the form:

$$
(F, F)_{M}=\left(a+\frac{p-2}{2}\right)\|F\|_{L^{2}(M)}^{2} .
$$

Next, we consider the following injective map (see [49, (2.8.2)]) by

$$
\Psi: \mathbb{R}^{p+q-2} \rightarrow M, \quad z \mapsto \tau(z)^{-1} \iota(z),
$$

where for $z=\left(z^{\prime}, z^{\prime \prime}\right) \in \mathbb{R}^{p-1} \oplus \mathbb{R}^{q-1}$ we set

$$
\begin{aligned}
& \tau(z):=\left(1+\left(\frac{\left|z^{\prime}\right|+\left|z^{\prime \prime}\right|}{z}\right)^{2}\right)^{\frac{1}{2}}\left(1+\left(\frac{\left|z^{\prime}\right|-\left|z^{\prime \prime}\right|}{2}\right)^{2}\right)^{\frac{1}{2}} \\
& \iota: \mathbb{R}^{p+q-2} \rightarrow \mathbb{R}^{p+q},\left(z^{\prime}, z^{\prime \prime}\right) \mapsto\left(1-\frac{\left|z^{\prime}\right|^{2}-\left|z^{\prime \prime}\right|^{2}}{4}, z^{\prime}, z^{\prime \prime}, 1+\frac{\left|z^{\prime}\right|^{2}-\left|z^{\prime \prime}\right|^{2}}{4}\right) .
\end{aligned}
$$

Then, $\Psi$ is a conformal map such that $\Psi^{*} g_{M}=\tau(z)^{-2} g_{\mathbb{R}^{p-1, q-1}}$. According to [47, Definition 3.4], the twisted pull-back $\widetilde{\Psi}^{*}$ of the conformal map $\Psi$ is a linear map

$$
\widetilde{\Psi}^{*}: C^{\infty}(M) \rightarrow C^{\infty}\left(\mathbb{R}^{p+q-2}\right)
$$

given by

$$
\left(\widetilde{\Psi}^{*} f\right)(z):=\tau(z)^{-\frac{p+q-4}{2}}(f \circ \Psi)(z) .
$$

The image $M_{+}$of $\Psi$ is roughly the half of $M$ :

$$
M_{+}:=\left\{u=\left(u_{0}, u^{\prime}, u^{\prime \prime}, u_{p+q-1}\right) \in M: u_{0}+u_{p+q-1}>0\right\} .
$$

We note that $\Psi$ induces a conformal compactification of the flat space $\mathbb{R}^{p-1, q-1}$ :

$$
\mathbb{R}^{p-1, q-1} \hookrightarrow\left(S^{p-1} \times S^{q-1}\right) / \sim \mathbb{Z}_{2},
$$


where $\sim \mathbb{Z}_{2}$ denotes the equivalence relation in $M=S^{p-1} \times S^{q-1}$ defined by $u \sim-u$.

The inverse of $\Psi: \mathbb{R}^{p+q-2} \stackrel{\sim}{\rightarrow} M_{+}$is given by

$$
\Psi^{-1}\left(u_{0}, u^{\prime}, u^{\prime \prime}, u_{p+q-1}\right)=\left(\frac{u_{0}+u_{p+q-1}}{2}\right)^{-1}\left(u^{\prime}, u^{\prime \prime}\right) .
$$

We note that $\Psi^{-1}$ is the ordinary stereographic projection of the sphere $S^{p-1}$ if $q=1$.

We write $\left(\tilde{\Psi}^{*}\right)^{-1}={\widetilde{\left(\Psi^{-1}\right)}}^{*}$ for the twisted pull-back (in the sense of [47, Definition 2.3]) of the conformal map $\Psi^{-1}: M_{+} \rightarrow \mathbb{R}^{p+q-2}$, that is,

$$
\left(\tilde{\Psi}^{*}\right)^{-1}: C^{\infty}\left(\mathbb{R}^{p+q-2}\right) \rightarrow C^{\infty}\left(M_{+}\right)
$$

is given by

$$
\left(\tilde{\Psi}^{*}\right)^{-1}(F)(v):=\left(\frac{v_{0}+v_{p+q-1}}{2}\right)^{-\frac{p+q-4}{2}} F\left(\frac{2}{v_{0}+v_{p+q-1}}\left(\begin{array}{c}
v^{\prime} \\
v^{\prime \prime}
\end{array}\right)\right),
$$

where $v={ }^{t}\left(v_{0}, v^{\prime}, v^{\prime \prime}, v_{p+q-1}\right) \in M_{+}, v_{0}, v_{p+q-1} \in \mathbb{R}, v^{\prime} \in \mathbb{R}^{p-1}, v^{\prime \prime} \in \mathbb{R}^{q-1}$. In the group language (e.g. [41]), $\left(\widetilde{\Psi}^{*}\right)^{-1}$ is the standard intertwining operator from the $N$-picture to the $K$-picture. The map $\left(\widetilde{\Psi}^{*}\right)^{-1}$ will be applied also to other classes of functions.

Remark 2.1.2. Our manifold $M$ is a double cover of the generalized flag variety $G / \overline{P^{\max }}$ by a maximal parabolic subgroup $\overline{P^{\max }}$ (see $(2.3 .9)$ ). Then, $\left(\varpi^{p, q}, V^{p, q}\right)$ is identified with a subrepresentation of the degenerate principal series representation induced from a certain one-dimensional representation of $\overline{P^{\max }}$. In this framework, Fact [2.1.1] was proved by Kostant [50] for $p=q=4$ and by Binegar and Zierau [6], for general $p, q$ satisfying the condition that $p, q \geq 2$ and $p+q>4$. Zhu and Huang [80] identified this subrepresentation with the local theta correspondence associated to the dual pair $O(p, q) \times S L(2, \mathbb{R})$ in $S p(p+q, \mathbb{R})$ (to be more precise, its metaplectic cover) and the trivial one-dimensional representation of $S L(2, \mathbb{R})$.

Remark 2.1.3. If $p+q \geq 8$, then $\varpi^{p, q}$ becomes a minimal representation in the sense of Definition 1.5.1 (see [6]]).

\section{$2.2 \quad L^{2}$-model (the Schrödinger model)}

In Sections 2.2 and 2.3, we summarize the known results on the Schrödinger model of the minimal representation $\left(\pi, L^{2}(C)\right)$ of $G=O(p, q)$. The basic reference is [49]. 
A naive idea here is the following. Since $\Psi: \mathbb{R}^{p-1, q-1} \rightarrow M$ is a conformal map between two pseudo-Riemannian manifolds, we have

$$
\widetilde{\Psi}^{*}\left(\operatorname{Sol}\left(\widetilde{\Delta}_{M}\right)\right) \subset \operatorname{Sol}\left(\square_{\mathbb{R}^{p-1, q-1}}\right) .
$$

Since $M$ is compact, it follows from the formula (2.1.8) of $\widetilde{\Psi}^{*}$ that

$$
\widetilde{\Psi}^{*}\left(C^{\infty}(M)\right) \subset \mathcal{S}^{\prime}\left(\mathbb{R}^{p+q-2}\right),
$$

where $\mathcal{S}^{\prime}\left(\mathbb{R}^{p+q-2}\right)$ denotes the space of tempered distributions. By taking the Euclidean Fourier transform $\mathcal{F}_{\mathbb{R}^{p+q-2}}$, we get

$$
\left(\mathcal{F}_{\mathbb{R}^{p+q-2}} \circ \widetilde{\Psi}^{*}\right)\left(\operatorname{Sol}\left(\widetilde{\Delta}_{M}\right)\right) \subset\left\{u \in \mathcal{S}^{\prime}\left(\mathbb{R}^{p+q-2}\right): \operatorname{Supp} u \subset \bar{C}\right\} .
$$

It was proved in 49] that the right-hand side is contained in $L^{2}(C)$ (and in fact a dense subspace of $L^{2}(C)$ ). Let us fix some notation to formalize this fact.

We define a quadratic form by

$$
Q(x):=x_{1}^{2}+\cdots+x_{p-1}^{2}-x_{p}^{2}-\cdots-x_{p+q-2}^{2},
$$

which is the defining polynomial of the isotropic cone $C$ in $\mathbb{R}^{p+q-2}$.

The substitution $\delta(Q)$ of $Q$ into the Dirac delta function $\delta$ of one variable defines a distribution on $\mathbb{R}^{p+q-2} \backslash\{0\}$, which is represented as a measure, denoted by $d \mu$ on $C$. Alternatively, this measure is obtained as the volume form $\left.\alpha\right|_{C}$ where $\alpha$ is any $(p+q-3)$ form such that

$$
d Q \wedge \alpha=d x_{1} \wedge d x_{2} \wedge \cdots \wedge d x_{p+q-2}
$$

(see [24, Chapter III, Chapter 2]). An example of such $\alpha$ is given by

$$
\sum_{j=1}^{n} \frac{(-1)^{j-1} \epsilon_{j} x_{j} d x_{1} \wedge \cdots \wedge \widehat{d x}_{j} \wedge \cdots \wedge d x_{n}}{2\|x\|^{2}} .
$$

In the bipolar coordinate:

$$
\mathbb{R}_{+} \times S^{p-2} \times S^{q-2} \stackrel{\sim}{\rightarrow} C, \quad(r, \omega, \eta) \mapsto\left(\begin{array}{c}
r \omega \\
r \eta
\end{array}\right),
$$

the distribution $\delta(Q)$ is given by

$$
\langle\delta(Q), \varphi\rangle=\frac{1}{2} \int_{0}^{\infty} \int_{S^{p-2}} \int_{S^{q-2}} \varphi\left(\left(\begin{array}{c}
r \omega \\
r \eta
\end{array}\right)\right) r^{p+q-5} d r d \omega d \eta
$$


for a test function $\varphi$ on $\mathbb{R}^{p+q-2}$. Here, $d \omega$ and $d \eta$ denote the standard measures on $S^{p-2}$ and $S^{q-2}$, respectively. By this formula, we see that $\delta(Q)$ extends to a Schwartz distribution on $\mathbb{R}^{p+q-2}$ of measure class if $p+q>4$ because $r^{p+q-5} d r$ is locally integrable, then. Obviously, we have

$$
\operatorname{supp} \delta(Q)=C \cup\{0\} .
$$

Let $L^{2}(C) \equiv L^{2}(C, d \mu)$ be the Hilbert space consisting of square integrable functions on $C$. Thus, for a function $\varphi$ on $C$, the $L^{2}$-norm of $\varphi$ is given by

$$
\|\varphi\|_{L^{2}(C)}=\frac{1}{2} \int_{0}^{\infty} \int_{S^{p-2}} \int_{S^{q-2}}|\varphi(r \omega, r \eta)|^{2} r^{p+q-5} d r d \omega d \eta .
$$

Correspondingly to the coordinates, we have an isomorphism of Hilbert spaces:

$$
L^{2}\left(\mathbb{R}_{+}, \frac{1}{2} r^{p+q-5} d r\right) \widehat{\otimes} L^{2}\left(S^{p-2}\right) \widehat{\otimes} L^{2}\left(S^{q-2}\right) \simeq L^{2}(C) .
$$

Here, we employ the usual notation $\widehat{\otimes}$ for the Hilbert completion of the tensor product.

If $p+q>4$, then $u \mapsto u \delta(Q)$ defines a continuous, injective map from the Hilbert space $L^{2}(C)$ into the space $\mathcal{S}^{\prime}\left(\mathbb{R}^{p+q-2}\right)$ of tempered distributions on $\mathbb{R}^{p+q-2}$ :

$$
T: L^{2}(C) \rightarrow \mathcal{S}^{\prime}\left(\mathbb{R}^{p+q-2}\right), \quad u \mapsto u \delta(Q) .
$$

See [49, §3.4].

Now, we are ready to introduce a key map which will give an intertwining operator between the conformal model and the $L^{2}$-model.

$$
\mathcal{T}:=\left(\mathcal{F}_{\mathbb{R}^{p+q-2}} \circ \tilde{\Psi}^{*}\right)^{-1} \circ T=\left(\tilde{\Psi}^{*}\right)^{-1} \circ \mathcal{F}_{\mathbb{R}^{p+q-2}}^{-1} \circ T .
$$

For $u \in C_{0}^{\infty}(C), \mathcal{T} u \in C^{\infty}\left(M_{+}\right)$. We extend $\mathcal{T} u$ to a function on $M_{+} \cup$ $\left(-M_{+}\right)$by

$$
(\mathcal{T} u)(-v)=(-1)^{\frac{p-q}{2}}(\mathcal{T} u)(v) \quad\left(v \in M_{+}\right) .
$$

We recall from Fact 2.1 .1 that the inner product on $\overline{V^{p, q}}$ is given by the formula (2.1.7). Then, the main ingredient of [49, Theorem 4.9] can be restated as:

Fact 2.2.1. $\mathcal{T}$ extends to a unitary operator (up to scalar) from $L^{2}(C)$ onto $\overline{V^{p, q}}$. 
Remark 2.2.2. The definition (1.2.1) of the (Euclidean) Fourier transform $\mathcal{F}_{\mathbb{R}^{n}}$ adopted in this book involves the scalar multiplication by $(2 \pi)^{-\frac{n}{2}}$. Accordingly, the normalization of $\mathcal{T}$ is different from that of [49] by a scalar multiplication. In our normalization, we have

$$
\|\mathcal{T} u\|^{2}=\frac{1}{2}\|u\|_{L^{2}(C)}^{2} \quad\left(u \in L^{2}(C)\right)
$$

as we shall see in (3.1.11).

Through the unitary operator $\mathcal{T}$, we can transfer the unitary representation $\left(\varpi^{p, q}, \overline{V^{p, q}}\right)$ of $G=O(p, q)$ to a unitary representation, to be denoted by $\pi^{p, q}$, on the Hilbert space $L^{2}(C)$ by

$$
\pi^{p, q}(g):=\mathcal{T}^{-1} \circ \varpi^{p, q}(g) \circ \mathcal{T}, \quad g \in G .
$$

Hereafter, we shall write $\pi$ for $\pi^{p, q}$ for simplicity. Obviously, $\pi$ is irreducible because so is $\varpi^{p, q}$ (see Section 2.1). We note that the unitary inner product of $\pi$ is nothing but the $L^{2}$-inner product of $L^{2}(C)$. Naming after the classic Schrödinger model $L^{2}\left(\mathbb{R}^{n}\right)$ for the Weil representation of the metaplectic group (e.g. [18]), we shall say the resulting irreducible unitary representation $\left(\pi, L^{2}(C)\right)$ is the Schrödinger model for the minimal representation of $G=$ $O(p, q)$.

In the philosophy of the orbit method due to Kirillov and Kostant, the Schrödinger model may be regarded as a geometric quantization of the minimal nilpotent coadjoint orbit $\mathcal{O}_{\text {min }}$ in $\mathfrak{g}^{*}$, the dual of $\mathfrak{g}=\mathfrak{o}(p, q)$. We note that the isotropic cone $C$ is a Lagrangean variety of the symplectic manifold $\mathcal{O}_{\min }$.

So far, we have introduced two models of $\left(\varpi^{p, q}, \overline{V^{p, q}}\right)$ and $\left(\pi, L^{2}(C)\right)$ for the minimal representation of $G$. In the realization of $\overline{V^{p, q}}$, the $K$ structure is very clear to see, while on $L^{2}(C)$, its $K$-structure is not clear a priori. Generalizing the idea in [49] where we found explicitly an $L^{2}$-function (essentially, a $K$-Bessel function) on $C$ belonging to the minimal $K$-type, we shall explicitly find a formula of $K$-finite vectors of $L^{2}(C)$ in Chapter 3 . This computation is carried out by reducing the intertwining operator $\mathcal{T}$ to that of the Hankel transform of the $K$-Bessel functions.

\subsection{Lie algebra action on $L^{2}(C)$}

We continue the review of an easy part of the Schrödinger model $L^{2}(C)$ of $G=O(p, q)$ from Section 2.2. We shall explain how the Lie algebra $\mathfrak{g}=$ 
$\mathfrak{o}(p, q)$ acts on smooth vectors of $L^{2}(C)$. The action of a maximal parabolic subgroup $\overline{P^{\max }}$ on $L^{2}(C)$ will be also described. The main reference of this section is [49, §3].

Our notation here has the following relation with the notation in Introduction (Sections 1.1-1.3):

$$
\begin{aligned}
& p=n_{1}+1, q=n_{2}+1, \\
& n=n_{1}+n_{2}=p+q-2 .
\end{aligned}
$$

Let $e_{0}, \ldots, e_{p+q-1}$ be the standard basis of $\mathbb{R}^{p+q}$, on which $G=O(p, q)$ (see (1.7.1)) acts naturally. First, we define subgroups $M_{+}^{\max }, M^{\max }, K$, and $K^{\prime}$ of $G$ as follows:

$$
\begin{array}{rlrl}
m_{0} & :=-I_{p+q}, & \\
M_{+}^{\max } & :=\left\{g \in G: g \cdot e_{0}=e_{0}, g \cdot e_{p+q-1}=e_{p+q-1}\right\} & \simeq O(p-1, q-1), \\
M^{\max } & :=M_{+}^{\max } \cup m_{0} M_{+}^{\max } & \simeq O(p-1, q-1) \times \mathbb{Z}_{2}, \\
K & :=G \cap O(p+q) & \simeq O(p) \times O(q), \\
K^{\prime} & :=K \cap M_{+}^{\max } & & \simeq O(p-1) \times O(q-1) .
\end{array}
$$

Then, $K$ is a maximal compact subgroup of $G$ as we already used in Section 2.1, and $K^{\prime}$ is a maximal compact subgroup of $M_{+}^{\max }$. Corresponding to the maximal compact subgroup $K$, the Cartan involution $\theta$ of $G$ is given by $\theta(g)={ }^{t} g^{-1}$, and its differential (by the same notation) is given by $\theta(X)=$ $-{ }^{t} X$ in the matrix form.

We note that the group $M_{+}^{\max }$ acts on the isotropic cone $C$ in $\mathbb{R}^{p+q-2}$ transitively, and leaves the measure $d \mu$ (see Section 2.2) invariant.

Next we set

$$
\epsilon_{j}:= \begin{cases}1 & (1 \leq j \leq p-1), \\ -1 & (p \leq j \leq p+q-2) .\end{cases}
$$

Let $N_{j}, \bar{N}_{j}(1 \leq j \leq p+q-2)$ and $H$ be elements of the Lie algebra $\mathfrak{g}=\mathfrak{o}(p, q)$ given by

$$
\begin{aligned}
N_{j} & :=E_{j, 0}-E_{j, p+q-1}-\epsilon_{j} E_{0, j}-\epsilon_{j} E_{p+q-1, j}, \\
\bar{N}_{j} & :=E_{j, 0}+E_{j, p+q-1}-\epsilon_{j} E_{0, j}+\epsilon_{j} E_{p+q-1, j}, \\
H & :=E_{0, p+q-1}+E_{p+q-1,0} .
\end{aligned}
$$

We note that for $1 \leq j \leq p+q-2$,

$$
\begin{aligned}
\theta\left(N_{j}\right) & =\epsilon_{j} \bar{N}_{j}, \\
{\left[N_{j}, \bar{N}_{j}\right] } & =-2 \epsilon_{j} H .
\end{aligned}
$$


Then, we define abelian Lie algebras of $\mathfrak{g}$ by

$$
\begin{aligned}
\mathfrak{n}^{\max } & :=\sum_{j=1}^{p+q-2} \mathbb{R} N_{j}, \\
\overline{\mathfrak{n}^{\max }} & :=\sum_{j=1}^{p+q-2} \mathbb{R} \bar{N}_{j}, \\
\mathfrak{a} & :=\mathbb{R} H,
\end{aligned}
$$

and give coordinates for the corresponding abelian Lie subgroups $N^{\max }$, $\overline{N^{\max }}$ and $A$ by

$$
\begin{aligned}
& \mathbb{R}^{p+q-2} \simeq N^{\max }, a=\left(a_{1}, a_{2}, \cdots, a_{p+q-2}\right) \mapsto n_{a}:=\exp \left(\sum_{j=1}^{p+q-2} a_{j} N_{j}\right), \\
& \mathbb{R}^{p+q-2} \simeq \overline{N^{\max }}, \quad a=\left(a_{1}, a_{2}, \cdots, a_{p+q-2}\right) \mapsto \bar{n}_{a}:=\exp \left(\sum_{j=1}^{p+q-2} a_{j} \bar{N}_{j}\right), \\
& \mathbb{R} \simeq A, \quad t \mapsto \exp (t H) .
\end{aligned}
$$

Since $M_{+}^{\max }$ normalizes $N^{\max }$, we have a semidirect product group $M_{+}^{\max } N^{\max }$, which has the following matrix form:

$$
M_{+}^{\max } N^{\max }=\left\{g \in G: g\left(e_{0}+e_{p+q-1}\right)=e_{0}+e_{p+q-1}\right\} .
$$

On the other hand, the natural action of $G$ on $\mathbb{R}^{p+q}$ induces a transitive action on

$$
\widetilde{C}:=\left\{\left(x_{0}, \cdots, x_{p+q-1}\right) \in \mathbb{R}^{p+q} \backslash\{0\}: \sum_{j=0}^{p-1} x_{j}^{2}-\sum_{j=p}^{p+q-1} x_{j}^{2}=0\right\},
$$

with the isotropy subgroup $M_{+}^{\max } N^{\max }$ at $e_{0}+e_{p+q-1}$. Thus, we get a diffeomorphism

$$
G / M_{+}^{\max } N^{\max } \simeq \widetilde{C} .
$$

We define a maximal parabolic subgroup by

$$
\overline{P^{\max }}:=M^{\max } A \overline{N^{\max }} .
$$


Then the action of $\overline{P^{\max }}$ on $L^{2}(C)$ is described explicitly as follows ([49, $\S 3.3])$ :

$$
\begin{aligned}
(\pi(m) \psi)(x) & =\psi\left({ }^{t} m x\right) & & \left(m \in M_{+}^{\max }\right), \\
\left(\pi\left(m_{0}\right) \psi\right)(x) & =(-1)^{\frac{p-q}{2}} \psi(x), & & \\
\left(\pi\left(e^{t H}\right) \psi\right)(x) & =e^{-\frac{p+q-4}{2} t} \psi\left(e^{-t} x\right) & & (t \in \mathbb{R}), \\
\left(\pi\left(\bar{n}_{a}\right) \psi\right)(x) & =e^{2 \sqrt{-1}\left(a_{1} x_{1}+\cdots+a_{p+q-2} x_{p+q-2}\right)} \psi(x) & & \left(a \in \mathbb{R}^{p+q-2}\right) .
\end{aligned}
$$

For $g \notin \overline{P^{\max }}$, the action $\pi(g)$ on $L^{2}(C)$ was not given in [49]. (In fact, the formula $\pi(g)$ for general $g \in G$ is the main issue of the book.) Instead, we obtaind a formula for the differential action of the Lie algebra $\mathfrak{g}$ in [49]. To state the formula, we recall some general terminology for infinite dimensional representations adapted to our special setting.

Definition 2.3.1. Let $\psi \in L^{2}(C)$. We say $\psi$ is a differentiable vector if

$$
d \pi(X) \psi:=\lim _{t \rightarrow 0} \frac{\pi\left(e^{t X}\right) \psi-\psi}{t}
$$

exists for any $X \in \mathfrak{g}$. Iterating this process, we say $\psi$ is a smooth vector if $d \pi\left(X_{1}\right) \cdots d \pi\left(X_{k}\right) \psi$ is a differentiable vector for any $k \geq 1$ and $X_{1}, \ldots, X_{k} \in$ $\mathfrak{g}$. (The notion of smooth vectors is defined for continuous representations on complete, locally convex topological vector spaces.)

We say $\psi$ is $K$-finite if

$$
\operatorname{dim}_{\mathbb{C}} \mathbb{C}-\operatorname{span}\{\pi(k) \psi: k \in K\}<\infty .
$$

Let $L^{2}(C)^{\infty}$ be the space of smooth vectors of the unitary representation $\left(\pi, L^{2}(C)\right)$ of $G$, and $L^{2}(C)_{K}$ the space of $K$-finite vectors of $L^{2}(C)$. Applying the general theory of representations of real reductive Lie groups (see [41, 78, for example) to our irreducible unitary representation $\left(\pi, L^{2}(C)\right)$, we have

1) $L^{2}(C)^{\infty}$ has naturally a Fréchet topology, on which both the group $G$ and the Lie algebra $\mathfrak{g}$ act continuously.

2) $L^{2}(C)_{K} \subset L^{2}(C)^{\infty} \subset L^{2}(C)$. Moreover, $L^{2}(C)_{K}$ is dense in $L^{2}(C)^{\infty}$, and $L^{2}(C)^{\infty}$ is dense in $L^{2}(C)$, in each topology. 
From now, we shall use the notation which is compatible with the notation of Chapter 1:

$$
n=n_{1}+n_{2}=(p-1)+(q-1) .
$$

It follows from (2.3.12) and (2.3.13) that the differential action of $A$ and $\overline{N^{\max }}$ on $L^{2}(C)^{\infty}$ is given as

$$
\begin{aligned}
& d \pi(H)=-\left(E+\frac{n-2}{2}\right), \\
& d \pi\left(\bar{N}_{j}\right)=2 \sqrt{-1} x_{j} \quad(1 \leq j \leq n) .
\end{aligned}
$$

Here, $E:=\sum_{i=1}^{n} x_{i} \frac{\partial}{\partial x_{i}}$ is the Euler operator, and $x_{j}$ in (2.3.15) is the multiplication operator by the coordinate function $x_{j}$.

On the other hand, the differential action of $N^{\max }$ on $L^{2}(C)^{\infty}$ is more complicated. In [49, Lemma 3.2], we gave its formula by means of second order differential operators in the ambient space $\mathbb{R}^{n}=\mathbb{R}^{p+q-2}$ via the inclusion map $T: L^{2}(C) \hookrightarrow \mathcal{S}^{\prime}\left(\mathbb{R}^{n}\right), u \mapsto u \delta(Q)$ (see (2.2.6)) as follows. Let $D_{j}$ be a differential operator on $\mathbb{R}^{n}$ (in the notation loc. cit., $D_{j}=d \hat{\varpi}_{\lambda, \epsilon}\left(N_{j}\right)$ with $\lambda=\frac{p+q-4}{2}$ ) given by:

$$
D_{j}=\sqrt{-1}\left(-\frac{n-2}{2} \epsilon_{j} \frac{\partial}{\partial x_{j}}-\left(\sum_{k=1}^{n} x_{k} \frac{\partial}{\partial x_{k}}\right) \epsilon_{j} \frac{\partial}{\partial x_{j}}+\frac{1}{2} x_{j}\left(\sum_{k=1}^{n} \epsilon_{k} \frac{\partial^{2}}{\partial x_{k}^{2}}\right)\right) .
$$

Then the differential action $d \pi\left(N_{j}\right)(1 \leq j \leq n)$ is characterized by the commutative diagram:

$$
\begin{array}{ccc}
L^{2}(C)^{\infty} & \stackrel{T}{\longrightarrow} & \mathcal{S}^{\prime}\left(\mathbb{R}^{n}\right) \\
d \pi\left(N_{j}\right) \downarrow & & \\
L^{2}(C)^{\infty} & \stackrel{T}{\longrightarrow} D_{j} & \mathcal{S}^{\prime}\left(\mathbb{R}^{n}\right) .
\end{array}
$$

In Section 2.4, we shall treat these differential operators more systematically by introducing the following differential operators $P_{j}(b)(1 \leq j \leq n)$ with complex parameter $b$ by

$$
P_{j}(b):=\epsilon_{j} x_{j} \square-(2 E+n-2 b) \frac{\partial}{\partial x_{j}},
$$

where we set $\square=\sum_{j=1}^{n} \epsilon_{j} \frac{\partial^{2}}{\partial x_{j}^{2}}$. By definition, we have

$$
D_{j}=\frac{\sqrt{-1}}{2} \epsilon_{j} P_{j}(-1) \quad(1 \leq j \leq n) .
$$


In Theorem 2.4.1, we shall see that the differential operator $P_{j}:=P_{j}(1)$ is tangential to $C$, and that the differential action $d \pi\left(N_{j}\right)$ is given as

$$
d \pi\left(N_{j}\right)=\left.\frac{\sqrt{-1}}{2} \epsilon_{j} P_{j}\right|_{C} \quad(1 \leq j \leq n) .
$$

\subsection{Commuting differential operators on $C$}

In this section, we investigate basic properties of the differential operators

$$
P_{j}(b)=\epsilon_{j} x_{j} \square-(2 E+n-2 b) \frac{\partial}{\partial x_{j}} \quad(1 \leq j \leq n)
$$

on $\mathbb{R}^{n}$ introduced in (2.3.18), and in particular, explain why and how the differential operators $P_{j}(-1)(1 \leq j \leq n)$ (see (2.3.18) $)$ induce the differential operators $P_{j}(1)$ along the isotropic cone $C$.

Again we recall

$$
n=n_{1}+n_{2}=(p-1)+(q-1) .
$$

We also recall from Section 1.1 that $\mathbb{R}\left[x, \frac{\partial}{\partial x}\right] \equiv \mathbb{R}\left[x_{1}, \ldots, x_{n}, \frac{\partial}{\partial x_{1}}, \ldots, \frac{\partial}{\partial x_{n}}\right]$ is the Weyl algebra and that $\mathbb{R}\left[x, \frac{\partial}{\partial x}\right]^{C}$ is the subalgebra consisting of differential operators tangential to $C$.

The main result of this section is the following theorem concerning with the fundamental differential operators on the isotropic cone defined by

$$
P_{j}:=P_{j}(1) \quad(1 \leq j \leq n) .
$$

Theorem 2.4.1. 1) $P_{i} P_{j}=P_{j} P_{i}(1 \leq i, j \leq n)$.

2) $P_{j} \in \mathbb{R}\left[x, \frac{\partial}{\partial x}\right]^{C}(1 \leq j \leq n)$.

3) The Lie algebra generated by

$$
\left[P_{i}, x_{j}\right]=P_{i} x_{j}-x_{j} P_{i} \quad(1 \leq i, j \leq n)
$$

in the Weyl algebra $\mathbb{R}\left[x, \frac{\partial}{\partial x}\right]$ is isomorphic to $\mathfrak{o}(p-1, q-1)+\mathbb{R}$, the Lie algebra of the differential action of the conformal linear transformation group $C O(Q)$.

4) $\left.P_{j}\right|_{C}$ extends to a self-adjoint operator on $L^{2}(C)$.

5) $\left.\left(\sum_{j=1}^{n} \epsilon_{j} P_{j}^{2}\right)\right|_{C}=0$. 
We shall give a proof of Theorem 2.4.1 in the following order: (2), (4), (5), (1), and (3). An important step for the proof is the following:

Proposition 2.4.2. For $u \in C_{0}^{\infty}\left(\mathbb{R}^{n} \backslash\{0\}\right)$, we have:

1) $P_{j}(-1)(u \delta(Q))=\left(P_{j}(1) u\right) \delta(Q)$.

Hence, we have $P_{j}(-1) \circ T=T \circ P_{j}(1)$.

2) $P_{j}(1)(u Q)=\left(P_{j}(-1) u\right) Q$.

In particular, $\left.P_{j}(1)(u Q)\right|_{C}=0$.

Admitting Proposition 2.4.2 for a while, we give a proof of Theorem 2.4.1 (2) and (4).

Proof of Theorem 2.4.1 (2). For a smooth function $\psi$ defined in an open subset $V$ of $C$, we extend it to a smooth function $\tilde{\psi}$ in an open subset of $\mathbb{R}^{n} \backslash\{0\}$. Then, $\left.P_{j}(1) \tilde{\psi}\right|_{V}$ is independent of the choice of the extension $\tilde{\psi}$, and is determined by $\psi=\left.\tilde{\psi}\right|_{V}$. In fact, let $\tilde{\psi}_{1}, \tilde{\psi}_{2}$ be extensions of $\psi$. Since $\left.\left(\tilde{\psi}_{1}-\tilde{\psi}_{2}\right)\right|_{C}=0$, we find a smooth function locally defined in a neighborhood of $V$ such that

$$
\tilde{\psi}_{1}-\tilde{\psi}_{2}=u Q
$$

Then $\left.\left(P_{j} \tilde{\psi}_{1}-P_{j} \tilde{\psi}_{2}\right)\right|_{C}=\left.\left(P_{j}(-1) u\right) Q\right|_{C}=0$ by Proposition 2.4.2 (2). Therefore, $\left.P_{j} \psi_{1}\right|_{C}=\left.P_{j} \psi_{2}\right|_{C}$. Thus, we have a well-defined map

$$
C^{\infty}(V) \rightarrow C^{\infty}(V),\left.\quad \psi \mapsto P_{j}(1) \tilde{\psi}\right|_{V}
$$

Since this is a sheaf morphism, it is given by a differential operator on $C$. Hence, $P_{j}=P_{j}(1)$ is tangential to $C$. Therefore, Theorem 2.4.1 (2) is shown.

By Proposition 2.4.2, we also get the self-adjointness of $\left.P_{j}(1)\right|_{C}$ as follows.

Proof of Theorem 2.4.1 (4). By (2.3.17), the differential action $d \pi\left(N_{j}\right)$ is characterized by the relation

$$
D_{j} \circ T=T \circ d \pi\left(N_{j}\right) .
$$

On the other hand, we have proved in Proposition 2.4.2 (1)

$$
P_{j}(-1) \circ T=T \circ\left(\left.P_{j}(1)\right|_{C}\right) .
$$


Since $D_{j}=\frac{\sqrt{-1}}{2} \epsilon_{j} P_{j}(-1)$ by definition, we get

$$
d \pi\left(N_{j}\right)=\left.\frac{\sqrt{-1}}{2} \epsilon_{j} P_{j}(1)\right|_{C}
$$

As $\left(\pi, L^{2}(C)\right)$ is a unitary representation of the Lie group $G=O(p, q)$, the differential action $\sqrt{-1} d \pi(X)$ on $L^{2}(C)^{\infty}$ extends to a self-adjoint operator on $L^{2}(C)$ for any $X \in \mathfrak{g}=\mathfrak{o}(p, q)$. Hence, $P_{j}=P_{j}(1)$ is self-adjoint. Thus, Theorem 2.4.1 (4) is proved.

Before giving a proof of Proposition 2.4.2, we set up the notation of the meromorphic continuation of the distribution $Q(x)_{+}^{\lambda}$. For a complex parameter $\lambda$ with $\operatorname{Re} \lambda>-1$, we define a distribution on $\mathbb{R}^{n}$ by

$$
Q(x)_{+}^{\lambda}:= \begin{cases}Q(x)^{\lambda} & \text { if } Q(x)>0 \\ 0 & \text { if } Q(x) \leq 0\end{cases}
$$

Then, $Q(x)_{+}^{\lambda}$ continues as a distribution depending meromorphically on the parameter $\lambda \in \mathbb{C}$ (see Appendix 7.1). In particular, as a distribution on $\mathbb{R}^{n} \backslash\{0\}, Q(x)_{+}^{\lambda}$ has only simple poles located at $\lambda=-1,-2,-3, \ldots$ Since the gamma function $\Gamma(\lambda+1)$ has simple poles exactly at the same places $\lambda=$ $-1,-2, \ldots$, we see that $\frac{Q(x)_{+}^{\lambda}}{\Gamma(\lambda+1)}$ defines a distribution on $\mathbb{R}^{n} \backslash\{0\}$ depending holomorphically on $\lambda \in \mathbb{C}$.

In view of the residue formula (7.1.1) in Appendix 7.1 for the one variable case $\frac{t_{+}^{\lambda}}{\Gamma(\lambda+1)}$, we have the following formula of generalized functions on $\mathbb{R}^{n} \backslash$ $\{0\}$ (see [24, Chapter III, $\S 2.2]$ ):

$$
\delta(Q(x))=\left.\frac{Q(x)_{+}^{\lambda}}{\Gamma(\lambda+1)}\right|_{\lambda=-1} .
$$

Therefore, the map $T: L^{2}(C) \rightarrow \mathcal{S}^{\prime}\left(\mathbb{R}^{n}\right)$ defined in (2.2.6) has the following expression:

$$
T\left(\left.u\right|_{C}\right)=\left.\frac{u Q(x)_{+}^{\lambda}}{\Gamma(\lambda+1)}\right|_{\lambda=-1}
$$

for $u \in C_{0}^{\infty}\left(\mathbb{R}^{n} \backslash\{0\}\right)$. The proof of Proposition 2.4.2 will make use of (2.4.2). Along this line, we prepare:

Lemma 2.4.3. For $u \in C_{0}^{\infty}\left(\mathbb{R}^{n} \backslash\{0\}\right)$,

$$
\square\left(u Q_{+}^{\lambda}\right)=(\square u) Q_{+}^{\lambda}+4 \lambda(E u) Q_{+}^{\lambda-1}+2 \lambda(2 \lambda+n-2) u Q_{+}^{\lambda-1} .
$$


Similar formulas also hold if we replace $Q_{+}$by $Q$ for positive integers $\lambda$. In particular, letting $\lambda=1$, we have

$$
\square(u Q)=(\square u) Q+(4 E+2 n) u .
$$

Proof. By the Leibniz rule, we have

$$
\begin{aligned}
\frac{\partial}{\partial x_{j}}\left(u Q_{+}^{\lambda}\right)= & \frac{\partial u}{\partial x_{j}} Q_{+}^{\lambda}+2 \lambda \epsilon_{j} x_{j} u Q_{+}^{\lambda-1}, \\
\frac{\partial^{2}}{\partial x_{j}}\left(u Q_{+}^{\lambda}\right)= & \frac{\partial^{2} u}{\partial x_{j}^{2}} Q_{+}^{\lambda}+4 \lambda \epsilon_{j} x_{j} \frac{\partial u}{\partial x_{j}} Q_{+}^{\lambda-1} \\
& +2 \lambda \epsilon_{j} u Q_{+}^{\lambda-1}+4 \lambda(\lambda-1) x_{j}^{2} u Q_{+}^{\lambda-2} .
\end{aligned}
$$

Summing up (2.4.4) multiplied by the signature $\epsilon_{j}$ over $j$, we get Lemma.

Here is a key formula for the proof of Proposition 2.4.2:

Lemma 2.4.4. For $u \in C_{0}^{\infty}\left(\mathbb{R}^{n} \backslash\{0\}\right)$, we have the following identity as distributions on $\mathbb{R}^{n} \backslash\{0\}$ :

$$
P_{j}(b)\left(u Q_{+}^{\lambda}\right)=\left(P_{j}(b-2 \lambda) u\right) Q_{+}^{\lambda}-4 \lambda(\lambda-b) \epsilon_{j} x_{j} u Q_{+}^{\lambda-1}
$$

for any $1 \leq j \leq n, b \in \mathbb{C}$, and $\lambda \in \mathbb{C} \backslash\{-1,-2, \ldots\}$.

Proof. By (2.4.3), we have

$$
\begin{aligned}
(2 E+n-2 b) \frac{\partial}{\partial x_{j}}\left(u Q_{+}^{\lambda}\right)= & \left.(2 E+n-2(b-2 \lambda)) \frac{\partial u}{\partial x_{j}}\right) Q_{+}^{\lambda} \\
& +2 \lambda(4 \lambda+n-2-2 b) \epsilon_{j} x_{j} u Q_{+}^{\lambda-1} \\
& +4 \lambda \epsilon_{j} x_{j}(E u) Q_{+}^{\lambda-1} .
\end{aligned}
$$

Then, (2.4.5) follows from Lemma 2.4.3.

Now we are ready to prove Proposition 2.4.2.

Proof of Proposition 2.4.2. Since $\frac{Q_{+}^{\lambda}}{\Gamma(\lambda+1)}$ is a distribution on $\mathbb{R}^{n} \backslash\{0\}$ with parameter holomorphically dependent on $\lambda \in \mathbb{C}$, we have

$$
P_{j}(b)\left(u \frac{Q_{+}^{\lambda}}{\Gamma(\lambda+1)}\right)=\left(P_{j}(b-2 \lambda) u\right) \frac{Q_{+}^{\lambda}}{\Gamma(\lambda+1)}-(\lambda-b) \epsilon_{j} x_{j} u \frac{Q_{+}^{\lambda-1}}{\Gamma(\lambda)}
$$

for all $\lambda \in \mathbb{C}$. By letting $b=\lambda$, we have

$$
P_{j}(\lambda)\left(u \frac{Q_{+}^{\lambda}}{\Gamma(\lambda+1)}\right)=\left(P_{j}(-\lambda) u\right) \frac{Q_{+}^{\lambda}}{\Gamma(\lambda+1)} .
$$


Further, by letting $\lambda=-1$, we get

$$
P_{j}(-1)(u \delta(Q))=\left(P_{j}(1) u\right) \delta(Q) .
$$

By letting $\lambda=1$, we get

$$
P_{j}(1)(u Q)=\left(P_{j}(-1) u\right) Q
$$

Therefore, we have proved Proposition 2.4.2.

Thus, the proof of Theorem 2.4.1 (2) is completed. Next, let us prove the following:

Proposition 2.4.5. $\sum_{j=1}^{n} \epsilon_{j} P_{j}(1)^{2}=Q \square^{2}$.

Admitting Proposition 2.4.5 for the time being, we give a proof of Theorem 2.4.1 (5).

Proof of Theorem 2.4 .1 (5). We have already shown that $P_{j}(1) \in \mathbb{R}\left[x, \frac{\partial}{\partial x}\right]^{C}$ $(1 \leq j \leq n)$. Then, Theorem 2.4.1 (5) is an immediate consequence of the following identity in the algebra $\mathbb{R}\left[x, \frac{\partial}{\partial x}\right]^{C}$.

To prove Proposition 2.4.5, we list some basic relations of the Lie bracket $[A, B]=A B-B A$ in the Weyl algebra $\mathbb{R}\left[x, \frac{\partial}{\partial x}\right]$ :

\section{Claim 2.4.6.}
1) $\left[E, x_{j}\right]=x_{j}$.
2) $\left[E, \frac{\partial}{\partial x_{j}}\right]=-\frac{\partial}{\partial x_{j}}$.
3) $[E, \square]=-2 \square$.
4) $\left[\square, x_{j}\right]=2 \epsilon_{j} \frac{\partial}{\partial x_{j}}$.

Here, $x_{j}$ denotes the multiplication operator by $x_{j}$.

Proof. Straightforward by the Leibniz rule. 
Proof of Proposition 2.4.5. In light of the definition

$$
P_{i}(b)=\epsilon_{i} x_{i} \square-(2 E+n-2 b) \frac{\partial}{\partial x_{i}},
$$

we develop $P_{i}(b) P_{j}(b)$ as

$$
P_{i}(b) P_{j}(b)=(\mathrm{I})+(\mathrm{II})+(\mathrm{III})+(\mathrm{IV}),
$$

where $(\mathrm{I}) \equiv(\mathrm{I})_{i j}(b)$ is given by

$$
\begin{aligned}
(\mathrm{I}) & :=\left(\epsilon_{i} x_{i} \square\right)\left(\epsilon_{j} x_{j} \square\right) \\
& =\epsilon_{i} \epsilon_{j} x_{i} x_{j} \square^{2}+2 \epsilon_{i} x_{i} \frac{\partial}{\partial x_{j}} \square,
\end{aligned}
$$

and similarly,

$$
\begin{aligned}
(\mathrm{II}) & :=-\left(\epsilon_{i} x_{i} \square\right)\left((2 E+n-2 b) \frac{\partial}{\partial x_{j}}\right) \\
& =-\epsilon_{i}(2 E+n-2 b+2) x_{i} \frac{\partial}{\partial x_{j}} \square, \\
(\mathrm{III}) & :=-\left((2 E+n-2 b) \frac{\partial}{\partial x_{i}}\right)\left(\epsilon_{j} x_{j} \square\right) \\
& =-\epsilon_{j}(2 E+n-2 b)\left(x_{j} \frac{\partial}{\partial x_{i}}+\delta_{i j}\right) \square, \\
(\mathrm{IV}) & :=\left((2 E+n-2 b) \frac{\partial}{\partial x_{i}}\right)\left((2 E+n-2 b) \frac{\partial}{\partial x_{j}}\right) \\
& =\left(4 E^{2}+4(n+1-2 b) E+(n-2 b)(n+2-2 b)\right) \frac{\partial^{2}}{\partial x_{i} \partial x_{j}} .
\end{aligned}
$$

Here, $\delta_{i j}$ stands for Kronecker's delta. Now, we take $i=j$ and $b=1$, and sum up these terms over $j$ :

$$
\begin{aligned}
& \sum_{j=1}^{n} \epsilon_{j}(\mathrm{I})_{j j}(1)=Q \square^{2}+2 E \square, \\
& \sum_{j=1}^{n} \epsilon_{j}(\mathrm{II})_{j j}(1)=-(2 E+n) E \square \\
& \sum_{j=1}^{n} \epsilon_{j}(\mathrm{III})_{j j}(1)=-(2 E+n-2)(E+n) \square, \\
& \sum_{j=1}^{n} \epsilon_{j}(\mathrm{IV})_{j j}(1)=\left(4 E^{2}+4(n-1) E+(n-2) n\right) \square .
\end{aligned}
$$


Summing up these four equations, we get Proposition 2.4.5.

It is easy to see that the formulas for (I), (II), (III), and (IV) used in the previous proof lead us also to the following:

Proposition 2.4.7. As differential operators on $\mathbb{R}^{n}$, we have

$$
\left[P_{i}(b), P_{j}(b)\right]=0
$$

for any $1 \leq i, j \leq n$ and $b \in \mathbb{C}$.

As a special case of Proposition 2.4.7, we have:

Proof of Theorem 2.4.1 (1). This follows from Proposition 2.4.7 by letting $b=1$.

Finally, let us prove Theorem 2.4.1 (3).

Proof of Theorem 2.4.1 (3). We continue the notation (2.3.1) for $\epsilon_{j}= \pm 1$ $(1 \leq j \leq n=p+q-2)$.

Let $E_{i j}(1 \leq i, j \leq n)$ be the matrix unit. Then

$$
\epsilon_{i} \epsilon_{j} E_{i j}-E_{j i} \quad(1 \leq i<j \leq n)
$$

forms a basis of the Lie algebra $\mathfrak{o}(p-1, q-1)$ of the Lie group $M_{+}^{\max } \simeq$ $O(p-1, q-1)$. Correspondingly, the natural differential action on $\mathbb{R}^{n}=$ $\mathbb{R}^{(p-1)+(q-1)}$ gives a vector field

$$
X_{i j}:=\epsilon_{i} \epsilon_{j} x_{i} \frac{\partial}{\partial x_{j}}-x_{j} \frac{\partial}{\partial x_{i}} \quad(1 \leq i<j \leq n) .
$$

Likewise, the differential of the dilation is given by the Euler vector field

$$
E=\sum_{i=1}^{n} x_{i} \frac{\partial}{\partial x_{i}} .
$$

Hence, Theorem 2.4.1 (3) is an immediate consequence of the following lemma.

Lemma 2.4.8. As differential operators on $\mathbb{R}^{n}$, we have
1) $\left[P_{i}, x_{j}\right]=X_{i j} \quad(1 \leq i<j \leq n)$.
2) $\left[P_{i}, x_{i}\right]=-2(E+n-2) \quad(1 \leq i \leq n)$. 
Proof. By the definition (2.3.18) of $P_{j}=P_{j}(1)$, we have

$$
\begin{aligned}
{\left[P_{i}, x_{j}\right]=} & \epsilon_{i} x_{i}\left[\square, x_{j}\right]-(2 E+n-2)\left[\frac{\partial}{\partial x_{i}}, x_{j}\right] \\
- & {\left[2 E+n-2, x_{j}\right] \frac{\partial}{\partial x_{i}} . }
\end{aligned}
$$

By Claim 2.4.6, we have

$$
\begin{aligned}
& =2 \epsilon_{i} \epsilon_{j} x_{i} \frac{\partial}{\partial x_{j}}-\delta_{i j}(2 E+n-2)-2 x_{j} \frac{\partial}{\partial x_{i}} \\
& =2 X_{i j}-\delta_{i j}(2 E+n-2) .
\end{aligned}
$$

Hence, Lemma is proved.

Hence, the proof of Theorem 2.4.1 is completed.

Remark 2.4.9. Let $R$ be the subalgebra of $\mathbb{R}\left[x, \frac{\partial}{\partial x}\right]^{C}$ generated by $x_{k}, E$, and $X_{i j}(1 \leq k \leq n, 1 \leq i<j \leq n)$. Then, $P_{j} \in \mathbb{R}\left[x, \frac{\partial}{\partial x}\right]^{C}$ but $P_{j} \notin R$.

To see this, we say an element $P$ of the Weyl algebra $\mathbb{R}\left[x, \frac{\partial}{\partial x}\right]$ is of degree $k$ if

$$
[E, P]=k P \text {. }
$$

If $P, Q$ are of degree $k, l$, respectively, then $P Q$ is of degree $k+l$ because

$$
[E, P Q]=[E, P] Q+P[E, Q] .
$$

Since $x_{k}$ is of degree 1 , and $E$ and $X_{i j}$ is of degree 0 , any element of $R$ is expressed as a linear combination of operators of non-negative degrees. Since $P_{j}$ is of degree -1 , we conclude $P_{j} \notin R$.

Remark 2.4.10. Our concern here is with the case $n>2($ i.e. $p+q>4)$. Let us examine the degenerate case where $n=2$ and $n_{1}=n_{2}=1$ (i.e. $p=q=2)$. Then, the polynomial $Q(x)=x_{1}^{2}-x_{2}^{2}$ is not irreducible, and the differential operators $P_{1}$ and $P_{2}$ have the following formulas:

$$
\begin{aligned}
& P_{1}+P_{2}=-\left(x_{1}+x_{2}\right)\left(\frac{\partial}{\partial x_{1}}+\frac{\partial}{\partial x_{2}}\right)^{2}, \\
& P_{1}-P_{2}=-\left(x_{1}-x_{2}\right)\left(\frac{\partial}{\partial x_{1}}-\frac{\partial}{\partial x_{2}}\right)^{2} .
\end{aligned}
$$

By the change of variables

$$
y_{1}=x_{1}+x_{2}, \quad y_{2}=x_{1}-x_{2},
$$


the isotropic cone $C$ is given by

$$
\left\{\left(y_{1}, y_{2}\right) \in \mathbb{R}^{2} \backslash\{0\}: y_{1}=0 \text { or } y_{2}=0\right\},
$$

and we have

$$
\begin{aligned}
& P_{1}+P_{2}=-4 y_{1} \frac{\partial^{2}}{\partial y_{1}^{2}}, \\
& P_{1}-P_{2}=-4 y_{2} \frac{\partial^{2}}{\partial y_{2}^{2}} .
\end{aligned}
$$

\subsection{The unitary inversion operator $\mathcal{F}_{C}=\pi\left(w_{0}\right)$}

In this section, we list some important features of the element

$$
w_{0}=\left(\begin{array}{cc}
I_{p} & 0 \\
0 & -I_{q}
\end{array}\right) \in G,
$$

and investigate key properties of the corresponding unitary operator

$$
\mathcal{F}_{C}:=\pi\left(w_{0}\right)
$$

on $L^{2}(C)$.

I0 (Order two) Obviously, the element $w_{0}$ is of order two. Therefore, $\mathcal{F}_{C}^{2}=$ id on $L^{2}(C)$.

I1 (Cartan involution) The Cartan involution $\theta(g)={ }^{t} g^{-1}$ is given as the conjugation by $w_{0}$ :

$$
\theta(g)=w_{0} g w_{0}^{-1}
$$

because ${ }^{t} g w_{0} g=w_{0}$ for $g \in G$.

I2 (Center of $K$ ) $w_{0}$ lies in the center of $K$. This is obvious from (2.5.1). It also follows directly from the definition of $w_{0}$ in the matrix form.

I3 (Bruhat decomposition) Retain the notation as in Section 2.3. Then,

$$
\operatorname{Ad}\left(w_{0}\right) H=-H
$$

and therefore $\left.\operatorname{Ad}\left(w_{0}\right)\right|_{\mathfrak{a}}=-\mathrm{id}$. We also see readily from (2.3.5) and (2.5.1) that

$$
\operatorname{Ad}\left(w_{0}\right) \bar{N}_{j}=\epsilon_{j} N_{j} \quad(1 \leq j \leq p+q-2)
$$


and therefore

$$
\operatorname{Ad}\left(w_{0}\right) \mathfrak{n}^{\max }=\overline{\mathfrak{n}^{\max }} .
$$

The Gelfand-Naimark decomposition

$$
\mathfrak{g}=\left(\mathfrak{m}^{\max }+\mathfrak{a}+\overline{\mathfrak{n}^{\max }}\right)+\mathfrak{n}^{\max }=\overline{\mathfrak{p}^{\max }}+\operatorname{Ad}\left(w_{0}\right) \overline{\mathfrak{n}^{\max }}
$$

gives, in turn, the following Bruhat decomposition of $G$ on the group level:

$$
G=\overline{P^{\max }} w_{0} \overline{P^{\max }} \amalg \overline{P^{\max }} .
$$

I4 (Restricted root system) Let $\mathfrak{g}=\mathfrak{k}+\mathfrak{p}$ be the Cartan decomposition, and we take a maximal abelian subalgebra $\mathfrak{b}$ of $\mathfrak{p}$. Since $\operatorname{Ad}\left(w_{0}\right)$ acts on $\mathfrak{p}$ as $-\mathrm{id}, w_{0}$ acts on the restricted root system $\Sigma(\mathfrak{g}, \mathfrak{b})$ as $-\mathrm{id}$. We note that the longest element in the Weyl group $W(\Sigma(\mathfrak{g}, \mathfrak{b}))$ is equal to - id if $p \neq q$ or if $p=q \in 2 \mathbb{Z}$. In the case $p=q \in 2 \mathbb{Z}+1$, the restricted root system $\Sigma(\mathfrak{g}, \mathfrak{b})$ is of type $D_{q}$ and

$$
-\operatorname{id} \notin W(\Sigma(\mathfrak{g}, \mathfrak{b})) \simeq \mathfrak{S}_{q} \ltimes(\mathbb{Z} / 2 \mathbb{Z})^{q-1},
$$

and therefore $\left.\operatorname{Ad}\left(w_{0}\right)\right|_{\mathfrak{b}}=-$ id gives an outer automorphism on $\Sigma(\mathfrak{g}, \mathfrak{b})$.

I5 (Jordan algebras) We regard $\mathbb{R}^{p-1, q-1} \simeq \mathbb{R}^{p+q-2}$ as the semisimple Jordan algebra with indefinite quadratic form $Q(x)$ (see (1.1.1)). This Jordan algebra is euclidean if $\min (p, q)=2$, and non-euclidean if $p, q>2$. The conformal group (Kantor-Koecher-Tits group) of $\mathbb{R}^{p-1, q-1}$ is equal to the group $G=O(p, q)$, and the action of the element $w_{0}$ on $\mathbb{R}^{p-1, q-1}$ corresponds to the conformal inversion $x \mapsto-x^{-1}$ (see [16, 65]). Thus, we call $w_{0}$ the conformal inversion element. The structure group

$$
L_{+}:=M_{+}^{\max } A \simeq C O(Q)=O(p-1, q-1) \times \mathbb{R}_{>0}
$$

acts on $\mathbb{R}^{p-1, q-1}$ by $x \mapsto e^{t} m x$ for $\left(m, e^{t H}\right) \in M_{+}^{\max } \times A$, and on its dual space by $x \mapsto e^{-t t} m^{-1} x$.

I6 (The action on the minimal representation) In the conformal model $\left(\varpi^{p, q},{\overline{V^{p, q}}}\right.$ ) (see Section 2.1), the whole group $G$ acts on the geometry, and therefore, the unitary operator $\varpi^{p, q}\left(w_{0}\right)$ is easy to describe:

$$
\left(\varpi^{p, q}\left(w_{0}\right) h\right)\left(v_{0}, \ldots, v_{p+q-1}\right)=h\left(v_{0}, \ldots, v_{p-1},-v_{p}, \ldots,-v_{p+q-1}\right),
$$

(see the definition (2.1.5)). Then, by using the intertwining operator $\mathcal{T}: L^{2}(C) \rightarrow \overline{V^{p, q}}$ (see $(2.2 .7)$ ), we get the formula of $\mathcal{F}_{C}=\pi\left(w_{0}\right)$ in the Schrödinger model $\left(\pi, L^{2}(C)\right)$ as

$$
\mathcal{F}_{C}=\mathcal{T}^{-1} \circ \varpi^{p, q}\left(w_{0}\right) \circ \mathcal{T} .
$$


However, we do not know how to find the formulas (1.3.1)-(1.3.3) of $\mathcal{F}_{C}$ directly from (2.5.7) and the definition of $\mathcal{T}$. Thus, in order to find the unitary inversion formulas of $\mathcal{F}_{C}=\pi\left(w_{0}\right)$, we shall take a roundabout course (by using the $K$-type decomposition in part) in later chapters.

Now, let us study basic properties of the unitary inversion operator $\mathcal{F}_{C}=$ $\pi\left(w_{0}\right)$ on $L^{2}(C)$. First, we extend $\mathcal{F}_{C}$ to distribution vectors.

Let $L^{2}(C)^{-\infty}$ be the continuous dual of the Fréchet space $L^{2}(C)^{\infty}$ (Definition 2.3.1), namely, the space of continuous $\mathbb{C}$-linear maps $L^{\infty}(C) \rightarrow \mathbb{C}$. Elements in $L^{2}(C)^{-\infty}$ are called distribution vectors.

We introduce a conjugate linear map

$$
\iota: L^{2}(C) \rightarrow L^{2}(C)^{-\infty}
$$

characterized by

$$
\langle v, \iota(u)\rangle:=\int_{C} v(x) \overline{u(x)} d \mu(x) \text { for any } v \in L^{\infty}(C) .
$$

The inclusion

$$
L^{2}(C)^{\infty} \subset L^{2}(C) \subset L^{2}(C)^{-\infty}
$$

is sometimes referred to as the Gelfand triple.

From the standard theory of Sobolev spaces and the definition of smooth vectors, we have the following inclusive relation:

$$
C_{0}^{\infty}(C) \subset L^{2}(C)^{\infty} \subset C^{\infty}(C) .
$$

(To see $L^{2}(C)^{\infty} \subset C^{\infty}(C)$, it is enough to use the $\bar{P}^{\max }$-action on $L^{2}(C)$.) Then, taking their duals, we get

$$
\mathcal{E}^{\prime}(C) \subset L^{2}(C)^{-\infty} \subset \mathcal{D}^{\prime}(C),
$$

where $\mathcal{D}^{\prime}(C)$ stands for the space of distributions on $C$, and $\mathcal{E}^{\prime}(C)$ for that of compactly supported distributions on $C$.

For $g \in G$, we extend the unitary operator $S=\pi(g)$ on $L^{2}(C)$ to a continuous operator $\widetilde{S}$ on $L^{2}(C)^{-\infty}$ by

$$
\langle v, \widetilde{S} u\rangle:=\left\langle S^{-1} v, u\right\rangle \quad \text { for } u \in L^{2}(C)^{-\infty} \text { and } v \in L^{2}(C)^{\infty} .
$$

Here, we have used the fact that the unitary operator $S^{-1}$ on $L^{2}(C)$ induces a continuous map (we use the same letter $S^{-1}$ ):

$$
S^{-1}: L^{2}(C)^{\infty} \rightarrow L^{2}(C)^{\infty} .
$$


Then, the extension $\widetilde{S}$ satisfies

$$
\widetilde{S} \circ \iota=\iota \circ S \text { on } L^{2}(C),
$$

because for $u \in L^{2}(C)$ and $v \in L^{\infty}(C)$ we have

$$
\begin{aligned}
\left\langle v, \widetilde{S}_{\iota}(u)\right\rangle & =\left\langle S^{-1} v, \iota(u)\right\rangle \\
& =\left(S^{-1} v, u\right)_{L^{2}(C)} \\
& =(v, S u)_{L^{2}(C)} \\
& =\langle v, \iota(S u)\rangle .
\end{aligned}
$$

Likewise, the Lie algebra $\mathfrak{g}$ acts on $L^{2}(C)^{-\infty}$ by

$$
\langle v, d \tilde{\pi}(X) u\rangle:=-\langle d \pi(X) v, u\rangle \quad \text { for } u \in L^{2}(C)^{-\infty} \text { and } v \in L^{2}(C)^{\infty} .
$$

Then, it readily follows that

$$
d \tilde{\pi}(\operatorname{Ad}(g) X)=\tilde{\pi}(g) d \tilde{\pi}(X) \tilde{\pi}\left(g^{-1}\right)
$$

for $g \in G$ and $X \in \mathfrak{g}$.

We shall write simply $S$ for $\widetilde{S}$, and $d \pi(X)$ for $d \tilde{\pi}(X)$ if there is no confusion.

For $\xi \in C$, we denote by $\delta_{\xi}$ the Dirac delta function at $\xi \in C$, namely,

$$
\left\langle v, \delta_{\xi}\right\rangle:=v(\xi)
$$

for a continuous function $v$ on $C$. Since

$$
\delta_{\xi}: L^{2}(C)^{\infty} \rightarrow \mathbb{C}
$$

is a continuous map, we may regard $\delta_{\xi} \in L^{2}(C)^{-\infty}$. Then, we have

$$
\mathcal{F}_{C}^{-1} \delta_{\xi} \in L^{2}(C)^{-\infty} \text {. }
$$

Applying the definition (2.5.10) to $S=\mathcal{F}_{C}^{-1}$, we have for any $v \in L^{\infty}(C)$,

$$
\begin{aligned}
\left\langle v, \mathcal{F}_{C}^{-1} \delta_{\xi}\right\rangle & =\left\langle\mathcal{F}_{C} v, \delta_{\xi}\right\rangle \\
& =\left(\mathcal{F}_{C} v\right)(\xi)
\end{aligned}
$$

Remark 2.5.1. In contrast to the (Euclidean) Fourier transform $\mathcal{F}_{\mathbb{R}^{n}}, \mathcal{F}_{C}^{-1} \delta_{\xi}$ is a real valued distribution. In other words, the kernel $k(x, \xi)=\frac{1}{(2 \pi)^{\frac{n}{2}}} e^{\sqrt{-1}\langle x, \xi\rangle}$ 
for $\mathcal{F}_{\mathbb{R}^{n}}$ is not real valued, while the kernel $K(x, \xi)$ for $\mathcal{F}_{C}$ below is real valued. This reflects the fact that both kernels $k(x, \xi)$ and $K(x, \xi)$ are characterized by the system of differential equations up to scalar (see Introduction 1.2):

$$
\begin{array}{lll}
p_{j} k(\cdot, \xi)=\xi_{j} k(\cdot, \xi) & \text { in } \mathbb{R}^{n} \quad & (1 \leq j \leq n), \\
P_{j} K(\cdot, \xi)=4 \xi_{j} K(\cdot, \xi) & \text { in } C & (1 \leq j \leq n),
\end{array}
$$

where $p_{j}=-\sqrt{-1} \frac{\partial}{\partial x_{j}}$. A distinguishing feature here is that the self-adjoint differential operator $p_{j}$ of first order satisfies

$$
\overline{p_{j}}=-p_{j},
$$

while the self-adjoint differential operator $P_{j}$ of second order satisfies

$$
\overline{P_{j}}=P_{j} .
$$

For a fixed $\xi \in C, \mathcal{F}_{C}^{-1} \delta_{\xi}$ is a distribution on $C$ by $(2.5 .13)$ and $(\overline{2.5 .9})$. We shall see that this distribution is locally integrable on the open dense subset

$$
\{x \in C:\langle x, \xi\rangle \neq 0\} .
$$

Thus, it is convenient to write the distribution $\mathcal{F}_{C}^{-1} \delta_{\xi}$ as a generalized function in the sense of Gelfand-Shilov 24] by using the canonical measure $d \mu(x)=\delta(Q(x))$ on $C$, that is,

$$
\left(\mathcal{F}_{C}^{-1} \delta_{\xi}\right)(x)=K(x, \xi) d \mu(x)
$$

for some generalized function $K(\cdot, \xi)$ on $C$. Then, the formula (2.5.14) can be expressed as

$$
\left(\mathcal{F}_{C} v\right)(\xi)=\int_{C} K(x, \xi) v(x) d \mu(x) .
$$

The argument so far assures that $K(\cdot, \xi)$ is a generalized function on $C$ for each fixed $\xi \in C$. On the other hand, since the sesqui-linear map

$$
L^{2}(C) \times L^{2}(C) \rightarrow \mathbb{C}, \quad(v, u) \mapsto\left(\mathcal{F}_{C} v, u\right)_{L^{2}(C)}
$$

is continuous, we may regard $K(x, \xi)$ is a generalized function (or equivalently, $K(x, \xi) d \mu(x) d \mu(\xi)$ is a distribution) on the direct product manifold $C \times C$ such that

$$
\left(\mathcal{F}_{C} v, u\right)_{L^{2}(C)}=\int_{C \times C} v(x) \overline{u(\xi)} K(x, \xi) d \mu(x) d \mu(\xi)
$$


for any $u, v \in L^{2}(C)^{\infty}$.

Now, we are ready to state basic properties of the 'unitary inversion operator' $\mathcal{F}_{C}=\pi\left(w_{0}\right)$ as the counterpart of the properties 10, $\mathbf{1 3}$ of the conformal inversion $w_{0} \in G$ :

\section{Theorem 2.5.2.}

1) $\mathcal{F}_{C}$ is an involutive unitary operator on $L^{2}(C)$. In particular, we have

$$
K(x, \xi)=\overline{K(\xi, x)}
$$

as distributions on $C \times C$.

2) If $v \in L^{2}(C)^{\infty}$, then $\mathcal{F}_{C} v \in L^{2}(C)^{\infty}$. In particular,

$$
\mathcal{F}_{C} v \in C^{\infty}(C) \cap L^{2}(C)
$$

for any compactly supported $C^{\infty}$ function $v$ on $C$.

3) For $1 \leq j \leq n$, the following identities of operators on $L^{2}(C)^{\infty}$ hold:

$$
\begin{aligned}
& \mathcal{F}_{C} \circ 4 x_{j}=P_{j} \circ \mathcal{F}_{C}, \\
& \mathcal{F}_{C} \circ P_{j}=4 x_{j} \circ \mathcal{F}_{C}, \\
& \mathcal{F}_{C} \circ E=-(E+n-2) \circ \mathcal{F}_{C} .
\end{aligned}
$$

These identities hold also on $L^{2}(C)^{-\infty}$.

4) For any $v \in L^{2}(C)^{\infty}$,

$$
\left(\mathcal{F}_{C} v\right)(\xi)=\int_{C} v(x) K(x, \xi) d \mu(x) .
$$

5) For each fixed $\xi=\left(\xi_{1}, \ldots, \xi_{n}\right) \in C$, the generalized function $K(\xi, \cdot)$ solves the system of differential equations on $C$ :

$$
P_{j} K(\xi, \cdot)=4 \xi_{j} K(\xi, \cdot) \quad(1 \leq j \leq n) .
$$

In turn, $K(\cdot, \xi)$ satisfies

$$
P_{j} K(\cdot, \xi)=4 \xi_{j} K(\cdot, \xi) \quad(1 \leq j \leq n) .
$$

Remark 2.5.3. We shall see in Theorem 5.1.1 that

$$
K(x, \xi)=K(\xi, x)
$$

as distributions on $C \times C$. 
Proof of Theorem 2.5.2. 1) The first statement is straightforward from definition $\mathcal{F}_{C}=\pi\left(w_{0}\right)$ because $\left(\pi, L^{2}(C)\right)$ is a unitary representation of $G$ (Fact 2.2.1) and $w_{0}^{2}=1$. The second statement follows from (2.5.16) and

$$
\left(\mathcal{F}_{C} v, u\right)=\left(\mathcal{F}_{C} v, \mathcal{F}_{C}^{2} u\right)=\left(v, \mathcal{F}_{C} u\right) .
$$

2) The group $G$ preserves $L^{2}(C)^{\infty}$, and in particular, we have

$$
\mathcal{F}_{C}\left(L^{2}(C)^{\infty}\right)=L^{2}(C)^{\infty} .
$$

Since $C_{0}^{\infty}(C) \subset L^{2}(C)^{\infty} \subset C^{\infty}(C) \cap L^{2}(C)$, we get $\mathcal{F}_{C}\left(C_{0}^{\infty}(C)\right) \subset C^{\infty}(C) \cap$ $L^{2}(C)$.

3) By (2.5.3) in 13, we have the following identities on $L^{2}(C)^{\infty}$, and also on $L^{2}(C)^{-\infty}$ by $(2.5 .12)$ :

$$
\begin{aligned}
& \mathcal{F}_{C} \circ d \pi\left(\bar{N}_{j}\right)=\epsilon_{j} d \pi\left(N_{j}\right) \circ \mathcal{F}_{C}, \\
& \mathcal{F}_{C} \circ d \pi\left(N_{j}\right)=\epsilon_{j} d \pi\left(\bar{N}_{j}\right) \circ \mathcal{F}_{C}, \\
& \mathcal{F}_{C} \circ d \pi(H)=-d \pi(H) \circ \mathcal{F}_{C} .
\end{aligned}
$$

Now we recall the formulas of the differential action:

$$
\begin{aligned}
& \left.d \pi\left(\bar{N}_{j}\right)=2 \sqrt{-1} x_{j} \quad \text { (see }(2.3 .15)\right), \\
& \left.d \pi\left(N_{j}\right)=\frac{\sqrt{-1}}{2} \epsilon_{j} P_{j} \quad \text { (see }(2.3 .19)\right), \\
& d \pi(H)=-\left(E+\frac{n-2}{2}\right) \quad(\text { see }(2.3 .14)) .
\end{aligned}
$$

These operators are defined on $L^{2}(C)^{\infty} \subset C^{\infty}(C)$, and in turn, they are defined on $L^{2}(C)^{-\infty} \subset \mathcal{D}^{\prime}(C)$ (see (2.5.9)). Therefore, the third statement follows.

4) Since $w_{0}^{-1}=w_{0}$, we have $\mathcal{F}_{C}^{-1}=\mathcal{F}_{C}$, and therefore the statement is equivalent to what we have seen in (2.5.15).

5) It follows from the third and fourth statements that we have (by switching $x$ and $\xi$ )

$$
\begin{aligned}
\int_{C} 4 \xi_{j} K(\xi, x) v(\xi) d \mu(\xi) & =\left(P_{j}\right)_{x} \int_{C} K(\xi, x) v(\xi) d \mu(\xi) \\
& =\int_{C}\left(P_{j}\right)_{x} K(\xi, x) v(\xi) d \mu(\xi)
\end{aligned}
$$

for any $v \in L^{2}(C)^{\infty}$. Hence, we have shown

$$
4 \xi_{j} K(\xi, x)=\left(P_{j}\right)_{x} K(\xi, x) .
$$

The second assertion follows from $\overline{P_{j}}=P_{j}$ as we saw in Remark 2.5.1. Thus, Theorem 2.5.2 has been proved. 
If $A$ is a continuous operator on $L^{2}(C)$, then $A$ induces a linear map (we use the same letter) $A: L^{2}(C)^{\infty} \rightarrow L^{2}(C)^{\infty}$ on the space of smooth vectors.

The next result shows that the intertwining relation in Theorem 2.5 .2 (3) characterizes the operator $\mathcal{F}_{C}$ up to scalar:

Theorem 2.5.4. Suppose $A$ is a continuous operator on $L^{2}(C)$ such that

$$
\begin{aligned}
& A \circ 4 x_{j}=P_{j} \circ A, \\
& A \circ P_{j}=4 x_{j} \circ A,
\end{aligned}
$$

on $L^{2}(C)^{\infty}$ for $1 \leq j \leq n$. Then, $A$ is a scalar multiple of $\mathcal{F}_{C}$. In particular, $A^{2}$ is a scalar multiple of the identity operator.

Proof. We set $B:=\mathcal{F}_{C}^{-1} \circ A$. Then, $B$ satisfies

$$
\begin{aligned}
& B \circ 4 x_{j}=4 x_{j} \circ B, \\
& B \circ P_{j}=P_{j} \circ B,
\end{aligned}
$$

on $L^{2}(C)^{\infty}$, and consequently,

$$
\begin{aligned}
& B \circ d \pi\left(\bar{N}_{j}\right)=d \pi\left(\bar{N}_{j}\right) \circ B, \\
& B \circ d \pi\left(N_{j}\right)=d \pi\left(N_{j}\right) \circ B,
\end{aligned}
$$

for $1 \leq j \leq n$. Since the vector space $\mathfrak{n}^{\max }+\overline{\mathfrak{n}^{\max }}=\sum_{j=1}^{n} \mathbb{R} N_{j}+\sum_{j=1}^{n} \mathbb{R} \bar{N}_{j}$ generates the whole Lie algebra $\mathfrak{g}$, we have

$$
B \circ d \pi(X)=d \pi(X) \circ B
$$

for any $X \in \mathfrak{g}$. In particular, such an operator $B$ preserves the space of $K$-finite vectors, namely,

$$
B\left(L^{2}(C)_{K}\right) \subset L^{2}(C)_{K} .
$$

Therefore, the identities (2.5.18) hold also on $L^{2}(C)_{K}$.

On the other hand, it follows from Fact 2.1.1 and Fact 2.2.1 that $G$ acts irreducibly on $L^{2}(C)$. Then, $L^{2}(C)_{K}$ is an irreducible $(\mathfrak{g}, K)$-module. Therefore, $B$ must be a scalar multiple of the identity by Schur's lemma. Hence, $A$ is a scalar multiple of $\mathcal{F}_{C}$. The last assertion is an immediate consequence of the fact that $\mathcal{F}_{C}^{2}=\mathrm{id}$ (see 10).

Theorem 2.5.5. For a fixed $\xi=\left(\xi_{1}, \ldots, \xi_{n}\right) \in \mathbb{R}^{n} \backslash\{0\}$, we consider the system of differential equations:

$$
P_{j} \psi=4 \xi_{j} \psi \quad(1 \leq j \leq n),
$$

as distributions on $C$. 
1) If $Q(\xi) \neq 0$, then $\psi=0$.

2) If $Q(\xi)=0$, then the solution space in $L^{2}(C)^{-\infty}$ is one-dimensional.

Proof. 1) It follows from (2.5.19) that

$$
\left(\sum_{j=1}^{n} \epsilon_{j} P_{j}^{2}\right) \psi=4 Q(\xi) \psi
$$

Then, by Theorem 2.4.1 (5), the left-hand side vanishes. Hence, $\psi=0$ unless $Q(\xi)=0$.

2) Suppose $\xi \in C$. Taking the Fourier transform $\mathcal{F}_{C}$ of the differential equation (2.5.19), we have the following equation

$$
4 x_{j}\left(\mathcal{F}_{C} \psi\right)=4 \xi_{j}\left(\mathcal{F}_{C} \psi\right) \text { in } L^{2}(C)^{-\infty}
$$

from Theorem 2.5 .2 (3). Hence,

$$
\left(x_{j}-\xi_{j}\right)\left(\mathcal{F}_{C} \psi\right)=0 \quad(1 \leq j \leq n) .
$$

Since $\mathcal{F}_{C} \psi \in L^{2}(C)^{-\infty} \subset \mathcal{D}^{\prime}(C)$, the equations (2.5.20) hold as distributions on $C$. Then, considering the equations $(2.5 .20)$ in a local chart, we see that $\mathcal{F}_{C} \psi$ is a scalar multiple of the delta function $\delta_{\xi}$. Conversely, $\delta_{\xi}$ solves $(2.5 .20)$ and $\delta_{\xi} \in L^{2}(C)^{-\infty}$. Therefore, $\mathcal{F}_{C}^{-1} \delta_{\xi} \in L^{2}(C)^{-\infty}$ solves (2.5.19). Now, Theorem is proved. 


\section{Chapter 3}

\section{$K$-finite eigenvectors in the Schrödinger model $L^{2}(C)$}

In the conformal model (see Section 2.1), we can find readily explicit $K$ finite vectors. However, it is far from being obvious to find explicit forms of $K$-finite vectors in the $L^{2}$-model for the minimal representation. In this chapter, generalizing the idea of [49, Theorem 5.8] for the minimal $K$-type, we find explicit vectors in $L^{2}(C)$ for every $K$-type, by carrying out the computation involving the integral operator $\mathcal{T}: L^{2}(C) \stackrel{\sim}{\rightarrow} \overline{V^{p, q}}$ (see Fact 2.2.1).

\subsection{Result of this chapter}

Throughout this chapter, we assume $p \geq q \geq 2$ and $p+q \geq 6$. For $(l, k) \in \mathbb{N}^{2}$, we consider the following two (non-exclusive) cases:

$$
\begin{aligned}
& \text { Case } 1: \frac{p-q}{2}+l-k \geq 0 \\
& \text { Case } 2: \frac{p-q}{2}+l-k \leq 0
\end{aligned}
$$

The case $\frac{p-q}{2}+l-k=0$ belongs to both Cases 1 and 2 . This overlap will be convenient later because all the formulas below are the same for both Cases 1 and 2 if $(l, k)$ satisfies $\frac{p-q}{2}+l-k=0$. 
For $(l, k) \in \mathbb{N}^{2}$, we define real analytic functions $f_{l, k}$ on $\mathbb{R}_{+}$by

$$
\begin{aligned}
f_{l, k}(r) & := \begin{cases}r^{-\frac{q-3}{2}+l} K_{\frac{q-3}{2}+k}(2 r) & \text { Case 1, } \\
r^{-\frac{p-3}{2}+k} K_{\frac{p-3}{2}+l}(2 r) & \text { Case 2, }\end{cases} \\
& =r^{l+k} \times \begin{cases}\widetilde{K}_{\frac{q-3}{2}+k}(2 r) & \text { Case 1, } \\
\widetilde{K}_{\frac{p-3}{2}+l}(2 r) & \text { Case 2. }\end{cases}
\end{aligned}
$$

Here, $K_{\nu}(z)$ is the $K$-Bessel function, i.e., the modified Bessel function of the second kind (see Appendix 7.2) and $\widetilde{K}_{\nu}(z)=\left(\frac{z}{2}\right)^{-\nu} K_{\nu}(z)$ is the normalized $K$-Bessel function (see (7.2.6)).

By using the polar coordinate (2.2.2), we define a linear subspace $H_{l, k}$ of $C^{\infty}(C)$ consisting of linear combinations of the following functions:

$$
f_{l, k}(r) \phi(\omega) \psi(\eta) \quad\left(\phi \in \mathcal{H}^{l}\left(\mathbb{R}^{p-1}\right), \psi \in \mathcal{H}^{k}\left(\mathbb{R}^{q-1}\right)\right) .
$$

Here, $\mathcal{H}^{j}\left(\mathbb{R}^{m}\right)$ denotes the space of spherical harmonics of degree $j$ (see Appendix (7.5).

We recall from Section 2.3 that there are two key compact subgroups for the analysis on the minimal representation $L^{2}(C)$ :

$$
\begin{array}{ll}
K & \simeq O(p) \times O(q), \\
K^{\prime}=K \cap M_{+}^{\max } & \simeq O(p-1) \times O(q-1) .
\end{array}
$$

We note that the $K^{\prime}$-action on $L^{2}(C)$ is just the pull-back of the $K^{\prime}$-action on $C$ (see $(2.3 .10)$ ), but the $K$-action on $L^{2}(C)$ is more complicated because $K$ cannot act on $C$. Then, here is our main result of this chapter:

Theorem 3.1.1. For each pair $(l, k) \in \mathbb{N}^{2}$, we have

1) (asymptotic behavior) $H_{l, k} \subset L^{2}(C)$ for any $l, k \in \mathbb{N}$.

2) (K-type and $K^{\prime}$-type) $H_{l, k} \simeq \mathcal{H}^{l}\left(\mathbb{R}^{p-1}\right) \otimes \mathcal{H}^{k}\left(\mathbb{R}^{q-1}\right)$ as a $K^{\prime}$-module. Furthermore, $H_{l, k}$ belongs to the $K$-type $\mathcal{H}^{a(l, k)}\left(\mathbb{R}^{p}\right) \otimes \mathcal{H}^{a(l, k)+\frac{p-q}{2}}\left(\mathbb{R}^{q}\right)$ of $L^{2}(C)$. Here, we define a non-negative integer $a(l, k)$ by

$$
a(l, k):=\max \left(l, k-\frac{p-q}{2}\right)= \begin{cases}l & \text { Case 1, } \\ k-\frac{p-q}{2} & \text { Case 2. }\end{cases}
$$

3) (eigenspace of $\left.\pi\left(w_{0}\right)\right) \pi\left(w_{0}\right)$ acts on $H_{l, k}$ by the scalar $(-1)^{a(l, k)+\frac{p-q}{2}}$.

4) (intertwining operator) Fix any $\phi \in \mathcal{H}^{l}\left(\mathbb{R}^{p-1}\right), \quad \psi \in \mathcal{H}^{k}\left(\mathbb{R}^{q-1}\right)$, and set

$$
u_{l, k}(r \omega, r \eta):=f_{l, k}(r) \phi(\omega) \psi(\eta) \in H_{l, k} .
$$


Then $\mathcal{T}: L^{2}(C) \rightarrow \overline{V^{p, q}}$ has the following form on the subspace $H_{l, k}$ :

$$
\mathcal{T} u_{l, k}=c_{l, k} I_{l \rightarrow a(l, k)}^{p}(\phi) I_{k \rightarrow a(l, k)+\frac{p-q}{2}}^{q}(\psi),
$$

where $I_{i \rightarrow j}^{m}: \mathcal{H}^{i}\left(\mathbb{R}^{m-1}\right) \rightarrow \mathcal{H}^{j}\left(\mathbb{R}^{m}\right)(0 \leq i \leq j)$ is an $O(m-1)$-homomorphism defined in Fact 7.5.1, and the constant $c_{l, k}$ is given by

$$
c_{l, k}:=\frac{\sqrt{-1}^{l+k}}{\sqrt{\pi}} \times \begin{cases}\frac{\Gamma\left(\frac{p-q}{2}+l-k+1\right)}{2^{\frac{p-q}{2}+l-k+3} \Gamma\left(\frac{p-2}{2}+l\right)} & \text { Case 1, } \\ \frac{\Gamma\left(-\frac{p-q}{2}-l+k+1\right)}{2^{-\left(\frac{p-q}{2}+l-k\right)+3} \Gamma\left(\frac{q-2}{2}+k\right)} & \text { Case 2. }\end{cases}
$$

5) (L'-norm) For any $(l, k) \in \mathbb{N}^{2}$,

$$
\left\|\mathcal{T} u_{l, k}\right\|_{L^{2}(M)}^{2}=\frac{1}{2 a(l, k)+p-2}\left\|u_{l, k}\right\|_{L^{2}(C)}^{2} .
$$

A special case (the $k=0$ case) of Theorem 3.1.1 (2) provides us an explicit $K$-finite vector for every $K$-type.

Corollary 3.1.2. For each $a \in \mathbb{N}$, the function

$$
r^{a-\frac{q-2}{2}} K_{\frac{q-2}{2}}(2 r) \phi(\omega)
$$

is a $K$-finite vector for any $\phi \in \mathcal{H}^{a}\left(\mathbb{R}^{p-1}\right)$. More precisely, it belongs to the K-type

$$
\mathcal{H}^{a}\left(\mathbb{R}^{p}\right) \otimes \mathcal{H}^{a+\frac{p-q}{2}}\left(\mathbb{R}^{q}\right) .
$$

Proof. Under our assumption $p \geq q, a(l, 0)=l$ by the definition (3.1.5) because $(l, 0)$ belongs to Case 1 . Hence, Corollary follows immediately from Theorem 3.1.1 (2).

By using the unitary inner product $(,)_{M}($ see $(2.1 .7))$ for the conformal model $\left(\varpi^{p, q}, \overline{V^{p, q}}\right)$ of the minimal representation $G=O(p, q)$, the formula (3.1.9) in Theorem 3.1.1 (5) can be restated as

$$
\left(\mathcal{T} u_{l, k}, \mathcal{T} u_{l, k}\right)_{M}=\frac{1}{2}\left\|u_{l, k}\right\|_{L^{2}(C)}^{2} .
$$

Remark 3.1.3. Theorem 3.1.1 (2) for $l=k=0$ (or Corollary 3.1.2 for $a=0)$ was proved in [49, Theorem 5.8]. Since $p \geq q$, we are dealing with Case 1 if $l=k=0$ and $a(0,0)=0$. In this particular case, Theorem 3.1.1 (2) asserts that $f_{0,0}(r)=r^{-\frac{q-3}{2}} K_{\frac{q-3}{2}}(2 r)$ belongs to the minimal $K$-type $\mathcal{H}^{0}\left(\mathbb{R}^{p}\right) \otimes \mathcal{H}^{\frac{p-q}{2}}\left(\mathbb{R}^{q}\right)$ of $\left(\pi, L^{2}(C)\right)$. 
Remark 3.1.4. For $q=2, \pi$ is essentially a highest weight module (see Section 1.7 for the $n_{2}=1$ case). We note that for $q=2, \mathcal{H}^{k}\left(\mathbb{R}^{q-1}\right)$ is nonzero only if $k=0$ or 1 (see Appendix 7.5). Thus our assumption $p+q \geq 6$ combined with $q=2$ and $k=0,1$ implies $\frac{p-q}{2}+l-k=\frac{p+q}{2}-2+l-k \geq 0$. Hence, the pairs $(l, k)(k=0,1)$ belong automatically to Case 1. It then follows from the definition (3.1.2) that

$$
\begin{aligned}
& f_{l, 0}(r)=r^{\frac{1}{2}+l} K_{-\frac{1}{2}}(2 r), \\
& f_{l, 1}(r)=r^{\frac{1}{2}+l} K_{\frac{1}{2}}(2 r) .
\end{aligned}
$$

By using the formula

$$
K_{\frac{1}{2}}(z)=K_{-\frac{1}{2}}(z)=\left(\frac{\pi}{2 z}\right)^{\frac{1}{2}} e^{-z}
$$

(see (7.2.2)), we get

$$
f_{l, 0}(r)=f_{l, 1}(r)=\frac{\sqrt{\pi}}{2} r^{l} e^{-2 r} .
$$

We note that $f_{l, 0}(r)\left(=f_{l, 1}(r)\right)$ coincides with the function ' $f_{l, l}(r)$ ' in 46 , (3.2.4)] up to a constant multiple. (In [46], we investigated the Schrödinger model for $O(p, 2)$, and the function ' $f_{l, l}(r)$ ' was defined by using the Laguerre polynomial. The definition of ' $f_{l, l}(r)$ ' is different from (3.1.2).)

Our method to show Theorem 3.1.1 is based on the technique of [49, $\S 5.6, \S 5.7]$. The key lemma is Lemma 7.8.1, which gives a formula of the Hankel transform of the $K$-Bessel function with trigonometric parameters by means of the Gegenbauer polynomial.

The subspace $\bigoplus_{l, k \in \mathbb{N}} H_{l, k}$ is not dense in $L^{2}(C)$, but is large enough (see Section 3.2) that we can make use of Theorem 3.1.1 for the proof of Theorem 4.1 .1 in Chapter 4 (see also Section 4.1 for its idea).

\section{2 $K \cap M^{\max }$-invariant subspaces $H_{l, k}$}

The subspace $\bigoplus_{l, k \in \mathbb{N}} H_{l, k}$ is not dense in $L^{2}(C)$, but serves as a 'skeleton'. In this section, we try to clarify its meaning. 
We begin with the branching law $G \downarrow K$ (see (2.1.6)) and $K \downarrow K^{\prime}\left(K^{\prime}\right.$ denotes $\left.K^{\prime}:=K \cap M_{+}^{\max } \simeq O(p-1) \times O(q-1)\right)$ :

$$
\begin{aligned}
L^{2}(C)_{K} & \simeq \bigoplus_{a=0}^{\infty} \mathcal{H}^{a}\left(\mathbb{R}^{p}\right) \otimes \mathcal{H}^{a+\frac{p-q}{2}}\left(\mathbb{R}^{q}\right) \\
& \simeq \bigoplus_{a=0}^{\infty} \bigoplus_{l=0}^{a} \bigoplus_{k=0}^{a+\frac{p-q}{2}} \mathcal{H}^{l}\left(\mathbb{R}^{p-1}\right) \otimes \mathcal{H}^{k}\left(\mathbb{R}^{q-1}\right)
\end{aligned}
$$

The irreducible decomposition (3.2.1) shows that $L^{2}(C)_{K}$ is multiplicity-free as a $K$-module. Hereafter, we identify the $K$-module $\mathcal{H}^{a}\left(\mathbb{R}^{p}\right) \otimes \mathcal{H}^{a+\frac{p-q}{2}}\left(\mathbb{R}^{q}\right)$ with the corresponding subspace of $L^{2}(C)_{K}$. Then we observe:

S1 ( $K^{\prime}$-type) Fix a pair $(l, k) \in \mathbb{N}^{2}$. In light of (3.2.1) and (3.2.2), the $K^{\prime}$ type $\mathcal{H}^{l}\left(\mathbb{R}^{p-1}\right) \otimes \mathcal{H}^{k}\left(\mathbb{R}^{q-1}\right)$ occurs in the $K$-module $\mathcal{H}^{a}\left(\mathbb{R}^{p}\right) \otimes \mathcal{H}^{a+\frac{p-q}{2}}\left(\mathbb{R}^{q}\right)$ if and only if $a \geq a(l, k)$. Further, $H_{l, k}$ is characterized as a subspace of $L^{2}(C)$ satisfying the following two conditions:

$$
\left\{\begin{array}{l}
W \simeq \mathcal{H}^{l}\left(\mathbb{R}^{p-1}\right) \otimes \mathcal{H}^{k}\left(\mathbb{R}^{q-1}\right) \text { as } K^{\prime} \text {-modules, } \\
W \subset \mathcal{H}^{a(l, k)}\left(\mathbb{R}^{p}\right) \otimes \mathcal{H}^{a(l, k)+\frac{p-q}{2}}\left(\mathbb{R}^{q}\right) .
\end{array}\right.
$$

S2 (K-type) Fix $a \in \mathbb{N}$. Then for $(l, k) \in \mathbb{N}^{2}$,

$$
\left(\bigoplus_{l, k \in \mathbb{N}} H_{l, k}\right) \cap\left(\mathcal{H}^{a}\left(\mathbb{R}^{p}\right) \otimes \mathcal{H}^{a+\frac{p-q}{2}}\left(\mathbb{R}^{q}\right)\right)
$$

is non-zero if and only if at least one of $l$ and $k$ attains its maximum in the set $\left\{\left(l^{\prime}, k^{\prime}\right) \in \mathbb{N}^{2}: 0 \leq l^{\prime} \leq a, 0 \leq k^{\prime} \leq a+\frac{p-q}{2}\right\}$ or equivalently, in the set of the $K^{\prime}$-types $\left(l^{\prime}, k^{\prime}\right)$ occurring in $\mathcal{H}^{a}\left(\mathbb{R}^{p}\right) \otimes \mathcal{H}^{a+\frac{p-q}{2}}\left(\mathbb{R}^{q}\right)$ (see the black dots - in the figure below). 


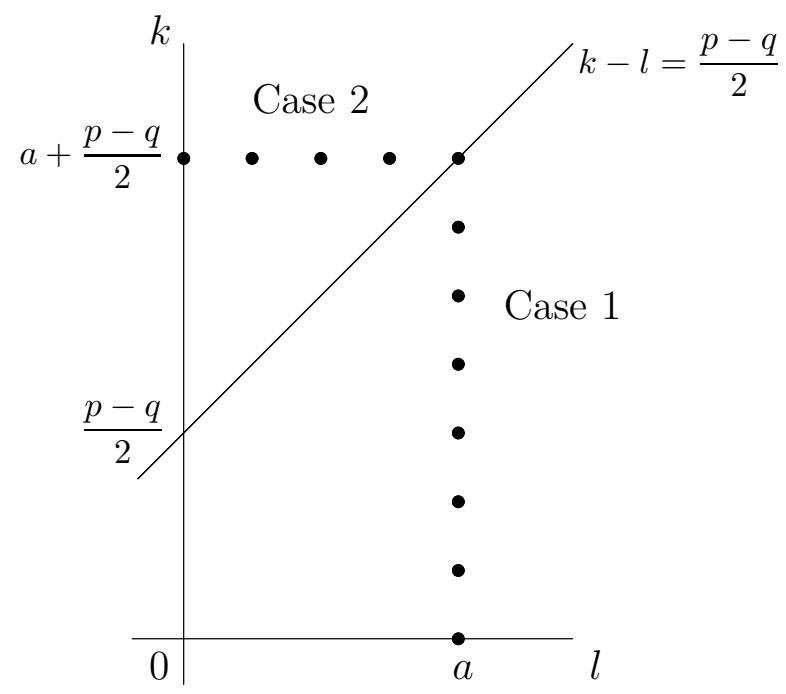

Figure 3.2.1

\subsection{Integral formula for the $K \cap M^{\max }$-intertwiner}

As a preparation for Theorem 3.1.1, we rewrite the integral formula for the $G$-isomorphism $\mathcal{T}: L^{2}(C) \rightarrow \overline{V^{p, q}}\left(\subset L^{2}(M)\right.$ ) (see Fact 2.2.1) applied to spherical harmonics. The main result of this section is Lemma 3.3.1.

We write $v \in M=S^{p-1} \times S^{q-1} \subset \mathbb{R}^{p+q}$ as

$$
v={ }^{t}\left(v_{0}, v^{\prime}, v^{\prime \prime}, v_{p+q-1}\right), \quad v_{0}, v_{p+q-1} \in \mathbb{R}, v^{\prime} \in \mathbb{R}^{p-1}, v^{\prime \prime} \in \mathbb{R}^{q-1}
$$

satisfying

$$
v_{0}^{2}+\left|v^{\prime}\right|^{2}=\left|v^{\prime \prime}\right|^{2}+v_{p+q-1}^{2}=1 .
$$

Lemma 3.3.1. Suppose that $u \in L^{2}(C)$ is of the form

$$
u(r \omega, r \eta)=f(r) \phi(\omega) \psi(\eta),
$$

for $\phi \in \mathcal{H}^{l}\left(\mathbb{R}^{p-1}\right), \psi \in \mathcal{H}^{k}\left(\mathbb{R}^{q-1}\right)$, and $f \in L^{2}\left(\mathbb{R}_{+}, r^{p+q-5} d r\right)$ with regard to the polar coordinate (2.2.2). Then, $\mathcal{T} u$ is reduced to the following integral transform of one variable: for $v \in M$ such that $v_{0}+v_{p+q-1}>0$,

$$
\begin{aligned}
& (\mathcal{T} u)(v)=e^{-\frac{\sqrt{-1}(l+k) \pi}{2}} \frac{\left|v^{\prime}\right|^{-\frac{p-3}{2}}\left|v^{\prime \prime}\right|^{-\frac{q-3}{2}}}{v_{0}+v_{p+q-1}} \phi\left(\frac{v^{\prime}}{\left|v^{\prime}\right|}\right) \psi\left(\frac{v^{\prime \prime}}{\left|v^{\prime \prime}\right|}\right) \\
& \times \int_{0}^{\infty} f(r) J_{\frac{p-3}{2}+l}\left(\frac{2\left|v^{\prime}\right| r}{v_{0}+v_{p+q-1}}\right) J_{\frac{q-3}{2}+k}\left(\frac{2\left|v^{\prime \prime}\right| r}{v_{0}+v_{p+q-1}}\right) r^{\frac{p+q-4}{2}} d r,
\end{aligned}
$$


where $J_{\nu}(z)$ denotes the Bessel function.

Proof. We recall from (2.2.7) that $\mathcal{T}=\left(\widetilde{\Psi}^{*}\right)^{-1} \circ \mathcal{F}^{-1} \circ T$. Let us compute $\left(\mathcal{F}^{-1} \circ T u\right)(z)$ for $z \in \mathbb{R}^{p+q-2}$. By the formula (2.2.3) and (2.2.6) for $T u=$ $u \delta(Q)$, we have

$$
\begin{aligned}
& 2\left(\mathcal{F}^{-1} \circ T u\right)(z) \\
= & (2 \pi)^{-\frac{p+q-2}{2}} \int_{0}^{\infty} \int_{S^{p-2}} \int_{S^{q-2}} f(r) \phi(\omega) \psi(\eta) e^{-\sqrt{-1}\left(\left\langle z^{\prime}, r \omega\right\rangle+\left\langle z^{\prime \prime}, r \eta\right\rangle\right)} r^{p+q-5} d r d \omega d \eta \\
= & \sqrt{-1}^{-(l+k)}\left|z^{\prime}\right|^{-\frac{p-3}{2}}\left|z^{\prime \prime}\right|^{-\frac{q-3}{2}} \phi\left(\frac{z^{\prime}}{\left|z^{\prime}\right|}\right) \psi\left(\frac{z^{\prime \prime}}{\left|z^{\prime \prime}\right|}\right) \\
& \times \int_{0}^{\infty} f(r) J_{\frac{p-3}{2}+l}\left(r\left|z^{\prime}\right|\right) J_{\frac{q-3}{2}+k}\left(r\left|z^{\prime \prime}\right|\right) r^{\frac{p+q-4}{2}} d r .
\end{aligned}
$$

Here, in (3.3.3), we used the following formula (see e.g. [31, Introduction, Lemma 3.6]):

$$
\int_{S^{m-1}} e^{\sqrt{-1} \lambda\langle\eta, \omega\rangle} \phi(\omega) d \omega=(2 \pi)^{\frac{m}{2}} \sqrt{-1} \phi(\eta) \frac{J_{l-1+\frac{m}{2}}(\lambda)}{\lambda^{\frac{m}{2}}-1} .
$$

Then, by the definition $(2.1 .9)$ of the pull-back $\left(\tilde{\Psi}^{*}\right)^{-1}$, we get the desired result (3.3.2).

\section{4 $K$-finite vectors $f_{l, k}$ in $L^{2}(C)$}

In this section, we collect basic results on the real analytic functions $f_{l, k}$ defined in (3.1.2).

Lemma 3.4.1. $f_{l, k} \in L^{2}\left(\mathbb{R}_{+}, \frac{1}{2} r^{p+q-5} d r\right)$.

Proof. The $K$-Bessel function decays exponentially at infinity. The asymptotic formula (see Fact 7.2.1 (2) in Appendix) implies

$$
f_{l, k}(r) \sim \begin{cases}c r^{-\frac{q-2}{2}+l} e^{-2 r} & \text { Case 1, } \\ c r^{-\frac{p-2}{2}+k} e^{-2 r} & \text { Case 2, }\end{cases}
$$

as $r \rightarrow \infty$. On the other hand, since $\widetilde{K}_{\nu}(r)=O\left(r^{-2 \nu}\right)$ as $r$ tends to 0 (see $(7.2 .10))$,

$$
f_{l, k}= \begin{cases}O\left(r^{l-k-q+3}\right) & \text { Case 1, } \\ O\left(r^{-l+k-p+3}\right) & \text { Case 2, }\end{cases}
$$

as $r$ tends to 0 . In either case, $f_{l, k}=O\left(r^{\frac{-p-q}{2}+3}\right)$ by the definition (3.1.1) of Cases 1 and 2 . Hence, we have $f_{l, k} \in L^{2}\left(\mathbb{R}_{+}, \frac{1}{2} r^{p+q-5} d r\right)$ for $p+q>4$. 
We shall give a finer estimate on the derivatives of $f_{l, k}$ in Lemma 4.4.1. The explicit formula of the $L^{2}$-norm of $f_{l, k}$ is obtained by the integration formula (7.2.13) of $K$-Bessel functions as follows:

\section{Proposition 3.4.2.}

$$
\begin{aligned}
& \left\|f_{l, k}\right\|_{L^{2}\left(\mathbb{R}_{+}, \frac{1}{2} r^{p+q-5} d r\right)}^{2} \\
& = \begin{cases}\frac{\Gamma\left(\frac{p-1}{2}+l\right)^{2} \Gamma\left(\frac{p+q-4}{2}+l+k\right) \Gamma\left(\frac{p-q+2}{2}+l-k\right)}{16 \Gamma(p-1+2 l)} & \text { Case 1, } \\
\frac{\Gamma\left(\frac{q-1}{2}+k\right)^{2} \Gamma\left(\frac{p+q-4}{2}+k+l\right) \Gamma\left(\frac{q-p+2}{2}+k-l\right)}{16 \Gamma(q-1+2 k)} & \text { Case 2. }\end{cases}
\end{aligned}
$$

Here is another integral formula that we use later:

\section{Lemma 3.4.3.}

$$
\begin{aligned}
& \int_{0}^{\infty} r^{\frac{p+q-6}{2}+\sqrt{-1} x} f_{l, k}(r) d r \\
& = \begin{cases}\frac{1}{4} \Gamma\left(\frac{p+q-4}{4}+\frac{l+k+\sqrt{-1} x}{2}\right) \Gamma\left(\frac{p-q}{4}+\frac{l-k+1+\sqrt{-1} x}{2}\right) & \text { Case 1, } \\
\frac{1}{4} \Gamma\left(\frac{p+q-4}{4}+\frac{k+l+\sqrt{-1} x}{2}\right) \Gamma\left(\frac{q-p}{4}+\frac{k-l+1+\sqrt{-1} x}{2}\right) & \text { Case 2. }\end{cases}
\end{aligned}
$$

Proof of Lemma 3.4.3. Apply the formula (7.2.11) of the Mellin transform of $K$-Bessel functions.

In order to compute $\mathcal{T} u_{l, k}$ explicitly by using the integral formula (3.3.2), we need yet another lemma:

Lemma 3.4.4. For a pair $(l, k) \in \mathbb{N}^{2}$, let $f_{l, k}$ be the function on $C$ defined in (3.1.2). With respect to the coordinate ${ }^{t}\left(v_{0}, v^{\prime}, v^{\prime \prime}, v_{p+q-1}\right) \in M \subset \mathbb{R}^{p+q}$ (see (3.3.1) ), the integral

$$
\int_{0}^{\infty} f_{l, k}(r) J_{\frac{p-3}{2}+l}\left(\frac{2\left|v^{\prime}\right| r}{v_{0}+v_{p+q-1}}\right) J_{\frac{q-3}{2}+k}\left(\frac{2\left|v^{\prime \prime}\right| r}{v_{0}+v_{p+q-1}}\right) r^{\frac{p+q-4}{2}} d r
$$

is equal to:

$$
\begin{array}{ll}
\frac{\Gamma\left(\frac{p-q}{2}+l-k+1\right)}{2^{\frac{p-q}{2}+l-k+3} \sqrt{\pi}}\left(v_{0}+v_{p+q-1}\right)\left|v^{\prime}\right|^{\frac{p-3}{2}+l}\left|v^{\prime \prime}\right|^{\frac{q-3}{2}+k} \widetilde{C}_{\frac{p-q}{2}+l-k}^{\frac{q-2}{2}+k}\left(v_{p+q-1}\right) & \text { Case 1, } \\
\frac{\Gamma\left(-\frac{p-q}{2}-l+k+1\right)}{2^{-\frac{p-q}{2}-l+k+3} \sqrt{\pi}}\left(v_{0}+v_{p+q-1}\right)\left|v^{\prime}\right|^{\frac{p-3}{2}+l}\left|v^{\prime \prime}\right|^{\frac{q-3}{2}+k} \widetilde{C}_{-\left(\frac{p-2}{2}+l\right.}^{\left.\frac{p-q}{2}+l-k\right)}\left(v_{0}\right) & \text { Case 2. }
\end{array}
$$


Proof of Lemma 3.4.4. We treat Case 1 first. By the change of variables $t:=2 r$, the integral (3.4.4) amounts to

$$
\frac{1}{2^{\frac{p+1}{2}+l}} \int_{0}^{\infty} t^{\frac{p-1}{2}+l} J_{\frac{p-3}{2}+l}\left(\frac{\left|v^{\prime}\right| t}{v_{0}+v_{p+q-1}}\right) J_{\frac{q-3}{2}+k}\left(\frac{\left|v^{\prime \prime}\right| t}{v_{0}+v_{p+q-1}}\right) K_{\frac{q-3}{2}+k}(t) d t .
$$

Applying Lemma 7.8.1 with

$$
\mu:=\frac{p-3}{2}+l, \quad \nu:=\frac{q-3}{2}+k, \quad \cos \theta:=v_{0}, \quad \cos \phi:=v_{p+q-1},
$$

we get the formula in Case 1 .

The proof for Case 2 goes similarly. In this case, the integral amounts to

$$
\frac{1}{2^{\frac{q+1}{2}+k}} \int_{0}^{\infty} t^{\frac{q-1}{2}+k} J_{\frac{p-3}{2}+l}\left(\frac{\left|v^{\prime}\right| t}{v_{0}+v_{p+q-1}}\right) J_{\frac{q-3}{2}+k}\left(\frac{\left|v^{\prime \prime}\right| t}{v_{0}+v_{p+q-1}}\right) K_{\frac{p-3}{2}+l}(t) d t
$$

by the change of variables $t:=2 r$. Now, we substitute $\mu:=\frac{q-3}{2}+k$, $\nu:=\frac{p-3}{2}+l, \cos \theta:=v_{p+q-1}$, and $\cos \phi:=v_{0}$ into (7.8.1).

\subsection{Proof of Theorem 3.1.1}

In this section, we complete the proof of Theorem 3.1.1.

Proof of Theorem 3.1.1. 1) By the isomorphism

$$
L^{2}(C) \simeq L^{2}\left(\mathbb{R}_{+}, \frac{1}{2} r^{p+q-5} d r\right) \widehat{\otimes} L^{2}\left(S^{p-2}\right) \widehat{\otimes} L^{2}\left(S^{q-2}\right) \quad(\text { see }(2.2 .5))
$$

in the polar coordinate, the first statement follows immediately from Lemma 3.4.1.

4) By (3.3.2) and Lemma 3.4.4, we have

$\mathcal{T} u_{l, k}(v)= \begin{cases}c_{l, k} \Gamma\left(\frac{p-2}{2}+l\right)\left|v^{\prime}\right|^{l} \phi\left(\frac{v^{\prime}}{\left|v^{\prime}\right|}\right)\left|v^{\prime \prime}\right|^{k} \psi\left(\frac{v^{\prime \prime}}{\left|v^{\prime \prime}\right|}\right) \widetilde{C}_{\frac{p-q}{2}+l-k}^{\frac{q-2}{2}+k}\left(v_{p+q-1}\right) & \text { Case 1, } \\ c_{l, k} \Gamma\left(\frac{q-2}{2}+k\right)\left|v^{\prime}\right|^{l} \phi\left(\frac{v^{\prime}}{\left|v^{\prime}\right|}\right) \widetilde{C}_{-\left(\frac{p-q}{2}+l-k\right)}^{\frac{p-2}{2}+l}\left(v_{0}\right)\left|v^{\prime \prime}\right|^{k} \psi\left(\frac{v^{\prime \prime}}{\left|v^{\prime \prime}\right|}\right) & \text { Case 2, }\end{cases}$

where $c_{l, k}$ is the constant defined in (3.1.8) and $v={ }^{t}\left(v_{0}, v^{\prime}, v^{\prime \prime}, v_{p+q-1}\right) \in$ $S^{p-1} \times S^{q-1}$. Now we use the definition (7.5.1) that

$$
I_{i \rightarrow j}^{m}(\phi)\left(x_{0}, x^{\prime}\right)=\left|x^{\prime}\right|^{i} \phi\left(\frac{x^{\prime}}{\left|x^{\prime}\right|}\right) \widetilde{C}_{j-i}^{\frac{m-2}{2}+i}\left(x_{0}\right)
$$


for $\left(x_{0}, x^{\prime}\right) \in S^{m-1}$, and in particular, for $i=j$,

$$
I_{j \rightarrow j}^{m}(\phi)\left(x_{0}, x^{\prime}\right)=\Gamma\left(\frac{m-2}{2}+j\right)\left|x^{\prime}\right|^{j} \phi\left(\frac{x^{\prime}}{\left|x^{\prime}\right|}\right)
$$

by (7.5.5). Thus, the formula (3.1.7) follows.

2) The first statement is obvious. Since $I_{i \rightarrow j}^{m}$ maps $\mathcal{H}^{i}\left(\mathbb{R}^{m-1}\right)$ into $\mathcal{H}^{j}\left(\mathbb{R}^{m}\right)$ (see Fact 7.5.1), (3.1.7) implies

$$
\mathcal{T} H_{l, k} \subset \mathcal{H}^{a(l, k)}\left(\mathbb{R}^{p}\right) \otimes \mathcal{H}^{a(l, k)+\frac{p-q}{2}}\left(\mathbb{R}^{q}\right) .
$$

Hence, we have proved the second statement.

3) By $(2.1 .5)$, the unitary inversion operator $\varpi^{p, q}\left(w_{0}\right)$ on $\overline{V^{p, q}}$ is given by

$$
\left(\varpi^{p, q}\left(w_{0}\right) h\right)\left(v^{\prime}, v^{\prime \prime}\right)=h\left(v^{\prime},-v^{\prime \prime}\right) .
$$

On the other hand, it is easy to see $h(-x)=(-1)^{j} h(x)$ for $h \in \mathcal{H}^{j}\left(\mathbb{R}^{q}\right)$ (see Appendix 7.5, H1). Therefore, $\varpi^{p, q}\left(w_{0}\right)$ acts on each $K$-type component $\mathcal{H}^{a}\left(\mathbb{R}^{p}\right) \otimes \mathcal{H}^{a+\frac{p-q}{2}}\left(\mathbb{R}^{q}\right)$ by a scalar multiple $(-1)^{a+\frac{p-q}{2}}$. Since $\mathcal{T}: L^{2}(C) \rightarrow$ $\overline{V^{p, q}}$ intertwines $\pi$ and $\varpi^{p, q}, \pi\left(w_{0}\right)$ acts on $H_{l, k}$ as the scalar $(-1)^{a+\frac{p-q}{2}}$ because $H_{l, k}$ belongs to the $K$-type $\mathcal{H}^{a(l, k)}\left(\mathbb{R}^{p}\right) \otimes \mathcal{H}^{a(l, k)+\frac{p-q}{2}}\left(\mathbb{R}^{q}\right)$. Thus, the third statement is proved.

5) We claim that the following formula holds:

$$
\left\|\mathcal{T} u_{l, k}\right\|_{L^{2}\left(S^{p-1} \times S^{q-1}\right)}^{2}=b_{l, k} \Gamma\left(k+l+\frac{p+q}{2}-2\right)\|\phi\|_{L^{2}\left(S^{p-1}\right)}^{2}\|\psi\|_{L^{2}\left(S^{q-1}\right)}^{2},
$$

where

$$
b_{l, k}= \begin{cases}\frac{\pi \Gamma(p-2+2 l) \Gamma\left(\frac{p-q}{2}+l-k+1\right)}{2^{2 p+4 l} \Gamma\left(l+\frac{p}{2}\right)^{2}} & \text { Case 1, } \\ \frac{\pi \Gamma(q-2+2 k) \Gamma\left(k-l-\frac{p-q}{2}+l\right)}{2^{2 q+4 k} \Gamma\left(k+\frac{q}{2}\right)^{2}} & \text { Case 2. }\end{cases}
$$

To see this, we begin with (3.1.7):

$\left\|\mathcal{T} u_{l, k}\right\|_{L^{2}\left(S^{p-1} \times S^{q-1}\right)}^{2}=\left|c_{l, k}\right|^{2}\left\|I_{l \rightarrow a(l, k)}^{p}(\phi)\right\|_{L^{2}\left(S^{p-1}\right)}^{2}\left\|I_{k \rightarrow a(l, k)+\frac{p-q}{2}}^{q}(\psi)\right\|_{L^{2}\left(S^{q-1}\right)}^{2}$.

By (3.1.7) and the norm formula (7.5.2), the right-hand side amounts to

$$
\begin{aligned}
& \left|c_{l, k}\right|^{2} \frac{2^{3-p-2 l} \pi \Gamma(p-2+l+a(l, k))}{(a(l, k)-l) !\left(a(l, k)+\frac{p-2}{2}\right)}\|\phi\|_{L^{2}\left(S^{p-1}\right)}^{2} \\
& \times \frac{2^{3-q-2 k} \pi \Gamma\left(q-2+k+a(l, k)+\frac{p-q}{2}\right)}{\left(a(l, k)+\frac{p-q}{2}-k\right) !\left(a(l, k)+\frac{p-q}{2}+\frac{q-2}{2}\right)}\|\psi\|_{L^{2}\left(S^{q-1}\right)}^{2} .
\end{aligned}
$$


Now, substituting (3.1.5) and (3.1.8) into this formula, we get (3.5.1) by elementary computations.

Finally, comparing (3.5.1) with Proposition 3.4.2, we obtain (3.1.9) by the Gauss duplication formula (7.4.3) of gamma functions. Hence, Theorem 3.1.1 is proved. 


\section{Chapter 4}

\section{Radial part of the inversion}

The goal of this chapter is to find the 'radial part' $T_{l, k}$ of the unitary inversion operator $\mathcal{F}_{C}: L^{2}(C) \rightarrow L^{2}(C)$. The main result of this chapter is Theorem 4.1.1.

\subsection{Result of this chapter}

Suppose $p \geq q \geq 2$ and $p+q \geq 6$. We recall from Section 2.2 that the polar coordinate of the (generalized) light cone $C$ :

$$
\mathbb{R}_{+} \times S^{p-2} \times S^{q-2} \simeq C, \quad(r, \omega, \eta) \mapsto(r \omega, r \eta)
$$

induces the isomorphism of Hilbert spaces:

$$
L^{2}(C) \simeq L^{2}\left(\mathbb{R}_{+}, \frac{1}{2} r^{p+q-5} d r\right) \widehat{\otimes} L^{2}\left(S^{p-2}\right) \widehat{\otimes} L^{2}\left(S^{q-2}\right) .
$$

This isomorphism respects the action of the compact group

$$
K^{\prime}:=K \cap M_{+}^{\max } \simeq O(p-1) \times O(q-1) .
$$

Since the Hilbert space $L^{2}\left(S^{p-2}\right)$ is decomposed into the direct Hilbert sum of spherical harmonics (see $\mathbf{H} \underline{\mathbf{6}}$ in Appendix 7.5 ):

$$
L^{2}\left(S^{p-2}\right) \simeq \sum_{j=0}^{\infty} \mathcal{H}^{j}\left(\mathbb{R}^{p-1}\right)
$$

and likewise for $L^{2}\left(S^{q-2}\right)$, we have a decomposition of the Hilbert space $L^{2}(C)$ into the discrete direct sum:

$$
L^{2}(C) \simeq \sum_{l, k=0}^{\infty} L^{2}\left(\mathbb{R}_{+}, \frac{1}{2} r^{p+q-5} d r\right) \otimes \mathcal{H}^{l}\left(\mathbb{R}^{p-1}\right) \otimes \mathcal{H}^{k}\left(\mathbb{R}^{q-1}\right) .
$$


Each summand of (4.1.1) is a $K^{\prime}$-isotypic component.

For each $(l, k) \in \mathbb{N}^{2}$, we introduce real analytic function $K_{l, k}(t)$ by

$K_{l, k}(t):= \begin{cases}4(-1)^{l+\frac{p-q}{2}} G_{04}^{20}\left(t^{2} \mid \frac{l+k}{2}, \frac{-q+3+l-k}{2}, \frac{-p-q+6-l-k}{2}, \frac{-p+3-l+k}{2}\right) & \text { Case 1, } \\ 4(-1)^{k} G_{04}^{20}\left(t^{2} \mid \frac{l+k}{2}, \frac{-p+3-l+k}{2}, \frac{-p-q+6-l-k}{2}, \frac{-q+3+l-k}{2}\right) & \text { Case 2. }\end{cases}$

Here, $G_{04}^{20}\left(x \mid b_{1}, b_{2}, b_{3}, b_{4}\right)$ denotes Meijer's $G$-function (see Appendix 7.6 for definition). For the definition of Cases 1 and 2 with regard to the parameter $(l, k) \in \mathbb{N}^{2}$, we recall (3.1.1), namely,

Case 1: $\frac{p-q}{2}+l-k \geq 0$,

Case $2: \frac{p-q}{2}+l-k \leq 0$.

The above formulas give the same result in Cases 1 and 2 if $\frac{p-q}{2}+l-$ $k=0$. Later, we shall give an integral expression of $K_{l, k}$ by means of the Mellin-Barnes type integral (see Lemma 4.5.2). The latter expression is more natural in the sense that the formula is independent of Cases 1 and 2.

Theorem 4.1.1. 1) The unitary inversion operator $\mathcal{F}_{C}=\pi\left(w_{0}\right)$ preserves each summand of (4.1.1), on which $\pi\left(w_{0}\right)$ acts as a form $T_{l, k} \otimes \mathrm{id} \otimes \mathrm{id}$. Here, $T_{l, k}$ is a unitary operator on the Hilbert space $L^{2}\left(\mathbb{R}_{+}, \frac{1}{2} r^{p+q-5} d r\right)$.

2) For each $l, k \in \mathbb{N}$, the unitary operator $T_{l, k}$ is given by the integral transform against the real analytic function $K_{l, k}$ (see (4.1.2) for definition):

$$
\left(T_{l, k} f\right)(r)=\frac{1}{2} \int_{0}^{\infty} K_{l, k}\left(r r^{\prime}\right) f\left(r^{\prime}\right) r^{p+q-5} d r^{\prime} .
$$

Remark 4.1.2 (Case $q=2$ ). Theorem 4.1.1 covers the case $q=2$, where the representation $\pi$ is essentially a highest weight module (see Section 1.7 for the $n_{2}=1$ case). In this case, $\mathcal{H}^{k}\left(\mathbb{R}^{1}\right)$ is non-zero only if $k=0$ or 1 (see Appendix 7.5 for convention). As we saw in Remark 3.1.4, the pair $(l, k)$ belongs to Case 1 for any $l \in \mathbb{N}$ because the inequality $\frac{p-q}{2}+l-k \geq 0$ is implied by $p \geq 6-q=4$. Hence, by the definition (4.1.2) of $K_{l, k}(t)$, we have

$$
\begin{aligned}
& K_{l, 0}(t)=4(-1)^{l+\frac{p-2}{2}} G_{04}^{20}\left(t^{2} \mid \frac{l}{2}, \frac{l+1}{2}, \frac{-p+4-l}{2}, \frac{-p+3-l}{2}\right), \\
& K_{l, 1}(t)=4(-1)^{l+\frac{p-2}{2}} G_{04}^{20}\left(t^{2} \mid \frac{l+1}{2}, \frac{l}{2}, \frac{-p+3-l}{2}, \frac{-p+4-l}{2}\right) .
\end{aligned}
$$

In view of the symmetric property of the G-function:

$$
G_{04}^{20}\left(x \mid b_{1}, b_{2}, b_{3}, b_{4}\right)=G_{04}^{20}\left(x \mid b_{2}, b_{1}, b_{4}, b_{3}\right),
$$


the above formulas show $K_{l, 0}(t)=K_{l, 1}(t)$. Applying the reduction formula (7.6.14) of the $G$-function of the form $G_{04}^{20}\left(x \mid a, a+\frac{1}{2}, b, b+\frac{1}{2}\right)$, we get

$$
K_{l, 0}(t)=K_{l, 1}(t)=4(-1)^{l+\frac{p-2}{2}} t^{-\frac{p-3}{2}} J_{p-3+2 l}(4 \sqrt{t}) .
$$

Thus, the integral transform $T_{l, k}(k=0,1)$ is the Hankel transform on $\mathbb{R}_{+}$ (after a suitable change of variables). Therefore, Theorem 4.1.1 in the case $q=2$ gives the same result with [46. Theorem 6.1.1], but the proof here is different from that of 46$]$.

Remark 4.1.3 (Comparison with the Weil representation). We record here the corresponding result for the Schrödinger model $L^{2}\left(\mathbb{R}^{n}\right)$ of the Weil representation $\varpi$ of $M p(n, \mathbb{R})$ (see also 46 , Remark 6.1.3]). We adopt the same normalization of the Weil representation with [38]. Then, the unitary inversion operator $\varpi\left(w_{0}^{\prime}\right)=e^{\frac{\sqrt{-1} n \pi}{4}} \mathcal{F}_{\mathbb{R}^{n}}$.

1) According to the $O(n)$-isotypic decomposition of $L^{2}\left(\mathbb{R}^{n}\right)$

$$
L^{2}\left(\mathbb{R}^{n}\right) \simeq \sum_{l=0}^{\infty} L^{2}\left(\mathbb{R}_{+}, r^{n-1} d r\right) \otimes \mathcal{H}^{l}\left(\mathbb{R}^{n}\right),
$$

the unitary inversion operator $\varpi\left(w_{0}^{\prime}\right)$ decomposes as

$$
\varpi\left(w_{0}^{\prime}\right) \simeq \sum_{l=0}^{\infty} T_{l}^{\prime} \otimes \mathrm{id},
$$

by a countable family of unitary operators $T_{l}^{\prime}(l \in \mathbb{N})$ on $L^{2}\left(\mathbb{R}_{+}, r^{n-1} d r\right)$.

2) The unitary operator $T_{l}^{\prime}$ is given by the Hankel transform

$$
\left(T_{l}^{\prime} f\right)(r)=\int_{0}^{\infty} K_{l}^{\prime}\left(r r^{\prime}\right) f\left(r^{\prime}\right) r^{\prime n-1} d r^{\prime},
$$

where the kernel $K_{l}^{\prime}(t)$ is defined by

$$
K_{l}^{\prime}(t):=e^{-\frac{\sqrt{-1}(n-1+2 l)}{4} \pi} t^{-\frac{n-2}{2}} J_{\frac{n-2+2 l}{2}}(t) .
$$

Returning to Theorem 4.1.1, we remark that the group law $w_{0}^{2}=1$ in $G$ implies $\pi\left(w_{0}\right)^{2}=\mathrm{id}$, and consequently $T_{l, k}^{2}=$ id for every $l, k \in \mathbb{N}$. Further, as $\pi\left(w_{0}\right)$ is a unitary operator on $L^{2}(C)$, so is its restriction $T_{l, k}$ on $L^{2}\left(\mathbb{R}_{+}, \frac{1}{2} r^{p+q-5} d r\right)$ for every $l, k$. Hence, Theorem 4.1.1 gives a simple group theoretic proof for the Plancherel and reciprocal formulas on the integral transform involving the $G$-functions due to C. Fox [19]: 
Corollary 4.1.4 (Plancherel formula). Let $b_{1}, b_{2}, \gamma$ be half-integers such that $b_{1} \geq 0, \gamma \geq 1, \frac{1-\gamma}{2} \leq b_{2} \leq \frac{1}{2}+b_{1}$. Then the integral transform

$$
S_{b_{1}, b_{2}, \gamma}: f(x) \mapsto \frac{1}{\gamma} \int_{0}^{\infty} G_{04}^{20}\left((x y)^{\frac{1}{\gamma}} \mid b_{1}, b_{2}, 1-\gamma-b_{1}, 1-\gamma-b_{2}\right) f(y) d y
$$

is a unitary operator on $L^{2}\left(\mathbb{R}_{+}\right)$. In particular, we have

$$
\left\|S_{b_{1}, b_{2}, \gamma} f\right\|_{L^{2}\left(\mathbb{R}_{+}\right)}=\|f\|_{L^{2}\left(\mathbb{R}_{+}\right)} .
$$

Proof. Set $b_{1}:=\frac{l+k}{2}, b_{2}:=\frac{-q+3+l-k}{2}, \gamma:=\frac{p+q-4}{2}, x=r^{2 \gamma}, y=r^{2 \gamma}$. Then, the assertion is equivalent to the fact that $T_{l, k}$ is a unitary operator on $L^{2}\left(\mathbb{R}_{+}, \frac{1}{2} r^{p+q-5} d r\right)$.

Corollary 4.1.5 (Reciprocal formula). Retain the notation and the assumption as in Corollary 4.1.4. Then, the unitary operator $S_{b_{1}, b_{2}, \gamma}$ is of order two in $L^{2}\left(\mathbb{R}_{+}\right)$, that is, the following reciprocal formula holds for $f \in L^{2}\left(\mathbb{R}_{+}\right)$:

$$
\begin{aligned}
\left(S_{b_{1}, b_{2}, \gamma} f\right)(x) & =\frac{1}{\gamma} \int_{0}^{\infty} G_{04}^{20}\left((x y)^{\frac{1}{\gamma}} \mid b_{1}, b_{2}, 1-\gamma-b_{1}, 1-\gamma-b_{2}\right) f(y) d y \\
f(x) & =\frac{1}{\gamma} \int_{0}^{\infty} G_{04}^{20}\left((x y)^{\frac{1}{\gamma}} \mid b_{1}, b_{2}, 1-\gamma-b_{1}, 1-\gamma-b_{2}\right)\left(S_{b_{1}, b_{2}, \gamma} f\right)(y) d y
\end{aligned}
$$

Remark 4.1.6 (Fox's reciprocal formula). The reciprocal formula for the $G$-transform was found by C. Fox [19] for the following generality:

$$
G_{2 n, 2 m}^{m, n}\left(x \mid \begin{array}{l}
a_{1}, \cdots, a_{n}, 1-\gamma-a_{1}, \cdots, 1-\gamma-a_{n} \\
b_{1}, \cdots, b_{m}, 1-\gamma-b_{1}, \cdots, 1-\gamma-b_{m}
\end{array}\right) .
$$

Corollary 4.1 .5 is a special case of Fox's formula corresponding to the case $(m, n)=(2,0)$. On the other hand, it follows from Remark 4.1.2 that Corollary 4.1 .5 in the special case when $q=2$ yields the classic reciprocal formula of the Hankel transform ([79, §14.3], see also [46] for the connection with the representation of the conformal group $O(p, 2))$. Our approach gives a new representation theoretic interpretation (and also a proof) of these formulas.

Remark 4.1.7 (Fourth order differential equation). Let $\Omega$ be the Casimir element for the Lie algebra $\mathfrak{k}$. Since an element of the Lie algebra $\mathfrak{n}^{\max }$ acts on smooth vectors of $L^{2}(C)$ as a differential operator of second order (see $(2.3 .16)$ ), the Casimir operator $d \pi(\Omega)$ acts as a differential operator on $C$ of fourth order. Let us examine what information on $K_{l, k}(t)$ we can obtain from the Casimir operator $d \pi(\Omega)$. 
Since $\operatorname{Ad}\left(w_{0}\right) \Omega=\Omega$, we have the commutative relation

$$
\pi\left(w_{0}\right) \circ d \pi(\Omega)=d \pi(\Omega) \circ \pi\left(w_{0}\right) .
$$

On the other hand, $\Omega$ commutes with all the elements in $\mathfrak{k}$, in particular with $\mathfrak{k}^{\prime}$. This implies that the differential operator $d \pi(\Omega)$ preserves each summand of (4.1.1), and the identity (4.1.6) can be regarded as the identity on each summand of (4.1.1). Hence, (4.1.6) leads us to the fact that the kernel function $K_{l, k}(t)$ for $\pi\left(w_{0}\right)$ solves a certain differential equation of order four for each $(l, k)$. In view of the formula (4.1.2) of $K_{l, k}$, this corresponds to the fact that Meijer's G-function $G_{04}^{20}\left(x \mid b_{1}, b_{2}, b_{3}, b_{4}\right)$ satisfies the fourth order differential equation (see (7.6.6)):

$$
\prod_{j=1}^{4}\left(x \frac{d}{d x}-b_{j}\right) u=0 .
$$

For $q=2$, the situation becomes simpler because the minimal representation $\pi$ is essentially a highest weight module. In this case, the Lie algebra $\mathfrak{k}=\mathfrak{s o}(p) \oplus \mathfrak{s o}(q)$ contains the center $\mathfrak{s o}(q)=\mathfrak{s o}(2)$. Taking a normalized generator $Z$ in $\mathfrak{s o}(2)$, we can show

$$
d \pi(\Omega)=d \pi(Z)^{2}+\text { constant } .
$$

We note that the differential operator $d \pi(Z)$ is of order two, and this in turn corresponds to the fact that the kernel function $K_{l, k}$ reduces to the Bessel function (see (4.1.4)) solving the Bessel differential equation of order two (Appendix 7.2).

The rest of this chapter is devoted to the proof of Theorem 4.1.1. The key properties of the element $w_{0}$ and the unitary inversion operator $\pi\left(w_{0}\right)$ that we use in the proof are listed as follows:

1) $w_{0}$ commutes with $K^{\prime}$.

2) $\operatorname{Ad}\left(w_{0}\right) H=-H($ see $(2.5 .2))$.

3) $\left.\pi\left(w_{0}\right)\right|_{H_{l, k}}= \pm$ id (Theorem $\left.3.1 .1(3)\right)$.

The first property (1) gives immediately the proof of Theorem 4.1.1 (1) (see Section 4.2).

The second property (2) implies that the kernel function $K_{l, k}$ of the unitary operator $T_{l, k}$ is a function essentially of one variable $r r^{\prime}$ (see Section 4.4).

The non-trivial part of Theorem 4.1 .1 is to prove that this one variable function is given by Meijer's $G$-function $G_{04}^{20}$, namely, by the formula (4.1.2). 
The property (3) is used for the proof of this part. Our trick here is based on Fact 4.3.1, which assures that the desired formula (4.1.3) holds for any function $f$ once we can prove (4.1.3) holds for a specific function $f=f_{l, k}$ (a $\left(K, K^{\prime}\right)$-skeleton function). Actual computations for this are carried out in Section 4.6. A technical ingredient of the proof is to show that $K_{l, k}$ is a tempered distribution (see Proposition 4.5.6).

Summing up the formulas for $K_{l, k}$ over $l, k$, we shall give a proof of an integral formula of $\pi\left(w_{0}\right)$ for an arbitrary $L^{2}$-function on $C$ in the next Chapter 5 (see Theorem 5.1.1).

\subsection{Proof of Theorem 4.1.1 (1)}

As we have explained at the beginning of Section 4.1, the decomposition (4.1.1) of the Hilbert space $L^{2}(C)$ corresponds to the branching laws of the restriction of the unitary representation $\left(\pi, L^{2}(C)\right)$ from $G$ to $K^{\prime} \simeq$ $O(p-1) \times O(q-1)$. Thanks to the group law $w_{0} m=m w_{0}$ (see 12 in Section 2.5), the relation $\pi\left(w_{0}\right) \circ \pi(m)=\pi(m) \circ \pi\left(w_{0}\right)$ holds for all $m \in K^{\prime}$. Therefore, the unitary inversion operator $\pi\left(w_{0}\right)$ preserves each summand of the decomposition (4.1.1).

We now observe that the group $K^{\prime}$ acts irreducibly on $\mathcal{H}^{l}\left(\mathbb{R}^{p-1}\right) \otimes$ $\mathcal{H}^{k}\left(\mathbb{R}^{q-1}\right)$ (see Appendix 7.5, H2). Therefore, it follows from Schur's lemma that $\pi\left(w_{0}\right)$ acts on each summand:

$$
L^{2}\left(\mathbb{R}_{+}, \frac{1}{2} r^{p+q-5} d r\right) \otimes \mathcal{H}^{l}\left(\mathbb{R}^{p-1}\right) \otimes \mathcal{H}^{k}\left(\mathbb{R}^{q-1}\right)
$$

as the form $T_{l, k} \otimes \mathrm{id} \otimes \mathrm{id}$ for some operator $T_{l, k}$ on $L^{2}\left(\mathbb{R}_{+}, \frac{1}{2} r^{p+q-5} d r\right)$. Such an operator $T_{l, k}$ must be unitary because $\pi\left(w_{0}\right)$ is unitary. Now Theorem 4.1.1 (1) has been proved.

\subsection{Preliminary results on multiplier operators}

We recall the classic theory of multipliers on $\mathbb{R}$, followed by the observation that the multiplier is determined by the action on an (appropriate) single function.

We write $l(t)(t \in \mathbb{R})$ for the translation operator on $L^{2}(\mathbb{R})$, namely,

$$
(l(t) F)(x):=F(x-t) \quad \text { for } F \in L^{2}(\mathbb{R}) .
$$

An operator $B$ on $L^{2}(\mathbb{R})$ is called translation invariant if

$$
B \circ l(t)=l(t) \circ B \quad \text { for any } t \in \mathbb{R} .
$$


We write $\mathcal{S}(\mathbb{R})$ for the space of rapidly decreasing $C^{\infty}$-functions on $\mathbb{R}$ (the Schwartz space endowed with the Fréchet topology), and $\mathcal{S}^{\prime}(\mathbb{R})$ for its dual space consisting of tempered distributions. Then, the Fourier transform $\mathcal{F}: \mathcal{S}(\mathbb{R}) \rightarrow \mathcal{S}(\mathbb{R}), g \mapsto \frac{1}{\sqrt{2 \pi}} \int_{\mathbb{R}} g(x) e^{\sqrt{-1} x \xi} d x$ (see (1.2.1)) extends to $\mathcal{S}^{\prime}(\mathbb{R})$ by

$$
\langle U, \bar{g}\rangle=\langle U, \overline{\mathcal{F} g}\rangle \quad \text { for } U \in \mathcal{S}^{\prime}(\mathbb{R}), g \in \mathcal{S}(\mathbb{R}) .
$$

For $U \in \mathcal{S}^{\prime}(\mathbb{R})$ and $f \in \mathcal{S}(\mathbb{R})$, we have

$$
l(t)(U * f)=U *(l(t) f) \text { for any } t \in \mathbb{R} .
$$

Hence, $f \mapsto U_{*} f$ is translation invariant. Furthermore, if $\mathcal{F} U$ is a bounded measurable function, then $f \mapsto U * f$ extends to a bounded operator on $L^{2}(\mathbb{R})$ and its operator norm is given by $\sqrt{2 \pi}\|\mathcal{F} U\|_{L^{\infty}(\mathbb{R})}$ because

$$
\|U * f\|_{L^{2}(\mathbb{R})}=\|\mathcal{F}(U * f)\|_{L^{2}(\mathbb{R})}=\sqrt{2 \pi}\|(\mathcal{F} U)(\mathcal{F} f)\|_{L^{2}(\mathbb{R})} .
$$

Conversely, the following theorem also holds:

Fact 4.3.1. Let $B$ be a bounded, translation invariant operator on $L^{2}(\mathbb{R})$. Then we have

1) There exists a unique tempered distribution $U$ whose Fourier transform $\mathcal{F} U$ is a bounded measurable function such that $B f=U * f$ for any $f \in L^{2}(\mathbb{R})$.

2) If, moreover, $B f_{0}=f_{1}$ for some function $f_{0} \in L^{2}(\mathbb{R})$ such that $\mathcal{F} f_{0}(\xi) \neq 0$ for a.e. $\xi \in \mathbb{R}$, then $U=\frac{1}{\sqrt{2 \pi}} \mathcal{F}^{-1}\left(\frac{\mathcal{F} f_{1}}{\mathcal{F} f_{0}}\right)$.

Proof. 1) See Stein-Weiss [68, Chapter I, Theorem 3.18], for example.

2) It follows from $B f_{0}=f_{1}$ that $\mathcal{F}\left(U * f_{0}\right)=\mathcal{F} f_{1}$, and therefore we have

$$
\sqrt{2 \pi}(\mathcal{F} U)\left(\mathcal{F} f_{0}\right)=\mathcal{F} f_{1} .
$$

Hence, the bounded measurable function $\mathcal{F} U$ is determined by the formula

$$
\mathcal{F} U(\xi)=\frac{1}{\sqrt{2 \pi}} \frac{\mathcal{F} f_{1}(\xi)}{\mathcal{F} f_{0}(\xi)} .
$$

Next, we introduce two linear maps $\sigma_{+}$and $\sigma_{-}$by

$$
\begin{array}{ll}
\sigma_{+}: L^{2}\left(\mathbb{R}_{+}, \frac{1}{2} r^{p+q-5} d r\right) \rightarrow L^{2}(\mathbb{R}), \quad f(r) \mapsto \frac{1}{\sqrt{2}} e^{\frac{p+q-4}{2} x} f\left(e^{x}\right), \\
\sigma_{-}: L^{2}\left(\mathbb{R}_{+}, \frac{1}{2} r^{p+q-5} d r\right) \rightarrow L^{2}(\mathbb{R}), \quad f(r) \mapsto \frac{1}{\sqrt{2}} e^{-\frac{p+q-4}{2} x} f\left(e^{-x}\right) .
\end{array}
$$


Then, both $\sigma_{+}$and $\sigma_{-}$are unitary operators. Further, clearly we have

$$
\left(\sigma_{-} f\right)(x)=\left(\sigma_{+} f\right)(-x) .
$$

The inverse map $\sigma_{-}^{-1}$ is given by

$$
\left(\sigma_{-}^{-1} F\right)(r)=\sqrt{2} r^{-\frac{p+q-4}{2}} F(-\log r) \quad \text { for } F \in L^{2}(\mathbb{R}) .
$$

We define a subspace $\mathcal{S}$ of $L^{2}\left(\mathbb{R}_{+}, \frac{1}{2} r^{p+q-5} d r\right)$ by

$$
\mathcal{S}:=\sigma_{-}^{-1}(\mathcal{S}(\mathbb{R}))=\sigma_{+}^{-1}(\mathcal{S}(\mathbb{R})),
$$

and endow $\mathcal{S}$ with the topology induced from that of the Schwartz space $\mathcal{S}(\mathbb{R})$. Now let $\mathcal{S}^{\prime}$ be the dual space of $\mathcal{S}$.

Since $\sigma_{-}$is unitary, we get the following identity for $F \in L^{2}(\mathbb{R})$ and $u \in L^{2}\left(\mathbb{R}_{+}, \frac{1}{2} r^{p+q-5} d r\right):$

$$
\begin{aligned}
\left\langle\sigma_{-} u, F\right\rangle_{L^{2}(\mathbb{R})} & =\int_{\mathbb{R}}\left(\sigma_{-} u\right)(x) F(x) d x \\
& =\left\langle u, \sigma_{-}^{-1} F\right\rangle_{L^{2}\left(\mathbb{R}_{+}, \frac{1}{2} r^{p+q-5} d r\right)} .
\end{aligned}
$$

Then $\sigma_{-}$extends naturally to an isomorphism from the dual space $\mathcal{S}^{\prime}$ onto $\mathcal{S}^{\prime}(\mathbb{R})$ by the formula

$$
\left\langle\sigma_{-} u, F\right\rangle:=\left\langle u, \sigma_{-}^{-1} F\right\rangle \quad \text { for } F \in \mathcal{S}(\mathbb{R}), u \in \mathcal{S}^{\prime} .
$$

Recall from (3.1.2) that we have defined a family of real analytic functions $f_{l, k}$ on $\mathbb{R}_{+}$parametrized by $(l, k) \in \mathbb{N}^{2}$. As we saw in Lemma 3.4.1, $f_{l, k} \in L^{2}\left(\mathbb{R}_{+}, \frac{1}{2} r^{p+q-5} d r\right)$.

For a continuous operator $A$ on $L^{2}\left(\mathbb{R}_{+}, \frac{1}{2} r^{p+q-5} d r\right)$, we define a continuous operator $\tilde{A}$ on $L^{2}(\mathbb{R})$ by

$$
\tilde{A}:=\sigma_{-} \circ A \circ \sigma_{+}^{-1} .
$$

Thus, the following diagram commutes:

$$
\begin{array}{ccc}
L^{2}\left(\mathbb{R}_{+}, \frac{1}{2} r^{p+q-5} d r\right) & \stackrel{A}{\longrightarrow} L^{2}\left(\mathbb{R}_{+}, \frac{1}{2} r^{p+q-5} d r\right) \\
\sigma_{+} \downarrow & & \sigma_{-} \downarrow \\
L^{2}(\mathbb{R}) & \stackrel{\tilde{A}}{\longrightarrow} & L^{2}(\mathbb{R}) .
\end{array}
$$

Since $\sigma_{ \pm}$are unitary operators, $A$ is unitary if and only if $\tilde{A}$ is unitary. 
For $\kappa \in \mathcal{S}^{\prime}$, we define an operator $A_{\kappa}$ by

$$
A_{\kappa}: \mathcal{S} \rightarrow \mathcal{S}^{\prime}, \quad f(r) \mapsto \frac{1}{2} \int_{0}^{\infty} \kappa\left(r r^{\prime}\right) f\left(r^{\prime}\right) r^{\prime p+q-5} d r^{\prime} .
$$

It follows from the definition (4.3.3) of $\sigma_{+}$and $\sigma_{-}$that

$$
\left(\sigma_{-} \kappa * \sigma_{+} f\right)(x)=\frac{1}{2} e^{-\frac{p+q-4}{2} x} \int_{-\infty}^{\infty} e^{(p+q-4) y} \kappa\left(e^{-x} e^{y}\right) f\left(e^{y}\right) d y .
$$

Then, by the change of variables, we have

$$
A_{\kappa} f=\frac{1}{\sqrt{2}} \sigma_{-}^{-1}\left(\sigma_{-} \kappa * \sigma_{+} f\right) \quad \text { for } f \in \mathcal{S} .
$$

The following lemma characterizes operators of the form $A_{\kappa}$ :

Lemma 4.3.2. Let $\{\rho(t): t \in \mathbb{R}\}$ be a one parameter family of unitary operators on $L^{2}\left(\mathbb{R}_{+}, \frac{1}{2} r^{p+q-5} d r\right)$ defined by

$$
(\rho(t) f)(r):=e^{-\frac{p+q-4}{2} t} f\left(e^{-t} r\right) .
$$

Suppose that a unitary operator $T$ on $L^{2}\left(\mathbb{R}_{+}, \frac{1}{2} r^{p+q-5} d r\right)$ satisfies the following (anti-)commutative relation:

$$
T \circ \rho(t)=\rho(-t) \circ T \quad \text { for any } t \in \mathbb{R} .
$$

Then, there exists a unique distribution $\kappa \in \mathcal{S}^{\prime}$ such that $T=A_{\kappa}$.

Proof. For a general operator $A$ on $L^{2}\left(\mathbb{R}_{+}, \frac{1}{2} r^{p+q-5} d r\right)$, we shall use the symbol $\tilde{A}$ to denote $\sigma_{-} \circ A \circ \sigma_{+}^{-1}$ as in (4.3.7). Then we have

$$
\begin{aligned}
& \widetilde{T \circ \rho(t)}=\sigma_{-} \circ(T \circ \rho(t)) \circ \sigma_{+}^{-1}=\tilde{T} \circ\left(\sigma_{+} \circ \rho(t) \circ \sigma_{+}^{-1}\right), \\
& \widetilde{\rho(-t) \circ T}=\sigma_{-} \circ(\rho(-t) \circ T) \circ \sigma_{+}^{-1}=\left(\sigma_{-} \circ \rho(-t) \circ \sigma_{-}^{-1}\right) \circ \tilde{T} \text {. }
\end{aligned}
$$

On the other hand, by a simple computation, we have the following identities:

$$
\sigma_{+} \circ \rho(t) \circ \sigma_{+}^{-1}=\sigma_{-} \circ \rho(-t) \circ \sigma_{-}^{-1}=l(t) .
$$

Here, $l(t)$ denotes the translation operator (4.3.1). Hence, the relation (4.3.12) is equivalent to

$$
\tilde{T} \circ l(t)=l(t) \circ \tilde{T} \quad \text { for any } t \in \mathbb{R},
$$


that is, $\tilde{T}$ is a translation invariant bounded operator on $L^{2}(\mathbb{R})$. Therefore, the operator $\tilde{T}$ must be a convolution operator $U *$ for some tempered distribution $U \in \mathcal{S}^{\prime}(\mathbb{R})$ such that its Fourier transform $\mathcal{F} U$ is a bounded function (see Fact 4.3.1 (1)).

Finally, by setting $\kappa:=\sqrt{2} \sigma_{-}^{-1} U$, we have for any $f \in L^{2}\left(\mathbb{R}_{+}, \frac{1}{2} r^{p+q-5} d r\right)$,

$$
\begin{aligned}
T f & =\sigma_{-}^{-1} \circ \tilde{T} \circ \sigma_{+} f \\
& =\frac{1}{\sqrt{2}} \sigma_{-}^{-1}\left(\sigma_{-} \kappa * \sigma_{+} f\right) \\
& =A_{\kappa} f
\end{aligned}
$$

by (4.3.10). Therefore, $T=A_{\kappa}$.

\subsection{Reduction to Fourier analysis}

In this section, we apply the results of the previous section to the unitary operator $T_{l, k}$ on $L^{2}\left(\mathbb{R}_{+}, \frac{1}{2} r^{p+q-5} d r\right)$. The main result of this section is Proposition 4.4.4.

We begin with a refinement of Lemma 3.4.1.

Lemma 4.4.1. $f_{l, k}$ belongs to $\mathcal{S}$. In particular, $f_{l, k} \in L^{2}\left(\mathbb{R}_{+}, \frac{1}{2} r^{p+q-5} d r\right)$.

Proof. By the definition of $\mathcal{S}$ (see (4.3.5)), it is sufficient to show $\sigma_{-} f_{l, k} \in$ $\mathcal{S}(\mathbb{R})$. The proof is divided into two steps.

Step 1: For any $m \in \mathbb{N}, x^{m}\left(\sigma_{-} f_{l, k}\right)(x)$ is rapidly decreasing.

By the definition (3.1.2) of $f_{l, k}$, we have

$$
\left(\sigma_{-} f_{l, k}\right)(x)=\frac{1}{\sqrt{2}} e^{-\left(l+k+\frac{p+q}{2}-2\right) x} \times \begin{cases}\widetilde{K}_{\frac{q-3}{2}+k}\left(2 e^{-x}\right) & \text { Case } 1, \\ \widetilde{K}_{\frac{p-3}{2}+l}\left(2 e^{-x}\right) & \text { Case } 2 .\end{cases}
$$

Therefore, by the asymptotic behavior of $K$-Bessel functions (see Fact 7.2.1 1), 2), respectively), we get

$$
\begin{aligned}
& \left(\sigma_{-} f_{l, k}\right)(x) \sim \frac{e^{-x}}{2 \sqrt{2}} \times\left\{\begin{array}{ll}
\Gamma\left(\frac{q-3}{2}+k\right) e^{-\left(\frac{p-q}{2}+l-k\right) x} & \text { Case 1, } \\
\Gamma\left(\frac{p-3}{2}+l\right) e^{\left(\frac{p-q}{2}+l-k\right) x} & \text { Case 2, }
\end{array} \text { as } x \rightarrow+\infty,\right.
\end{aligned}, \begin{array}{ll}
\left(\sigma_{-} f_{l, k}\right)(x) \sim \frac{1}{\sqrt{2}} \times \begin{cases}e^{-\left(\frac{p-2}{2}+l\right) x} e^{-e^{-x}} & \text { Case 1, } \\
e^{-\left(\frac{q-2}{2}+k\right) x} e^{-e^{-x}} & \text { Case 2, }\end{cases}
\end{array}
$$


Thus, Step 1 is proved.

Step 2: For any $n \in \mathbb{N}, \frac{d^{n}}{d x^{n}}\left(\sigma_{-} f_{l, k}(x)\right)$ is rapidly decreasing.

Let us prove the above statement using induction on $n$. We already know from Step 1 that the above statement is true for any $l, k \in \mathbb{N}$ in the case $n=0$. Now assume that the statement is true for $n$. Then the statement for $n+1$ immediately follows from the next claim. Now Step 2 has been proved, and so has Lemma 4.4.1.

Claim 4.4.2. For any $l, k \in \mathbb{N}$, we have the following recurrence formula:

$$
\frac{d}{d x}\left(\sigma_{-} f_{l, k}\right)=-\left(\frac{p+q-4}{2}+l+k\right) \sigma_{-} f_{l, k}+2 \sigma_{-} f_{l+1, k+1} .
$$

Proof. For simplicity, we rewrite the formula (4.4.1) as

$$
\left(\sigma_{-} f_{l, k}\right)(x)=\frac{1}{\sqrt{2}} e^{-a x} \widetilde{K}_{\nu}\left(2 e^{-x}\right)
$$

where

$$
a=\frac{p+q-4}{2}+l+k, \quad \nu= \begin{cases}\frac{q-3}{2}+k & \text { Case 1, } \\ \frac{p-3}{2}+l & \text { Case 2. }\end{cases}
$$

By the recurrence formula (7.2.1) of the $K$-Bessel function, we have

$$
\begin{aligned}
\frac{d}{d x}\left(\sigma_{-} f_{l, k}\right)(x) & =\frac{1}{\sqrt{2}}\left(-a e^{-a x} \widetilde{K}_{\nu}\left(2 e^{-x}\right)+2 e^{-(a+2) x} \widetilde{K}_{\nu+1}\left(2 e^{-x}\right)\right) \\
& =-a\left(\sigma_{-} f_{l, k}\right)(x)+2\left(\sigma_{-} f_{l+1, k+1}\right)(x) .
\end{aligned}
$$

Here, we have used the fact that $(k, l)$ belongs to Case 1 (i.e. $\frac{p-q}{2}+l-k \geq 0$, see (3.1.1) $)$ if and only if $(k+1, l+1)$ belongs to Case 1 , and likewise for Case 2. Therefore, we have proved Claim 4.4.2.

Remark 4.4.3. The above proof for $f_{l, k} \in \mathcal{S}$ was straightforward from the asymptotic behavior of $f_{l, k}$ and its derivatives. We shall prove in Proposition 4.5.6 that $K_{l, k} \in \mathcal{S}^{\prime}$, namely, $\sigma_{-} K_{l, k} \in \mathcal{S}^{\prime}(\mathbb{R})$. Unlike the above proof for $f_{l, k} \in \mathcal{S}$, this is not obvious from the asymptotic behavior of $K_{l, k}$ (see Remark 4.5.1). In fact, it follows from (4.5.1) that

$$
\begin{aligned}
\limsup _{x \rightarrow-\infty} e^{-\epsilon|x|}\left(\sigma_{-} K_{l, k}\right)(x) & =\limsup _{x \rightarrow-\infty} e^{-\epsilon|x|-\frac{x}{4}}\left(\frac{4}{\sqrt{2 \pi}} \cos \left(4 e^{-\frac{x}{2}}-\frac{2 q-3}{4} \pi\right)\right) \\
& =+\infty
\end{aligned}
$$

if $\epsilon<\frac{1}{4}$. Thus, the asymptotic behavior of $\sigma_{-} K_{l, k}$ is worse than that of any polynomial as $x$ tends to $-\infty$. As a result, our proof for $K_{l, k} \in \mathcal{S}^{\prime}$ is 
more delicate, and is based on an explicit computation of the Fourier integral (see Proposition 4.5.6). We note that $\left(\sigma_{-} K_{l, k}\right)(x)$ decays exponentially as $x$ tends to $+\infty$.

Proposition 4.4.4. Let $T_{l, k}$ be the unitary operator defined in Theorem 4.1.1 (1). Then, there exists uniquely a distribution $\kappa_{l, k} \in \mathcal{S}^{\prime}$ such that $T_{l, k}=A_{\kappa_{l, k}}$ (see (4.3.9) for notation). Namely, we have:

$$
\left(T_{l, k} f\right)(r)=\frac{1}{2} \int_{0}^{\infty} \kappa_{l, k}\left(r r^{\prime}\right) f\left(r^{\prime}\right) r^{\prime p+q-5} d r^{\prime}
$$

Proof of Proposition 4.4.4. We recall from (2.3.12) that the unitary operator $\pi\left(e^{t H}\right)$ on $L^{2}(C)$ can be written by means of the unitary operator $\rho(t)$ (see (4.3.11)) on $L^{2}\left(\mathbb{R}_{+}, \frac{1}{2} r^{p+q-5} d r\right)$ as follows:

$$
\pi\left(e^{t H}\right)(f(r) \phi(\omega) \psi(\eta))=(\rho(t) f)(r) \phi(\omega) \psi(\eta),
$$

where $f \in L^{2}\left(\mathbb{R}_{+}, \frac{1}{2} r^{p+q-5} d r\right), \phi \in \mathcal{H}^{l}\left(\mathbb{R}^{p-1}\right)$, and $\psi \in \mathcal{H}^{k}\left(\mathbb{R}^{q-1}\right)$.

Applying $\pi\left(w_{0}\right)$ to the both sides, together with the definition of $T_{l, k}$ (see Theorem 4.1.1 (1)), we obtain

$$
\pi\left(w_{0}\right) \circ \pi\left(e^{t H}\right)(f(r) \phi(w) \psi(\eta))=\left(T_{l, k} \rho(t) f\right)(r) \phi(w) \psi(\eta) .
$$

Similarly, applying $\pi\left(w_{0}\right)$ followed by $\pi\left(e^{-t H}\right)$, we get

$$
\pi\left(e^{-t H}\right) \circ \pi\left(w_{0}\right)(f(r) \phi(w) \psi(\eta))=\left(\rho(-t) T_{l, k} f\right)(r) \phi(w) \psi(\eta) .
$$

On the other hand, it follows from $\operatorname{Ad}\left(w_{0}\right) H=-H$ (see (2.5.2)) that

$$
w_{0} e^{t H}=e^{-t H} w_{0}
$$

and then we have

$$
\pi\left(w_{0}\right) \circ \pi\left(e^{t H}\right)=\pi\left(e^{-t H}\right) \circ \pi\left(w_{0}\right) .
$$

Therefore,

$$
T_{l, k} \circ \rho(t)=\rho(-t) \circ T_{l, k} .
$$

Now, Proposition 4.4 .4 follows from Lemma 4.3.2. 


\subsection{Kernel function $K_{l, k}$}

We defined a family of real analytic functions $K_{l, k}(t)$ in (4.1.2) by means of Meijer's $G$-function $G_{04}^{20}$. This section studies basic properties of $K_{l, k}(t)$ as a preparation for the proof of Theorem 4.1.1 (2). The main result here is Proposition 4.5.6.

We begin with an asymptotic estimate of $K_{l, k}(t)$.

Lemma 4.5.1 (Asymptotic behavior). $K_{l, k}(t)$ has the following asymptotics as $t$ tends to 0 or $\infty$ :

1) As t tends to 0 ,

$$
\begin{aligned}
& K_{l, k}(t)=\left\{\begin{array}{ll}
O\left(t^{-q+3+l-k}\right) & \text { Case 1 } \\
O\left(t^{-p+3-l+k}\right) & \text { Case 2 }
\end{array} \quad(q>2),\right. \\
& K_{l, k}(t)=O\left(t^{l}\right)
\end{aligned}
$$

2) There are some constants $P_{1}, \cdots, Q_{1}, \cdots$ such that

$$
\begin{aligned}
K_{l, k}(t)= & \frac{4}{\sqrt{2 \pi}} t^{-\frac{2 p+2 q-9}{4}} \cos \left(4 t^{\frac{1}{2}}-\frac{2 q-3}{4} \pi\right)\left(1+P_{1} t^{-1}+P_{2} t^{-2}+\cdots\right) \\
& +t^{-\frac{2 p+2 q-9}{4}} \sin \left(4 t^{\frac{1}{2}}-\frac{2 q-3}{4} \pi\right)\left(Q_{1} t^{-\frac{1}{2}}+Q_{2} t^{-\frac{3}{2}}+\cdots\right),
\end{aligned}
$$

as $t$ tends to $+\infty$.

Proof. Directly obtained from the asymptotic formula of Meijer's $G$-function $G_{04}^{20}\left(x \mid b_{1}, b_{2}, 1-\gamma-b_{1}, 1-\gamma-b_{2}\right)$ given in Lemma 7.6.4 in Appendix.

Next, we give an integral expression of $K_{l, k}(t)(t>0)$, where the integral path $L$ will be taken independently of $l, k \in \mathbb{N}$. We note that the integrands in (4.5.2) and (4.5.3) are the same. The first expression (4.5.2) is convenient in Case 1, and the second expression (4.5.3) is convenient in Case 2 (see Remark 4.5.3).

Lemma 4.5.2. Fix a real number $\gamma>-\frac{p+q-5}{2}$, and let $L$ be a contour that starts at $\gamma-\sqrt{-1} \infty$ and ends at $\gamma+\sqrt{-1} \infty$ and passes the real axis at a point $s_{0}$ satisfying $s_{0}<-\frac{p+q-6}{2}$ (see Figure 4.5.1). (Later, we shall assume also that $-\frac{p+q-4}{2}<s_{0}$ in Chapter 5.) Then, we have

$$
\begin{aligned}
K_{l, k}(t) & =\frac{(-1)^{l+\frac{p-q}{2}}}{\pi \sqrt{-1}} \int_{L} \frac{\Gamma\left(\frac{l+k-s}{2}\right) \Gamma\left(\frac{-q+3+l-k-s}{2}\right)}{\Gamma\left(\frac{p+q-4+l+k+s}{2}\right) \Gamma\left(\frac{p-1+l-k+s}{2}\right)} t^{s} d s \\
& =\frac{(-1)^{k}}{\pi \sqrt{-1}} \int_{L} \frac{\Gamma\left(\frac{l+k-s}{2}\right) \Gamma\left(\frac{-p+3-l+k-s}{2}\right)}{\Gamma\left(\frac{p+q-4+l+k+s}{2}\right) \Gamma\left(\frac{q-1-l+k+s}{2}\right)} t^{s} d s .
\end{aligned}
$$




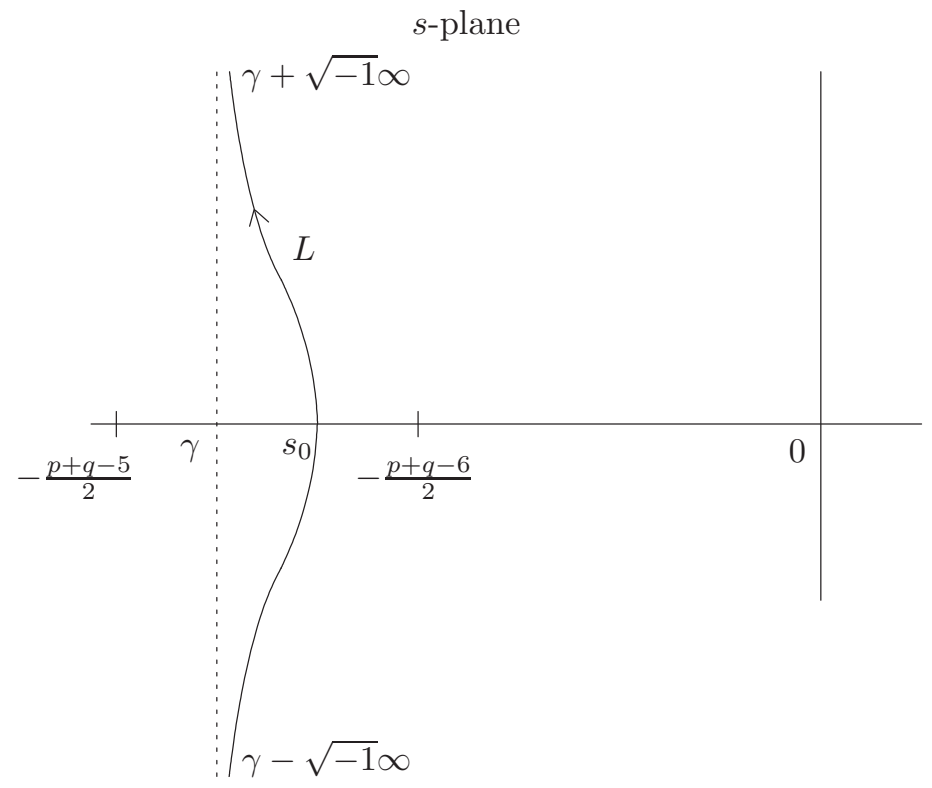

Figure 4.5.1

Proof. The equality $(4.5 .2)=(4.5 .3)$ is an immediate consequence of the following formula:

$$
\frac{\Gamma\left(\frac{-q+3+l-k-s}{2}\right)}{\Gamma\left(\frac{p-1+l-k+s}{2}\right)} \cdot \frac{\Gamma\left(\frac{q-1-l+k+s}{2}\right)}{\Gamma\left(\frac{-p+3-l+k-s}{2}\right)}=(-1)^{\frac{p-q}{2}+l-k},
$$

which is derived from

$$
\Gamma(z) \Gamma(1-z)=\frac{\pi}{\sin \pi z} .
$$

Let us show (4.5.2) in Case 1, and (4.5.3) in Case 2 (see (3.1.1) for the definition of Cases 1 and 2). As meromorphic functions of the variable $s$, the poles of the numerators in the integrands (4.5.2) and (4.5.3) are given by

$$
\begin{aligned}
& W_{1}:=\{l+k+2 a,-q+3+l-k+2 a: a \in \mathbb{N}\}, \\
& W_{2}:=\{l+k+2 a,-p+3-l+k+2 a: a \in \mathbb{N}\},
\end{aligned}
$$

respectively. Then,

$$
\begin{aligned}
& \inf W_{1} \geq-\frac{p+q-6}{2} \quad \text { in Case } 1 \quad \text { (i.e. } \frac{p-q}{2}+l-k \geq 0 \text { ), } \\
& \inf W_{2} \geq-\frac{p+q-6}{2} \quad \text { in Case } 2 \quad \text { (i.e. } \frac{p-q}{2}+l-k \leq 0 \text { ). }
\end{aligned}
$$


Therefore, in either case, the contour $L$ leaves all these sets $W_{1}$ and $W_{2}$ on the right because our $L$ passes the real axis at some point $s_{0}<-\frac{p+q-6}{2}$.

By the definition of Meijer's $G$-function (see (7.6.2) in Appendix, see also Example 7.6.3), we get (4.5.2) in Case 1 and (4.5.3) in Case 2 by the change of variables $s:=2 \lambda$. Hence, Lemma is proved.

Remark 4.5.3. We shall use the expression (4.5.2) in Case 1 and (4.5.3) in Case 2 later. The point here is that there is no cancellation of the poles of the numerator and the denominator of the integrand. For example, the poles of the denominator of the integrand (4.5.2) are given by

$$
V_{1}:=\{-p-q+4-l-k-2 b,-p+1-l+k-2 b: b \in \mathbb{N}\} .
$$

Then,

$$
\sup V_{1}<\inf W_{1} \quad \text { in Case } 1,
$$

and therefore $V_{1} \cap W_{1}=\emptyset$. Similarly, there is no cancellation of the poles of the numerator and the poles of the denominator of the integrand (4.5.3) in Case 2.

As $K_{l, k}$ is a real analytic function on $\mathbb{R}_{+}$, so is $\left(\sigma_{-} K_{l, k}\right)(x)$ on $\mathbb{R}$ (see (4.3.3) for the definition of $\sigma_{-}$), which in turn is a distribution on $\mathbb{R}$. More strongly, we shall see in Proposition 4.5.6 that $\left(\sigma_{-} K_{l, k}\right)(x)$ is a tempered distribution.

For this, we define a meromorphic function $\psi(\zeta)$ on $\mathbb{C}$ by

$$
\begin{aligned}
\psi(\zeta) & :=(-1)^{l+\frac{p-q}{2}} \frac{\Gamma\left(\frac{p+q-4}{4}+\frac{l+k-\sqrt{-1} \zeta}{2}\right) \Gamma\left(\frac{p-q}{4}+\frac{l-k+1-\sqrt{-1} \zeta}{2}\right)}{\Gamma\left(\frac{p+q-4}{4}+\frac{l+k+\sqrt{-1} \zeta}{2}\right) \Gamma\left(\frac{p-q}{4}+\frac{l-k+1+\sqrt{-1} \zeta}{2}\right)} \\
& =(-1)^{k} \frac{\Gamma\left(\frac{p+q-4}{4}+\frac{l+k-\sqrt{-1} \zeta}{2}\right) \Gamma\left(-\frac{p-q}{4}+\frac{-l+k+1-\sqrt{-1} \zeta}{2}\right)}{\Gamma\left(\frac{p+q-4}{4}+\frac{l+k+\sqrt{-1} \zeta}{2}\right) \Gamma\left(-\frac{p-q}{4}+\frac{-l+k+1+\sqrt{-1} \zeta}{2}\right)}
\end{aligned}
$$

We shall use (4.5.5) in Case 1, and (4.5.6) in Case 2. The proof of the equality $(4.5 .5)=(4.5 .6)$ is the same as the proof of the equality $(4.5 .2)=$ (4.5.3).

Lemma 4.5.4. 1) $|\psi(\zeta)|=1$ for $\zeta \in \mathbb{R}$. In particular, the inverse Fourier transform $\mathcal{F}^{-1} \psi$ is defined to be a tempered distribution.

2) $\psi(\zeta)$ is a meromorphic function on $\mathbb{C}$, and the set of its poles is contained in

$$
\{-\sqrt{-1} m: m=1,2,3, \ldots\}
$$


3) For $\eta_{1} \leq \eta \leq \eta_{2}$,

$$
|\psi(\xi-\sqrt{-1} \eta)| \sim\left|\frac{\xi}{2}\right|^{-2 \eta} \quad \text { as }|\xi| \rightarrow \infty .
$$

Proof. 1) Since $\Gamma(\bar{z})=\overline{\Gamma(z)}$ for $z \in \mathbb{C}$, we have $|\psi(\zeta)|=1$. Therefore $\psi \in \mathcal{S}^{\prime}(\mathbb{R})$ and thus $\mathcal{F}^{-1} \psi \in \mathcal{S}^{\prime}(\mathbb{R})$.

2) The proof is straightforward from the definitions (4.5.5) and (4.5.6) in each case.

3) We recall Stirling's asymptotic expansion of the gamma function (see [1, Corollary 1.4.4] for example):

$$
|\Gamma(a+\sqrt{-1} b)|=\sqrt{2 \pi}|b|^{a-\frac{1}{2}} e^{-\frac{\pi|b|}{2}}\left(1+O\left(|b|^{-1}\right)\right)
$$

when $a_{1} \leq a \leq a_{2}$ and $|b| \rightarrow \infty$. Then, for $\alpha \in \mathbb{R}$ and $z=x+\sqrt{-1} y$ $\left(y_{1} \leq y \leq y_{2}\right)$,

$$
\left|\frac{\Gamma(\alpha-\sqrt{-1} z)}{\Gamma(\alpha+\sqrt{-1} z)}\right|=|x|^{2 y}\left(1+O\left(|x|^{-1}\right)\right) \quad \text { as }|x| \rightarrow \infty,
$$

where the constant implied by the Bachmann-Landau symbol $O$ depends only on $\alpha, y_{1}$ and $y_{2}$. Now, applying (4.5.9) to $z=\frac{1}{2}(\xi-\sqrt{-1} \eta)$ twice, we get (4.5.7).

By the change of variable $s=\sqrt{-1} \zeta-\frac{p+q-4}{2}$, the integral formula of $K_{l, k}$ (Lemma 4.5.2) can be restated as follows:

Lemma 4.5.5. Let $\gamma>-\frac{p+q-5}{2}$ and $L^{\prime}$ be an integral path starting from $-\left(\gamma+\frac{p+q-4}{2}\right) \sqrt{-1}-\infty$ and ending at $-\left(\gamma+\frac{p+q-4}{2}\right) \sqrt{-1}+\infty$ passing the imaginary axis at some point in the open interval $\left(-\frac{\sqrt{-1}}{2},-\sqrt{-1}\right)$ (see Figure 4.5.2). Then, we have

$$
K_{l, k}(t)=\frac{1}{\pi} \int_{L^{\prime}} \psi(\zeta) t^{-\frac{p+q-4}{2}+\sqrt{-1} \zeta} d \zeta
$$

or equivalently (see (4.3.3) for the definition of $\sigma_{-}$),

$$
\left(\sigma_{-} K_{l, k}\right)(x)=\frac{1}{\sqrt{2} \pi} \int_{L^{\prime}} \psi(\zeta) e^{-\sqrt{-1} x \zeta} d \zeta
$$




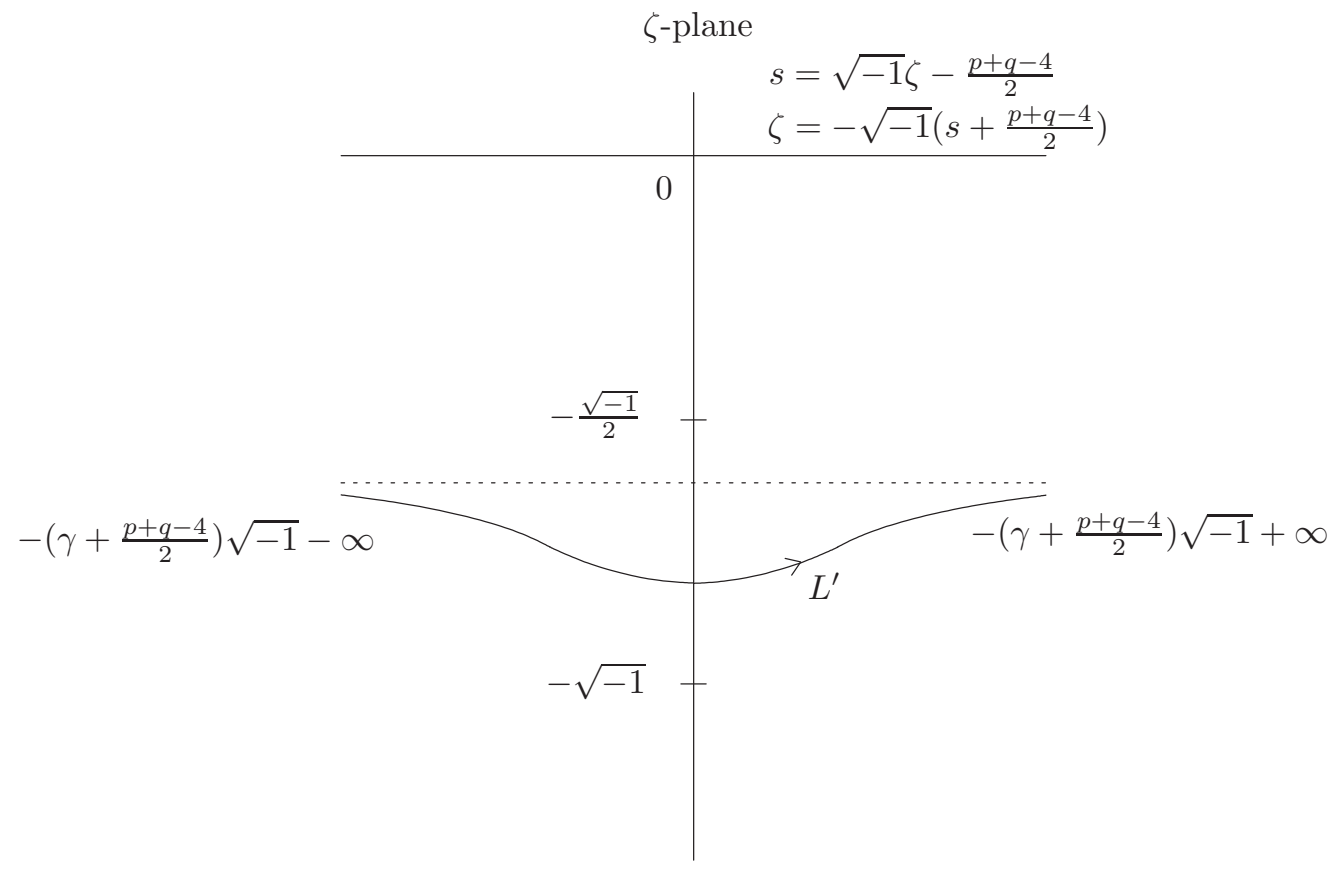

Figure 4.5.2

Now, we recall from Section 4.3 that $\mathcal{S}^{\prime}$ is the dual space of $\mathcal{S}=\sigma_{-}^{-1}(\mathcal{S}(\mathbb{R}))$ via the following diagram:

$$
\begin{array}{ccc}
\mathcal{S}^{\prime} & \stackrel{\sim}{\mathcal{S}^{\prime}(\mathbb{R})} \\
\cup & & \cup \\
\sigma_{-}: L^{2}\left(\mathbb{R}_{+}, \frac{1}{2} r^{p+q-5} d r\right) & \stackrel{\sim}{\rightarrow} & L^{2}(\mathbb{R}) \\
\cup & & \cup \\
\mathcal{S} & \stackrel{\sim}{\rightarrow} & \mathcal{S}(\mathbb{R}) .
\end{array}
$$

Diagram 4.5.3

We are ready to prove the main result of this section:

Proposition 4.5.6. 1) $K_{l, k}$ belongs to $\mathcal{S}^{\prime}$. That is, $\sigma_{-} K_{l, k} \in \mathcal{S}^{\prime}(\mathbb{R})$.

2) The Fourier transform $\mathcal{F}\left(\sigma_{-} K_{l, k}\right)(\zeta)$ of $\sigma_{-} K_{l, k}$ is equal to $\frac{1}{\sqrt{\pi}} \psi(\zeta)$ (see (4.5.5) for definition). In particular, $\left|\mathcal{F}\left(\sigma_{-} K_{l, k}\right)(\zeta)\right|=\frac{1}{\sqrt{\pi}}$ for $\zeta \in \mathbb{R}$.

Proof. It follows from Lemma 4.5.4 (1) that $\psi$ is a tempered distribution, and therefore, its inverse Fourier transform $\mathcal{F}^{-1} \psi \in \mathcal{S}^{\prime}(\mathbb{R})$. We also know 
that $\sigma_{-} K_{l, k} \in C^{\infty}(\mathbb{R})$ by definition. Let $\mathcal{D}^{\prime}(\mathbb{R})$ be the space of distributions, namely, the dual space of $C_{0}^{\infty}(\mathbb{R})$. In light of the inclusion

$$
\mathcal{S}^{\prime}(\mathbb{R}) \subset \mathcal{D}^{\prime}(\mathbb{R}) \supset C^{\infty}(\mathbb{R}),
$$

all the statements of Proposition 4.5.6 will be proved if we show

$$
\sqrt{\pi} \sigma_{-} K_{l, k}=\mathcal{F}^{-1} \psi \quad \text { in } \mathcal{D}^{\prime}(\mathbb{R}),
$$

that is,

$$
\sqrt{\pi} \int_{-\infty}^{\infty}\left(\sigma_{-} K_{l, k}\right)(x) \overline{\phi(x)} d x=\int_{-\infty}^{\infty}\left(\mathcal{F}^{-1} \psi\right)(x) \overline{\phi(x)} d x
$$

holds for any test function $\phi \in C_{0}^{\infty}(\mathbb{R})$. In fact, (4.5.12) will imply that $\sigma_{-} K_{l, k} \in \mathcal{S}^{\prime}(\mathbb{R})$ and $\sqrt{\pi} \mathcal{F}\left(\sigma_{-} K_{l, k}\right)=\psi$ as a tempered distribution.

The key to the proof of (4.5.13) is the integral expression of $K_{l, k}(t)$ stated in Lemma 4.5.5. By (4.5.11), the left-hand side of (4.5.13) amounts to

$$
\begin{aligned}
& \frac{1}{\sqrt{2 \pi}} \int_{-\infty}^{\infty}\left(\int_{L^{\prime}} \psi(\zeta) e^{-\sqrt{-1} x \zeta} d \zeta\right) \overline{\phi(x)} d x \\
= & \frac{1}{\sqrt{2 \pi}} \int_{L^{\prime}} \psi(\zeta) \overline{\left(\int_{-\infty}^{\infty} \phi(x) e^{\sqrt{-1} x \bar{\zeta}} d x\right)} d \zeta \\
= & \int_{L^{\prime}} \psi(\zeta) \overline{(\mathcal{F} \phi)(\bar{\zeta})} d \zeta \\
= & \int_{-\infty}^{\infty} \psi(\zeta) \overline{(\mathcal{F} \phi)(\zeta)} d \zeta \\
= & \text { right-hand side of }(4.5 .13) .
\end{aligned}
$$

In what follows, we explain the above equalities in details:

First equality is by Fubini's theorem. For this justification, take $a>0$ such that $\operatorname{Supp} \phi \subset[-a, a]$. Then,

$$
\left|\overline{\phi(x)} e^{-\sqrt{-1} x \zeta}\right| \leq\|\phi\|_{\infty} e^{a \eta}
$$

for $\zeta=\xi-\sqrt{-1} \eta$ with $\eta>0$. Here, $\|\phi\|_{\infty}$ denotes the $L^{\infty}$ norm.

Since $\gamma>-\frac{p+q-5}{2}$, we may assume $\zeta=\xi-\sqrt{-1} \eta \in L^{\prime}$ satisfies

$$
\eta_{1} \leq \eta \leq \eta_{2}
$$

for some constants $\eta_{1}$ and $\eta_{2}$ such that $\eta_{1}>\frac{1}{2}$ if $|\xi|$ is sufficiently large. Then, there exists a constant $C>0$ such that $|\psi(\zeta)| \leq C|\xi|^{-2 \eta}$ as $|\xi| \rightarrow \infty$, by Lemma 4.5 .4 (3). Thus, if $|\xi|$ is sufficiently large, we have

$$
\left|\psi(\zeta) \overline{\phi(x)} e^{-\sqrt{-1} x \zeta}\right| \leq C\|\phi\|_{\infty}|\xi|^{-2 \eta_{1}} .
$$


Hence, $\psi(\zeta) \overline{\phi(x)} e^{-\sqrt{-1} x \zeta}$ is absolutely integrable on $L^{\prime} \times[-a, a]$ (and therefore, on $\left.L^{\prime} \times(-\infty, \infty)\right)$. Thus, we can apply Fubini's theorem.

Second equality follows immediately from the definition (1.2.1) of the Fourier transform.

Third equality follows from Cauchy's integral formula. First, we observe that $\psi(\zeta) \overline{(\mathcal{F} \phi)(\bar{\zeta})}$ is holomorphic in the domain between the two integral paths $(-\infty, \infty)$ and $L^{\prime}$ since its poles lie only on $\{-\sqrt{-1} m: m=1,2, \cdots\}$ (see Lemma 4.5.4(2)).

Next, let us show

$$
\lim _{|\xi| \rightarrow \infty} \int_{0}^{\eta_{0}}|\psi(\xi-\sqrt{-1} \eta) \overline{(\mathcal{F} \phi)(\xi+\sqrt{-1} \eta)}| d \eta=0
$$

for a fixed $\eta_{0}\left(\geq \gamma+\frac{p+q-4}{2}\right)$ (see Figure 4.5.2). To see (4.5.14), take $a>0$ as before such that $\operatorname{Supp} \phi \subset[-a, a]$. Then, by the Paley-Wiener theorem, there exists a constant $C$ such that $|\overline{\mathcal{F} \phi(\xi+\sqrt{-1} \eta)}| \leq C e^{a \eta}$. Now combining this with Lemma 4.5.4 (3), we get

$$
|\psi(\zeta) \overline{\mathcal{F} \phi(\bar{\zeta})}| \leq C^{\prime}|\xi|^{-2 \eta} \quad \text { as }|\xi| \rightarrow \infty
$$

for $\zeta=\xi-\sqrt{-1} \eta$ and bounded $\eta$. Hence, (4.5.14) is proved. By Cauchy's integral formula, we get the third equality.

Last equality is by the definition of the Fourier transform for tempered distributions:

$$
(f, g)=\left(\mathcal{F}^{-1} f, \mathcal{F}^{-1} g\right) \quad f \in \mathcal{S}^{\prime}(\mathbb{R}), g \in \mathcal{S}(\mathbb{R}) .
$$

Hence, we have proved (4.5.12). Now, the proof of Proposition 4.5.6 is completed.

\subsection{Proof of Theorem 4.1.1 (2)}

In this section, we complete the proof of Theorem 4.1.1 (2). For this, it is sufficient to show the following proposition:

Proposition 4.6.1. $\kappa_{l, k}=K_{l, k}$.

Here, we recall that the kernel distribution $\kappa_{l, k}$ of $T_{l, k}$ is given in Proposition 4.4.4 and that $K_{l, k}$ is defined in (4.1.2).

Proof. The proof makes use of the following: 
Lemma 4.6.2. Let $\kappa_{1}, \kappa_{2} \in \mathcal{S}^{\prime}$. If there exists $\phi \in \mathcal{S}$ such that

$$
\begin{aligned}
& \mathcal{F}\left(\sigma_{+} \phi\right)(\zeta) \neq 0 \quad \text { for any } \zeta \in \mathbb{R}, \\
& A_{\kappa_{1}} \phi=A_{\kappa_{2}} \phi
\end{aligned}
$$

then $\kappa_{1}=\kappa_{2}$. Here, we recall from (4.3.9) for the definition of $A_{\kappa}$.

Proof of Lemma 4.6.2. The identity (4.6.2) implies

$$
\sigma_{-} \kappa_{1} * \sigma_{+} \phi=\sigma_{-} \kappa_{2} * \sigma_{+} \phi
$$

by the formula (4.3.10) of $A_{\kappa}$. Therefore, we have an identity

$$
\mathcal{F}\left(\sigma_{-} \kappa_{1}\right)(\zeta) \cdot \mathcal{F}\left(\sigma_{+} \phi\right)(\zeta)=\mathcal{F}\left(\sigma_{-} \kappa_{2}\right)(\zeta) \cdot \mathcal{F}\left(\sigma_{+} \phi\right)(\zeta)
$$

in $\mathcal{S}^{\prime}(\mathbb{R})$ by taking their Fourier transforms.

On the other hand, it follows from the assumption that $\sigma_{+} \phi \in \mathcal{S}(\mathbb{R})$ and its Fourier transform $\mathcal{F}\left(\sigma_{+} \phi\right)$ does not vanish on $\mathbb{R}$, we can divide the above identity by $\mathcal{F}\left(\sigma_{+} \phi\right)(\zeta)$, and obtain the following identity in $\mathcal{S}^{\prime}(\mathbb{R})$ :

$$
\mathcal{F}\left(\sigma_{-} \kappa_{1}\right)(\zeta)=\mathcal{F}\left(\sigma_{-} \kappa_{2}\right)(\zeta)
$$

Hence, $\sigma_{-} \kappa_{1}=\sigma_{-} \kappa_{2}$, and in turn, $\kappa_{1}=\kappa_{2}$.

We want to apply Lemma 4.6 .2 with $\kappa_{1}:=\kappa_{l, k}, \kappa_{2}:=K_{l, k}, \phi:=f_{l, k}$ (see (3.1.2) for the definition). The conditions in the lemma will be verified by the following steps.

Step 1. $\kappa_{l, k}, K_{l, k} \in \mathcal{S}^{\prime}$. These statements have been already proved in Propositions 4.4 .4 and 4.5.6.

Step 2. $f_{l, k} \in \mathcal{S}$. This has been proved in Lemma 4.4.1.

Step 3. $\mathcal{F}\left(\sigma_{+} f_{l, k}\right)(\zeta)$ has no zero points on $\mathbb{R}$. This statement will follow readily from Claim 4.6.3. We note that we have assumed $p \geq q \geq 2$ and $p+q \geq 6$.

Claim 4.6.3. We recall from (3.1.1) the definitions of Cases 1 and 2. Then,

$$
\begin{aligned}
\mathcal{F}\left(\sigma_{+} f_{l, k}\right)(\zeta)= & \frac{1}{8 \sqrt{\pi}} \Gamma\left(\frac{p+q-4}{4}+\frac{l+k+\sqrt{-1} \zeta}{2}\right) \\
& \times \begin{cases}\Gamma\left(\frac{p-q}{4}+\frac{l-k+1+\sqrt{-1} \zeta}{2}\right) & \text { Case 1, } \\
\Gamma\left(-\frac{p-q}{4}+\frac{-l+k+1+\sqrt{-1} \zeta}{2}\right) & \text { Case 2. }\end{cases}
\end{aligned}
$$


Proof. By the definition (3.1.2) of $f_{l, k}$ and the definition (4.3.3) of $\sigma_{+}$, we have

$$
\left(\sigma_{+} f_{l, k}\right)(x)=\frac{1}{\sqrt{2}} e^{\left(l+k+\frac{p+q}{2}-2\right) x} \times \begin{cases}\widetilde{K}_{\frac{q-3}{2}+k}\left(2 e^{x}\right) & \text { Case 1, } \\ \widetilde{K}_{\frac{p-3}{2}+l}\left(2 e^{x}\right) & \text { Case 2. }\end{cases}
$$

In Case 1, we have

$$
\begin{aligned}
\mathcal{F}\left(\sigma_{+} f_{l, k}\right)(\zeta) & =\frac{1}{\sqrt{2 \pi} \sqrt{2}} \int_{-\infty}^{\infty} e^{\left(l+k+\frac{p+q}{2}-2\right) x} \widetilde{K}_{\frac{q-3}{2}+k}\left(2 e^{x}\right) e^{\sqrt{-1} x \zeta} d x \\
& =\frac{1}{2 \sqrt{\pi}} \int_{0}^{\infty} r^{\left(l+k+\frac{p+q}{2}-3+\sqrt{-1} \zeta\right)} \widetilde{K}_{\frac{q-3}{2}+k}(2 r) d r .
\end{aligned}
$$

Applying the formula (7.2.11) of the Mellin transform for the $K$-Bessel function, we obtain the right-hand side of (4.6.3). Likewise, in Case 2, $\mathcal{F}\left(\sigma_{+} f_{l, k}\right)(\zeta)$ is equal to

$$
\frac{1}{\sqrt{2 \pi} \sqrt{2}} \int_{-\infty}^{\infty} e^{\left(l+k+\frac{p+q}{2}-2\right) x} \widetilde{K}_{\frac{p-3}{2}+l}\left(2 e^{x}\right) e^{\sqrt{-1} x \zeta} d x .
$$

Switching the role of $(p, l)$ and $(q, k)$, we see (4.6.3) holds also in Case 2.

Step 4. $A_{\kappa_{l, k}} f_{l, k}=A_{K_{l, k}} f_{l, k}$.

To see this, we prepare the following explicit formulas. As we shall see below, the proof of (1) is algebraic by using the fact that $\pi\left(w_{0}\right)$ acts on each $K$-type as \pm id. On the other hand, the proof of (2) is based on an explicit integral computation.

Claim 4.6.4. Let $a(l, k)$ be as in (3.1.5).

1) $A_{\kappa_{l, k}} f_{l, k}=(-1)^{a(l, k)+\frac{p-q}{2}} f_{l, k}$.

2) $A_{K_{l, k}} f_{l, k}=(-1)^{a(l, k)+\frac{p-q}{2}} f_{l, k}$.

Proof of Claim 4.6.4. 1) The function $f_{l, k}$ belongs to the $K^{\prime}$-invariant subspace $H_{l, k}$ (see (3.1.4)), and therefore, by Theorem 3.1.1 (3), we have

$$
\pi\left(w_{0}\right)\left(f_{l, k} \phi \psi\right)=(-1)^{a(l, k)+\frac{p-q}{2}} f_{l, k} \phi \psi
$$

for $\phi \in \mathcal{H}^{l}\left(\mathbb{R}^{p-1}\right)$ and $\psi \in \mathcal{H}^{k}\left(\mathbb{R}^{q-1}\right)$. In light of the definition of $T_{l, k}$ (see Theorem 4.1.1 (1)), this implies

$$
T_{l, k} f_{l, k}=(-1)^{a(l, k)+\frac{p-q}{2}} f_{l, k} .
$$

By the definition of $A_{\kappa_{l, k}}$ (see Proposition 4.4.4), the first statement follows. 
2) First, we treat Case 1, namely, the case $\frac{p-q}{2}+l-k \geq 0$. Then,

$$
\begin{aligned}
& \left(A_{K_{l, k}} f_{l, k}\right)(r)=\frac{1}{2 \pi} \int_{0}^{\infty}\left(\int_{L^{\prime}} \psi(\zeta)\left(r r^{\prime}\right)^{-\frac{p+q-4}{2}+\sqrt{-1} \zeta} d \zeta\right) f_{l, k}\left(r^{\prime}\right) r^{\prime p+q-5} d r^{\prime} \\
= & \frac{1}{2 \pi} \int_{L^{\prime}} r^{-\frac{p+q-4}{2}}+\sqrt{-1} \zeta\left(\int_{0}^{\infty} r^{\frac{p+q-6}{2}+\sqrt{-1} \zeta} f_{l, k}\left(r^{\prime}\right) d r^{\prime}\right) \psi(\zeta) d \zeta \\
= & \frac{(-1)^{l+\frac{p-q}{2}}}{8 \pi} \int_{L^{\prime}} \Gamma\left(\frac{p+q-4}{4}+\frac{l+k-\sqrt{-1} \zeta}{2}\right) \\
& \times \Gamma\left(\frac{p-q}{4}+\frac{l-k+1-\sqrt{-1} \zeta}{2}\right) r^{-\frac{p+q-4}{2}+\sqrt{-1} \zeta} d \zeta \\
= & \frac{(-1)^{l+\frac{p-q}{2}}}{8 \pi \sqrt{-1}} \int_{L} \Gamma\left(\frac{l+k-s}{2}\right) \Gamma\left(\frac{-q+3+l-k-s}{2}\right) r^{s} d s \\
= & \frac{1}{2}(-1)^{l+\frac{p-q}{2}} G_{02}^{20}\left(r^{2} \mid \frac{l+k}{2}, \frac{-q+3+l-k}{2}\right) \\
= & (-1)^{l+\frac{p-q}{2}} r^{-\frac{q-3}{2}+l} K_{\frac{q-3}{2}+k}(2 r) \\
= & (-1)^{a(l, k)+\frac{p-q}{2}} f_{l, k}(r) .
\end{aligned}
$$

Let us explain the above equalities in more details.

First equality. This is by the integral expression of $K_{l, k}$ (see Lemma 4.5.5) and the definition (4.3.9) of $A_{K_{l, k}}$.

Second equality. We recall the upper estimate of $|\psi(\zeta)|$ given in Lemma 4.5 .4 (3) and the asymptotic behavior of $f_{l, k}\left(r^{\prime}\right)$ (see (3.4.1) and (3.4.2)). Then, in light of

$$
\left|\psi(\zeta) r^{\prime \frac{p+q-6}{2}+\sqrt{-1} \zeta} f_{l, k}\left(r^{\prime}\right)\right| \leq|\psi(\zeta)| r^{\prime \frac{p+q-6}{2}+\eta}\left|f_{l, k}\left(r^{\prime}\right)\right| \quad \text { for } \zeta=\xi-\sqrt{-1} \eta,
$$

the second equality follows from Fubini's theorem.

Third equality is by Lemma 3.4.3.

Fourth equality is from the change of the variable as before: $s=$ $\sqrt{-1} \zeta-\frac{p+q-4}{2}$.

Fifth equality. The poles of the integrand in (4.6.4) are of the form $l+k+2 a(a \in \mathbb{N})$ or $-q+3+l-k+2 a(a \in \mathbb{N})$. These lie on the right of the contour $L$ because of the assumption $\frac{p-q}{2}+l-k \geq 0$. Hence, the fifth equality holds by the integral expression of Meijer's $G$-function (see (7.6.2) in Appendix).

Sixth equality follows from the reduction formula of the $G$-function (see $(7.6 .13))$.

Seventh equality is by the definition (3.1.2) of $f_{l, k}$ and the definition (3.1.5) of $a(l, k)$. 
Case 2 can be treated in the same manner. In this case, the integral

$$
\int_{L} \Gamma\left(\frac{l+k-s}{2}\right) \Gamma\left(\frac{-p+3-l+k-s}{2}\right) r^{s} d s
$$

arises instead of (4.6.4). But again, by the assumption $\frac{p-q}{2}+l-k \leq 0$, this defines the $G$-function which reduces to $f_{l, k}$ by the same reduction formula.

Step 5. $\kappa_{l, k}=K_{l, k}$.

Steps 3 and 4 imply $\kappa_{l, k}=K_{l, k}$ by Lemma 4.6.2. Thus, Proposition 4.5 .6 is proved.

Now the proof of Theorem 4.1.1 finishes. 


\section{Chapter 5}

\section{Main theorem}

This chapter is a highlight of this book. We find an explicit formula for the 'Fourier transform' $\mathcal{F}_{C}$ on the isotropic cone, in other words, we find an integral kernel for the unitary inversion operator $\pi\left(w_{0}\right)$ on the Schrödinger model $L^{2}(C)$ of the minimal representation. The main result is Theorem 5.1.1.

\subsection{Result of this chapter}

Let $C$ be the conical variety $\left\{x \in \mathbb{R}^{p+q-2} \backslash\{0\}: Q(x)=0\right\}$ where $Q(x)=$ $x_{1}^{2}+\cdots+x_{p-1}^{2}-x_{p}^{2}-\cdots-x_{p+q-2}^{2}$. We recall from the Introduction that the generalized function $K\left(x, x^{\prime}\right)$ on $C \times C$ is defined by the following formula:

$$
K\left(x, x^{\prime}\right) \equiv K\left(p, q ; x, x^{\prime}\right):=c_{p, q} \Phi_{p, q}\left(\left\langle x, x^{\prime}\right\rangle\right),
$$

where $\langle\cdot, \cdot\rangle$ stands for the standard (positive definite) inner product on $\mathbb{R}^{p+q-2}$. The constant $c_{p, q}$ and the Bessel distribution $\Phi_{p, q}(t)$ are given as follows (see (1.3.1), (1.3.2), and (1.3.3)):

$$
\begin{aligned}
& c_{p, q}:= \frac{2(-1)^{\frac{(p-1)(p+2)}{2}}}{\pi^{\frac{p+q-4}{2}},} \\
& \Phi_{p, q}(t):= \begin{cases}\Phi_{\frac{p+q-6}{2}}^{+}(t) & \text { if } \min (p, q)=2, \\
\Psi_{\frac{p+q-6}{2}}^{+}(t) & \text { if } p, q>2 \text { are both even, } \\
\Psi_{\frac{p+q-6}{2}}^{+}(t) & \text { if } p, q>2 \text { are both odd. }\end{cases}
\end{aligned}
$$

We are ready to state the explicit formula for the unitary inversion operator: 
Theorem 5.1.1 (Integral formula for the unitary inversion operator). Let $\left(\pi, L^{2}(C)\right)$ be the Schrödinger model of the minimal representation of $G=O(p, q)$ for $p, q \geq 2$ and $p+q \geq 6$ even, and $w_{0}=\left(\begin{array}{cc}I_{p} & 0 \\ 0 & -I_{q}\end{array}\right)$. Then the unitary operator $\pi\left(w_{0}\right): L^{2}(C) \rightarrow L^{2}(C)$ is given by the following integrodifferential operator:

$$
\pi\left(w_{0}\right) u(x)=\int_{C} K\left(x, x^{\prime}\right) u\left(x^{\prime}\right) d \mu\left(x^{\prime}\right), \quad u \in L^{2}(C) .
$$

The right-hand side of (5.1.4) involves a singular integral. It factors through the Radon transform (see Section 5.2), and we shall see that the right-hand side of (5.1.4) is well-defined for any compactly supported smooth function $u$ on $C$, and extends as a unitary operator on $L^{2}(C)$.

As for the Bessel distribution $\Phi_{p, q}$, we shall give a Mellin-Barnes type integral formula for $\Phi_{p, q}$ in Section $\underline{6.2}$, and the differential equation satisfied by $\Phi_{p, q}$ in Section 6.3 .

Since the action of the maximal parabolic subgroup $\overline{P^{\max }}$ on $L^{2}(C)$ is of a simple form (see $(2.3 .10)-(2.3 .13)$ ), Theorem 5.1 .1 gives an explicit action of the whole group $G$ on $L^{2}(C)$ because $G=\overline{P^{\max }} \amalg \overline{P^{\max }} w_{0} \overline{P^{\max }}$.

Theorem 5.1.1 immediately yields two corollaries about the Plancherel formula and the reciprocal formula of our integral transform.

Corollary 5.1.2 (Plancherel formula). Let $\mathcal{F}_{C}: L^{2}(C) \rightarrow L^{2}(C)$ be an integral transform against the kernel $K\left(x, x^{\prime}\right)$ (see (5.1.1) ), namely,

$$
\left(\mathcal{F}_{C} u\right)(x):=\int_{C} K\left(x, x^{\prime}\right) u\left(x^{\prime}\right) d \mu\left(x^{\prime}\right) .
$$

Then $\mathcal{F}_{C}$ is unitary:

$$
\left\|\mathcal{F}_{C} u\right\|_{L^{2}(C)}=\|u\|_{L^{2}(C)} .
$$

Since the group law $w_{0}^{2}=1$ in $O(p, q)$ implies $\pi\left(w_{0}\right)^{2}=$ id on $L^{2}(C)$, we immediately obtain the following corollary to Theorem 5.1.1, which can also be viewed as giving the inversion formula $\mathcal{F}_{C}^{-1}=\mathcal{F}_{C}$.

Corollary 5.1.3 (Reciprocal formula). Retain the notation as in Corollary 5.1.2. The unitary operator $\mathcal{F}_{C}$ is of order two in $L^{2}(C)$. Namely, we have the following reciprocal relation:

$$
\begin{aligned}
\left(\mathcal{F}_{C} u\right)(x) & =\int_{C} K\left(x, x^{\prime}\right) u\left(x^{\prime}\right) d \mu\left(x^{\prime}\right), \\
u(x) & =\int_{C} K\left(x, x^{\prime}\right)\left(\mathcal{F}_{C} u\right)\left(x^{\prime}\right) d \mu\left(x^{\prime}\right) .
\end{aligned}
$$


Remark 5.1.4 (Comparison with the Schrödinger model of the Weil representation, see Section 1.6). In the case of the Schrödinger model of the Segal-Shale-Weil representation $\varpi$ of the metaplectic group $M p(n, \mathbb{R})$, the corresponding 'inversion' element $w_{0}^{\prime}$ acts on $L^{2}\left(\mathbb{R}^{n}\right)$ as $e^{\frac{\sqrt{-1} n \pi}{4}} \mathcal{F}_{\mathbb{R}^{n}}$, where $\mathcal{F}_{\mathbb{R}^{n}}$ denotes the Fourier transform. We note that $\left(w_{0}^{\prime}\right)^{4}$ gives the unique non-trivial element $\xi_{0}$ in the kernel of the metaplectic covering $M p(n, \mathbb{R}) \rightarrow$ $S p(n, \mathbb{R})$, and $\varpi\left(\xi_{0}\right)=-\mathrm{id}$. This fact reflects the identity $\mathcal{F}_{\mathbb{R}^{n}}^{4}=\mathrm{id}$ on $L^{2}\left(\mathbb{R}^{n}\right)$. Thus, the above two corollaries can be interpreted as the counterparts to the Plancherel formula and the equality $\mathcal{F}_{\mathbb{R}^{n}}^{4}=$ id of the Fourier transform $\mathcal{F}_{\mathbb{R}^{n}}$ on $\mathbb{R}^{n}$.

Remark 5.1.5. In $\sqrt{46}$, Corollary 6.3.1], we gave a different proof of the same Plancherel and reciprocal formulas in the case $q=2$ based on analytic continuation of holomorphic semigroup of operators.

This chapter is organized as follows. In Section 5.2 , we analyze the integral transform (5.1.4) by means of the (singular) Radon transform. In particular, we prove that the integral transform (5.1.4) is well-defined for $u \in C_{0}^{\infty}(C)$ in the sense of distributions. The second step of the proof of Theorem 5.1.1 is to use the restriction from $G$ to $K^{\prime}=K \cap M_{+}^{\max }$ (see Section 2.3) and is to show the $(l, k)$-th spectrum of the unitary inversion operator $\mathcal{F}_{C}$ coincides with the radial part $T_{l, k}$ of $\mathcal{F}_{C}$ when restricted to each $K^{\prime}$-isotypic component $\mathcal{H}^{l}\left(\mathbb{R}^{p-1}\right) \otimes \mathcal{H}^{k}\left(\mathbb{R}^{q-1}\right)$ (see Lemma 5.4.1). The latter operator $T_{l, k}$ was studied in details in the previous chapter (see Theorem 4.1.1). Section 5.3 explains a general formula giving spectra of a $K^{\prime}$-intertwining integral operator. In Section 5.4, we reduce the proof of Theorem 5.1 .1 to the identity of spectra for specific $K^{\prime}$-intertwining integral operators. This identity is verified in Sections 5.5 and 5.6. Some technical parts (e.g. Mellin-Barnes type integral formulas of Bessel distributions) will be postponed until Chapters 6 and 7 .

\subsection{Radon transform for the isotropic cone $C$}

This section studies the kernel $K\left(x, x^{\prime}\right)$. The subtle point in defining $K\left(x, x^{\prime}\right)$ consists of the following two facts:

1) The distribution $\Phi_{p, q}(t)$ is not locally integrable near $t=0$.

2) The level set $\left\langle x, x^{\prime}\right\rangle=t$ is not a regular submanifold in $C \times C$ if $t=0$. 
In fact, the distribution $\Phi_{p, q}(t)$ involves a linear combination of distributions $\delta^{(k-1)}(t)$ and $t^{-k}\left(k=1,2, \ldots, \frac{p+q-6}{2}\right)$ as we shall see in Section 6.2 on the one hand, and the differential form

$$
d Q(x) \wedge d Q\left(x^{\prime}\right) \wedge d\left(\left\langle x, x^{\prime}\right\rangle-t\right)
$$

of $x, x^{\prime}$ vanishes if $\left(x, x^{\prime}\right)$ belongs to the submanifold

$$
Y:=\left\{\left(x, x^{\prime}\right) \in C \times C: \mathbb{R} x=\mathbb{R}\left(I_{p, q} x^{\prime}\right)\right\}
$$

on the other hand. Here, we note that $Y \subset\left\{\left(x, x^{\prime}\right):\left\langle x, x^{\prime}\right\rangle=0\right\}$.

Our idea to give a rigorous definition of $K\left(x, x^{\prime}\right)$ is to factorize the transform (5.1.4) by using the singular Radon transform and then to find a PaleyWiner type theorem of the singular Radon transform.

Let $\delta$ denote the Dirac delta function of one variable. The Radon transform of a function $\varphi$ on $\mathbb{R}^{p+q-2}$ is defined by the formula (see for example [25, Chapter I]):

$$
(R \varphi)(x, t):=\int_{\mathbb{R}^{p+q-2}} \varphi\left(x^{\prime}\right) \delta\left(t-\left\langle x, x^{\prime}\right\rangle\right) d x^{\prime},
$$

for $x \in \mathbb{R}^{p+q-2} \backslash\{0\}, t \in \mathbb{R}$.

The Radon transform $R \varphi$ is well-defined, for example, for a compactly supported continuous function $\varphi$. More generally, $R \varphi$ makes sense if $\varphi$ is a compactly supported distribution such that the multiplication of two distributions $\varphi\left(x^{\prime}\right)$ and $\delta\left(t-\left\langle x, x^{\prime}\right\rangle\right)$ makes sense.

Now we recall the injective map (see (2.2.6))

$$
T: L^{2}(C) \rightarrow \mathcal{S}^{\prime}\left(\mathbb{R}^{p+q-2}\right), \quad u \mapsto u \delta(Q)
$$

yields a compactly supported distribution $T u$ if $u \in C_{0}^{\infty}(C)$. In this context, what we need here is the following result:

Let $C_{0}^{k}(\mathbb{R})$ denote the space of compactly supported functions on $\mathbb{R}$ with continuous derivatives up to $k$.

Lemma 5.2.1. Suppose $u \in C_{0}^{\infty}(C)$.

$0)$ The Radon transform $\mathcal{R} u(x, t):=R(T u)(x, t)$ is well-defined and continuous as a function of $(x, t) \in C \times(\mathbb{R} \backslash\{0\})$. Moreover, there exists $A>0$ such that

$$
\operatorname{Supp} \mathcal{R} u \subset\{(x, t) \in C \times(\mathbb{R} \backslash\{0\}): t \leq A|x|\},
$$

where $|x|:=\left(x_{1}^{2}+\cdots+x_{p+q-2}^{2}\right)^{\frac{1}{2}}$. In particular, $\mathcal{R} u(x, t)$ vanishes if $|t|$ is sufficiently large for a fixed $x \in C$. 
1) If $p, q>2$ and $p+q \geq 8$, then $\mathcal{R} u(x, t)$ extends continuously to $t=0$ and $\mathcal{R} u(x, \cdot) \in C_{0}^{k}(\mathbb{R})$ where $k:=\frac{p+q-8}{2}$.

2) If $\min (p, q)=2$, then $\mathcal{R} u(x, t)$ is bounded on $C \times(\mathbb{R} \backslash\{0\})$.

3) If $p, q>2$ and $p+q=6$ (namely, $(p, q)=(3,3)$ ), then there exists $C \equiv C(x)>0$ such that

$$
|R(T u)(x, t)| \leq C|\log | t||
$$

if $t$ is sufficiently small.

Proof. See [54].

We note that

$$
\mathcal{R} u(x, t)=\int_{C} f\left(x^{\prime}\right) \delta\left(\left\langle x, x^{\prime}\right\rangle-t\right) d \mu\left(x^{\prime}\right)
$$

is well-defined for $(x, t) \in\left(\mathbb{R}^{p+q} \backslash\{0\}\right) \times(\mathbb{R} \backslash\{0\})$, but we need here only the case where $x \in C$.

We rewrite the right-hand side of $(5.1 .4)$ for $u \in C_{0}^{\infty}(C)$ as follows:

$$
\begin{aligned}
\left(\mathcal{F}_{C} u\right)(x) & :=\int_{C} K\left(x, x^{\prime}\right) u\left(x^{\prime}\right) d \mu\left(x^{\prime}\right) \\
& =c_{p, q} \int_{\mathbb{R}^{p+q-2}} \Phi_{p, q}\left(\left\langle x, x^{\prime}\right\rangle\right) T u\left(x^{\prime}\right) d x^{\prime} \quad \text { by (5.1.1) } \\
& =c_{p, q} \int_{\mathbb{R}} \int_{\left\langle x, x^{\prime}\right\rangle=t} \Phi_{p, q}\left(\left\langle x, x^{\prime}\right\rangle\right) T u\left(x^{\prime}\right) d \omega\left(x^{\prime}\right) d t \\
& =c_{p, q} \int_{\mathbb{R}} \Phi_{p, q}(t) \mathcal{R} u(x, t) d t .
\end{aligned}
$$

Lemma 5.2.2. The right-hand side of $(5.2 .2)$ is well-defined for $u \in C_{0}^{\infty}(C)$.

Proof. It follows from Theorem 6.2.1 which we shall prove later and from the definition (5.1.3) of the distribution $\Phi_{p, q}(t)$ that $\Phi_{p, q}(t)$ has the following decomposition:

$$
\Phi_{p, q}(t)=\Phi_{p, q}^{\mathrm{reg}}(t)+\Phi_{p, q}^{\mathrm{sing}}(t),
$$

where $\Phi_{p, q}^{\mathrm{reg}}(t)$ and $\Phi_{p, q}^{\mathrm{sing}}(t)$ are distributions on $\mathbb{R}$ such that

1) $\Phi_{p, q}^{\mathrm{reg}}(t)|t|^{-\epsilon}$ is a locally integrable function on $\mathbb{R}$ for any sufficiently small $\epsilon \geq 0$, 
2)

$$
\Phi_{p, q}^{\text {sing }}(t)= \begin{cases}0 & \text { if } \min (p, q)=2 \\ -\sum_{k=1}^{\frac{p+q-6}{2}} \frac{(-1)^{k-1}}{2^{k}(m-k) !} \delta^{(k-1)}(t) & \text { if } p, q>2 \text { both even } \\ -\frac{1}{\pi} \sum_{k=1}^{\frac{p+q-6}{2}} \frac{(k-1) !}{2^{k}(m-k) !} t^{-k} & \text { if } p, q>2 \text { both odd }\end{cases}
$$

We note that $\Phi_{p, q}^{\text {sing }}(t) \neq 0$ only if $p, q>2$ and $p+q \geq 8$. Combining with Lemma 5.2.1, we see that the right-hand side of (5.2.2) is well-defined in all the cases.

Lemma 5.2 .2 defines a linear map

$$
\mathcal{F}_{C}: C_{0}^{\infty}(C) \rightarrow C^{\infty}(C),
$$

and defines $K\left(x, x^{\prime}\right)$ as a distribution on the direct product manifold $C \times C$. In Section 5.4, we shall see that the image $\mathcal{F}_{C}\left(C_{0}^{\infty}(C)\right)$ is contained in $L^{2}(C)$, and $\mathcal{F}_{C}$ extends to a unitary operator on $L^{2}(C)$, which in turn equals the unitary inversion operator $\pi\left(w_{0}\right)$.

Remark 5.2.3. The plane wave expansion gives a decomposition of the Euclidean Fourier transform $\mathcal{F}_{\mathbb{R}^{n}}$ on $L^{2}\left(\mathbb{R}^{n}\right)$ into the one-dimensional integral transform (Mellin transform) and the Radon transform, namely:

$$
\left(\mathcal{F}_{\mathbb{R}^{n}} u\right)(x)=c_{n}\langle\Psi,(R u)(x, \cdot)\rangle,
$$

where $c_{n}:=\left(\frac{1}{2 \pi}\right)^{\frac{n}{2}}$ and $\Psi(t):=e^{\sqrt{-1} t}$ (e.g. [25, Chapter I, §1.2]). In this sense, the formula (5.2.2) can be regarded as an analog of the plane wave expansion for the unitary operator $\pi\left(w_{0}\right)$ on $L^{2}(C)$.

\subsection{Spectra of $K^{\prime}$-invariant operators on $S^{p-2} \times S^{q-2}$}

The expansion into spherical harmonics

$$
L^{2}\left(S^{n-1}\right) \simeq \sum_{l=0}^{\infty} \mathcal{H}^{l}\left(\mathbb{R}^{n}\right)
$$

gives a multiplicity-free decomposition of $O(n)$ into its irreducible representations (see Appendix 7.5), and consequently, any $O(n)$-intertwining operator on $L^{2}\left(S^{n-1}\right)$ acts on $\mathcal{H}^{l}\left(\mathbb{R}^{n}\right)$ as a scalar multiplication owing to Schur's 
lemma. The scalar is given by the Funk-Hecke formula (see [1, §9.7], see also [46, Lemma 5.5.1]): for an integrable function $h$ on the interval $[-1,1]$ and for $\phi \in \mathcal{H}^{l}\left(\mathbb{R}^{n}\right)$,

$$
\int_{S^{n-1}} h\left(\left\langle\omega, \omega^{\prime}\right\rangle\right) \phi\left(\omega^{\prime}\right) d \omega^{\prime}=c_{l, n}(h) \phi(\omega)
$$

where the eigenvalue $c_{l, n}(h)$ is given by

$$
c_{l, n}(h)=\frac{2^{n-2} \pi^{\frac{n-2}{2}} l !}{\Gamma(n-2+l)} \int_{-1}^{1} h(x) \widetilde{C}_{l}^{\frac{n-2}{2}}(x)\left(1-x^{2}\right)^{\frac{n-3}{2}} d x .
$$

Here, $\widetilde{C}_{l}^{\mu}(x)$ stands for the normalized Gegenbauer polynomial (see Section 7.4).

Likewise, any $K^{\prime}$-intertwining operator on $L^{2}\left(S^{p-2} \times S^{q-2}\right)$ acts on the subspace $\mathcal{H}^{l}\left(\mathbb{R}^{p-1}\right) \otimes \mathcal{H}^{k}\left(\mathbb{R}^{q-1}\right)$ as a scalar multiplication for each $k, l \in \mathbb{N}$ (we recall $K^{\prime} \simeq O(p-1) \times O(q-1)$ ). In this section, we determine this scalar for specific intertwining (integral) operators. In particular, the scalar in Example 5.3.2 will be used in the proof of our main theorem (Theorem 5.1.1).

We begin with a general setup for a $K^{\prime}$-intertwining operator on $L^{2}\left(S^{p-2} \times\right.$ $\left.S^{q-2}\right)$. Let $h$ be an integrable function of two variables on $[-1,1] \times[-1,1]$. We consider the following integral transform:

$$
\begin{aligned}
B_{h}: C\left(S^{p-2} \times S^{q-2}\right) & \rightarrow C\left(S^{p-2} \times S^{q-2}\right), \\
\varphi(\omega, \eta) & \mapsto \int_{S^{p-2} \times S^{q-2}} h\left(\left\langle\omega, \omega^{\prime}\right\rangle,\left\langle\eta, \eta^{\prime}\right\rangle\right) \varphi\left(\omega^{\prime}, \eta^{\prime}\right) d \omega^{\prime} d \eta^{\prime} .
\end{aligned}
$$

Lemma 5.3.1. $B_{h}$ acts on each $K^{\prime}$-type $\mathcal{H}^{l}\left(\mathbb{R}^{p-1}\right) \otimes \mathcal{H}^{k}\left(\mathbb{R}^{q-1}\right)$ by a scalar multiplication of $\alpha_{l, k}(h) \in \mathbb{C}$. The spectrum $\alpha_{l, k}(h)$ is given by the following formulas.

1) If $\min (p, q)=2$, say $q=2$, then for $k=0,1$,

$$
\alpha_{l, k}(h)=\frac{2^{p-3} \pi^{\frac{p-3}{2}} l !}{\Gamma(p-3+l)} \int_{-1}^{1}\left(U_{k} h\right)(x) \widetilde{C}_{l}^{\frac{p-3}{2}}(x)\left(1-x^{2}\right)^{\frac{p-4}{2}} d x
$$

where we set

$$
\left(U_{k} h\right)(x):=h(x, 1)+(-1)^{k} h(x,-1) .
$$

For $k \geq 2, \alpha_{l, k}(h)=0$. 
2) If $p, q>2$, then

$$
\begin{aligned}
& \alpha_{l, k}(h)=\frac{2^{p+q-6} \pi^{\frac{p+q-6}{2}} l ! k !}{\Gamma(p-3+l) \Gamma(q-3+k)} \\
& \quad \times \int_{-1}^{1} \int_{-1}^{1} h(x, y) \widetilde{C}_{l}^{\frac{p-3}{2}}(x) \widetilde{C}_{k}^{\frac{q-3}{2}}(y)\left(1-x^{2}\right)^{\frac{p-4}{2}}\left(1-y^{2}\right)^{\frac{q-4}{2}} d x d y .
\end{aligned}
$$

Proof. 1) If $q=2$, then $S^{p-1} \times S^{q-1}=S^{p-1} \amalg S^{p-1}$ (disjoint union), and $\mathcal{H}^{k}\left(\mathbb{R}^{q-1}\right)=0$ if $k \geq 2$ (see Section 7.5 ). Then, the formula (5.3.3) is essentially the Funk-Hecke formula (5.3.1) for $S^{p-1}$.

2) Applying (5.3.1) to each factor, we get (5.3.5).

Let us give some examples of the explicit computation of spectra $\alpha_{l, k}(h)$.

Example 5.3.2 (Riesz potential). Consider the following Riesz potential for $\operatorname{Re} \lambda>-1$ :

$$
\begin{aligned}
h_{\lambda}^{ \pm}(x, y) & :=\frac{(x+y)_{ \pm}^{\lambda}}{\Gamma(\lambda+1)}, \\
& = \begin{cases}\frac{(x+y)^{\lambda}}{\Gamma(\lambda+1)} & \text { if } \epsilon(x+y)>0, \\
0 & \text { if } \epsilon(x+y) \leq 0,\end{cases}
\end{aligned}
$$

where $\epsilon= \pm 1$. Then, the spectrum $\alpha_{l, k}\left(h_{\lambda}^{ \pm}\right)$for the $K^{\prime}$-intertwining operator $B_{h_{\lambda}^{ \pm}}$amounts to

$$
\begin{aligned}
\alpha_{l, k}\left(h_{\lambda}^{ \pm}\right) & =\frac{2^{1-\lambda} \pi^{\frac{p+q-2}{2}}( \pm 1)^{l+k} \Gamma\left(\lambda+\frac{p+q-4}{2}\right)}{\Gamma\left(\frac{\lambda+p+q-4+l+k}{2}\right) \Gamma\left(\frac{\lambda+p-1+l-k}{2}\right) \Gamma\left(\frac{\lambda+q-1-l+k}{2}\right) \Gamma\left(\frac{\lambda-l-k+2}{2}\right)} \\
& =\frac{( \pm 1)^{l+k}}{\pi} \Gamma\left(\lambda+\frac{p+q-4}{2}\right) \sin \left(\frac{\lambda-l-k+2}{2} \pi\right) \sin \left(\frac{\lambda+q-1-l+k}{2} \pi\right) \gamma_{l, k}(\lambda),
\end{aligned}
$$

where we set

$$
\gamma_{l, k}(\lambda):=2^{1-\lambda} \pi^{\frac{p+q-4}{2}} \frac{\Gamma\left(\frac{l+k-\lambda}{2}\right) \Gamma\left(\frac{-q+3+l-k-\lambda}{2}\right)}{\Gamma\left(\frac{\lambda+p+q+l+k-4}{2}\right) \Gamma\left(\frac{\lambda+p-1+l-k}{2}\right)} .
$$

Proof of Example 5.3.2. Use (5.3.5). We postpone the actual computation of the integral (the first equation of $\alpha_{l, k}\left(h_{\lambda}^{ \pm}\right)$) until Appendix (see Lemma 7.9.1 with $\left.\mu=\frac{p-3}{2}, \nu=\frac{q-3}{2}\right)$. In the second equation of $\alpha_{l, k}\left(h_{\lambda}^{ \pm}\right)$, we have used the functional equation $\Gamma(z) \Gamma(1-z)=\frac{\pi}{\sin (z \pi)}$. 
follows:

We define a kernel function $h_{\lambda}(x, y) \equiv h_{\lambda}^{p, q}(x, y)$ with parameter $\lambda$ as $h_{\lambda}(x, y):=\frac{\Gamma(-\lambda)}{\Gamma\left(\lambda+\frac{p+q-4}{2}\right)} \times \begin{cases}(x+y)_{+}^{\lambda} & \text { if } p, q>2 \text { both even, } \\ \left(\frac{(x+y)_{+}^{\lambda}}{\tan \lambda \pi}+\frac{(x+y)_{-}^{\lambda}}{\sin \lambda \pi}\right) & \text { if } p, q>2 \text { both odd }\end{cases}$

Proposition 5.3.3. Let $\operatorname{Re} \lambda>-1$. For a kernel function $h_{\lambda}$ (see (5.3.8)), the spectrum $\alpha_{l, k}\left(h_{\lambda}\right)$ given in Lemma 5.3.1 amounts to

$$
\begin{aligned}
\alpha_{l, k}\left(h_{\lambda}\right) & =\frac{(-1)^{l+\left[\frac{q-3}{2}\right]} \pi^{\frac{p+q-4}{2}}}{2^{\lambda}} \frac{\Gamma\left(\frac{l+k-\lambda}{2}\right) \Gamma\left(\frac{-q+3+l-k-\lambda}{2}\right)}{\Gamma\left(\frac{\lambda+p+q+l+k-4}{2}\right) \Gamma\left(\frac{\lambda+p-1+l-k}{2}\right)} \\
& =\frac{(-1)^{k+\left[\frac{p-3}{2}\right]} \pi^{\frac{p+q-4}{2}}}{2^{\lambda}} \frac{\Gamma\left(\frac{l+k-\lambda}{2}\right) \Gamma\left(\frac{-p+3-l+k-\lambda}{2}\right)}{\Gamma\left(\frac{p+q-4+l+k+\lambda}{2}\right) \Gamma\left(\frac{q-1-l+k+\lambda}{2}\right)} .
\end{aligned}
$$

Proof. The second equation (5.3.10) follows from the identity (4.5.4) of gamma functions. Let us show the first equation (5.3.9). In terms of $h_{\lambda}^{ \pm}$ defined in (5.3.6), we rewrite $h_{\lambda}$ (see (5.3.8)) as

$$
h_{\lambda}=\frac{\pi}{\Gamma\left(\lambda+\frac{p+q-4}{2}\right) \sin (-\lambda \pi)} \times \begin{cases}h_{\lambda}^{+} & \text {if } p, q \text { both even } \\ \frac{h_{\lambda}^{+}}{\tan (\lambda \pi)}+\frac{h_{\lambda}^{-}}{\sin (\lambda \pi)} & \text { if } p, q \text { both odd } .\end{cases}
$$

Since $\alpha_{l, k}$ is linear, i.e., $\alpha_{l, k}(a h+b g)=a \alpha_{l, k}(h)+b \alpha_{l, k}(g), a, b \in \mathbb{C}$, by (5.3.11), we have

$$
\alpha_{l, k}\left(h_{\lambda}\right)=C_{l, k}(\lambda) \gamma_{l, k}(\lambda),
$$

where $\gamma_{l, k}(\lambda)$ is the meromorphic function given by (5.3.7), and

$C_{l, k}(\lambda):=\frac{\sin \frac{\lambda-l-k+2}{2} \pi \sin \frac{\lambda+q-1-l+k}{2} \pi}{\sin (-\lambda \pi)} \times \begin{cases}1 & \text { if } p, q \text { both even } \\ \frac{1}{\tan (\lambda \pi)}+\frac{(-1)^{l+k}}{\sin (\lambda \pi)} & \text { if } p, q \text { both odd }\end{cases}$

Hence, the proof of Proposition will be completed by the following claim:

\section{Claim 5.3.4.}

$$
C_{l, k}(\lambda)=\frac{(-1)^{l+\left[\frac{q-1}{2}\right]}}{2}
$$


Proof. Let us first consider the case where both $p$ and $q$ are even. Then, the two integers $-l-k+2$ and $q-1-l+k$ have different parities. Hence,

$$
C_{l, k}(\lambda)=(-1)^{l+\frac{q-2}{2}} \frac{\sin \frac{\lambda}{2} \pi \cos \frac{\lambda}{2} \pi}{\sin \lambda \pi}=\frac{(-1)^{l+\frac{q-2}{2}}}{2} .
$$

Next, suppose both $p$ and $q$ are odd. Then,

$$
\begin{aligned}
& \frac{1}{\tan (\lambda \pi)}+\frac{(-1)^{l+k}}{\sin (\lambda \pi)}=\left\{\begin{array}{l}
\frac{1}{\tan \frac{\lambda}{2} \pi} \\
-\tan \frac{\lambda}{2} \pi
\end{array}\right. \\
& \frac{\sin \frac{\lambda-l-k+2}{2} \pi \sin \frac{\lambda+q-1-l+k}{2} \pi}{\sin (-\lambda) \pi}=\left\{\begin{array}{l}
(-1)^{-l+\frac{q-1}{2}} \frac{\sin ^{2} \frac{\lambda}{2} \pi}{\sin \lambda \pi}=\frac{(-1)^{-l+\frac{q-1}{2}}}{2} \tan \frac{\lambda}{2} \pi \\
(-1)^{-l+\frac{q+1}{2}} \frac{\cos ^{2} \frac{\lambda}{2} \pi}{\sin \lambda \pi}=\frac{(-1)^{-l+\frac{q+1}{2}}}{2} \frac{1}{\tan \frac{\lambda}{2} \pi}
\end{array}\right.
\end{aligned}
$$

according as $l+k$ is even (upper row) and odd (lower row). Thus we have

$$
C_{l, k}(\lambda)=\frac{(-1)^{\frac{q-1}{2}-l}}{2}
$$

in either case. Hence, Claim 5.3 .4 is verified.

Let $T$ be the triangular domain in $\mathbb{R}^{2}$ given by

$$
T:=\left\{(x, y) \in \mathbb{R}^{2}: x<1, y<1,0<x+y\right\},
$$

and define a function $g_{\lambda}(x, y)$ with parameter $\lambda \in \mathbb{C}$ by

$$
g_{\lambda}(x, y):= \begin{cases}(x+y)^{\lambda}\left(1-x^{2}\right)^{\frac{p-4}{2}}\left(1-y^{2}\right)^{\frac{q-4}{2}} & (x, y) \in T, \\ 0 & (x, y) \notin T .\end{cases}
$$

Lemma 5.3.5. 1) For $\operatorname{Re} \lambda>-1, g_{\lambda}$ is a distribution of compact support, and with holomorphic parameter $\lambda$. That is, $\left\langle g_{\lambda}, \varphi\right\rangle$ is holomorphic in $\{\lambda \in$ $\mathbb{C}: \operatorname{Re} \lambda>-1\}$ for any $\varphi \in C^{\infty}\left(\mathbb{R}^{2}\right)$.

2) $g_{\lambda}$ extends as a distribution with a meromorphic parameter $\lambda \in \mathbb{C}$. That is, $\left\langle g_{\lambda}, \varphi\right\rangle$ is a meromorphic function with respect to $\lambda \in \mathbb{C}$ for any $\varphi \in C^{\infty}\left(\mathbb{R}^{2}\right)$.

Proof. The first statement is clear because $g_{\lambda} \in L^{1}\left(\mathbb{R}^{2}\right)$ if $\operatorname{Re} \lambda>-1$. For the second statement, we rewrite $g_{\lambda}$ as

$$
g_{\lambda}(x, y)=(x+y)_{+}^{\lambda}(1-x)_{+}^{\frac{p-4}{2}}(1+x)_{+}^{\frac{p-4}{2}}(1-y)_{+}^{\frac{q-4}{2}}(1+y)_{+}^{\frac{q-4}{2}} .
$$

Then, Lemma follows from Bernstein's theorem [5]. 


\subsection{Proof of Theorem 5.1.1}

We recall from (5.1.4) that $\mathcal{F}_{C}$ is the linear map defined by

$$
u(x) \mapsto \int_{C} K\left(x, x^{\prime}\right) u\left(x^{\prime}\right) d \mu\left(x^{\prime}\right) .
$$

Since $\mathcal{F}_{C}$ commutes with the $K^{\prime}$-action $\left(K^{\prime} \simeq O(p-1) \times O(q-1)\right)$, $\mathcal{F}_{C}$ preserves each $K^{\prime}$-isotypic component of $L^{2}(C)$ given in the decomposition (see (4.1.1)):

$$
L^{2}(C) \simeq \sum_{l, k=0}^{\infty} L^{2}\left(\mathbb{R}_{+}, \frac{1}{2} r^{p+q-5} d r\right) \otimes \mathcal{H}^{l}\left(\mathbb{R}^{p-1}\right) \otimes \mathcal{H}^{k}\left(\mathbb{R}^{q-1}\right) .
$$

On the other hand, we have seen in Theorem 4.1.1 that $\pi\left(w_{0}\right)$ also preserves each $K^{\prime}$-isotypic component, and accordingly has a decomposition:

$$
\pi\left(w_{0}\right)=\sum_{l, k=0}^{\infty} T_{l, k} \otimes \mathrm{id} \otimes \mathrm{id}
$$

where $T_{l, k}$ is a unitary operator on $L^{2}\left(\mathbb{R}_{+}, \frac{1}{2} r^{p+q-5} d r\right)$ whose kernel $K_{l, k}(t)$ is explicitly given in (4.1.2).

We shall show the equality $\mathcal{F}_{C}=\pi\left(w_{0}\right)$ by restricting to each $(l, k)$ component, namely,

Lemma 5.4.1. For each $l, k \in \mathbb{N}$, we have

$$
\left.\mathcal{F}_{C}\right|_{L^{2}\left(\mathbb{R}_{+}, r^{p+q-5} d r\right) \otimes \mathcal{H}^{l}\left(\mathbb{R}^{p-1}\right) \otimes \mathcal{H}^{k}\left(\mathbb{R}^{q-1}\right)}=T_{l, k} \otimes \mathrm{id} \otimes \mathrm{id} .
$$

Instead of proving Lemma 5.4.1, we shall prove Lemma 5.4.2 on the spectra $\alpha_{l, k}$ and the kernel functions $K_{l, k}$, which turns out to be equivalent to Lemma 5.4.1. For that purpose, we set

$$
h_{r, r^{\prime}}(x, y):=c_{p, q} \Phi_{p, q}\left(r r^{\prime}(x+y)\right) \text {, }
$$

where $c_{p, q}$ and $\Phi_{p, q}$ are defined in (5.1.2) and (5.1.3). Then, by the definition (5.1.1) of $K\left(x, x^{\prime}\right)$, we have

$$
\begin{aligned}
K\left(\left(\begin{array}{c}
r \omega \\
r \eta
\end{array}\right),\left(\begin{array}{l}
r^{\prime} \omega^{\prime} \\
r^{\prime} \eta^{\prime}
\end{array}\right)\right) & =c_{p, q} \Phi_{p, q}\left(r r^{\prime}\left(\left\langle w, w^{\prime}\right\rangle+\left\langle\eta, \eta^{\prime}\right\rangle\right)\right) \\
& =h_{r, r^{\prime}}\left(\left\langle\omega, \omega^{\prime}\right\rangle,\left\langle\eta, \eta^{\prime}\right\rangle\right) .
\end{aligned}
$$


Suppose $f(r) u(\omega, \eta) \in L^{2}\left(\mathbb{R}_{+}, r^{p+q-5} d r\right) \otimes \mathcal{H}^{l}\left(\mathbb{R}^{p-1}\right) \otimes \mathcal{H}^{k}\left(\mathbb{R}^{q-1}\right)$.

$$
\begin{aligned}
\mathcal{F}_{C}(f u)(r \omega, r \eta) \\
=\int_{C} K\left(\left(\begin{array}{c}
r \omega \\
r \eta
\end{array}\right), x^{\prime}\right)(f u)\left(x^{\prime}\right) d \mu\left(x^{\prime}\right) \\
=\frac{1}{2} \int_{0}^{\infty} \int_{S^{p-2}} \int_{S^{q-2}} h_{r, r^{\prime}}\left(\left\langle\omega, \omega^{\prime}\right\rangle,\left\langle\eta, \eta^{\prime}\right\rangle\right) f\left(r^{\prime}\right) u\left(\omega^{\prime}, \eta^{\prime}\right) r^{\prime p+q-5} d r^{\prime} d \omega^{\prime} d \eta^{\prime} \\
=\frac{1}{2} \int_{0}^{\infty}\left(B_{h_{r, r^{\prime}}} u\right) f\left(r^{\prime}\right) r^{p+q-5} d r^{\prime}
\end{aligned}
$$

Since $B_{h_{r, r^{\prime}}} u=\alpha_{l, k}\left(h_{r, r^{\prime}}\right) u$ by Lemma [5.3.1, we have

$$
\mathcal{F}_{C}(f u)(r \omega, r \eta)=\frac{1}{2} \int_{0}^{\infty} \alpha_{l, k}\left(h_{r, r^{\prime}}\right) f\left(r^{\prime}\right) r^{\prime p+q-5} d r^{\prime} u(\omega, \eta) .
$$

On the other hand, it follows from Theorem 4.1.1 (2) that

$$
\begin{aligned}
& \left(\left(T_{l, k} \otimes \mathrm{id} \otimes \mathrm{id}\right)(f u)\right)(r \omega, r \eta) \\
& =\left(T_{l, k} f\right)(r) u(\omega, \eta) \\
& =\frac{1}{2} \int_{0}^{\infty} K_{l, k}\left(r r^{\prime}\right) f\left(r^{\prime}\right) r^{\prime p+q-5} d r^{\prime} u(\omega, \eta) .
\end{aligned}
$$

Since $L^{2}\left(\mathbb{R}_{+}, r^{p+q-5} d r\right) \otimes \mathcal{H}^{l}\left(\mathbb{R}^{p-1}\right) \otimes \mathcal{H}^{k}\left(\mathbb{R}^{q-1}\right)$ is spanned by a linear combination of the function of the form $f(r) u(\omega, \eta)$, Lemma 5.4 .1 is equivalent to the following formula between kernel functions:

Lemma 5.4.2. For each $l, k \in \mathbb{N}$, we have

$$
\alpha_{l, k}\left(h_{r, r^{\prime}}\right)=K_{l, k}\left(r r^{\prime}\right) .
$$

The proof of Lemma 5.4.2 will be given in the following two Sections, which will then complete the proof of Theorem 5.1.1.

\subsection{Proof of Lemma 5.4.2 (Hermitian case $q=2$ )}

This section gives a proof of Lemma 5.4 .2 in the case $\min (p, q)=2$. Without loss of generality, we may and do assume $q=2$. By the definition (5.1.3) of $\Phi_{p, 2}(t)$, the definition (5.4.2) of $h_{r, r^{\prime}}$ amounts to:

$$
h_{r, r^{\prime}}(x, y)=c_{p, 2} \Phi_{\frac{p-4}{2}}^{+}\left(r r^{\prime}(x+y)\right) .
$$


Since $\Phi_{\frac{p-4}{2}}^{+}(t)$ is a locally integrable function supported on $t \geq 0$ (see Theorem 6.2.1), we have from the definition (5.3.4) of the operator $U_{k}(k=$ $0,1)$ : for $-1 \leq x \leq 1$,

$$
\begin{aligned}
\left(U_{k} h_{r, r^{\prime}}\right)(x) & =c_{p, 2} \Phi_{\frac{p-4}{2}}^{+}\left(r r^{\prime}(x+1)\right)+(-1)^{k} c_{p, 2} \Phi_{\frac{p-4}{2}}^{+}\left(r r^{\prime}(x-1)\right) \\
& =c_{p, 2} \Phi_{\frac{p-4}{2}}^{+}\left(r r^{\prime}(x+1)\right) .
\end{aligned}
$$

Then, by (1.3.1), we have:

$$
=\frac{2^{-\frac{p-8}{4}}(-1)^{\frac{(p-1)(p+2)}{2}}}{\pi^{\frac{p-2}{2}}}\left(r r^{\prime}\right)^{-\frac{p-4}{4}}(x+1)^{-\frac{p-4}{4}} J_{\frac{p-4}{2}}\left(2 \sqrt{2 r r^{\prime}(x+1)}\right) .
$$

Applying the Funk-Hecke formula (5.3.3), the spectrum $\alpha_{l, k}\left(h_{r, r^{\prime}}\right)$ amounts to

$$
\begin{aligned}
\alpha_{l, k}\left(h_{r, r^{\prime}}\right)= & \frac{2^{\frac{3 p-4}{4}}(-1)^{\frac{(p-1)(p+2)}{2}} l !}{\sqrt{\pi} \Gamma(p-3+l)}\left(r r^{\prime}\right)^{-\frac{p-4}{4}} \times \\
& \int_{-1}^{1} J_{\frac{p-4}{2}}\left(2 \sqrt{2 r r^{\prime}(x+1)}\right) \widetilde{C}_{l}^{\frac{p-3}{2}}(x)(1+x)^{\frac{p-4}{4}}(1-x)^{\frac{p-4}{2}} d x \\
= & 4(-1)^{\frac{(p-1)(p+2)}{2}+l}\left(r r^{\prime}\right)^{-\frac{p-3}{2}} J_{p-3+2 l}\left(4 \sqrt{r r^{\prime}}\right) \\
= & (-1)^{\frac{p^{2}}{2}} K_{l, k}\left(r r^{\prime}\right) .
\end{aligned}
$$

Here, the second equality follows from the integral formula (7.4.10) of the Gegenbauer polynomials in Appendix with $\alpha=2 \sqrt{2 r r^{\prime}}$ and $\nu=\frac{p-4}{2}$, and the last equality follows from (4.1.4). Since $p$ is even in the case $q=2$, the right-hand side is equal to $K_{l, k}\left(r r^{\prime}\right)$. Hence, Lemma 5.4.2 is proved for $q=2$.

\subsection{Proof of Lemma $\mathbf{5 . 4 . 2}(p, q>2)$}

This section gives a proof of Lemma $\lcm{5.4 .2}$ in the general case $p, q>2$.

First, we give an integral formula of Mellin-Barnes type for $h_{r, r^{\prime}}(x, y)$ (see (5.4.2) ) by means of $h_{\lambda}(x, y)$ (see (5.3.8) for definition): Suppose $p, q>$ 2 .

Claim 5.6.1. Let $\gamma>-1$ and $L$ be a contour that starts at $\gamma-\sqrt{-1} \infty$ and ends at $\gamma+\sqrt{-1} \infty$. We assume that $L$ passes the real axis in the interval $\left(-\frac{p+q-4}{2},-\frac{p+q-6}{2}\right)$. Then, we have

$$
h_{r, r^{\prime}}(x, y)=\frac{c_{p, q}}{2 \pi \sqrt{-1}} \int_{L}\left(2 r r^{\prime}\right)^{\lambda} h_{\lambda}(x, y) d \lambda .
$$


Proof. By the definition (5.3.8) of $h_{\lambda}(x, y) \equiv h_{\lambda}^{p, q}(x, y)$ and the integral formulas $(6.2 .2)$ and $(6.2 .4)$ of $\Psi_{\frac{p+q-6}{2}}^{+}(t)$ and $\frac{\Psi_{\frac{p+q-6}{2}}}{2}(t)$ respectively, we have

$$
\frac{1}{2 \pi \sqrt{-1}} \int_{L} s^{\lambda} h_{\lambda}(x, y) d \lambda= \begin{cases}\Psi_{\frac{p+q-6}{2}\left(\frac{s(x+y)}{2}\right)}^{+} & p, q \text { even, } \\ \Psi_{\frac{p+q-6}{2}}\left(\frac{s(x+y)}{2}\right) & p, q \text { odd }\end{cases}
$$

for $s>0$. In either case, it follows from the definition(5.1.3) of $\Phi_{p, q}(t)$ that

$$
\frac{1}{2 \pi \sqrt{-1}} \int_{L} s^{\lambda} h_{\lambda}(x, y) d \lambda=\Phi_{p, q}\left(\frac{s(x+y)}{2}\right) .
$$

Hence, we get Claim 5.6.1 by the definition (5.4.2) of $h_{r, r^{\prime}}(x, y)$.

By the linearity of $\alpha_{l, k}$, we have

$$
\begin{aligned}
\alpha_{l, k}\left(h_{r, r^{\prime}}\right) & =\frac{c_{p, q}}{2 \pi \sqrt{-1}} \int_{L} \alpha_{l, k}\left(h_{\lambda}\right)\left(2 r r^{\prime}\right)^{\lambda} d \lambda \\
& =\frac{(-1)^{l+\frac{p-q}{2}}}{\pi \sqrt{-1}} \int_{L} \frac{\Gamma\left(\frac{l+k-\lambda}{2}\right) \Gamma\left(\frac{-q+3+l-k-\lambda}{2}\right)}{\Gamma\left(\frac{\lambda+p+q+l+k-4}{2}\right) \Gamma\left(\frac{\lambda+p-1+l-k}{2}\right)}\left(r r^{\prime}\right)^{\lambda} d \lambda \\
& =K_{l, k}\left(r r^{\prime}\right) .
\end{aligned}
$$

Here, in the second equality, we applied Proposition 5.3.3 and then used the equality $(-1)^{l+\left[\frac{q-1}{2}\right]}(-1)^{\frac{(p-1)(p+2)}{2}}=(-1)^{l+\frac{p-q}{2}}$, which follows from the congruence equality:

$$
\frac{(p-1)(p+2)}{2}+\left[\frac{q-1}{2}\right] \equiv \frac{p-q}{2} \bmod 2
$$

under the assumption that $p+q$ is even. The last equality follows from Lemma 4.5.2. Hence, we have proved Lemma 5.4.2 in the general case $p, q>2$.

Proof of Theorem 5.1.1. Now, Lemma 5.4.2 is proved in all the cases. Hence, the proof of Theorem 5.1 .1 is completed. 


\section{Chapter 6}

\section{Bessel distributions}

We have seen in the previous chapter (see Theorem 5.1.1) that the unitary inversion operator $\pi\left(w_{0}\right): L^{2}(C) \rightarrow L^{2}(C)$ is given by the distribution kernel $K\left(x, x^{\prime}\right)$ which is the composition of the restriction of the bilinear map

$$
C \times C \rightarrow \mathbb{R}, \quad\left(x, x^{\prime}\right) \mapsto\left\langle x, x^{\prime}\right\rangle
$$

and Bessel distributions (see (1.3.1) $-(1.3 .3)$ ) of one variable. In this chapter, we analyze the distribution kernel from three viewpoints: integral formulas, power series expansion (including distributions such as $\delta^{(k)}(x)$ and $x^{-k}$ ), and differential equations.

Section 6.3 gives a heuristic account on why $K\left(x, x^{\prime}\right)$ is essentially of one variable, and why the Bessel distribution arises in $K\left(x, x^{\prime}\right)$. The results of Section 6.3 is not used for other sections.

\subsection{Meijer's G-distributions}

In this section, we give a definition of Meijer's G-distributions which have the following two properties:

1) They are distributions on $\mathbb{R}$.

2) The restrictions to the positive half line $\{x>0\}$ are (usual) Meijer's $G$-functions (see Appendix 7.6).

The main result of this section is Proposition 6.1.2

Let $m, n, p$ and $q$ be integers with $0 \leq m \leq q$ and $0 \leq n \leq p$. Suppose moreover that the complex numbers $a_{1}, \ldots, a_{p}$ and $b_{1}, \ldots, b_{q}$ fulfill the condition

$$
a_{j}-b_{k} \neq 1,2,3, \ldots \quad(j=1, \ldots, n ; k=1, \ldots, m) .
$$


This means that no pole of the gamma function $\Gamma\left(b_{j}-\lambda\right)(j=1, \ldots, m)$ coincides with any pole of $\Gamma\left(1-a_{k}+\lambda\right)(k=1, \ldots, n)$. We set

$$
\begin{aligned}
c^{*} & :=m+n-\frac{p+q}{2}, \\
\mu & :=\sum_{j=1}^{q} b_{j}-\sum_{j=1}^{p} a_{j}+\frac{p-q}{2}+1 .
\end{aligned}
$$

Throughout this chapter, we assume $c^{*} \geq 0$. If $c^{*}=0$ then we also assume

$$
p-q<0 \quad \text { or } \quad p-q>\operatorname{Re} \mu .
$$

It is easy to see that the condition (6.1.3) allows us to find $\gamma \in \mathbb{R}$ such that

$$
\gamma>-1 \text { and }(q-p) \gamma>\operatorname{Re} \mu
$$

Remark 6.1.1. The conditions (6.1.3) and $\gamma>-1$ will not be used when we define (usual) Meijer's G-function as an analytic function in $x>0$ (see (7.6.2) ). They will be used in showing that Meijer's G-distribution $G\left(x_{+}\right)_{L}$ given by the Mellin-Barnes type integral (6.1.8) is a locally integrable function on $\mathbb{R}$ if we take an appropriate contour L (see Proposition 6.1.2 (3)).

We now take a contour $L$ which starts at the point $\gamma-\sqrt{-1} \infty$ and finishes at $\gamma+\sqrt{-1} \infty$. Later, we shall impose the following conditions on $L$ :

$L$ does not go through any negative integer.

$L$ leaves $b_{j}(1 \leq j \leq m)$ to the right, and $a_{j}-1(1 \leq j \leq n)$

to the left.

We note that the condition (6.1.6) implies:

$L$ does not go through any point in

$$
\left\{b_{j}+k: 1 \leq j \leq m, k \in \mathbb{N}\right\} \cup\left\{a_{j}-1-k: 1 \leq j \leq n, k \in \mathbb{N}\right\} .
$$

With these parameters, we define a meromorphic function of $\lambda$ by

$$
\Gamma_{p, q}^{m, n}\left(\lambda \mid \begin{array}{c}
a_{1}, \ldots, a_{p} \\
b_{1}, \ldots, b_{q}
\end{array}\right):=\frac{\prod_{j=1}^{m} \Gamma\left(b_{j}-\lambda\right) \prod_{j=1}^{n} \Gamma\left(1-a_{j}+\lambda\right)}{\prod_{j=m+1}^{q} \Gamma\left(1-b_{j}+\lambda\right) \prod_{j=n+1}^{p} \Gamma\left(a_{j}-\lambda\right)} .
$$


For $\operatorname{Re} \lambda>-1$, we set

$$
x_{+}^{\lambda}:=\left\{\begin{array}{ll}
x^{\lambda} & (x>0) \\
0 & (x \leq 0),
\end{array} \quad x_{-}^{\lambda}:= \begin{cases}0 & (x \geq 0) \\
|x|^{\lambda} & (x<0) .\end{cases}\right.
$$

Then, $x_{+}^{\lambda}$ and $x_{-}^{\lambda}$ are locally integrable functions of the variable $x$ in $\mathbb{R}$, and extend to distributions with meromorphic parameter $\lambda$ in the entire complex plane (see Appendix 7.1).

Proposition 6.1.2. Let $L$ be a contour satisfying $(6.1 .5)$ and $(6.1 .6)^{\prime}$.

1) The Mellin-Barnes type integral:

$$
\begin{aligned}
G\left(x_{+}\right)_{L} & \equiv G_{p, q}^{m, n}\left(x_{+} \mid \begin{array}{c}
a_{1}, \ldots, a_{p} \\
b_{1}, \ldots, b_{q}
\end{array}\right)_{L} \\
& :=\frac{1}{2 \pi \sqrt{-1}} \int_{L} \Gamma_{p, q}^{m, n}\left(\lambda \mid \begin{array}{c}
a_{1}, \ldots, a_{p} \\
b_{1}, \ldots, b_{q}
\end{array}\right) x_{+}^{\lambda} d \lambda
\end{aligned}
$$

is well-defined as a distribution on $\mathbb{R}$.

Its support is given by

$$
\operatorname{supp} G\left(x_{+}\right)_{L}=\{x \in \mathbb{R}: x \geq 0\} .
$$

2) If the contour $L$ satisfies (6.1.6), then the restriction of $G\left(x_{+}\right)_{L}$ to the positive half line $\{x \in \mathbb{R}: x>0\}$ is a real analytic function, which coincides with the (usual) G-function $G_{p, q}^{m, n}\left(x \mid \begin{array}{c}a_{1}, \ldots, a_{p} \\ b_{1}, \ldots, b_{q}\end{array}\right)$ (see (7.6.2) for definition).

3) If the contour $L$ is contained in the half plane $\{\lambda \in \mathbb{C}: \operatorname{Re} \lambda>-1\}$, then $G\left(x_{+}\right)_{L}$ is a locally integrable function on $\mathbb{R}$. More precisely, there exists $\epsilon_{0}>0$ such that $G\left(x_{+}\right)_{L} x_{+}^{-\epsilon}$ is locally integrable for any $\epsilon$ with $0 \leq$ $\epsilon<\epsilon_{0}$.

Likewise, we can define the distribution

$$
\begin{aligned}
G\left(x_{-}\right)_{L} & \equiv G_{p, q}^{m, n}\left(x_{-} \mid \begin{array}{c}
a_{1}, \ldots, a_{p} \\
b_{1}, \ldots, b_{q}
\end{array}\right)_{L} \\
& :=\frac{1}{2 \pi \sqrt{-1}} \int_{L} \Gamma_{p, q}^{m, n}\left(\lambda \mid \begin{array}{c}
a_{1}, \ldots, a_{p} \\
b_{1}, \ldots, b_{q}
\end{array}\right) x_{-}^{\lambda} d \lambda
\end{aligned}
$$

by using the same contour $L$, and the support of $G\left(x_{-}\right)_{L}$ is equal to the negative half line $\{x \in \mathbb{R}: x \leq 0\}$. 
Remark 6.1.3. The distribution $G\left(x_{ \pm}\right)_{L}$ depends on the choice of the contour $L$ even when we assume $L$ satisfies the conditions (6.1.5) and (6.1.6). In fact, if $L$ and $L^{\prime}$ are contours satisfying (6.1.5) and (6.1.6), then $G\left(x_{ \pm}\right)_{L}$ may differ from $G\left(x_{ \pm}\right)_{L^{\prime}}$ by a distribution supported at 0 , namely, a finite sum of Dirac's delta function and its derivatives. This is because the distribution $x_{ \pm}^{\lambda}$ has simple poles at $\lambda=-1,-2, \ldots$, and consequently, its residues (see (7.1.1) and (7.1.2) ) may appear when we move the contour $L$ across negative integers. In order to define the $G$-distribution in a unique fashion, we need to impose an additional constraint on the contour $L$. We shall work with concrete examples for this in Section 6.2 where we use Cauchy's integral formula for distributions with meromorphic parameter.

In order to prove Proposition 6.1.2, we need an asymptotic estimate of the $\Gamma$-factors in the integrand of $(6.1 .8)$ as follows:

Lemma 6.1.4. For any $\epsilon>0$, there exists a constant $C>0$ such that

$$
\left|\Gamma_{p, q}^{m, n}\left(\lambda \mid \begin{array}{c}
a_{1}, \ldots, a_{p} \\
b_{1}, \ldots, b_{q}
\end{array}\right)\right| \leq C e^{-\pi c^{*}|\operatorname{Im} \lambda|}|\operatorname{Im} \lambda|^{\operatorname{Re} \mu+(p-q) \gamma-1+\epsilon}
$$

for any $\lambda \in L$ such that $|\operatorname{Im} \lambda|$ is sufficiently large. Here, $c^{*}$ and $\mu$ are defined as in (6.1.1) and (6.1.2), and $\gamma=\lim _{\substack{\lambda \in L \\|\operatorname{Im} \lambda| \rightarrow \infty}} \operatorname{Re} \lambda$.

Proof. Fix $a \in \mathbb{C}$. By Stirling's asymptotic formula (4.5.8) of the gamma function, we have

$$
\begin{aligned}
|\Gamma(a-\lambda)| & =C_{a}|\operatorname{Im} \lambda|^{\operatorname{Re} a-\operatorname{Re} \lambda-\frac{1}{2}} e^{-\frac{\pi}{2}|\operatorname{Im} \lambda|}\left(1+O\left(|\operatorname{Im} \lambda|^{-1}\right)\right), \\
|\Gamma(1-a+\lambda)| & =C_{a}|\operatorname{Im} \lambda|^{-\operatorname{Re} a+\operatorname{Re} \lambda+\frac{1}{2}} e^{-\frac{\pi}{2}|\operatorname{Im} \lambda|}\left(1+O\left(|\operatorname{Im} \lambda|^{-1}\right)\right),
\end{aligned}
$$

as $|\operatorname{Im} \lambda|$ tends to infinity with $\operatorname{Re} \lambda$ bounded. Here, the constant $C_{a}$ is given by

$$
C_{a}=\sqrt{2 \pi} e^{-\frac{\pi}{2} \operatorname{sgn}(\operatorname{Im} \lambda)|\operatorname{Im} a|} .
$$

By the definition (6.1.7) of $\Gamma_{p, q}^{m, n}\left(\lambda \mid \begin{array}{c}a_{1}, \ldots, a_{p} \\ b_{1}, \ldots, b_{q}\end{array}\right)$, we now get the following asymptotic behavior:

$$
\left|\Gamma_{p, q}^{m, n}\left(\lambda \mid \begin{array}{c}
a_{1}, \ldots, a_{p} \\
b_{1}, \ldots, b_{q}
\end{array}\right)\right|=C^{\prime}|\operatorname{Im} \lambda|^{s} e^{-\frac{\pi}{2} t|\operatorname{Im} \lambda|}\left(1+O\left(|\operatorname{Im} \lambda|^{-1}\right)\right),
$$


as $|\operatorname{Im} \lambda|$ tends to infinity, where $C^{\prime}$ is a constant depending on $\operatorname{Im} a_{j}$ and $\operatorname{Im} b_{j}$, and

$$
\begin{aligned}
s & =\sum_{j=1}^{m} \operatorname{Re}\left(b_{j}-\lambda-\frac{1}{2}\right)+\sum_{j=1}^{n} \operatorname{Re}\left(-a_{j}+\lambda+\frac{1}{2}\right) \\
& -\sum_{j=m+1}^{q} \operatorname{Re}\left(\frac{1}{2}-b_{j}+\lambda\right)-\sum_{j=n+1}^{p} \operatorname{Re}\left(a_{j}-\lambda-\frac{1}{2}\right) \\
& =\operatorname{Re} \mu+(p-q) \operatorname{Re} \lambda-1, \\
t & =m+n-(q-m)-(p-n) \\
& =2 c^{*} .
\end{aligned}
$$

As $\operatorname{Re} \lambda$ converges to $\gamma$ when $\lambda \in L$ goes to infinity, we get Lemma 6.1.4.

We are ready to give a proof of Proposition 6.1.2.

Proof of Proposition 6.1.2. 3) We begin with the proof of the third statement. Suppose $L$ is contained in the half plane $\{\lambda \in \mathbb{C}: \operatorname{Re} \lambda>-1\}$. We need to show the integral (6.1.8) makes sense and gives rise to a locally integrable function of $x$. For the convergence of the integral, we shall use Lemma 6.1 .4 for the estimate as a function of $\lambda$. The non-trivial part is a uniform estimate in the neighborhood of $x=0$. Let us consider the interval $0<x \leq 1$.

Since the contour $L$ has the property:

$$
\gamma=\lim _{\substack{\lambda \in L \\|\operatorname{Im} \lambda| \rightarrow \infty}} \operatorname{Re} \lambda>-1
$$

the assumption $L \subset\{\lambda \in \mathbb{C}: \operatorname{Re} \lambda>-1\}$ implies $\delta>-1$, where we set

$$
\delta:=\inf _{\lambda \in L} \operatorname{Re} \lambda
$$

Hence, we get

$$
\left|x_{+}^{\lambda}\right| \leq x^{\delta} \quad \text { for } 0<x \leq 1 .
$$

On the other hand, it follows from Lemma 6.1.4 that

$$
\left|\Gamma_{p, q}^{m, n}\left(\lambda \mid \begin{array}{c}
a_{1}, \ldots, a_{p} \\
b_{1}, \ldots, b_{q}
\end{array}\right)\right| \leq \begin{cases}C e^{-\pi c^{*}|\operatorname{Im} \lambda|} & \text { if } c^{*}>0 \\
C|\operatorname{Im} \lambda|^{-1+\epsilon} & \text { if } c^{*}=0\end{cases}
$$


when $|\operatorname{Im} \lambda|$ is sufficiently large. Here, we used the inequality $\operatorname{Re} \mu+(p-$ $q) \gamma<0$ (see (6.1.4)) in the second case. Hence, $\Gamma_{p, q}^{m, n}\left(\lambda \mid \begin{array}{c}a_{1}, \ldots, a_{p} \\ b_{1}, \ldots, b_{q}\end{array}\right)$ is absolutely integrable on $L$ in either case. Therefore, the integration (6.1.8) converges, giving rise to a function of $x$ which is bounded by a scalar multiple of $x^{\delta}$ on the interval $0<x \leq 1$, whence a locally integrable function of $x$. Thus, $G\left(x_{+}\right)_{L}$ is locally integrable. Similarly, if we set

$$
\epsilon_{0}:=1+\delta \quad(>0)
$$

then for any $0 \leq \epsilon<\epsilon_{0}, x^{-\epsilon+\delta}$ is locally integrable, and consequently $G\left(x_{+}\right)_{L} x_{+}^{-\epsilon}$ is locally integrable. Hence, the third statement of Proposition is proved.

1) We divide the integral (6.1.8) into the sum of the following two integrals

$$
\int_{L}=\int_{L^{\prime}}+\int_{C}
$$

where $L^{\prime}$ is a contour contained in the right half plane $\{\lambda \in \mathbb{C}: \operatorname{Re} \lambda>-1\}$, and $C$ is the closed oriented curve given by $L-L^{\prime}$ (see Figure 6.1.1).

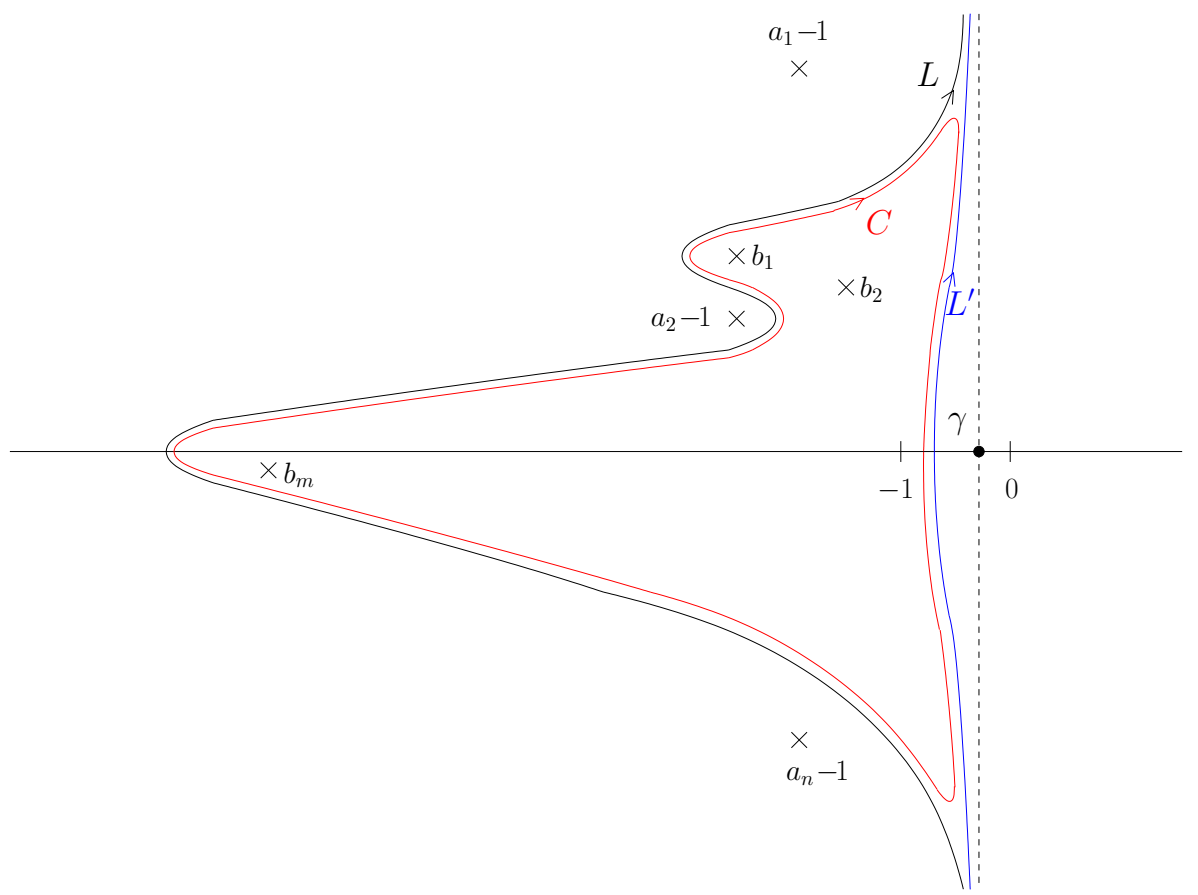

Figure 6.1.1 
Then, we have already seen that the second term gives a locally integrable function of $x$ (the third statement of this proposition). On the other hand, the third term is well-defined as a distribution because $C$ is compact and the integrand is a distribution of $x$ that depends continuously on $\lambda$ as far as $\lambda$ lies in $C$. Hence, the first statement is also proved.

2) This statement is well-known. See Appendix 7.6 for details.

\subsection{Integral expression of Bessel distributions}

In this section, we apply general results on Meijer's $G$-distributions developed in Section 6.1 to special cases, and obtain the Mellin-Barnes type integral expression for the distribution kernel of the unitary inversion operator $\pi\left(w_{0}\right)$.

Let $m$ be a non-negative integer. We take a contour $L$ such that

1) $L$ starts at $\gamma-\sqrt{-1} \infty$, passes the real axis at some point $s$, and ends at $\gamma+\sqrt{-1} \infty$.

2) $-1<\gamma$ and $-m-1<s<-m$.

Likewise, we take a contour $L_{0}$ (with analogous notation) such that

3) $-1<\gamma_{0}$ and $-1<s_{0}<0$.

For later purpose, we may and do take $\gamma=\gamma_{0}$. See Figure 6.2.1.

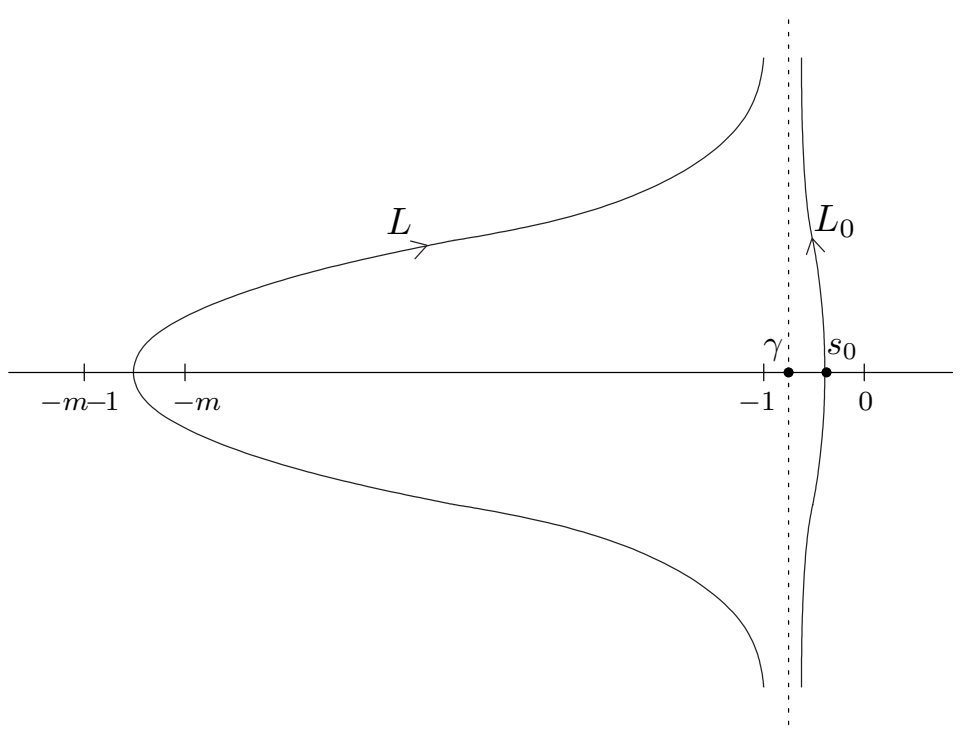

Figure 6.2.1 
Then, we consider the following Mellin-Barnes type integrals:

$$
\begin{aligned}
\Phi_{m}^{+}(t) & :=\frac{1}{2 \pi \sqrt{-1}} \int_{L_{0}} \frac{\Gamma(-\lambda)}{\Gamma(\lambda+1+m)}(2 t)_{+}^{\lambda} d \lambda, \\
\Psi_{m}^{+}(t) & :=\frac{1}{2 \pi \sqrt{-1}} \int_{L} \frac{\Gamma(-\lambda)}{\Gamma(\lambda+1+m)}(2 t)_{+}^{\lambda} d \lambda, \\
\Phi_{m}(t) & :=\frac{1}{2 \pi \sqrt{-1}} \int_{L_{0}} \frac{\Gamma(-\lambda)}{\Gamma(\lambda+1+m)}\left(\frac{(2 t)_{+}^{\lambda}}{\tan (\pi \lambda)}+\frac{(2 t)_{-}^{\lambda}}{\sin (\pi \lambda)}\right) d \lambda, \\
\Psi_{m}(t) & :=\frac{1}{2 \pi \sqrt{-1}} \int_{L} \frac{\Gamma(-\lambda)}{\Gamma(\lambda+1+m)}\left(\frac{(2 \lambda)_{+}^{\lambda}}{\tan (\pi \lambda)}+\frac{(2 t)_{-}^{\lambda}}{\sin (\pi \lambda)}\right) d \lambda .
\end{aligned}
$$

We shall see that these integrals are special cases of (6.1.8) and define distributions on $\mathbb{R}$. The next theorem is the main result of this section, which will be derived from Proposition 6.1 .2 by applying the reduction formula of Meijer's $G$-functions.

Theorem 6.2.1. 1) $\Phi_{m}^{+}(t)$ and $\Phi_{m}(t)$ are locally integrable functions on $\mathbb{R}$. Furthermore, for a sufficiently small $\epsilon>0, \Phi_{m}^{+}(t)|t|^{-\epsilon}$ and $\Phi_{m}(t)|t|^{-\epsilon}$ are also locally integrable.

2)

$$
\begin{aligned}
& \Psi_{m}^{+}(t)=\Phi_{m}^{+}(t)-\sum_{k=1}^{m} \frac{(-1)^{k-1}}{2^{k}(m-k) !} \delta^{(k-1)}(t) . \\
& \Psi_{m}(t)=\Phi_{m}(t)-\frac{1}{\pi} \sum_{k=1}^{m} \frac{(k-1) !}{2^{k}(m-k) !} t^{-k} .
\end{aligned}
$$

See (7.1.5) in Appendix for the definition of the distribution $t^{-k}$. In particular, $\Psi_{m}^{+}$and $\Psi_{m}$ are defined as functionals on the space $C_{0}^{m-1}(\mathbb{R})$ of compactly supported functions on $\mathbb{R}$ with continuous derivatives up to $m-1$ if $m \geq 1$.

The rest of this section is devoted to the proof of Theorem 6.2.1.

Before regarding the integrals (6.2.1) $-(6.2 .4)$ as those for distributions, we consider the classic cases, namely, their restrictions to $\mathbb{R} \backslash\{0\}$, which are real analytic functions.

Let $L_{i}(i=1,2,3)$ be contours that start at $\gamma_{i}-\sqrt{-1} \infty$ and end at 
$\gamma_{i}+\sqrt{-1} \infty$, and pass the real axis at some point $s_{i}$. We assume

$$
\begin{array}{ll}
-\frac{m}{2}<\gamma_{1}, & s_{1}<0, \\
-\frac{m}{2}<\gamma_{2}, & s_{2}<-m, \\
& s_{3}<-m .
\end{array}
$$

Then, we have the following integral expressions of Bessel functions. Although the results are classical, we shall give a proof to illustrate the idea of passing from Bessel functions to Bessel distributions. The proof below is based on the integral expressions of Meijer's $G$-functions (see Appendix 7.6, see also Proposition $6.1 .2(2))$ :

Lemma 6.2.2. 1) For $t>0$,

$$
\begin{aligned}
\widetilde{J}_{m}(2 \sqrt{2 t}) & =(2 t)^{-\frac{m}{2}} J_{m}(2 \sqrt{2 t}) \\
& =\frac{1}{2 \pi \sqrt{-1}} \int_{L_{1}} \frac{\Gamma(-\lambda)}{\Gamma(\lambda+m+1)}(2 t)_{+}^{\lambda} d \lambda .
\end{aligned}
$$

2) For $t>0$,

$$
\begin{aligned}
\tilde{Y}_{m}(2 \sqrt{2 t}) & =(2 t)^{-\frac{m}{2}} Y_{m}(2 \sqrt{2 t}) \\
& =\frac{1}{2 \pi \sqrt{-1}} \int_{L_{2}} \frac{\Gamma(-\lambda)}{\Gamma(\lambda+m+1)} \frac{(2 t)_{+}^{\lambda}}{\tan (\pi \lambda)} d \lambda .
\end{aligned}
$$

3) For $t<0$,

$$
\begin{aligned}
\widetilde{K}_{m}(2 \sqrt{2|t|}) & =(2|t|)^{-\frac{m}{2}} K_{m}(2 \sqrt{2|t|}) \\
& =\frac{(-1)^{m+1}}{4 \sqrt{-1}} \int_{L_{3}} \frac{\Gamma(-\lambda)}{\Gamma(\lambda+m+1)} \frac{(2 t)_{-}^{\lambda}}{\sin (\pi \lambda)} d \lambda .
\end{aligned}
$$

Proof of Lemma 6.2.2. Each of the first equalities is by the definition of the normalized Bessel functions $\widetilde{J}_{m}, \widetilde{Y}_{m}$, and $\widetilde{K}_{m}$ given in (7.2.3), (7.2.5), and (7.2.6), respectively. Let us verify the second equalities (the integral formulas for the Bessel functions).

1) By the reduction formula (7.6.12) of the $G$-function $G_{02}^{10}$, we have

$$
(2 t)^{-\frac{m}{2}} J_{m}(2 \sqrt{2 t})=G_{02}^{10}(2 t \mid 0,-m)
$$

for $t>0$. Then, by the integral expression (7.6.2) of the $G$-function $G_{02}^{10}$, we have

$$
G_{02}^{10}(2 t \mid 0,-m)=\frac{1}{2 \pi \sqrt{-1}} \int_{L_{1}} \frac{\Gamma(-\lambda)}{\Gamma(1+m+\lambda)}(2 t)^{\lambda} d \lambda
$$


for $t>0$. Hence, (6.2.7) is proved.

2) By the reduction formula (7.6.15) of the $G$-function $G_{13}^{20}$, we have

$$
(2 t)^{-\frac{m}{2}} Y_{m}(2 \sqrt{2 t})=G_{13}^{20}\left(\begin{array}{l|c}
2 t & \begin{array}{c}
-m-\frac{1}{2} \\
-m, 0,-m-\frac{1}{2}
\end{array}
\end{array}\right),
$$

for $t>0$. Then, by Example 7.6.2, we have

$$
\begin{aligned}
& G_{13}^{20}\left(\begin{array}{l|c}
2 t & -m-\frac{1}{2} \\
-m, 0,-m-\frac{1}{2}
\end{array}\right) \\
& =\frac{1}{2 \pi \sqrt{-1}} \int_{L_{2}} \frac{\Gamma(-m-\lambda) \Gamma(-\lambda)}{\Gamma\left(m+\frac{3}{2}+\lambda\right) \Gamma\left(-m-\frac{1}{2}-\lambda\right)}(2 t)^{\lambda} d \lambda .
\end{aligned}
$$

Now, (6.2.8) is deduced from this formula and the following identity:

$$
\frac{\Gamma(-m-\lambda) \Gamma(\lambda+1+m)}{\Gamma\left(m+\frac{3}{2}+\lambda\right) \Gamma\left(-m-\frac{1}{2}-\lambda\right)}=\frac{1}{\tan \pi \lambda} \quad \text { for any } m \in \mathbb{Z}
$$

Here, the last identity is an elementary consequence of the formula $\Gamma(z) \Gamma(1-$ $z)=\frac{\pi}{\sin \pi z}$.

3) By the reduction formula (7.6.13) of the $G$-function $G_{02}^{20}$, we have

$$
(2|t|)^{-\frac{m}{2}} K_{m}(2 \sqrt{2|t|})=\frac{1}{2} G_{02}^{20}(2|t| \mid 0,-m) .
$$

Suppose $t<0$. Then, again by the integral expression (7.6.2) of $G_{02}^{20}$, the right-hand side amounts to

$$
\frac{1}{4 \pi \sqrt{-1}} \int_{L_{3}} \Gamma(-\lambda) \Gamma(-m-\lambda)(2 t)_{-}^{\lambda} d \lambda .
$$

Then, (6.2.9) follows from the identity:

$$
\Gamma(\lambda+1+m) \Gamma(-m-\lambda)=\frac{\pi}{\sin (-\pi(\lambda+m))}=\frac{(-1)^{m+1} \pi}{\sin \pi \lambda} .
$$

Thus, all the statements of Lemma 6.2 .2 are proved.

The integrals in Lemma 6.2 .2 do not depend on the choice of $L_{i}(i=$ $1,2,3)$ as ordinary functions on $\mathbb{R} \backslash\{0\}$. However, as we mentioned in Remark 6.1.3, they depend on the choice of $L_{i}$ as distributions on $\mathbb{R}$ because the poles of the distributions $t_{ \pm}^{\lambda}$ are located at $\lambda=-1,-2,-3, \ldots$ 
To avoid this effect, we need to impose more constraints on the contours $L_{i}$. Thus, let us assume further $-1<s_{1}$ and $-m-1<s_{i}(i=2,3)$. Moreover, we assume $-1<\gamma_{j}(j=1,2)$. That is, we shall assume from now that the integral paths $L_{i}(i=1,2,3)$ are under the following constraints:

$$
\begin{aligned}
-1<\gamma_{1}, & -1 & <s_{1} & <0, \\
-1<\gamma_{2}, & -m-1 & <s_{2} & <-m, \\
& -m-1 & <s_{3} & <-m .
\end{aligned}
$$

Then, the right-hand sides of (6.2.7) -(6.2.9) define distributions on $\mathbb{R}$, which are independent of the choice of the integral paths $L_{i}(i=1,2,3)$ subject to $(6.2 .10)-(6.2 .12)$.

Proof of Theorem 6.2.1. The first statement is a special case of Proposition 6.1.2 (3).

Let us show the second statement. The contour $L$ used in (6.2.2) and (6.2.4) meets the constraints (6.2.11) and (6.2.12), and can be used as $L_{2}$ and $L_{3}$. Likewise, the contour $L_{0}$ used in (6.2.1) and (6.2.3) can be used as $L_{1}$. Further, we shall assume that the contour $L_{0}$ coincides with $L$ when $|\operatorname{Im} \lambda|$ is sufficiently large.

The integrand of (6.2.1) has poles at $\lambda=-1,-2, \ldots,-m$ inside the closed contour $L_{0}-L$, and its residue is given by

$$
\underset{\lambda=-k}{\operatorname{res}} \frac{\Gamma(-\lambda)}{\Gamma(\lambda+1+m)}(2 t)_{+}^{\lambda}=\frac{(-1)^{k-1}}{2^{k}(m-k) !} \delta^{(k-1)}(t)
$$

for $k=1,2, \ldots, m$ by (7.1.1). Therefore, by Cauchy's integral formula, we have

$$
\begin{aligned}
& \frac{1}{2 \pi \sqrt{-1}}\left(\int_{L_{0}}-\int_{L}\right) \frac{\Gamma(-\lambda)}{\Gamma(\lambda+1+m)}(2 t)_{+}^{\lambda} d \lambda \\
& =\sum_{k=1}^{m} \frac{(-1)^{k-1}}{2^{k}(m-k) !} \delta^{(k-1)}(t)
\end{aligned}
$$

as distributions. Hence, (6.2.5) is proved.

Next, let us prove (6.2.6). We recall from (7.1.3) that the Laurent expansion of the distribution $t_{ \pm}^{\lambda}$ at $\lambda=-k(k=1,2, \ldots)$ is given by

$$
\begin{aligned}
t_{+}^{\lambda} & =\frac{1}{\lambda+k} \frac{(-1)^{k-1}}{(k-1) !} \delta^{(k-1)}(t)+t_{+}^{-k}+\cdots \\
t_{-}^{\lambda} & =\frac{1}{(\lambda+k)(k-1) !} \delta^{(k-1)}(t)+t_{-}^{-k}+\cdots
\end{aligned}
$$


Combining with the Taylor expansions at $\lambda=-k(k=1,2, \ldots, m)$ :

$$
\begin{aligned}
\sin \pi \lambda & =(-1)^{k} \pi(\lambda+k)+\cdots, \\
\tan \pi \lambda & =\pi(\lambda+k)+\cdots, \\
\frac{\Gamma(-\lambda) 2^{\lambda}}{\Gamma(\lambda+1+m)} & =b_{0}+b_{1}(\lambda+k)+\cdots,
\end{aligned}
$$

where $b_{0}=\frac{(k-1) !}{2^{k}(m-k) !}$, we have

$$
\begin{aligned}
& \frac{\Gamma(-\lambda)}{\Gamma(\lambda+1+m)}\left(\frac{(2 t)_{+}^{\lambda}}{\tan (\pi \lambda)}+\frac{(2 t)_{-}^{\lambda}}{\sin (\pi \lambda)}\right) \\
= & \frac{b_{0}\left((-1)^{k-1} \delta^{(k-1)}(t)+(-1)^{k} \delta^{(k-1)}(t)\right)}{\pi(k-1) !} \frac{1}{(\lambda+k)^{2}} \\
& +\left(\frac{b_{0}\left(t_{+}^{-k}+(-1)^{k} t_{-}^{-k}\right)}{\pi}+\frac{b_{1}\left((-1)^{k-1} \delta^{(k-1)}(t)+(-1)^{k} \delta^{(k-1)}(t)\right)}{\pi(k-1)^{2}}\right) \frac{1}{\lambda+k}+\cdots \\
= & \frac{(k-1) ! t^{-k}}{2^{k}(m-k) ! \pi} \frac{1}{\lambda+k}+O(1),
\end{aligned}
$$

as $\lambda$ tends to $-k$.

Therefore, by (6.2.3) and (6.2.4), we have

$$
\begin{aligned}
\Psi_{m}(t)-\Phi_{m}(t) & =\frac{1}{2 \pi \sqrt{-1}}\left(\int_{L}-\int_{L_{0}}\right) \frac{\Gamma(-\lambda)}{\Gamma(\lambda+1+m)}\left(\frac{(2 t)_{+}^{\lambda}}{\tan (\pi \lambda)}+\frac{(2 t)_{-}^{\lambda}}{\sin (\pi \lambda)}\right) d \lambda \\
& =-\sum_{k=1}^{m} \frac{(k-1) !}{2^{k}(m-k) ! \pi} t^{-k} .
\end{aligned}
$$

Hence, (6.2.6) is proved. Now, we have completed the proof of Theorem 6.2.1.

Remark 6.2.3 (Bessel distributions). We shall use the symbols

$$
\begin{aligned}
& \widetilde{J}_{m}\left(2 \sqrt{2 t_{+}}\right)=(2 t)_{+}^{-\frac{m}{2}} J_{m}\left(2 \sqrt{2 t_{+}}\right), \\
& \widetilde{K}_{m}\left(2 \sqrt{2 t_{+}}\right)=(2 t)_{+}^{-\frac{m}{2}} K_{m}\left(2 \sqrt{2 t_{+}}\right), \\
& \widetilde{Y}_{m}\left(2 \sqrt{2 t_{-}}\right)=(2 t)_{-}^{-\frac{m}{2}} Y_{m}\left(2 \sqrt{2 t_{-}}\right),
\end{aligned}
$$

to denote the distributions defined by the right-hand sides of $(6.2 .7)-(6.2 .9)$ and by the contours $L_{i}(i=1,2,3)$ satisfying (6.2.10)-(6.2.12), respectively. 
It is noteworthy that $\widetilde{J}_{m}\left(2 \sqrt{2 t_{+}}\right)$is a locally integrable function on $t \in \mathbb{R}$ in view of the Taylor expansion (7.2.3), but $\widetilde{K}_{m}\left(2 \sqrt{2 t_{+}}\right)$and $\widetilde{Y}_{m}\left(2 \sqrt{2 t_{-}}\right)$are not (see (7.2.8) and (7.2.9) ). Then, by the above proof of Theorem 6.2.1, we have

$$
\begin{aligned}
\Phi_{m}^{+}(t) & =\widetilde{J}_{m}\left(2 \sqrt{2 t_{+}}\right) \\
& =(2 t)_{+}^{-\frac{m}{2}} J_{m}\left(2 \sqrt{2 t_{+}}\right) \\
\Psi_{m}^{+}(t) & =\widetilde{J}_{m}\left(2 \sqrt{2 t_{+}}\right)-\sum_{k=1}^{m} \frac{(-1)^{k-1}}{2^{k}(m-k) !} \delta^{(k-1)}(t) \\
& =(2 t)_{+}^{-\frac{m}{2}} J_{m}\left(2 \sqrt{2 t_{+}}\right)-\sum_{k=1}^{m} \frac{(-1)^{k-1}}{2^{k}(m-k) !} \delta^{(k-1)}(t), \\
\Psi_{m}(t) & =\widetilde{Y}_{m}\left(2 \sqrt{2 t_{+}}\right)+\frac{2(-1)^{m+1}}{\pi} \widetilde{K}_{m}\left(2 \sqrt{2 t_{-}}\right) \\
& =(2 t)_{+}^{-\frac{m}{2}} Y_{m}\left(2 \sqrt{2 t_{+}}\right)+\frac{2(-1)^{m+1}}{\pi}(2 t)_{-}^{-\frac{m}{2}} K_{m}\left(2 \sqrt{2 t_{-}}\right) .
\end{aligned}
$$

An alternative way to define the distributions $\widetilde{K}_{m}\left(2 \sqrt{2 t_{+}}\right)$and $\widetilde{Y}_{m}\left(2 \sqrt{2 t_{-}}\right)$ is to use the infinite sum expressions (7.2.8) and (7.2.9). Let $t_{ \pm}^{-k}$ be the regularized distributions given by the second terms in (7.1.3) and (7.1.4). In light of (7.2.8) and (7.2.9), we define as distributions.

$$
\begin{aligned}
\widetilde{Y}_{m}\left(2 \sqrt{2 t_{+}}\right)= & -\frac{1}{\pi} \sum_{k=1}^{m} \frac{(k-1) !}{2^{k}(m-k) !} t_{+}^{-k}+\frac{1}{\pi} \widetilde{J}_{m}\left(2 \sqrt{2 t_{+}}\right) \log \left(2 t_{+}\right) \\
& -\frac{1}{\pi} \sum_{l=0}^{\infty}(-1)^{l}\left(2 t_{+}\right)^{l} \frac{\psi(m+l+1)+\psi(l+1)}{l !(m+l) !} \\
\frac{2(-1)^{m+1}}{\pi} \widetilde{K}_{m}\left(2 \sqrt{2 t_{-}}\right)= & \frac{-1}{\pi} \sum_{k=1}^{m} \frac{(-1)^{k}(k-1) !}{2^{k}(m-k) !} t_{-}^{-k}+\frac{1}{\pi} \widetilde{I}_{m}\left(2 \sqrt{2 t_{-}}\right) \log \left(2 t_{-}\right) \\
& -\frac{1}{\pi} \sum_{l=0}^{\infty}\left(2 t_{-}\right)^{l} \frac{\psi(m+l+1)+\psi(l+1)}{l !(m+l) !} .
\end{aligned}
$$

If we set

$$
\begin{aligned}
\Phi_{m}(t):= & \frac{-1}{\pi} \sum_{l=0}^{\infty}(-2 t)^{l} \frac{\psi(m+l+1)+\psi(l+1)}{l !(m+l) !} \\
& +\frac{1}{\pi}\left(\widetilde{J}_{m}\left(2 \sqrt{2 t_{+}}\right) \log \left(2 t_{+}\right)+\widetilde{I}_{m}\left(2 \sqrt{2 t_{-}}\right) \log (2 t-1)\right),
\end{aligned}
$$


then $\Phi_{m}(t)$ is a locally integrable function, and

$$
\widetilde{Y}_{m}\left(2 \sqrt{2 t_{+}}\right)+\frac{2(-1)^{m+1}}{\pi} \widetilde{K}_{m}\left(2 \sqrt{2 t_{-}}\right)=\phi_{m}(t)-\frac{1}{\pi} \sum_{k=1}^{m} \frac{(k-1) !}{2^{k}(m-k) !} t^{-k} .
$$

This gives another explanation of (6.2.6). In this book, we have adopted the integral expression (6.2.3) for the definition of $\Phi_{m}(t)$ in place of the expansion (6.2.19).

\subsection{Differential equations for Bessel distributions}

The kernel $K\left(x, x^{\prime}\right)$ of the unitary inversion operator $\pi\left(w_{0}\right)$ is given by means of the Bessel distribution. In this section, we shall give a heuristic account on why the Bessel function arises in the kernel function.

We begin with the observation that generic $L_{+}$-orbits on $C \times C$ are of codimension one (see Lemma 6.3.2). This would force that the kernel function of any $L_{+}$-intertwining operator (see (2.5.6) for the definition of $L_{+}=M_{+}^{\max } A$ ) should be a function of one variable $\left\langle x, x^{\prime}\right\rangle$. The second step is to make use of the differential equation arising from $\operatorname{Ad}\left(w_{0}\right) \mathfrak{n}^{\max }=\overline{\mathfrak{n}^{\max }}$ (see (2.5.4)). Since $\mathfrak{n}^{\max }$ acts on $L^{2}(C)^{\infty}$ as differential operators of second order, we get a differential equation of second order that the kernel distribution $K\left(x, x^{\prime}\right)$ must satisfy (see Proposition 6.3.4). The technical point here is that we have avoided using the Casimir operator of $K$ because it acts on $L^{2}(C)^{\infty}$ as a differential operator of fourth order.

The argument here was the clue for us to find a coarse form of the kernel $K\left(x, x^{\prime}\right)$. Though we did not use the results of this section for the actual proof of our main theorem, we think that the idea here is still helpful to get a coarse solution to a similar problem (see Problem 1.8.1 (2)) in other settings, namely, to find the integral kernel of the unitary inversion operator $\pi\left(w_{0}\right)$ of the minimal representation of other groups.

Let $\theta: g \mapsto{ }^{t} g^{-1}$ be the Cartan involution of $G$. Since $g \in G=O(p, q)$ satisfies ${ }^{t} g I_{p, q} g=I_{p, q}$ where $I_{p, q}=\left(\begin{array}{cc}I_{p} & 0 \\ 0 & -I_{q}\end{array}\right)$, we have ${ }^{t} g^{-1}=I_{p, q} g I_{p, q}^{-1}$. Since $w_{0}=I_{p, q}$, we get

$$
\theta(g)=w_{0} g w_{0}^{-1} .
$$

We let $L_{+}=M_{+}^{\max } A$ act on $C$ by

$$
m e^{t H} \cdot x=e^{t} m x
$$

for $m \in M_{+}^{\max } \simeq O(p-1, q-1)$ and $a=e^{t E} \in A$ (see Section 2.3 for notation). 
Lemma 6.3.1. The kernel function $K\left(x, x^{\prime}\right)$ of the unitary operator $\pi\left(w_{0}\right)$ satisfies the following functional equation:

$$
K\left(x, x^{\prime}\right)=K\left(\theta(l) x, l x^{\prime}\right) \quad \text { for all } l \in L_{+} .
$$

Proof. Building on the unitary representation $\left(\pi, L^{2}(C)\right)$, we define another unitary representation $\pi^{\theta}$ on $L^{2}(C)$ by the following twist:

$$
\pi^{\theta}(g):=\pi(\theta(g))
$$

Then, (6.3.1) implies that $\pi\left(w_{0}\right): L^{2}(C) \rightarrow L^{2}(C)$ is an intertwining operator from $\left(\pi, L^{2}(C)\right)$ to $\left(\pi^{\theta}, L^{2}(C)\right)$. In particular, we have,

$$
\pi^{\theta}(l) \circ \pi\left(w_{0}\right)=\pi\left(w_{0}\right) \circ \pi(l) \quad \text { for any } l \in L_{+} .
$$

For $l=m \in M_{+}^{\max }$, we recall from (2.3.6) that

$$
(\pi(m) u)(x)=u\left({ }^{t} m x\right) \quad \text { for } x \in C .
$$

Hence, for any $u \in L^{2}(C)$, we have

$$
\int_{C} K\left({ }^{t} \theta(m) x, x^{\prime}\right) u\left(x^{\prime}\right) d \mu\left(x^{\prime}\right)=\int_{C} K\left(x, x^{\prime \prime}\right) u\left({ }^{t} m x^{\prime \prime}\right) d \mu\left(x^{\prime \prime}\right) .
$$

Since $d \mu$ is $M_{+}^{\max }$-invariant, the right-hand side is equal to

$$
\int_{C} K\left(x,{ }^{t} m^{-1} x^{\prime}\right) u\left(x^{\prime}\right) d \mu\left(x^{\prime}\right) .
$$

Since $u$ is arbitrary, the kernel function must coincide:

$$
K\left({ }^{t} \theta(m) x, x^{\prime}\right)=K\left(x,{ }^{t} m^{-1} x^{\prime}\right) .
$$

Replacing ${ }^{t} \theta(m) x$ with $x$, we have

$$
K\left(x, x^{\prime}\right)=K\left(m x,{ }^{t} m^{-1} x^{\prime}\right) \quad \text { for any } m \in M_{+}^{\max } .
$$

Thus, (6.3.2) holds for $l \in M_{+}^{\max }$. For $l=a:=e^{t H} \in A$, we recall from (2.3.12) that

$$
(\pi(a) u)(x)=e^{-\frac{p+q-4}{2} t} u\left(e^{-t} x\right)(x \in C) .
$$

Since $\pi^{\theta}(a)=\pi\left(a^{-1}\right)$, the equation (6.3.3) amounts to

$$
\pi\left(w_{0}\right)=\pi(a) \circ \pi\left(w_{0}\right) \circ \pi(a) .
$$


Hence, for any $u \in L^{2}(C)$, we have

$$
\int_{C} K\left(x, x^{\prime}\right) u\left(x^{\prime}\right) d \mu\left(x^{\prime}\right)=e^{-(p+q-4) t} \int_{C} K\left(e^{-t} x, x^{\prime \prime}\right) u\left(e^{-t} x^{\prime \prime}\right) d \mu\left(x^{\prime \prime}\right) .
$$

By the formula (2.2.3) of the measure $d \mu$ in the polar coordinate, we have

$$
d \mu\left(x^{\prime \prime}\right)=e^{(p+q-4) t} d \mu\left(x^{\prime}\right) \text { for } x^{\prime}=e^{-t} x^{\prime \prime} .
$$

Thus, the right-hand side equals $\int_{C} K\left(e^{-t} x, e^{t} x^{\prime}\right) u\left(x^{\prime}\right) d \mu\left(x^{\prime}\right)$. Hence, we have

$$
K\left(x, x^{\prime}\right)=K\left(e^{-t} x, e^{t} x^{\prime}\right) \quad \text { for any } t \in \mathbb{R}
$$

and therefore

$$
K\left(x, x^{\prime}\right)=K\left(\theta(a) x, a x^{\prime}\right) \quad \text { for any } a \in A .
$$

Now, Lemma 6.3.1 is proved.

Now let $M_{+}^{\max }$ act on the direct product manifold $C \times C$ by the formula:

$$
M_{+}^{\max } \times C \times C \rightarrow C \times C, \quad\left(l, x, x^{\prime}\right) \mapsto\left(\theta(l) x, l x^{\prime}\right) .
$$

Furthermore, we define the level set of $C \times C$ by

$$
H_{t}:=\left\{\left(x, x^{\prime}\right) \in C \times C:\left\langle x, x^{\prime}\right\rangle=t\right\} \quad \text { for } t \in \mathbb{R} .
$$

Here, $\langle\cdot, \cdot\rangle$ is the standard positive definite inner product on $\mathbb{R}^{p+q-2}$. Then we have:

Lemma 6.3.2. 1) The level set $H_{t}$ is stable under the $M_{+}^{\max }$-action.

2) Moreover $H_{t}$ is a single $M_{+}^{\max }$-orbit for any non-zero $t$.

Proof. 1) For $x, x^{\prime} \in \mathbb{R}^{p+q-2}\left(\subset \mathbb{R}^{p+q}\right)$ and $l \in M_{+}^{\max } \simeq O(p-1, q-1)$, we have

$$
\begin{aligned}
\left\langle\theta(l) x, l x^{\prime}\right\rangle & =\left\langle w_{0} l w_{0}^{-1} x, l x^{\prime}\right\rangle \\
& ={ }^{t} x^{\prime} l w_{0} l w_{0}^{-1} x \\
& ={ }^{t} x^{\prime} w_{0} w_{0}^{-1} x \\
& =\left\langle x, x^{\prime}\right\rangle .
\end{aligned}
$$

Hence, $H_{t}$ is $M_{+}^{\max }$-stable.

2 ) We replace $(p-1, q-1)$ by $(p, q)$, and consider the $G$-action on

$$
\widetilde{C}:=\left\{\left(x_{0}, \cdots, x_{p+q-1}\right): x_{0}^{2}+\cdots+x_{p-1}^{2}-x_{p}^{2}-\cdots-x_{p+q-1}^{2}=0\right\}
$$


in place of the $M_{+}^{\max }$-action on $C$ (this change allows us to use the notation $N^{\max }$ and $\overline{N^{\max }}$ in Section 2.3$)$. Then, we recall from (2.3.8) that $G$ acts transitively on $\widetilde{C}$ and the isotropy subgroup at $e_{0}+e_{p+q-1}={ }^{t}(1,0, \cdots, 0,1)$ is given by $M_{+}^{\max } N^{\max }\left(\operatorname{see}(2.3 .8)\right.$ ). For $t \neq 0$, we take any $\left(x, x^{\prime}\right) \in H_{t}$. Then, we find $g_{1} \in G$ such that $g_{1} x^{\prime}={ }^{t}(1,0, \ldots, 0,1)$. We write $\theta\left(g_{1}\right) x=$ ${ }^{t}\left(x_{0}, x, x_{p+q-1}\right) \in \mathbb{R} \oplus \mathbb{R}^{p+q-2} \oplus \mathbb{R}$. Then,

$$
t=\left\langle x, x^{\prime}\right\rangle=\left\langle\theta\left(g_{1}\right) x, g_{1} x^{\prime}\right\rangle=x_{0}+x_{p+q-1} .
$$

Let us consider the orbit of $\theta\left(M_{+}^{\max } N^{\max }\right)=M_{+}^{\max } \bar{N}^{\max }$ on $\widetilde{C}$. In view of $(2.3 .6)$, we have

$$
\bar{n}_{b}\left(\begin{array}{c}
x_{0} \\
x \\
x_{p+q-1}
\end{array}\right)=\left(\begin{array}{c}
x_{0}-{ }^{t} x w_{0} b \\
x \\
x_{p+q-1}+{ }^{t} x w_{0} b
\end{array}\right)+\frac{x_{0}+x_{p+q-1}}{2}\left(\begin{array}{c}
-Q(b) \\
2 b \\
Q(b)
\end{array}\right),
$$

for $b={ }^{t}\left(b_{1}, \cdots, b_{p+q-2}\right)$ and $x={ }^{t}\left(x_{1}, \cdots, x_{p+q-2}\right) \in \mathbb{R}^{p+q-2}$. If $x_{0}+$ $x_{p+q-1} \neq 0$ and ${ }^{t}\left(x_{0}, \cdots, x_{p+q-1}\right) \in \widetilde{C}$, we set

$$
b:=\frac{-x}{x_{0}+x_{p+q-1}} .
$$

Since $x_{0}^{2}-x_{p+q-1}^{2}=-Q(x)$, we have

$$
b=\frac{x_{0}-x_{p+q-1}}{Q(x)} x \quad \text { and } \quad Q(b)=\frac{x_{0}-x_{p+q-1}}{x_{0}+x_{p+q-1}} .
$$

Then, we have

$$
\bar{n}_{b}\left(\begin{array}{c}
x_{0} \\
x \\
x_{p+q-1}
\end{array}\right)=\left(\begin{array}{c}
x_{0} \\
0 \\
x_{p+q-1}
\end{array}\right)+\frac{Q(x)}{2\left(x_{0}+x_{p+q-1}\right)}\left(\begin{array}{c}
1 \\
0 \\
-1
\end{array}\right)=\frac{x_{0}+x_{p+q-1}}{2}\left(\begin{array}{l}
1 \\
0 \\
1
\end{array}\right) .
$$

Now, we set $g:=\theta\left(\bar{n}_{b}\right) g_{1}$. Then,

$$
\left(\theta(g) x, g x^{\prime}\right)=\left(\bar{n}_{b} \theta\left(g_{1}\right) x, \theta\left(\bar{n}_{b}\right) g_{1} x^{\prime}\right)=\left(\frac{t}{2}\left(\begin{array}{l}
1 \\
0 \\
1
\end{array}\right),\left(\begin{array}{l}
1 \\
0 \\
1
\end{array}\right)\right) .
$$

Hence, the second statement is proved. 6.3 .2

Thus, we have the following proposition by Lemma 6.3.1 and Lemma 
Proposition 6.3.3. $\left.K\left(x, x^{\prime}\right)\right|_{C \times C \backslash H_{0}}$ is of the form

$$
K\left(x, x^{\prime}\right)=\Psi\left(\left\langle x, x^{\prime}\right\rangle\right)
$$

for some function $\Psi(t)$ defined on $\mathbb{R} \backslash\{0\}$.

By lifting the inversion relation $\operatorname{Ad}\left(w_{0}\right) \mathfrak{n}^{\max }=\overline{\mathfrak{n}^{\max }}$ (see (2.5.4)$)$ in the Lie algebra $\mathfrak{g}$ to the actions on $L^{2}(C)_{K}$, we get the differential equation satisfied by $\Psi$. More precisely,

Proposition 6.3.4. $\Psi(t)$ satisfies the following ordinary differential equation on $\mathbb{R} \backslash\{0\}$ :

$$
t \frac{d^{2} \Psi}{d t^{2}}+\frac{p+q-4}{2} \frac{d \Psi}{d t}+2 \Psi=0 .
$$

Proof. It follows from $\operatorname{Ad}\left(w_{0}\right) \bar{N}_{j}=\epsilon_{j} N_{j}$ (see (2.5.3)) that

$$
\pi\left(w_{0}\right) \circ d \pi\left(\bar{N}_{j}\right)=\epsilon_{j} d \pi\left(N_{j}\right) \circ \pi\left(w_{0}\right) .
$$

We recall from Sections 2.3 and 2.4 that

$$
\begin{array}{llrl}
d \pi\left(\bar{N}_{j}\right) & =2 \sqrt{-1} x_{j} & & (\text { see }(2.3 .13)), \\
d \pi\left(N_{j}\right) & =\frac{\sqrt{-1}}{2} \epsilon_{j} P_{j} & & (\text { see }(2.3 .19)),
\end{array}
$$

where $P_{j}$ are the fundamental differential operators on the isotropic cone $C$ such that

$$
P_{j} \psi=\left.\left(\epsilon_{j} x_{j} \square-(2 E+p+q-4) \frac{\partial}{\partial x_{j}}\right) \tilde{\psi}\right|_{C}
$$

if $\psi=\left.\tilde{\psi}\right|_{C}$ for a function defined in a neighborhood of $C$. Then, (6.3.7) leads us to the functional equation for any test function $u\left(x^{\prime}\right)$ :

$$
4 \int_{C} \Psi\left(\left\langle x, x^{\prime}\right\rangle\right) x_{j}^{\prime} u\left(x^{\prime}\right) d \mu\left(x^{\prime}\right)=\int_{C}\left(P_{j} \Psi\left(\left\langle x, x^{\prime}\right\rangle\right)\right) u\left(x^{\prime}\right) d \mu\left(x^{\prime}\right) .
$$

Now, in view of (6.3.8),

$$
\begin{aligned}
P_{j} \Psi\left(\left\langle x, x^{\prime}\right\rangle\right)= & \epsilon_{j} x_{j} Q\left(x^{\prime}\right) \Psi^{\prime \prime}\left(\left\langle x, x^{\prime}\right\rangle\right) \\
& -x_{j}^{\prime}\left(2\left\langle x, x^{\prime}\right\rangle \Psi^{\prime \prime}\left(\left\langle x, x^{\prime}\right\rangle\right)+(p+q-4) \Psi^{\prime}\left(\left\langle x, x^{\prime}\right\rangle\right)\right) \\
= & -\left.x_{j}^{\prime}\left(2 t \Psi^{\prime \prime}(t)+(p+q-4) \Psi^{\prime}(t)\right)\right|_{t=\left\langle x, x^{\prime}\right\rangle} .
\end{aligned}
$$

Hence, the functional equation (6.3.9) for any $u \in L^{2}(C)$ implies that $\Psi$ satisfies the following differential equation:

$$
4 \Psi(t)=-\left(2 t \Psi^{\prime \prime}(t)+(p+q-4) \Psi^{\prime}(t)\right) .
$$

Thus, Proposition 6.3 .4 is proved. 
We write $\theta=t \frac{d}{d t}$. As $\theta^{2}=t^{2} \frac{d t^{2}}{d t}+t \frac{d}{d t},(6.3 .6)$ is equivalent to

$$
\left(\theta^{2}+\frac{p+q-6}{2} \theta+2 t\right) \Psi(t)=0 .
$$

Finally, let us see directly the differential equations that the Bessel distributions $\Phi_{m}^{+}(t), \Psi_{m}^{+}(t)$, and $\Psi_{m}(t)$ (see (6.2.1), (6.2.2), and (6.2.4) for definition) satisfy. It is easy to see that $\Phi_{m}^{+}(t), \Psi_{m}^{+}(t)$, and $\Psi_{m}(t)$ solve the following differential equation (in an ordinary sense)

$$
\left(\theta^{2}+m \theta+2 t\right) u=0 \quad \text { on } \mathbb{R} \backslash\{0\} .
$$

Of course, this fits well with what Proposition 6.3 .4 asserts. On the other hand, the distribution $\Phi_{m}(t)$ (see (6.2.3) for definition) does not appear in the kernel function $K\left(x, x^{\prime}\right)$. We note that, as distributions,

$$
\begin{aligned}
& \left(\theta^{2}+2 m \theta+2 t\right) \sum_{k=1}^{m} \frac{(-1)^{k-1}}{2^{k}(m-k) !} \delta^{(k-1)}(t)=0, \\
& \left(\theta^{2}+2 m \theta+2 t\right) \sum_{k=1}^{m} \frac{(k-1) !}{2^{k}(m-k) !} t^{-k}=\frac{1}{(m-1) !} .
\end{aligned}
$$

In particular, $\Phi_{m}(t)$ (see (6.2.3) ) does not solve (6.3.10), but solves the third order differential equation on $\mathbb{R} \backslash\{0\}$ :

$$
\theta\left(\theta^{2}+2 m \theta+2 t\right) \Phi_{m}(t)=0
$$




\section{Chapter 7}

\section{Appendix: special functions}

We have seen that various special functions arise naturally in the analysis on the minimal representations. Some of their fundamental properties (e.g. integral formulas, differential equations, etc.) have been used in the proof of the unitary inversion formulas. Conversely, representation theoretic properties are reflected as algebraic relations (e.g. functional equations) of such special functions. Further, different models of the same representation yield functional equations connecting special functions arising from each model.

For the convenience of the reader, we collect the formulas and the properties of special functions that were used in the previous chapters.

\subsection{Riesz distribution $x_{+}^{\lambda}$}

A distribution $f_{\lambda}$ on $\mathbb{R}$ with parameter $\lambda \in \mathbb{C}$ is said to be a distribution with meromorphic parameter $\lambda$ if the pairing

$$
\left\langle f_{\lambda}, \varphi\right\rangle
$$

is a meromorphic function of $\lambda$ for any test function $\varphi \in C_{0}^{\infty}(\mathbb{R})$. We say $f_{\lambda}$ has a pole at $\lambda=\lambda_{0}$ if $\left\langle f_{\lambda}, \varphi\right\rangle$ has a pole at $\lambda=\lambda_{0}$ for some $\varphi$. Then, taking a residue at $\lambda=\lambda_{0}$, we get a distribution:

$$
C_{0}^{\infty}(\mathbb{R}) \rightarrow \mathbb{C}, \quad \varphi \mapsto \underset{\lambda=\lambda_{0}}{\operatorname{res}}\left\langle f_{\lambda}, \varphi\right\rangle,
$$

which we denote by $\underset{\lambda=\lambda_{0}}{\operatorname{res}} f_{\lambda}$.

By Cauchy's integral formula, if $C$ is a contour surrounding $\lambda=\lambda_{0}$, then we have

$$
\underset{\lambda=\lambda_{0}}{\operatorname{res}}\left\langle f_{\lambda}, \varphi\right\rangle=\frac{1}{2 \pi \sqrt{-1}} \int_{C}\left\langle f_{\lambda}, \varphi\right\rangle d \lambda
$$


and in turn we get an identity of distributions:

$$
\underset{\lambda=\lambda_{0}}{\operatorname{res}} f_{\lambda}=\frac{1}{2 \pi \sqrt{-1}} \int_{C} f_{\lambda} d \lambda
$$

A classic example of distributions with meromorphic parameter is the Riesz distribution $x_{+}^{\lambda}$ defined as a locally integrable function (and hence a distribution):

$$
x_{+}^{\lambda}= \begin{cases}x^{\lambda} & (x>0) \\ 0 & (x \leq 0)\end{cases}
$$

for $\lambda \in \mathbb{C}$ such that $\operatorname{Re} \lambda>-1$. Then, $x_{+}^{\lambda}$ extends meromorphically to the entire complex plane, and all the poles are located at $\lambda=-1,-2, \ldots$ The residue is given by

$$
\underset{\lambda=-k}{\operatorname{res}} x_{+}^{\lambda}=\frac{(-1)^{k-1}}{(k-1) !} \delta^{(k-1)}(x)
$$

for $k=1,2,3, \ldots$ Here, $\delta(x)$ is the Dirac delta function. To see this, we set

$$
\varphi_{N}(x):=\varphi(x)-\sum_{k=1}^{N} \frac{\varphi^{(k-1)}(0)}{(k-1) !} x^{k-1} .
$$

Then,

$$
\begin{aligned}
\left\langle x_{+}^{\lambda}, \varphi\right\rangle= & \sum_{k=1}^{N} \frac{\varphi^{(k-1)}(0)}{(k-1) !} \int_{0}^{1} x^{\lambda+k-1} d x+\int_{0}^{1} x^{\lambda} \varphi_{N}(x) d x \\
& +\int_{1}^{\infty} x^{\lambda} \varphi(x) d x \\
= & \sum_{k=1}^{N} \frac{1}{\lambda+k} \frac{\varphi^{(k-1)}(0)}{(k-1) !}+\int_{0}^{1} x^{\lambda} \varphi_{N}(x) d x \\
& +\int_{1}^{\infty} x^{\lambda} \varphi(x) d x .
\end{aligned}
$$

The first two terms have a simple pole at $\lambda=-k$ with residue

$$
\frac{\varphi^{(k-1)}(0)}{(k-1) !}=\left\langle\frac{(-1)^{k-1}}{(k-1) !} \delta^{(k-1)}(x), \varphi(x)\right\rangle
$$

the second term is holomorphic if $\operatorname{Re} \lambda>-N-1$ because $\varphi_{N}(x)=O\left(x^{N}\right)$, and the last term is an entire function of $\lambda$ because $\varphi$ is compactly supported. 
Hence, (7.1.1) is proved. Likewise,

$$
x_{-}^{\lambda}:= \begin{cases}|x|^{\lambda} & (x<0) \\ 0 & (x \geq 0)\end{cases}
$$

extends a distribution with meromorphic parameter $\lambda$ and all the poles are located at $\lambda=0,-1,-2, \ldots$. They are simple poles with

$$
\operatorname{res}_{\lambda=-k} x_{-}^{\lambda}=\frac{\delta^{(k-1)}(x)}{(k-1) !} \text {. }
$$

We write the Laurent expansions of $x_{+}^{\lambda}$ and $x_{-}^{\lambda}$ at $\lambda=-k(k=1,2,3, \ldots)$ as follows:

$$
\begin{aligned}
& x_{+}^{\lambda}=\frac{(-1)^{k-1}}{\lambda+k} \delta^{(k-1)}(x)+x_{+}^{-k}+(\lambda+k) x_{+}^{-k} \log x_{+}+\cdots, \\
& x_{-}^{\lambda}=\frac{1}{\lambda+k} \delta^{(k-1)}(x)+x_{-}^{-k}+(\lambda+k) x_{-}^{-k} \log x_{-}+\cdots .
\end{aligned}
$$

Then, $x_{+}^{-k}$ and $x_{-}^{-k}$ are tempered distributions supported on the half lines $x \geq 0$ and $x \leq 0$, respectively. We note that they are not homogeneous as distributions. Then, the sum $x_{+}^{\lambda}+(-1)^{\lambda} x_{-}^{\lambda}$ becomes a distribution with holomorphic parameter $\lambda$ in the entire complex plane because

$$
\underset{\lambda=-k}{\operatorname{res}}\left(x_{+}^{\lambda}+(-1)^{\lambda} x_{-}^{\lambda}\right)=0
$$

for $k=1,2,3, \ldots$. We now define a distribution

$$
x^{-k}:=\left.\left(x_{+}^{\lambda}+(-1)^{\lambda} x_{-}^{\lambda}\right)\right|_{\lambda=-k} .
$$

This distribution is homogeneous, and coincides with $x_{+}^{-k}+(-1)^{k} x_{-}^{k}$.

For $k=1, x^{-1}$ is the distribution that gives Cauchy's principal value:

$$
\left\langle x^{-1}, \varphi\right\rangle=\lim _{\epsilon \downarrow 0}\left(\int_{-\infty}^{-\epsilon}+\int_{\epsilon}^{\infty}\right) \frac{\varphi(x)}{x} d x .
$$

This formula is valid for any $\varphi \in C_{0}(\mathbb{R})$. Likewise, $x^{-k}$ extends to a functional on the space $C_{0}^{k-1}(\mathbb{R})$ of compactly supported functions on $\mathbb{R}$ with continuous derivatives up to $k-1$. See the textbook [24] of Gelfand and Shilov for a nice introduction to these distributions. 


\subsection{Bessel functions $J_{\nu}, I_{\nu}, K_{\nu}, Y_{\nu}$}

For $\operatorname{Re} \nu>0$, the series

$$
J_{\nu}(z):=\left(\frac{z}{2}\right)^{\nu} \sum_{j=0}^{\infty} \frac{(-1)^{j}\left(\frac{z}{2}\right)^{2 j}}{j ! \Gamma(j+\nu+1)}
$$

converges in the entire complex plane. Its sum $J_{\nu}(z)$ is called the Bessel function of the first kind of order $\nu$ (see [79, §3.54]). $J_{\nu}(z)$ extends meromorphically to $\nu \in \mathbb{C}$ by the Poisson integration formula:

$$
J_{\nu}(z)=\frac{1}{\sqrt{\pi} \Gamma\left(\nu+\frac{1}{2}\right)}\left(\frac{z}{2}\right)^{\nu} \int_{-1}^{1} e^{\sqrt{-1} z t}\left(1-t^{2}\right)^{\nu-\frac{1}{2}} d t
$$

and solves the Bessel differential equation:

$$
\left(z^{2} \frac{d^{2}}{d z^{2}}+z \frac{d}{d z}+\left(z^{2}-\nu^{2}\right)\right) u=0
$$

We set

$$
Y_{\nu}(z):=\frac{J_{\nu}(z) \cos \nu \pi-J_{-\nu}(z)}{\sin \nu \pi} .
$$

If $\nu$ is an integer, say $\nu=m$, then this definition reads as

$$
Y_{m}(z):=\lim _{\nu \rightarrow m} \frac{J_{\nu}(z) \cos \nu \pi-J_{-\nu}(z)}{\sin \nu \pi} .
$$

$Y_{\nu}$ is known as the Bessel function of the second kind or Neumann's function.

Further, we define two more functions by

$$
\begin{aligned}
I_{\nu}(z) & :=e^{-\frac{\sqrt{-1} \nu \pi}{2}} J_{\nu}\left(e^{\frac{\sqrt{-1} \pi}{2}} z\right) \\
& =\left(\frac{z}{2}\right)^{\nu} \sum_{j=0}^{\infty} \frac{\left(\frac{z}{2}\right)^{2 j}}{j ! \Gamma(j+\nu+1)}, \\
K_{\nu}(z) & :=\frac{\pi}{2 \sin \nu \pi}\left(I_{-\nu}(z)-I_{\nu}(z)\right) .
\end{aligned}
$$

Both of them solve the following differential equation:

$$
z^{2} \frac{d^{2} u}{d z^{2}}+z \frac{d u}{d z}-\left(z^{2}+\nu^{2}\right) u=0 .
$$

$I_{\nu}(z)$ is known as the modified Bessel function of the first kind, and is real when $\nu \in \mathbb{R}$ and $z>0$. 
$K_{\nu}(z)$ is known as the modified Bessel function of the third kind or Basset's function. Clearly we have

$$
K_{-\nu}(z)=K_{\nu}(z) .
$$

We call $J_{\nu}, Y_{\nu}, I_{\nu}$, and $K_{\nu}$ simply as J-Bessel, $Y$-Bessel, I-Bessel, and $K$-Bessel functions.

The $K$-Bessel function satisfies the following formula (see [14, II, $\S 7.11$ $(22)])$ :

$$
\left(\frac{d}{z d z}\right)^{m}\left(z^{-\nu} K_{\nu}(z)\right)=(-1)^{m} z^{-\nu-m} K_{\nu+m}(z) .
$$

This formula may be stated as

$$
\left(-\frac{2 d}{z d z}\right)^{m} \widetilde{K}_{\nu}(z)=\widetilde{K}_{\nu+m}(z)
$$

in terms of the normalized $K$-Bessel function (7.2.6). By the change of variables $z=2 e^{-x}$, the $m=1$ case amounts to:

$$
\begin{aligned}
\frac{d}{d x}\left(e^{-a x} \widetilde{K}_{\nu}\left(2 e^{-x}\right)\right)= & -a e^{-a x} \widetilde{K}_{\nu}\left(2 e^{-x}\right) \\
& +2 e^{-(a+2) x} \widetilde{K}_{\nu+1}\left(2 e^{-x}\right) .
\end{aligned}
$$

The $K$-Bessel functions $K_{\nu}(z)$ reduce to combinations of elementary functions if $\nu$ is half of an odd integer. For $n \in \mathbb{N}$ we have

$$
\begin{aligned}
K_{n+\frac{1}{2}}(z) & =\left(\frac{\pi}{2 z}\right)^{\frac{1}{2}} e^{-z} \sum_{j=0}^{n} \frac{(n+j) !}{j !(n-j) !} \frac{1}{(2 z)^{j}} \\
& =(-1)^{n}\left(\frac{\pi}{2 z}\right)^{\frac{1}{2}} z^{n+1}\left(\frac{d}{z d z}\right)^{n} \frac{e^{-z}}{z} .
\end{aligned}
$$

For instance, if $n=0$, we have

$$
K_{\frac{1}{2}}(z)=\left(\frac{\pi}{2 z}\right)^{\frac{1}{2}} e^{-z}
$$

The following renormalization is sometimes convenient:

$$
\begin{aligned}
\widetilde{J}_{\nu}(z) & :=\left(\frac{z}{2}\right)^{-\nu} J_{\nu}(z)=\sum_{j=0}^{\infty} \frac{(-1)^{j}\left(\frac{z}{2}\right)^{2 j}}{j ! \Gamma(\nu+j+1)} \\
\widetilde{I}_{\nu}(z) & :=\left(\frac{z}{2}\right)^{-\nu} I_{\nu}(z)=\sum_{j=0}^{\infty} \frac{\left(\frac{z}{2}\right)^{2 j}}{j ! \Gamma(j+\nu+1)} \\
\widetilde{Y}_{\nu}(z) & :=\left(\frac{z}{2}\right)^{-\nu} Y_{\nu}(z) \\
\widetilde{K}_{\nu}(z) & :=\left(\frac{z}{2}\right)^{-\nu} K_{\nu}(z) .
\end{aligned}
$$


By the Taylor expansion as above, we see that both $\widetilde{J}_{\nu}(z)$ and $\widetilde{I}_{\nu}(z)$ are holomorphic function of $z$ in the entire complex plane.

$\widetilde{J}_{\nu}(z)$ and $\widetilde{Y}_{\nu}(z)$ are linearly independent of each other (whether $\nu$ is an integer or not) and form a basis of the space of solutions to the following differential equation:

$$
z \frac{d^{2} u}{d z^{2}}+(2 \nu+1) \frac{d u}{d z}+z u=0
$$

or equivalently,

$$
\left(\theta^{2}+2 \nu \theta+z^{2}\right) u=0,
$$

where $\theta:=z \frac{d}{d z}$. On the other hand, $\widetilde{I}_{\nu}(z)$ and $\widetilde{K}_{\nu}(z)$ solves

$$
\left(\theta^{2}+2 \nu \theta-z^{2}\right) u=0 .
$$

In terms of Meijer's $G$-functions (Appendix 7.6) or the Barnes hypergeometric function ${ }_{p} F_{q}$ (see $(\mathbf{7 . 6 . 8})$ ), we shall have the following expressions: (see $(7.6 .12)-(7.6 .15))$ :

$$
\begin{aligned}
\widetilde{J}_{\nu}(z) & =G_{02}^{10}\left(\frac{z^{2}}{4} \mid 0,-\nu\right)=\frac{1}{\Gamma(\nu+1)}{ }_{0} F_{1}\left(\nu+1 ;-\frac{z^{2}}{4}\right), \\
\widetilde{Y}_{\nu}(z) & =G_{13}^{20}\left(\frac{z^{2}}{4} \mid \begin{array}{c}
-\nu-\frac{1}{2} \\
-\nu, 0,-\nu-\frac{1}{2}
\end{array}\right), \\
\widetilde{K}_{\nu}(z) & =\frac{1}{2} G_{02}^{20}\left(\frac{z^{2}}{4} \mid 0,-\nu\right) .
\end{aligned}
$$

The Mellin-Barnes type integral expression of $\widetilde{J}_{\nu}(z), \widetilde{Y}_{\nu}(z)$, and $\widetilde{K}_{\nu}(z)$ is also given in Lemma 6.2.2,

For $m=1,2,3, \ldots$, the infinite sum expressions of $Y_{m}(z)$ and $K_{m}(z)$ (or $\widetilde{Y}_{m}(z)$ and $\left.\widetilde{K}_{m}(z)\right)$ at $z=0$ are given in [14, II, $\S 7.2,(31)$ and (37)], which 
may be stated as follows:

$$
\begin{aligned}
\widetilde{Y}_{m}(z)= & -\frac{1}{\pi} \sum_{k=1}^{m}\left(\frac{z}{2}\right)^{-2 k} \frac{(k-1) !}{(m-k) !} \\
& +\frac{2}{\pi} \widetilde{J}_{m}(z) \log \left(\frac{z}{2}\right) \\
& -\frac{1}{\pi} \sum_{l=0}^{\infty}(-1)^{l}\left(\frac{z}{2}\right)^{2 l} \frac{\psi(m+l+1)+\psi(l+1)}{l !(m+l) !} . \\
\widetilde{K}_{m}(z)= & \frac{1}{2} \sum_{k=1}^{m}(-1)^{m-k}\left(\frac{z}{2}\right)^{-2 k} \frac{(k-1) !}{(m-k) !} \\
& +(-1)^{m+1} \widetilde{I}_{m}(z) \log \left(\frac{z}{2}\right) \\
& +\frac{1}{2}(-1)^{m} \sum_{l=0}^{\infty}\left(\frac{z}{2}\right)^{2 l} \frac{\psi(m+l+1)+\psi(l+1)}{l !(m+l) !} .
\end{aligned}
$$

Here, the function $\psi(z)$ is the logarithmic derivative of the gamma function:

$$
\psi(z):=\frac{d \log \Gamma(z)}{d z}=\frac{\Gamma^{\prime}(z)}{\Gamma(z)} .
$$

The $\psi$ function is meromorphic with simple poles at $z=0,-1,-2, \ldots$.

Next, we summarize the asymptotic behaviors of the Bessel functions:

Fact 7.2.1 (see [1, Chapter 4], [79, Chapter VII]). The asymptotic behaviors of the Bessel functions at $z=0, \infty$ are given by

1) As z tends to $0, J_{\nu}(z), I_{\nu}(z)=O\left(z^{\nu}\right)$.

For $\nu>0$,

$$
\widetilde{K}_{\nu}(z)=\frac{\Gamma(\nu)}{2}\left(\frac{z}{2}\right)^{-2 \nu}+o\left(z^{-2 \nu}\right) \quad \text { as } z \rightarrow 0 .
$$


2) As z tends to infinity

$$
\begin{aligned}
& J_{\nu}(z) \sim \sqrt{\frac{2}{\pi z}}\left(\cos \left(z-\frac{\nu \pi}{2}-\frac{\pi}{4}\right) \sum_{j=0}^{\infty} \frac{(-1)^{j}(\nu, 2 j)}{(2 z)^{2 j}}\right. \\
& \left.-\sin \left(z-\frac{\nu \pi}{2}-\frac{\pi}{4}\right) \sum_{j=0}^{\infty} \frac{(-1)^{j}(\nu, 2 j+1)}{(2 z)^{2 j+1}}\right) \quad(|\arg z|<\pi), \\
& Y_{\nu}(z) \sim \sqrt{\frac{2}{\pi z}}\left(\sin \left(z-\frac{\nu \pi}{2}-\frac{\pi}{4}\right) \sum_{j=0}^{\infty}(-1)^{j} \frac{(\nu, 2 j)}{(2 z)^{2 j}}\right. \\
& \left.+\cos \left(z-\frac{\nu \pi}{2}-\frac{\pi}{4}\right) \sum_{j=0}^{\infty}(-1)^{j} \frac{(\nu, 2 j+1)}{(2 z)^{2 j+1}}\right) \quad(|\arg z|<\pi), \\
& I_{\nu}(z) \sim \frac{e^{z}}{\sqrt{2 \pi z}} \sum_{j=0}^{\infty} \frac{(-1)^{j}(\nu, j)}{(2 z)^{j}}+\frac{e^{-z+\left(\nu+\frac{1}{2}\right) \sqrt{-1} \pi}}{\sqrt{2 \pi z}} \sum_{j=0}^{\infty} \frac{(\alpha, j)}{(2 z)^{j}} \quad\left(-\frac{\pi}{2}<\arg z<\frac{3}{2} \pi\right), \\
& K_{\nu}(z) \sim \sqrt{\frac{\pi}{2 z}} e^{-z}\left(1+\sum_{j=1}^{\infty} \frac{(\nu, j)}{(2 z)^{j}}\right) \quad\left(|\arg z|<\frac{3 \pi}{2}\right) .
\end{aligned}
$$

In particular, we have

$$
\widetilde{K}_{\nu}(2 z)=\frac{\sqrt{\pi}}{2} e^{-2 z} z^{-\nu-\frac{1}{2}}\left(1+O\left(\frac{1}{z}\right)\right) \quad \text { as } z \rightarrow \infty .
$$

Here, we have used Hankel's notation:

$$
\begin{aligned}
(\alpha, j) & :=(-1)^{j} \frac{\left(\frac{1}{2}-\alpha\right)_{j}\left(\frac{1}{2}+\alpha\right)_{j}}{j !} \\
& =\frac{\left(4 \alpha^{2}-1^{2}\right)\left(4 \alpha^{2}-3^{2}\right) \cdots\left(4 \alpha^{2}-(2 j-1)^{2}\right)}{2^{2 j} j !} .
\end{aligned}
$$

Finally, we list some integral formulas for the Bessel functions:

B1 (the Mellin transform of $K$-Bessel functions, see [26, p. 684]). For $\operatorname{Re}(\mu+$ $1 \pm \nu)>0$ and $\operatorname{Re} a>0$

$$
\int_{0}^{\infty} t^{\mu} K_{\nu}(a t) d t=2^{\mu-1} a^{-\mu-1} \Gamma\left(\frac{1+\mu+\nu}{2}\right) \Gamma\left(\frac{1+\mu-\nu}{2}\right) .
$$

Equivalently, we have

$$
\int_{0}^{\infty} t^{s} \widetilde{K}_{\nu}(a t) d t=2^{s-1} a^{-s-1} \Gamma\left(\frac{1+s}{2}\right) \Gamma\left(\frac{1+s}{2}-\nu\right) .
$$


B2 Formula of the Hankel transform due to W. Bailey [3] (see also [15, $\S 19.6(8)])$.

$$
\begin{aligned}
\int_{0}^{\infty} & t^{\lambda-1} J_{\mu}(a t) J_{\nu}(b t) K_{\rho}(c t) d t \\
= & \frac{2^{\lambda-2} a^{\mu} b^{\nu} \Gamma\left(\frac{1}{2}(\lambda+\mu+\nu-\rho)\right) \Gamma\left(\frac{1}{2}(\lambda+\mu+\nu+\rho)\right)}{c^{\lambda+\mu+\nu} \Gamma(\mu+1) \Gamma(\nu+1)} \\
& \quad \times F_{4}\left(\frac{1}{2}(\lambda+\mu+\nu-\rho), \frac{1}{2}(\lambda+\mu+\nu+\rho) ; \mu+1, \nu+1 ;-\frac{a^{2}}{c^{2}},-\frac{b^{2}}{c^{2}}\right) .
\end{aligned}
$$

Here, $F_{4}$ is Appell's hypergeometric function of two variables (see (7.7.4)).

B3 (see [15, $\S 7,14.2(36)])$ For $\operatorname{Re}(\alpha+\beta)>0$ and $\operatorname{Re}(\rho \pm \mu \pm \nu+1)>0$,

$$
\begin{aligned}
& 2^{\rho+2} \Gamma(1-\rho) \int_{0}^{\infty} K_{\mu}(\alpha t) K_{\nu}(\beta t) t^{-\rho} d t \\
& =\alpha^{\rho-\nu-1} \beta_{2}^{\nu} F_{1}\left(\frac{1+\nu+\mu-\rho}{2}, \frac{1+\nu-\mu-\rho}{2} ; 1-\rho ; 1-\frac{\beta^{2}}{\alpha^{2}}\right) \\
& \times \Gamma\left(\frac{1+\nu+\mu-\rho}{2}\right) \Gamma\left(\frac{1+\nu-\mu-\rho}{2}\right) \Gamma\left(\frac{1-\nu+\mu-\rho}{2}\right) \Gamma\left(\frac{1-\nu-\mu-\rho}{2}\right) .
\end{aligned}
$$

In particular, we have

$$
\int_{0}^{\infty} K_{\mu}(2 t)^{2} t^{2 s-1} d t=\frac{\Gamma(s)^{2} \Gamma(s+\mu) \Gamma(s-\mu)}{8 \Gamma(2 s)} .
$$

\subsection{Associated Legendre functions $P_{\nu}^{\mu}$}

The associated Legendre functions on the interval $(-1,1)$ is defined as the special value of the hypergeometric function:

$$
P_{\nu}^{\mu}(x)=\frac{1}{\Gamma(1-\mu)}\left(\frac{1+x}{1-x}\right)^{\frac{\mu}{2}}{ }_{2} F_{1}\left(-\nu, \nu+1 ; 1-\mu ; \frac{1-x}{2}\right) .
$$

The associated Legendre functions satisfy the following functional relation:

$$
\frac{d}{d x}\left(\left(1-x^{2}\right)^{-\frac{\mu}{2}} P_{\nu}^{\mu}(-x)\right)=\left(1-x^{2}\right)^{-\frac{\mu+1}{2}} P_{\nu}^{\mu+1}(-x),
$$

which is derived from the following recurrence relation (see [26, $§ 8.733$ (1)]):

$$
\left(1-x^{2}\right) \frac{d}{d x} P_{\nu}^{\mu}(x)=-\sqrt{1-x^{2}} P_{\nu}^{\mu+1}(x)-\mu x P_{\nu}^{\mu}(x) .
$$

Integral formulas for the associated Legendre functions: 
L1 (see [26, p. 803]) Formula of the Riemann-Liouville integral: $\operatorname{Re} \lambda<1$, $\operatorname{Re} \mu>0,0<y<1$,

$$
\frac{1}{\Gamma(\mu)} \int_{0}^{y}(y-x)^{\mu-1}(x(1-x))^{-\frac{\lambda}{2}} P_{\nu}^{\lambda}(1-2 x) d x=(y(1-y))^{\frac{\mu}{2}-\frac{\lambda}{2}} P_{\nu}^{\lambda-\mu}(1-2 y) .
$$

L2 (see [26, p. 798]) For $2 \operatorname{Re} \lambda>|\operatorname{Re} \mu|$,

$$
\int_{-1}^{1}\left(1-x^{2}\right)^{\lambda-1} P_{\nu}^{\mu}(x) d x=\frac{\pi 2^{\mu} \Gamma\left(\lambda+\frac{\mu}{2}\right) \Gamma\left(\lambda-\frac{\mu}{2}\right)}{\Gamma\left(\lambda+\frac{\nu}{2}+\frac{1}{2}\right) \Gamma\left(\lambda-\frac{\nu}{2}\right) \Gamma\left(\frac{-\mu+\nu+2}{2}\right) \Gamma\left(\frac{-\mu-\nu+1}{2}\right)} .
$$

\subsection{Gegenbauer polynomials $C_{l}^{\mu}$}

Definition of the Gegenbauer polynomials: For $l \in \mathbb{N}$, we define

$$
C_{l}^{\mu}(x):=\frac{(-1)^{l}}{2^{l}} \frac{\Gamma(2 \mu+l) \Gamma\left(\mu+\frac{1}{2}\right)}{\Gamma(2 \mu) \Gamma\left(\mu+l+\frac{1}{2}\right)} \frac{\left(1-x^{2}\right)^{\frac{1}{2}-\mu}}{l !} \frac{d^{l}}{d x^{l}}\left(\left(1-x^{2}\right)^{\mu+l-\frac{1}{2}}\right) .
$$

Slightly different from the usual notation in the literature, we adopt the following normalization of the Gegenbauer polynomial:

$$
\widetilde{C}_{l}^{\mu}(x):=\Gamma(\mu) C_{l}^{\mu}(x) .
$$

By using Gauss's duplication formula

$$
\Gamma(2 \mu)=2^{2 \mu-1} \pi^{-\frac{1}{2}} \Gamma(\mu) \Gamma\left(\mu+\frac{1}{2}\right),
$$

the definition (7.4.1) may be stated as

$$
\widetilde{C}_{l}^{\mu}(x)=\frac{(-1)^{l} \Gamma(2 \mu+l) \sqrt{\pi}}{2^{2 \mu+l-1} l ! \Gamma\left(\mu+l+\frac{1}{2}\right)}\left(1-x^{2}\right)^{-\mu+\frac{1}{2}} \frac{d^{l}}{d x^{l}}\left(\left(1-x^{2}\right)^{\mu+l-\frac{1}{2}}\right) .
$$

The special value at $\mu=0$ is given by the limit formula (see [14, $§ 3.15 .1$ $(14)])$ :

$$
\widetilde{C}_{l}^{0}(\cos \theta)=\lim _{\mu \rightarrow 0} \Gamma(\mu) C_{l}^{\mu}(\cos \theta)=\frac{2 \cos (l \theta)}{l} .
$$

On the other hand, the special value at $l=0$ is given by

$$
\widetilde{C}_{0}^{\mu}(x)=\Gamma(\mu) .
$$


Connection with Gauss's hypergeometric function (see [14, §3.15 (3)]):

$$
\begin{aligned}
\widetilde{C}_{l}^{\mu}(x) & =\frac{\Gamma(l+2 \mu) \Gamma(\mu)}{\Gamma(l+1) \Gamma(2 \mu)}{ }_{2} F_{1}\left(l+2 \mu,-l ; \mu+\frac{1}{2} ; \frac{1-x}{2}\right) \\
& =\frac{\Gamma(l+2 \mu) \Gamma(\mu)}{\Gamma(l+1) \Gamma(2 \mu)}{ }_{2} F_{1}\left(\frac{l+2 \mu}{2},-\frac{l}{2} ; \mu+\frac{1}{2} ; 1-x^{2}\right) .
\end{aligned}
$$

Here, the second equation is derived from the formula of quadratic transformation for hypergeometric function (see [14, §2.11 (2)]):

$$
{ }_{2} F_{1}\left(a, b ; a+b+\frac{1}{2} ; 4 x(1-x)\right)={ }_{2} F_{1}\left(2 a, 2 b ; a+b+\frac{1}{2} ; x\right) .
$$

By using Kummer's transformation formula for the hypergeometric functions:

$$
{ }_{2} F_{1}(\alpha, \beta ; \gamma ; z)=(1-z)^{\gamma-\alpha-\beta}{ }_{2} F_{1}(\gamma-\alpha, \gamma-\beta ; \gamma ; z),
$$

one can obtain the following relationship between the Gegenbauer polynomials and the associated Legendre functions.

$$
\widetilde{C}_{l}^{\mu}(x)=\frac{\sqrt{\pi} \Gamma(2 \mu+l)}{2^{\mu-\frac{1}{2}} \Gamma(l+1)}\left(1-x^{2}\right)^{\frac{1}{4}-\frac{\mu}{2}} P_{\mu+l-\frac{1}{2}}^{\frac{1}{2}-\mu}(x), \quad-1<x<1 .
$$

Integral formulas for the Gegenbauer polynomials:

Ge1 (Orthogonality relations; see [14, $\S 3.15 .1(17)]$ ) For $\operatorname{Re} \mu>-\frac{1}{2}$,

$$
\int_{-1}^{1} \widetilde{C}_{l}^{\mu}(x) \widetilde{C}_{m}^{\mu}(x)\left(1-x^{2}\right)^{\mu-\frac{1}{2}} d x= \begin{cases}0 & \text { if } l \neq m, \\ \frac{2^{1-2 \mu} \pi \Gamma(l+2 \mu)}{(l+\mu) \Gamma(l+1)} & \text { if } l=m .\end{cases}
$$

Ge2 (see [26, §7.321]) For $\operatorname{Re} \mu>-\frac{1}{2}$,

$$
\int_{-1}^{1}\left(1-x^{2}\right)^{\mu-\frac{1}{2}} e^{\sqrt{-1} a x} \widetilde{C}_{l}^{\mu}(x) d x=\frac{\pi 2^{1-\mu} \Gamma(2 \mu+l)}{\Gamma(l+1)} a^{-\mu} J_{\mu+l}(a) .
$$

Ge3 (see [46, Lemma 8.5.2]) For $\alpha \in \mathbb{C}, \operatorname{Re} \nu>-1$, and $l \in \mathbb{N}$,

$$
\begin{aligned}
\int_{-1}^{1} J_{\nu}(\alpha \sqrt{x+1}) & \widetilde{C}_{l}^{\nu+\frac{1}{2}}(x)(1+x)^{\frac{\nu}{2}}(1-x)^{\nu} d x= \\
& \frac{2^{\frac{3}{2}}(-1)^{l} \sqrt{\pi} \Gamma(2 \nu+l+1)}{\alpha^{\nu+1} l !} J_{2 \nu+2 l+1}(\sqrt{2} \alpha) .
\end{aligned}
$$


Ge4 For $\operatorname{Re} \nu>-\frac{1}{2}$ and $\operatorname{Re} \lambda>-1$,

$$
\begin{aligned}
& \int_{-x}^{1}(x+y)^{\lambda} \widetilde{C}_{k}^{\nu}(y)\left(1-y^{2}\right)^{\nu-\frac{1}{2}} d y \\
& =\frac{\sqrt{\pi} \Gamma(2 \nu+k) \Gamma(\lambda+1)}{2^{\nu-\frac{1}{2}} k !}\left(1-x^{2}\right)^{\frac{\lambda}{2}+\frac{\nu}{2}+\frac{1}{4}} P_{\nu+k-\frac{1}{2}}^{-\lambda-\nu-\frac{1}{2}}(-x) .
\end{aligned}
$$

This formula (7.4.11) is essentially the integration formula (7.3.3) for the associated Legendre functions. For the sake of completeness, we give a proof:

The left-hand side of (7.4.11)

$$
\begin{aligned}
& =\frac{\sqrt{\pi} \Gamma(2 \nu+k)}{2^{\nu-\frac{1}{2}} k !} \int_{-x}^{1}\left(1-y^{2}\right)^{\frac{\nu}{2}-\frac{1}{4}}(x+y)^{\lambda} P_{\nu+k-\frac{1}{2}}^{\frac{1}{2}-\nu}(y) d y \quad \text { by (7.4.7) } \\
& =\frac{2^{\lambda+1} \sqrt{\pi} \Gamma(2 \nu+k)}{k !} \int_{0}^{\frac{1+x}{2}}((1-t) t)^{\frac{\nu}{2}-\frac{1}{4}}\left(\frac{x+1}{2}-t\right)^{\lambda} P_{\nu+k-\frac{1}{2}}^{\frac{1}{2}-\nu}(1-2 t) d t \\
& =\frac{2^{\lambda+1} \sqrt{\pi} \Gamma(2 \nu+k) \Gamma(\lambda+1)}{k !}\left(\frac{1-x^{2}}{4}\right)^{\frac{\lambda}{2}+\frac{\nu}{2}+\frac{1}{4}} P_{\nu+k-\frac{1}{2}}^{-\lambda-\nu-\frac{1}{2}}(-x) \quad \text { by }(7.3 .3 \\
& =\text { the right-hand side of (7.4.11). }
\end{aligned}
$$

\subsection{Spherical harmonics $\mathcal{H}^{j}\left(\mathbb{R}^{m}\right)$ and branching laws}

A spherical harmonics $f$ of degree $j=0,1,2, \ldots$ is the restriction to the unit sphere $S^{m-1} \subset \mathbb{R}^{m}$ of a homogeneous harmonic polynomials of degree $j$ in $\mathbb{R}^{m}$. Equivalently, $f$ is a smooth function satisfying the differential equation:

$$
\Delta_{S^{m-1}} f=-j(j+m-2) f .
$$

The space of spherical harmonics of degree $j$ is denoted by

$$
\mathcal{H}^{j}\left(\mathbb{R}^{m}\right):=\left\{f \in C^{\infty}\left(S^{m-1}\right): \Delta_{S^{m-1}} f=-j(j+m-2) f\right\} .
$$

When $m=1$, it is convenient to set:

$$
\mathcal{H}^{0}\left(\mathbb{R}^{1}\right):=\mathbb{C} 1, \quad \mathcal{H}^{1}\left(\mathbb{R}^{1}\right):=\mathbb{C} \operatorname{sgn}, \quad \mathcal{H}^{j}\left(\mathbb{R}^{1}\right):=0 \quad(j \geq 2) .
$$

The following facts are well-known (see [31, Introduction], 69]):

H1 For $f \in \mathcal{H}^{j}\left(\mathbb{R}^{m}\right), f(-x)=(-1)^{j} f(x)$. 
H2 $O(m)$ acts irreducibly on $\mathcal{H}^{j}\left(\mathbb{R}^{m}\right)$.

H3 $\mathcal{H}^{j}\left(\mathbb{R}^{m}\right)$ is still irreducible as an $S O(m)$-module if $m \geq 3$.

H4 $\mathcal{H}^{j}\left(\mathbb{R}^{2}\right)=\mathbb{C} e^{\sqrt{-1} j \theta} \oplus \mathbb{C} e^{-\sqrt{-1} j \theta}, j \geq 1$ as $S O(2)$-modules, where $\theta=$ $\tan ^{-1} \frac{y}{x},(x, y) \in \mathbb{R}^{2}$.

H5 $\left.\mathcal{H}^{j}\left(\mathbb{R}^{m}\right)\right|_{O(m-1)} \simeq \bigoplus_{i=0}^{j} \mathcal{H}^{i}\left(\mathbb{R}^{m-1}\right)$ as $O(m-1)$-modules.

H6 The Hilbert space $L^{2}\left(S^{m-1}\right)$ decomposes into a direct sum of the space of spherical harmonics:

$$
L^{2}\left(S^{m-1}\right) \simeq \sum_{j=0}^{\infty} \mathcal{H}^{j}\left(\mathbb{R}^{m}\right) .
$$

Here, $\sum^{\oplus}$ stands for the Hilbert completion of the algebraic direct $\operatorname{sum} \bigoplus_{j=0}^{\infty} \mathcal{H}^{j}\left(\mathbb{R}^{m}\right)$.

Let $\left(x_{0}, x\right) \in \mathbb{R}^{m}, x \in \mathbb{R}^{m-1}$ be a coordinate of $\mathbb{R}^{m}$. Then, the branching law $\mathbf{H 5}$ is explicitly constructed by the $O(m-1)$-intertwining operator

$$
I_{i \rightarrow j}^{m}: \mathcal{H}^{i}\left(\mathbb{R}^{m-1}\right) \rightarrow \mathcal{H}^{j}\left(\mathbb{R}^{m}\right)
$$

as follows (see [69, Chapter III]):

Fact 7.5.1. For $0 \leq i \leq j$ and $\phi \in \mathcal{H}^{i}\left(\mathbb{R}^{m-1}\right)$, we define a function $I_{i \rightarrow j}^{m} \phi$ on $S^{m-1}$ by

$$
\left(I_{i \rightarrow j}^{m}(\phi)\right)\left(x_{0}, x\right):=|x|^{i} \phi\left(\frac{x}{|x|}\right) \widetilde{C}_{j-i}^{\frac{m-2}{2}+i}\left(x_{0}\right) .
$$

Here, $\widetilde{C}_{l}^{\nu}(z)$ is the normalized Gegenbauer polynomial (see (7.4.2)). Then,

1) $I_{i \rightarrow j}^{m}(\phi) \in \mathcal{H}^{j}\left(\mathbb{R}^{m}\right)$.

2) $I_{i \rightarrow j}^{m}$ gives an injective $O(m-1)$-homomorphism from $\mathcal{H}^{i}\left(\mathbb{R}^{m-1}\right)$ to $\mathcal{H}^{j}\left(\mathbb{R}^{m}\right)$.

3) ( $L^{2}$-norm)

$$
\left\|I_{i \rightarrow j}^{m}(\phi)\right\|_{L^{2}\left(S^{m-1}\right)}^{2}=\frac{2^{3-m-2 i} \pi \Gamma(m-2+i+j)}{(j-i) !\left(j+\frac{m-2}{2}\right)}\|\phi\|_{L^{2}\left(S^{m-2}\right)}^{2} .
$$


Proof. We use the following coordinate:

$$
[-1,1] \times S^{m-2} \rightarrow S^{m-1}, \quad(r, \eta) \mapsto \omega=\left(r, \sqrt{1-r^{2}} \eta\right) .
$$

Then, the standard volume form $d \omega$ on the unit sphere $S^{m-1}$ is given by $\left(1-r^{2}\right)^{\frac{m-3}{2}} d \eta d r$. Therefore,

$$
\left\|I_{i \rightarrow j}^{m}(\phi)\right\|_{L^{2}\left(S^{m-1}\right)}^{2}=\int_{-1}^{1} \int_{S^{m-2}}\left(1-r^{2}\right)^{i}|\phi(\eta)|^{2}\left|\widetilde{C}_{j-i}^{\frac{m-2}{2}+i}(r)\right|^{2}\left(1-r^{2}\right)^{\frac{m-3}{2}} d \eta d r .
$$

Now, apply (7.4.8).

We illustrate the intertwining operator $I_{i j}$ by the two extremal cases, $i=0$ and $i=j$ :

Example 7.5.2. 1) The case $i=0$. Then,

$$
\left(I_{0 \rightarrow j}^{m} \mathbf{1}\right)\left(x_{0}, x\right)=\widetilde{C}_{j}^{\frac{m-2}{2}}\left(x_{0}\right)
$$

is the generator of $O(m-1)$-invariant vectors in $\mathcal{H}^{j}\left(\mathbb{R}^{m}\right)$, where $\mathbf{1}$ is the constant function on $S^{m-1}$.

2) The case $i=j$. Then, we have simply

$$
I_{j \rightarrow j}^{m}(\phi)\left(x_{0}, x\right)=\Gamma(m)|x|^{j} \phi\left(\frac{x}{|x|}\right) .
$$

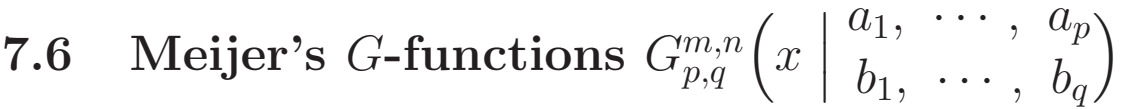

Let $m, n, p$ and $q$ be integers with $0 \leq m \leq q, 0 \leq n \leq p$ and

$$
c^{*}:=m+n-\frac{p+q}{2} \geq 0 .
$$

Suppose further that the complex numbers $a_{1}, \ldots, a_{p}$ and $b_{1}, \ldots, b_{q}$ fulfill the condition:

$$
a_{j}-b_{k} \neq 1,2,3, \ldots \quad(j=1, \ldots n ; k=1, \ldots, m) .
$$


Then, Meijer's G-function of order $(m, n, p, q)$ is defined by the MellinBarnes type integral (see [14, §1.19, §5.3], [55, I, §1], [60, §8.2]): for $x>0$,

$$
\begin{aligned}
G_{p, q}^{m, n}\left(x \mid \begin{array}{lll}
a_{1}, & \cdots, & a_{p} \\
b_{1}, & \cdots, & b_{q}
\end{array}\right) \\
\quad:=\frac{1}{2 \pi \sqrt{-1}} \int_{L} \frac{\prod_{j=1}^{m} \Gamma\left(b_{j}-\lambda\right) \prod_{j=1}^{n} \Gamma\left(1-a_{j}+\lambda\right)}{\prod_{j=m+1}^{q} \Gamma\left(1-b_{j}+\lambda\right) \prod_{j=n+1}^{p} \Gamma\left(a_{j}-\lambda\right)} x^{\lambda} d \lambda,
\end{aligned}
$$

where an empty product is interpreted as 1 .

The contour $L$ starts at the point $\gamma-\sqrt{-1} \infty(\gamma$ is a real number satisfying (7.6.4) below if $c^{*}=0$ ), leaving all the poles of the integrand of the forms

$$
\lambda=b_{j}, b_{j}+1, b_{j}+2, \ldots \quad(1 \leq j \leq m)
$$

to the right, and all the poles of the forms

$$
\lambda=a_{j}-1, a_{j}-2, a_{j}-3, \ldots \quad(1 \leq j \leq n)
$$

to the left of the contour and finishing at the point $\gamma+\sqrt{-1} \infty$.

Here, the condition on the real number $\gamma$ is given by

$$
(q-p) \gamma>\operatorname{Re} \mu
$$

where we set

$$
\mu:=\sum_{j=1}^{q} b_{j}-\sum_{j=1}^{p} a_{j}+\frac{p-q}{2}+1 .
$$

It follows from the asymptotic behavior of the gamma factors (see Lemma 6.1.4) that the integral (7.6.2) converges and is independent of $\gamma$ if one of the following conditions holds:

1) $c^{*}>0,|\arg x|<c^{*} \pi$;

2) $c^{*} \geq 0,|\arg x|=c^{*} \pi,(q-p) \gamma>\operatorname{Re} \mu$.

In particular, the $G$-function extends holomorphically to the complex domain $|\arg x|<c^{*} \pi$ if $c^{*}>0$.

The $G$-function is symmetric in the parameters $a_{1}, \ldots, a_{n}$, likewise in $a_{n+1}, \ldots, a_{p}$, in $b_{1}, \ldots, b_{m}$, and in $b_{m+1}, \ldots, b_{q}$.

Obvious changes of variables in the integral give

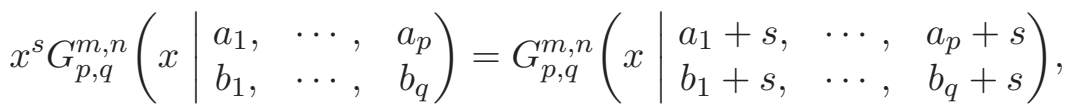

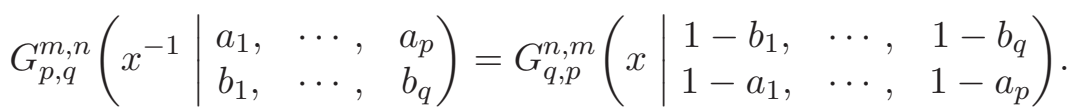


The $G$-function $G_{p, q}^{m, n}\left(x \mid \begin{array}{l}a_{1}, \ldots, a_{p} \\ b_{1}, \ldots, b_{q}\end{array}\right)$ satisfies the differential equation (see [14, $§ 5.4(1)])$ :

$$
\left((-1)^{p-m-n} x \prod_{j=1}^{p}\left(x \frac{d}{d x}-a_{j}+1\right)-\prod_{j=1}^{q}\left(x \frac{d}{d x}-b_{i}\right)\right) u=0 .
$$

If $p<q$, the only singularities of (7.6.5) are $x=0, \infty ; x=0$ is a regular singularity, $x=\infty$ an irregular one. For example, $G_{04}^{20}\left(x \mid b_{1}, b_{2}, b_{3}, b_{4}\right)$ satisfies the fourth order differential equation:

$$
\prod_{j=1}^{4}\left(x \frac{d}{d x}-b_{j}\right) u=0 .
$$

The condition (7.6.1) implies that none of the poles of $\Gamma\left(b_{j}-\lambda\right)(j=$ $1,2, \ldots, m)$ coincides with any of the poles of $\Gamma\left(1-a_{k}+\lambda\right)(k=1, \ldots, n)$. Suppose further that

$$
b_{j}-b_{k} \neq 0, \pm 1, \pm 2, \ldots \quad(1 \leq j<k \leq m) .
$$

Then the integrand (as an ordinary function for $x>0$ ) has simple poles at the points (7.6.3). (We note that as a distribution of $x, x^{\lambda}$ has simple poles at $\lambda=-1,-2,-3, \ldots$, and the analysis involved is more delicate; see Sections 6.1 and 6.2 .) For $p \leq q$, by the residue calculus, we obtain (see [55, I, (7)]):

$$
\begin{aligned}
& G_{p, q}^{m, n}\left(x \mid \begin{array}{lll}
a_{1}, & \cdots, & a_{p} \\
b_{1}, & \cdots, & b_{q}
\end{array}\right) \\
& =\sum_{k=1}^{m} \frac{\prod_{\substack{j=1 \\
j \neq k}}^{m} \Gamma\left(b_{j}-b_{k}\right) \prod_{j=1}^{n} \Gamma\left(1+b_{k}-a_{j}\right)}{\prod_{j=m+1}^{q} \Gamma\left(1+b_{k}-b_{j}\right) \prod_{j=n+1}^{p} \Gamma\left(a_{j}-b_{k}\right)} x^{b_{k}} \\
& \times{ }_{p} F_{q-1}\left(1+b_{k}-a_{1}, \ldots, 1+b_{k}-a_{p} ; 1+b_{k}-b_{1}, \stackrel{\wedge}{\wedge} \cdot 1+b_{k}-b_{q} ;(-1)^{p-m-n} x\right) .
\end{aligned}
$$

Here, ${ }_{p} F_{q}$ denotes the Barnes generalized hypergeometric function:

$$
{ }_{p} F_{q}\left(\alpha_{1}, \ldots, \alpha_{p} ; \beta_{1}, \ldots, \beta_{q} ; x\right)=\sum_{k=0}^{\infty} \frac{x^{k} \prod_{j=1}^{p} \alpha_{j}\left(\alpha_{j}+1\right) \cdots\left(\alpha_{j}+k-1\right)}{k ! \prod_{j=1}^{q} \beta_{j}\left(\beta_{j}+1\right) \cdots\left(\beta_{j}+k-1\right)} .
$$


For example, ${ }_{2} F_{1}\left(\alpha_{1}, \alpha_{2} ; \beta_{1} ; x\right)$ is the Gauss hypergeometric function, and

$$
{ }_{0} F_{1}(\beta ; x)=\sum_{k=0}^{\infty} \frac{x^{k}}{k ! \beta(\beta+1) \cdots(\beta+k-1)} .
$$

Similarly, for $q \leq p$, if $a_{j}-a_{k} \neq 0, \pm 1, \pm 2, \ldots(1 \leq j<k \leq n)$, we have

$$
\begin{aligned}
& G_{p, q}^{m, n}\left(\begin{array}{l|lll}
x & \left.\begin{array}{lll}
a_{1}, & \cdots, & a_{p} \\
b_{1}, & \cdots, & b_{q}
\end{array}\right)
\end{array}\right. \\
& =\sum_{k=1}^{n} \frac{\prod_{\substack{j=1 \\
j \neq k}}^{n} \Gamma\left(a_{k}-a_{j}\right) \prod_{j=1}^{n} \Gamma\left(b_{j}-a_{k}+1\right)}{\prod_{j=n+1}^{p} \Gamma\left(a_{j}-a_{k}+1\right) \prod_{j=m+1}^{q} \Gamma\left(a_{k}-b_{j}\right)} x^{a_{k}-1} \\
& \times_{q} F_{p-1}\left(1+b_{1}-a_{k}, \ldots, 1+b_{q}-a_{k} ; 1+a_{1}-a_{k}, \stackrel{k}{\wedge}, 1+a_{p}-a_{k} ;(-1)^{q-m-n} x^{-1}\right) .
\end{aligned}
$$

For $p \leq q$, it follows from (7.6.7) that

$$
G_{p, q}^{m, n}\left(x \mid \begin{array}{lll}
a_{1}, & \cdots, & a_{p} \\
b_{1}, & \cdots, & b_{q}
\end{array}\right)=O\left(|x|^{\min \left(\operatorname{Re} b_{1}, \ldots, \operatorname{Re} b_{m}\right)}\right)
$$

as $x \rightarrow 0$ (see also [14, I, $\S 5.4 .1$ (8)], but there is a typographical error: $\max \operatorname{Re} b_{h}$ loc. cit. should be $\left.\min \operatorname{Re} b_{h}\right)$. On the other hand, the asymptotic expansion of $G_{p, q}^{m, n}(x)(p \leq q)$ for large $x>0$ that we need in this book is the following case:

Fact 7.6.1 ([55, VII, Theorem 17]). Let $m, p$ and $q$ be integers satisfying

$$
0 \leq p \leq q-2 \text { and } p+1 \leq m \leq q-1 .
$$

Then the G-function $G_{p, q}^{m, 0}(x)$ possesses the following asymptotic expansion for large $x>0$ :

$$
G_{p, q}^{m, 0}(x) \sim A_{q}^{m, 0} H_{p, q}\left(x e^{(q-m) \pi \sqrt{-1}}\right)+\bar{A}_{q}^{m, 0} H_{p, q}\left(x e^{(m-q) \pi \sqrt{-1}}\right) .
$$

Here, $H_{p, q}(z)$ is a function that possesses the following expansion (see [55, I, (25)]):

$$
H_{p, q}(z)=\exp \left((p-q) z^{\frac{1}{q-p}}\right) z^{\theta}\left(\frac{(2 \pi)^{\frac{q-p-1}{2}}}{\sqrt{q-p}}+\frac{M_{1}}{z^{\frac{1}{q-p}}}+\frac{M_{2}}{z^{\frac{2}{q-p}}}+\cdots\right),
$$


where $M_{1}, M_{2}, \ldots$ are constants, and $\theta$ is given by

$$
\theta:=\frac{1}{q-p}\left(\frac{p-q+1}{2}+\sum_{j=1}^{q} b_{j}-\sum_{j=1}^{p} a_{j}\right), \quad[55, \mathbf{I},(23)] .
$$

The coefficients $A_{q}^{m, 0}$ and $\bar{A}_{q}^{m, 0}$ are given by

$$
\begin{array}{rlr}
A_{q}^{m, 0} & :=(-2 \pi \sqrt{-1})^{m-q} e^{-\left(b_{m+1}+\cdots+b_{q}\right) \pi \sqrt{-1}}, & \text { [55, II, (45)], } \\
\bar{A}_{q}^{m, 0} & :=(2 \pi \sqrt{-1})^{m-q} e^{\left(b_{m+1}+\cdots+b_{q}\right) \pi \sqrt{-1}}, & \text { [55, II, (46)]. }
\end{array}
$$

Example 7.6.2. For $(m, n, p, q)=(2,0,1,3), c^{*}=0$. We take $\gamma$ such that

$$
\gamma>\frac{1}{2} \operatorname{Re}\left(b_{1}+b_{2}+b_{3}-a_{1}\right)
$$

Then, we have an integral expression:

$$
G_{13}^{20}\left(\begin{array}{l|c}
x & \begin{array}{c}
a_{1} \\
b_{1}, b_{2}, b_{3}
\end{array}
\end{array}\right)=\frac{1}{2 \pi \sqrt{-1}} \int_{L} \frac{\Gamma\left(b_{1}-\lambda\right) \Gamma\left(b_{2}-\lambda\right)}{\Gamma\left(1-b_{3}+\lambda\right) \Gamma\left(a_{1}-\lambda\right)} x^{\lambda} d \lambda,
$$

where the integral path $L$ starts from $\gamma-\sqrt{-1} \infty$, leaves $b_{1}, b_{2}$ to the right and ends at $\gamma+\sqrt{-1} \infty$ (see Figure 7.6.1).

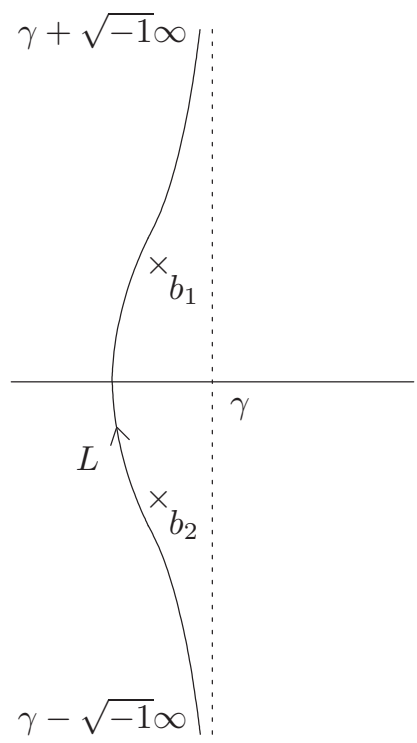

Figure 7.6.1 
Example 7.6.3. If $p=0$, the $G$-function is denoted by $G_{0, q}^{m, 0}\left(x \mid b_{1}, \ldots, b_{q}\right)$. The G-function that we use most frequently in this book is of type $G_{04}^{20}$. Again, we have $c^{*}=0$. Then, we have an integral expression:

$$
G_{04}^{20}\left(x \mid b_{1}, b_{2}, b_{3}, b_{4}\right)=\frac{1}{2 \pi \sqrt{-1}} \int_{L} \frac{\Gamma\left(b_{1}-\lambda\right) \Gamma\left(b_{2}-\lambda\right)}{\Gamma\left(1-b_{3}+\lambda\right) \Gamma\left(1-b_{4}+\lambda\right)} x^{\lambda} d \lambda,
$$

where $L$ starts from $\gamma-\sqrt{-1} \infty$, leaves $b_{1}, b_{2}$ to the right, and ends at $\gamma+\sqrt{-1} \infty$ (see Figure 7.6.1) for $\gamma \in \mathbb{R}$ such that

$$
\gamma>\frac{1}{4}\left(\operatorname{Re}\left(b_{1}+b_{2}+b_{3}+b_{4}\right)-1\right) .
$$

In Section 4.5, we need the following lemma on the asymptotic behavior:

Lemma 7.6.4. The asymptotic behavior of the $G$-functions $G_{04}^{20}\left(x \mid b_{1}, b_{2}, 1-\right.$ $\left.\gamma-b_{1}, 1-\gamma-b_{2}\right)$ are given as follows:

1) As $x$ tends to $0, G_{04}^{20}\left(x \mid b_{1}, b_{2}, 1-\gamma-b_{1}, 1-\gamma-b_{2}\right)=O\left(x^{\min \left(b_{1}, b_{2}\right)}\right)$.

2) As $x$ tends to $\infty$,

$$
\begin{aligned}
& G_{04}^{20}\left(x \mid b_{1}, b_{2}, 1-\gamma-b_{1}, 1-\gamma-b_{2}\right) \\
= & -\frac{1}{\sqrt{2 \pi}} x^{\frac{1-4 \gamma}{8}} \cos \left(4 x^{\frac{1}{4}}-\left(\gamma+b_{1}+b_{2}+\frac{1}{4}\right) \pi\right)\left(1+P_{1} x^{-\frac{1}{2}}+P_{2} x^{-1}+\cdots\right) \\
& +x^{\frac{1-4 \gamma}{8}} \sin \left(4 x^{\frac{1}{4}}-\left(\gamma+b_{1}+b_{2}+\frac{1}{4}\right) \pi\right)\left(Q_{1} x^{-\frac{1}{4}}+Q_{2} x^{-\frac{3}{4}}+\cdots\right) .
\end{aligned}
$$

Here, $P_{1}, \cdots, Q_{1}, \cdots$ are the constants independent of $x$.

Proof. 1) This estimate is a special case of (7.6.10).

2) We apply Fact 7.6.1 to the case

$$
(m, p, q)=(2,0,4), \quad\left(b_{1}, b_{2}, b_{3}, b_{4}\right)=\left(b_{1}, b_{2}, 1-\gamma-b_{1}, 1-\gamma-b_{2}\right) .
$$

Then, the coefficients $A_{4}^{20}, \bar{A}_{4}^{20}$ and the constant $\theta$ amount to

$$
\begin{aligned}
& A_{4}^{20}=-\frac{1}{4 \pi^{2}} e^{\left(2 \gamma-2+b_{1}+b_{2}\right) \pi \sqrt{-1}}, \quad \bar{A}_{4}^{20}=-\frac{1}{4 \pi^{2}} e^{-\left(2 \gamma-2+b_{1}+b_{2}\right) \pi \sqrt{-1}}, \\
& \theta=\frac{1-4 \gamma}{8} .
\end{aligned}
$$

The expansion of $H_{0,4}\left(x e^{ \pm 2 \pi \sqrt{-1}}\right)$ is given by

$$
H_{0,4}\left(x e^{ \pm 2 \pi \sqrt{-1}}\right)=e^{\mp\left(4 x^{\frac{1}{4}}-\frac{1-4 \gamma}{4} \pi\right) \sqrt{-1}} x^{\frac{1-4 \gamma}{8}}\left(\frac{(2 \pi)^{\frac{3}{2}}}{2} \pm \frac{M_{1}}{\sqrt{-1} x^{\frac{1}{4}}}+\cdots\right) .
$$


Hence, $G_{04}^{20}(x)$ has the following asymptotic expansion:

$$
\begin{aligned}
& -\frac{1}{4 \pi^{2}} e^{-\left(4 x^{\frac{1}{4}}-\pi\left(\gamma+b_{1}+b_{2}-\frac{7}{4}\right)\right) \sqrt{-1}} x^{\frac{1-4 \gamma}{8}}\left(\frac{(2 \pi)^{\frac{3}{2}}}{2}+\frac{M_{1}}{\sqrt{-1} x^{\frac{1}{4}}}+\cdots\right) \\
& -\frac{1}{4 \pi^{2}} e^{\left(4 x^{\frac{1}{4}}-\pi\left(\gamma+b_{1}+b_{2}-\frac{7}{4}\right)\right) \sqrt{-1}} x^{\frac{1-4 \gamma}{8}}\left(\frac{(2 \pi)^{\frac{3}{2}}}{2}-\frac{M_{1}}{\sqrt{-1} x^{\frac{1}{4}}}+\cdots\right),
\end{aligned}
$$

which is expressed as the right-hand side of (7.6.11) by virtue of the formulas $e^{c \pi \sqrt{-1}}+e^{-c \pi \sqrt{-1}}=2 \cos (c \pi)$ and $e^{c \pi \sqrt{-1}}-e^{-c \pi \sqrt{-1}}=2 \sqrt{-1} \sin (c \pi)$.

Finally, we list the reduction formulas of $G$-functions that are used in this book:

$$
\begin{array}{rlrl}
G_{02}^{10}(x \mid a, b) & =x^{\frac{1}{2}(a+b)} J_{a-b}\left(2 x^{\frac{1}{2}}\right) & & {[14, \S 5.6(3)],} \\
G_{02}^{20}(x \mid a, b) & =2 x^{\frac{1}{2}(a+b)} K_{a-b}\left(2 x^{\frac{1}{2}}\right) & {[14, \S 5.6(4)],} \\
G_{04}^{20}\left(x \mid a, a+\frac{1}{2}, b, b+\frac{1}{2}\right) & =x^{\frac{1}{2}(a+b)} J_{2(a-b)}\left(4 x^{\frac{1}{4}}\right) & {[14, \S 5.6(11)],} \\
G_{13}^{20}\left(x \mid \begin{array}{c}
a-\frac{1}{2} \\
a, b, a-\frac{1}{2}
\end{array}\right) & =x^{\frac{1}{2}(a+b)} Y_{b-a}\left(2 x^{\frac{1}{2}}\right) & {[14, \S 5.6(23)] .}
\end{array}
$$

\subsection{Appell's hypergeometric functions $F_{1}, F_{2}, F_{3}, F_{4}$}

Appell's hypergeometric functions (in two variables) $F_{1}, F_{2}, F_{3}, F_{4}$ are defined by the following double power series:

$$
\begin{aligned}
F_{1}\left(\alpha, \beta, \beta^{\prime}, \gamma ; x, y\right) & :=\sum_{m, n=0}^{\infty} \frac{(\alpha)_{m+n}(\beta)_{m}\left(\beta^{\prime}\right)_{n}}{(\gamma)_{m+n} m ! n !} x^{m} y^{n}, \\
F_{2}\left(\alpha, \beta, \beta^{\prime}, \gamma, \gamma^{\prime} ; x, y\right) & :=\sum_{m, n=0}^{\infty} \frac{(\alpha)_{m+n}(\beta)_{m}\left(\beta^{\prime}\right)_{n}}{(\gamma)_{m}\left(\gamma^{\prime}\right)_{n} m ! n !} x^{m} y^{n}, \\
F_{3}\left(\alpha, \alpha^{\prime}, \beta, \beta^{\prime}, \gamma ; x, y\right) & :=\sum_{m, n=0}^{\infty} \frac{(\alpha)_{m}\left(\alpha^{\prime}\right)_{n}(\beta)_{m}\left(\beta^{\prime}\right)_{n}}{(\gamma)_{m+n} m ! n !} x^{m} y^{n}, \\
F_{4}\left(\alpha, \beta, \gamma, \gamma^{\prime} ; x, y\right) & :=\sum_{m, n=0}^{\infty} \frac{(\alpha)_{m+n}(\beta)_{m+n}}{(\gamma)_{m}\left(\gamma^{\prime}\right)_{n} m ! n !} x^{m} y^{n} .
\end{aligned}
$$

Ap1 Reduction from $F_{3}$ to $F_{1}([14, \S 5.11,(11)])$ :

$$
F_{3}\left(\alpha, \alpha^{\prime}, \beta, \beta^{\prime}, \alpha+\alpha^{\prime} ; x, y\right)=(1-y)^{-\beta^{\prime}} F_{1}\left(\alpha, \beta, \beta^{\prime}, \alpha+\alpha^{\prime} ; x, \frac{y}{y-1}\right) \text {. }
$$


Ap2 Reduction from $F_{3}$ to ${ }_{2} F_{1}([14, \S 5.10,(4)])$ :

$$
F_{3}(\alpha, \gamma-\alpha, \beta, \gamma-\beta ; \gamma ; x, y)=(1-y)^{\alpha+\beta-\gamma}{ }_{2} F_{1}(\alpha, \beta, \gamma ; x+y-x y) .
$$

Ap3 Reduction formula of $F_{4}([14, \S 5.10,(8)])$ :

$$
\begin{aligned}
& F_{4}\left(\alpha, \beta ; 1+\alpha-\beta, \beta ; \frac{-x}{(1-x)(1-y)}, \frac{-y}{(1-x)(1-y)}\right) \\
= & (1-y)^{\alpha}{ }_{2} F_{1}\left(\alpha, \beta ; 1+\alpha-\beta ; \frac{-x(1-y)}{1-x}\right) .
\end{aligned}
$$

Ap4 Single integral of Euler's type for $F_{1}([14, \S 5.8 .2,(5)])$ : For $\operatorname{Re} \alpha>0$ and $\operatorname{Re}(\gamma-\alpha)>0$

$$
\begin{aligned}
& F_{1}\left(\alpha, \beta, \beta^{\prime}, \gamma ; x, y\right)= \\
& \quad \frac{\Gamma(\gamma)}{\Gamma(\alpha) \Gamma(\gamma-\alpha)} \int_{0}^{1} u^{\alpha-1}(1-u)^{\gamma-\alpha-1}(1-u x)^{-\beta}(1-u y)^{-\beta^{\prime}} d u
\end{aligned}
$$

Ap5 Double integral of Euler's type for $F_{3}([14, \S 5.8 .1,(3)])$ :

$$
\begin{aligned}
& F_{3}\left(\alpha, \alpha^{\prime}, \beta, \beta^{\prime}, \gamma ; x, y\right)=\frac{\Gamma(\gamma)}{\Gamma(\beta) \Gamma\left(\beta^{\prime}\right) \Gamma\left(\gamma-\beta-\beta^{\prime}\right)} \\
& \times \iint_{D} u^{\beta-1} v^{\beta^{\prime}-1}(1-u-v)^{\gamma-\beta-\beta^{\prime}-1}(1-u x)^{-\alpha}(1-v y)^{-\alpha^{\prime}} d u d v \\
& \operatorname{Re} \beta>0, \operatorname{Re} \beta^{\prime}>0, \operatorname{Re}\left(\gamma-\beta-\beta^{\prime}\right)>0,
\end{aligned}
$$

where $D:=\left\{(u, v) \in \mathbb{R}^{2}: u \geq 0, v \geq 0, u+v \leq 1\right\}$.

\subsection{Hankel transform with trigonometric parame- ters}

This section presents an integral formula (7.8.1) on the Hankel transform with two trigonometric parameters. In the conformal model [47] (i.e. the solution space to the Yamabe equation) of the minimal representation, $K$ finite vectors can be explicitly expressed in terms of spherical harmonics (e.g. Gegenbauer's polynomials). On the other hand, in the $L^{2}$-model (the Schrödinger model) which is obtained by the Euclidean Fourier transform 
of the conformal model (or the $N$-picture in a terminology of representation theory), it is not easy to find explicit $K$-finite vectors. The formula (7.8.1) bridges these two models and gives an explicit formula of $K$-finite vectors in the Schrödinger model (see the proof of Lemma 3.4.4).

Since we have not found this formula in the literature, we give a proof here for the sake of completeness. The method here is a generalization of the argument in [49, $§ 5.6,5.7]$.

Lemma 7.8.1. The following integral formula on the Hankel transform holds:

$$
\begin{aligned}
& \int_{0}^{\infty} t^{\mu+1} J_{\mu}\left(\frac{t \sin \theta}{\cos \theta+\cos \phi}\right) J_{\nu}\left(\frac{t \sin \phi}{\cos \theta+\cos \phi}\right) K_{\nu}(t) d t \\
= & \frac{2^{\nu-1}}{\sqrt{\pi}} \Gamma(\mu-\nu+1)(\cos \theta+\cos \phi) \sin ^{\mu} \theta \sin ^{\nu} \phi \widetilde{C}_{\mu-\nu}^{\nu+\frac{1}{2}}(\cos \phi) .
\end{aligned}
$$

Proof. By Baily's formula (7.2.12) of the Hankel transform, the left-hand side of (7.8.1) equals

$$
\begin{aligned}
& \frac{\Gamma(\mu+\nu+1)}{\Gamma(\nu+1)} \frac{2^{\mu} \sin ^{\mu} \theta \sin ^{\nu} \phi}{(\cos \theta+\cos \phi)^{\mu+\nu}} \\
& \times F_{4}\left(\mu+1, \mu+\nu+1 ; \mu+1, \nu+1 ;-\left(\frac{\sin \theta}{\cos \theta+\cos \phi}\right)^{2},-\left(\frac{\sin \phi}{\cos \theta+\cos \phi}\right)^{2}\right) .
\end{aligned}
$$

Here $F_{4}$ denotes Appell's hypergeometric function (see (7.7.4)). Thus, the proof of Lemma 7.8 .1 will be completed if we show the following:

Claim 7.8.2. We have

$$
\begin{gathered}
F_{4}\left(\mu+1, \mu+\nu+1 ; \mu+1, \nu+1 ;-\left(\frac{\sin \theta}{\cos \theta+\cos \phi}\right)^{2},-\left(\frac{\sin \phi}{\cos \theta+\cos \phi}\right)^{2}\right) \\
=\frac{(\cos \theta+\cos \phi)^{\mu+\nu+1}}{2^{\mu-\nu+1} \sqrt{\pi}} \frac{\Gamma(\mu-\nu+1) \Gamma(\nu+1)}{\Gamma(\mu+\nu+1)} \widetilde{C}_{\mu-\nu}^{\nu+\frac{1}{2}}(\cos \phi) .
\end{gathered}
$$

Claim 7.8 .2 is essentially a restatement of [49, Lemma 5.7]. For the convenience of the reader, we include its proof here.

Proof of Claim 7.8.2. We recall a quadratic transformation for hypergeometric functions (see [14, $\S 2.11(32)]$ ):

$$
{ }_{2} F_{1}(\alpha, \beta ; 1+\alpha-\beta ; z)=(1-z)^{-\alpha}{ }_{2} F_{1}\left(\frac{\alpha}{2}, \frac{\alpha+1-2 \beta}{2} ; 1+\alpha-\beta ; \frac{-4 z}{(1-z)^{2}}\right) .
$$


Combining the reduction formula (7.7.7) with (7.8.4), and using the symmetry of $a$ and $b ;(c, x)$ and $(d, y)$ in $F_{4}(a, b ; c, d ; x, y)$, we have

$$
\begin{aligned}
& F_{4}\left(\alpha, \beta ; \alpha, 1-\alpha+\beta ; \frac{-x}{(1-x)(1-y)}, \frac{-y}{(1-x)(1-y)}\right) \\
& =\left(\frac{(1-x)(1-y)}{1-x y}\right){ }_{2}^{\beta} F_{1}\left(\frac{\beta}{2}, \frac{1-2 \alpha+\beta}{2} ; 1-\alpha+\beta ; \frac{4 y(1-x)(1-y)}{(1-x y)^{2}}\right) .
\end{aligned}
$$

Consider the change of variables from $(x, y)$ to $(\theta, \phi)$ by the following identities:

$$
\frac{x}{(1-x)(1-y)}=\left(\frac{\sin \theta}{\cos \theta+\cos \phi}\right)^{2}, \quad \frac{y}{(1-x)(1-y)}=\left(\frac{\sin \phi}{\cos \theta+\cos \phi}\right)^{2}
$$

such that $(x, y)=(0,0)$ corresponds to $(\theta, \phi)=(0,0)$. Then, a simple computation shows

$$
\frac{1-x y}{(1-x)(1-y)}=\frac{2}{\cos \theta+\cos \phi}, \quad \frac{4 y(1-x)(1-y)}{(1-x y)^{2}}=\sin ^{2} \phi
$$

Now, we set

$$
\alpha=\mu+1, \quad \beta=\mu+\nu+1,
$$

in (7.8.5). Then, the left-hand side of (7.8.3) amounts to

$$
\left(\frac{2}{\cos \theta+\cos \phi}\right)_{2}^{-\mu-\nu-1} F_{1}\left(\frac{\mu+\nu+1}{2},-\frac{\mu-\nu}{2} ; \nu+1 ; \sin ^{2} \phi\right) .
$$

By using (7.4.6), (7.8.6) is expressed as

$$
\left(\frac{\cos \theta+\cos \phi}{2}\right)^{\mu+\nu+1} \frac{\Gamma(\mu-\nu+1) \Gamma(2 \nu+1)}{\Gamma(\mu+\nu+1) \Gamma\left(\nu+\frac{1}{2}\right)} \widetilde{C}_{\mu-\nu}^{\nu+\frac{1}{2}}(\cos \phi) .
$$

By using Gauss's duplication formula (7.4.3), we get Claim.

\subsection{Fractional integral of two variables}

In Section 5.3, we find explicit eigenvalues of intertwining operators on $L^{2}\left(S^{p-2} \times S^{q-2}\right)$ based on the Funk-Hecke formula (see Example 5.3.2). The following lemma is the substantial part of computation there. 
Lemma 7.9.1. For $\operatorname{Re} \mu, \operatorname{Re} \nu>-\frac{1}{2}, \operatorname{Re} \lambda>-1$ and $l, k \in \mathbb{N}$, we have the following formula for the fractional integral:

$$
\begin{aligned}
& \int_{-1}^{1} \int_{-1}^{1} \frac{(x+y)_{ \pm}^{\lambda}}{\Gamma(\lambda+1)} \widetilde{C}_{l}^{\mu}(x) \widetilde{C}_{k}^{\nu}(y)\left(1-x^{2}\right)^{\mu-\frac{1}{2}}\left(1-y^{2}\right)^{\nu-\frac{1}{2}} d x d y \\
& =\frac{b 2^{1-\lambda} \Gamma(\lambda+\mu+\nu+1)}{\Gamma\left(\frac{\lambda+2 \mu+2 \nu+l+k+2}{2}\right) \Gamma\left(\frac{\lambda+2 \mu+l-k+2}{2}\right) \Gamma\left(\frac{\lambda+2 \nu-l+k+2}{2}\right) \Gamma\left(\frac{\lambda-l-k+2}{2}\right)},
\end{aligned}
$$

where

$$
b:=\frac{( \pm 1)^{l+k} \pi^{2}}{2^{2 \mu+2 \nu}} \frac{\Gamma(2 \mu+l) \Gamma(2 \nu+k)}{l ! k !}
$$

is a constant independent of $\lambda$.

Proof. The left-hand side of (7.9.1) amounts to

$$
\begin{aligned}
& \int_{-1}^{1}\left(\int_{-x}^{1} \frac{(x+y)^{\lambda}}{\Gamma(\lambda+1)} \widetilde{C}_{k}^{\nu}(y)\left(1-y^{2}\right)^{\nu-\frac{1}{2}} d y\right) \widetilde{C}_{l}^{\mu}(x)\left(1-x^{2}\right)^{\mu-\frac{1}{2}} d x \\
= & \frac{\sqrt{\pi} \Gamma(2 \nu+k)}{2^{\nu-\frac{1}{2}} k !} \int_{-1}^{1}\left(\left(1-x^{2}\right)^{\frac{\lambda}{2}+\frac{\nu}{2}+\frac{1}{4}} P_{\nu+k-\frac{1}{2}}^{-\lambda-\nu-\frac{1}{2}}(-x)\right) \widetilde{C}_{l}^{\mu}(x)\left(1-x^{2}\right)^{\mu-\frac{1}{2}} d x \\
= & \frac{2^{-\nu-2 \mu-l+\frac{3}{2}} \pi}{\Gamma\left(\mu+l+\frac{1}{2}\right)} \frac{\Gamma(2 \nu+k) \Gamma(2 \mu+l)}{k ! l !} \int_{-1}^{1}\left(1-x^{2}\right)^{\frac{\lambda}{2}+\mu+\frac{\nu}{2}-\frac{l}{2}-\frac{1}{4}} P_{\nu+k-\frac{1}{2}}^{-\lambda-\nu+l-\frac{1}{2}}(-x) d x \\
= & \frac{2^{1-\lambda-2 \mu-2 \nu} \pi \Gamma(2 \mu+l) \Gamma(2 \nu+k) \Gamma(\lambda+\mu+\nu+1)}{l ! k ! \Gamma\left(\frac{\lambda+2 \mu+2 \nu+l+k+2}{2}\right) \Gamma\left(\frac{\lambda+2 \mu+l-k+2}{2}\right) \Gamma\left(\frac{\lambda+2 \nu-l+k+2}{2}\right) \Gamma\left(\frac{\lambda-l-k+2}{2}\right)} .
\end{aligned}
$$

Hence, the right-hand side of (7.9.1) follows. Some remarks on each equality are given in turn:

First equality follows from Ge 4 in Appendix 7.4 .

Second equality. First, we made use of the integral by parts because we have (see (7.4.1))

$$
\widetilde{C}_{l}^{\mu}(x)\left(1-x^{2}\right)^{\mu-\frac{1}{2}}=\frac{(-1)^{l}}{2^{2 \mu+l-1} l !} \frac{\Gamma(2 \mu+l) \sqrt{\pi}}{\Gamma\left(\mu+l+\frac{1}{2}\right)} \frac{d^{l}}{d x^{l}}\left(\left(1-x^{2}\right)^{\mu+l-\frac{1}{2}}\right) .
$$

Then, we applied the functional relation

$$
\frac{d^{l}}{d x^{l}}\left(\left(1-x^{2}\right)^{\frac{\lambda}{2}+\frac{\nu}{2}+\frac{1}{4}} P_{\nu+k-\frac{1}{2}}^{-\lambda-\nu-\frac{1}{2}}(-x)\right)=\left(1-x^{2}\right)^{\frac{\lambda}{2}+\frac{\nu}{2}-\frac{l}{2}+\frac{1}{4}} P_{\nu+k-\frac{1}{2}}^{-\lambda-\nu+l-\frac{1}{2}}(-x),
$$

which is obtained by iterating (7.3.2).

Third equality. We applied the integral formula (7.3.4) after changing the variable $x \mapsto-x$.

Therefore, the proof of Lemma 7.9.1 is completed. 


\section{Bibliography}

[1] G. E. Andrews, R. Askey, and R. Roy, Special functions, Encyclopedia of Mathematics and its Applications, vol. 71, Cambridge University Press, Cambridge, 2000.

[2] P. Appell and J. Kampé de Fériet, Fonctions hypergéométriques et hypersphériques: Polynomes d'Hermite, Gauthiers-Villars, Paris, 1926.

[3] W. N. Bailey, Some infinite integrals involving Bessel functions, Proc. London Math. Soc. (2) 40 (1935-36), 37-48.

[4] D. Barbasch and D. A. Vogan, Jr., The local structure of characters, J. Funct. Anal. 37 (1980), 27-55.

[5] I. N. Bernštel̆n and S. I. Gel'fand, Meromorphy of the function $P^{\lambda}$, Funkcional. Anal. i Priložen. 3 (1969), 84-85.

[6] B. Binegar and R. Zierau, Unitarization of a singular representation of $\mathrm{SO}(p, q)$, Comm. Math. Phys. 138 (1991), 245-258.

[7] R. Brylinski and B. Kostant, Differential operators on conical Lagrangian manifolds, Lie theory and geometry, Progr. Math., vol. 123, Birkhäuser Boston, Boston, MA, 1994, pp. 65-96.

[8] J.-L. Clerc, Laplace transform and unitary highest weight modules, J. Lie Theory 5 (1995), 225-240.

[9] L. Debnath and D. Bhatta, Integral transforms and their applications, second ed., Chapman \& Hall/CRC, Boca Raton, FL, 2007.

[10] H. Ding, K. I. Gross, R. A. Kunze, and D. St. P. Richards, Bessel functions on boundary orbits and singular holomorphic representations, The mathematical legacy of Harish-Chandra (Baltimore, MD, 1998), Proc. Sympos. Pure Math., vol. 68, Amer. Math. Soc., Providence, RI, 2000, pp. 223-254. 
[11] M. Duflo, Théorie de Mackey pour les groupes de Lie algébriques, Acta Math. 149 (1982), 153-213.

[12] A. Dvorsky, Tensor square of the minimal representation of $\mathrm{O}(p, q)$, Canad. Math. Bull. 50 (2007), 48-55.

[13] A. Dvorsky and S. Sahi, Explicit Hilbert spaces for certain unipotent representations. II, Invent. Math. 138 (1999), 203-224.

[14] A. Erdélyi, W. Magnus, F. Oberhettinger, and F. G. Tricomi, Higher transcendental functions. Vols. I, II, McGraw-Hill Book Company, Inc., New York-Toronto-London, 1953.

[15] A. Erdélyi, W. Magnus, F. Oberhettinger, and F. G. Tricomi, Tables of integral transforms. Vol. II, McGraw-Hill Book Company, Inc., New York-Toronto-London, 1954.

[16] J. Faraut and A. Korányi, Analysis on symmetric cones, Oxford Mathematical Monographs, The Clarendon Press, Oxford University Press, New York, 1994.

[17] Y. Flicker, D. Kazhdan, and G. Savin, Explicit realization of a metaplectic representation, J. Analyse Math. 55 (1990), 17-39.

[18] G. B. Folland, Harmonic analysis in phase space, Annals of Mathematics Studies, vol. 122, Princeton University Press, Princeton, NJ, 1989.

[19] C. Fox, The $G$ and $H$ functions as symmetrical Fourier kernels, Trans. Amer. Math. Soc. 98 (1961), 395-429.

[20] W.-T. Gan and G. Savin, Uniqueness of Joseph ideal, Math. Res. Lett. 11 (2004), 589-597.

[21] W.-T. Gan and G. Savin, On minimal representations definitions and properties, Represent. Theory 9 (2005), 46-93.

[22] S. Gelbart, Examples of dual reductive pairs, Automorphic forms, representations and $L$-functions (Proc. Sympos. Pure Math., Oregon State Univ., Corvallis, Ore., 1977), Part 1, Proc. Sympos. Pure Math., XXXIII, Amer. Math. Soc., Providence, R.I., 1979, pp. 287-296.

[23] I. M. Gel'fand and S. G. Gindikin, Complex manifolds whose spanning trees are real semisimple Lie groups, and analytic discrete series of representations, Funkcional. Anal. i Priložen. 11 (1977), 19-27, 96. 
[24] I. M. Gel'fand and G. E. Shilov, Generalized functions. Vols. 1, 2, Academic Press, New York, 1964.

[25] I. M. Gel'fand, M. I. Graev, and N. Ya. Vilenkin, Generalized functions. Vol. 5, Academic Press, New York, 1966.

[26] I. S. Gradshteyn and I. M. Ryzhik, Table of integrals, series, and products, Academic Press, New York, 1965.

[27] B. H. Gross and N. R. Wallach, A distinguished family of unitary representations for the exceptional groups of real rank $=4$, Lie theory and geometry, Progr. Math., vol. 123, Birkhäuser Boston, Boston, MA, 1994, pp. 289-304.

[28] V. Guillemin and S. Sternberg, Variations on a theme by Kepler, American Mathematical Society Colloquium Publications, vol. 42, American Mathematical Society, Providence, RI, 1990.

[29] H. Hankel, Die Fourier'schen Reihen und Integrale für Cylinderfunctionen, Math. Ann. 8 (1875), 471-494.

[30] G. H. Hardy, Summation of a series of polynomials of Laguerre, J. London Math. Soc. 7 (1932), 138-139, 192.

[31] S. Helgason, Groups and geometric analysis, Pure and Applied Mathematics, vol. 113, Academic Press Inc., Orlando, FL, 1984.

[32] J. Hilgert, K.-H. Neeb, and B. Ørsted, Conal Heisenberg algebras and associated Hilbert spaces, J. Reine Angew. Math. 474 (1996), 67-112.

[33] L. Hörmander, The analysis of linear partial differential operators. I, Springer-Verlag, Berlin, 1984.

[34] R. Howe, Wave front sets of representations of Lie groups, Automorphic forms, representation theory and arithmetic (Bombay, 1979), Tata Inst. Fund. Res. Studies in Math., vol. 10, Tata Inst. Fundamental Res., Bombay, 1981, pp. 117-140.

[35] R. Howe, The oscillator semigroup, The mathematical heritage of Hermann Weyl (Durham, NC, 1987), Proc. Sympos. Pure Math., vol. 48, Amer. Math. Soc., Providence, RI, 1988, pp. 61-132.

[36] R. E. Howe and E.-C. Tan, Homogeneous functions on light cones: the infinitesimal structure of some degenerate principal series representations, Bull. Amer. Math. Soc. (N.S.) 28 (1993), 1-74. 
[37] A. Joseph, The minimal orbit in a simple Lie algebra and its associated maximal ideal, Ann. Sci. École Norm. Sup. (4) 9 (1976), 1-29.

[38] M. Kashiwara and M. Vergne, On the Segal-Shale-Weil representations and harmonic polynomials, Invent. Math. 44 (1978), 1-47.

[39] D. Kazhdan, The minimal representation of $D_{4}$, Operator algebras, unitary representations, enveloping algebras, and invariant theory (Paris, 1989), Progr. Math., vol. 92, Birkhäuser Boston, Boston, MA, 1990, pp. $125-158$.

[40] D. Kazhdan and G. Savin, The smallest representation of simply laced groups, Festschrift in honor of I. I. Piatetski-Shapiro on the occasion of his sixtieth birthday, Part I (Ramat Aviv, 1989), Israel Math. Conf. Proc., vol. 2, Weizmann, Jerusalem, 1990, pp. 209-223.

[41] A. W. Knapp, Representation theory of semisimple groups: an overview based on examples, Princeton University Press, Princeton, NJ, 1986.

[42] S. Kobayashi, Transformation groups in differential geometry, Classics in Mathematics, Springer-Verlag, Berlin, 1995, Reprint of the 1972 edition.

[43] T. Kobayashi, Conformal geometry and global solutions to the Yamabe equations on classical pseudo-Riemannian manifolds, Proceedings of the 22nd Winter School "Geometry and Physics" (Srní, 2002), Rend. Circ. Mat. Palermo (2) Suppl. 71, 2003, pp. 15-40.

[44] T. Kobayashi and G. Mano, Integral formulas for the minimal representation of $\mathrm{O}(p, 2)$, Acta Appl. Math. 86 (2005), 103-113.

[45] T. Kobayashi and G. Mano, Integral formula of the unitary inversion operator for the minimal representation of $\mathrm{O}(p, q)$, Proc. Japan Acad. Ser. A Math. Sci. 83 (2007), 27-31.

[46] T. Kobayashi and G. Mano, The inversion formula and holomorphic extension of the minimal representation of the conformal group, Harmonic Analysis, Group Representations, Automorphic Forms and Invariant Theory: In honor of Roger E. Howe (J.-S. Li, E.-C. Tan, N. Wallach, and C.-B. Zhu, eds.), Singapore University Press and World Scientific Publishing, 2007, pp. 159-223 (cf. math.RT/0607007). 
[47] T. Kobayashi and B. Ørsted, Analysis on the minimal representation of $\mathrm{O}(p, q)$. I. Realization via conformal geometry, Adv. Math. 180 (2003), 486-512.

[48] T. Kobayashi and B. Ørsted, Analysis on the minimal representation of $\mathrm{O}(p, q)$. II. Branching laws, Adv. Math. 180 (2003), 513-550.

[49] T. Kobayashi and B. Ørsted, Analysis on the minimal representation of $\mathrm{O}(p, q)$. III. Ultrahyperbolic equations on $\mathbb{R}^{p-1, q-1}$, Adv. Math. 180 (2003), 551-595.

[50] B. Kostant, The vanishing of scalar curvature and the minimal representation of $\mathrm{SO}(4,4)$, Operator algebras, unitary representations, enveloping algebras, and invariant theory (Paris, 1989) (A. Connes, M. Duflo, A. Joseph, and R. Rentschler, eds.), Progr. Math., vol. 92, Birkhäuser Boston, Boston, MA, 1990, pp. 85-124.

[51] N. N. Lebedev, Special functions and their applications, Dover Publications Inc., New York, 1972.

[52] J.-S. Li, Minimal representations $\& 5$ reductive dual pairs, Representation theory of Lie groups (Park City, UT, 1998), IAS/Park City Math. Ser., vol. 8, Amer. Math. Soc., Providence, RI, 2000, pp. 293-340.

[53] P. Macaulay-Owen, Parseval's theorem for Hankel transforms, Proc. London Math. Soc. 45 (1939), 458-474.

[54] G. Mano, Radon transform of functions supported on a homogeneous cone, preprint.

[55] C. S. Meijer, On the G-function. I-VIII, Nederl. Akad. Wetensch., Proc. 49 (1946), 227-237; 344-356; 457-469; 632-641; 765-772; 936-943; $1063-1072 ; 1165-1175$.

[56] P.-D. Methée, Systèmes différentiels du type de Fuchs en théorie des distributions, Comment. Math. Helv. 33 (1959), 38-46.

[57] W. Myller-Lebedeff, Die Theorie der Integralgleichungen in Anwendung auf einige Reihenentwicklungen, Math. Ann. 64 (1907), 388-416.

[58] G. I. Ol'shanskil, Complex Lie semigroups, Hardy spaces and the Gel'fand-Gindikin program, Differential Geom. Appl. 1 (1991), 235246. 
[59] P. Perrin, Représentations de Schrödinger, indice de Maslov et groupe métaplectique, Noncommutative harmonic analysis and Lie groups (Marseille, 1980), Lecture Notes in Math., vol. 880, Springer, Berlin, 1981, pp. 370-407.

[60] A. P. Prudnikov, Yu. A. Brychkov, and O. I. Marichev, Integrals and series. Vol. 3: More special functions, Gordon and Breach Science Publishers, New York, 1990.

[61] S. Rallis and G. Schiffmann, Weil representation. I. Intertwining distributions and discrete spectrum, Mem. Amer. Math. Soc. 25 (1980), no. 231.

[62] H. Sabourin, Une représentation unipotente associée à l'orbite minimale: le cas de $\mathrm{SO}(4,3)$, J. Funct. Anal. 137 (1996), 394-465.

[63] S. Sahi, Explicit Hilbert spaces for certain unipotent representations, Invent. Math. 110 (1992), 409-418.

[64] W. Schmid and K. Vilonen, Characteristic cycles and wave front cycles of representations of reductive Lie groups, Ann. of Math. (2) 151 (2000), 1071-1118.

[65] T. A. Springer, Jordan algebras and algebraic groups, Springer-Verlag, New York, 1973.

[66] R. J. Stanton, Analytic extension of the holomorphic discrete series, Amer. J. Math. 108 (1986), 1411-1424.

[67] E. M. Stein, Analysis in matrix spaces and some new representations of $\operatorname{SL}(N, \mathbb{C})$, Ann. of Math. (2) 86 (1967), 461-490.

[68] E. M. Stein and G. Weiss, Introduction to Fourier analysis on Euclidean spaces, Princeton University Press, Princeton, N.J., 1971.

[69] M. Takeuchi, Modern spherical functions, Translations of Mathematical Monographs, vol. 135, American Mathematical Society, Providence, RI, 1994.

[70] M. E. Taylor, Partial differential equations. I, Applied Mathematical Sciences, vol. 115, Springer-Verlag, New York, 1996.

[71] E. C. Titchmarsh, Introduction to the theory of Fourier integrals, Oxford University Press, Oxford, 1937; third ed., Chelsea Publishing Co., New York, 1986. 
[72] P. Torasso, Quantification géométrique, opérateurs d'entrelacement et représentations unitaires de $(\widetilde{\mathrm{SL}})_{3}(\mathbf{R})$, Acta Math. 150 (1983), 153242.

[73] P. Torasso, Méthode des orbites de Kirillov-Duflo et représentations minimales des groupes simples sur un corps local de caractéristique nulle, Duke Math. J. 90 (1997), 261-377.

[74] M. Vergne and H. Rossi, Analytic continuation of the holomorphic discrete series of a semi-simple Lie group, Acta Math. 136 (1976), 1-59.

[75] D. A. Vogan, Jr., Gel'fand-Kirillov dimension for Harish-Chandra modules, Invent. Math. 48 (1978), 75-98.

[76] D. A. Vogan, Jr., Singular unitary representations, Noncommutative harmonic analysis and Lie groups (Marseille, 1980), Lecture Notes in Math., vol. 880, Springer, Berlin, 1981, pp. 506-535.

[77] D. A. Vogan, Jr., Unitary representations of reductive Lie groups, Annals of Mathematics Studies, vol. 118, Princeton University Press, Princeton, NJ, 1987.

[78] N. R. Wallach, Real reductive groups. I, II, Pure and Applied Mathematics, vol. 132, Academic Press Inc., Boston, MA, 1988, 1992.

[79] G. N. Watson, A treatise on the theory of Bessel functions, Cambridge University Press, Cambridge, 1922.

[80] C.-B. Zhu and J.-S. Huang, On certain small representations of indefinite orthogonal groups, Represent. Theory 1 (1997), 190-206.

Toshiyuki Kobayashi

Home address: Graduate School of Mathematical Sciences, the University of Tokyo, 3-8-1 Komaba, Meguro, Tokyo, 153-8914, Japan

E-mail address: toshi@ms.u-tokyo.ac.jp

Current address: Department of Mathematics, FAS, Harvard University, One Oxford Street, Cambridge MA 02138 USA

Gen Mano

Graduate School of Mathematical Sciences, the University of Tokyo, 3-8-1

Komaba, Meguro, Tokyo, 153-8914, Japan

E-mail address: gmano@ms.u-tokyo.ac.jp 


\section{List of Symbols}

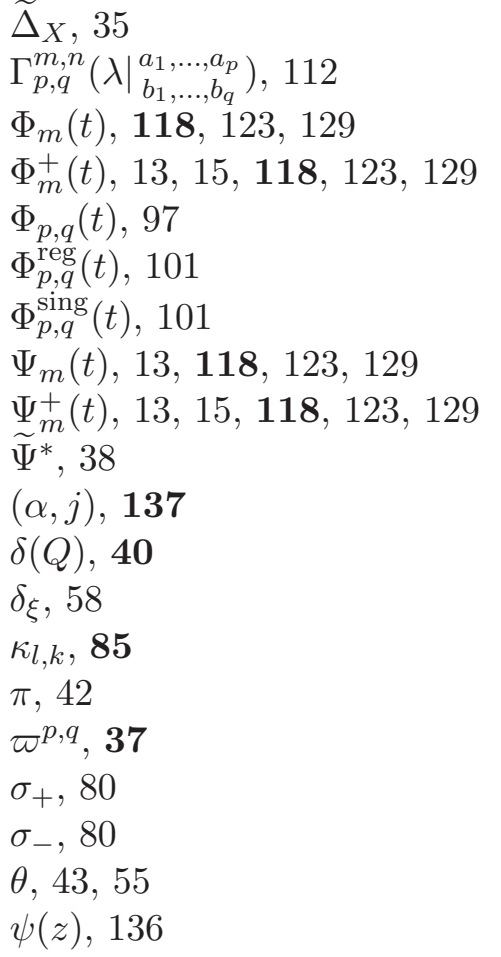

Ap1-Ap5, $149-150$

B1-B3, 137-138

C1-C4, $19-20$

F1-F8, 7

Ge1-Ge4, 140-141

H1-H6, 141-142

I0-I5, 55,56

K1-K8, $9-10$

L1-L2, 139

M1-M5, 21

P1-P5, 6

S1-S2, 67-68

$\square, 6,46,49,51$
$\sum_{\widehat{\otimes}, 30}^{\square}, 30$
A, 44

$\mathfrak{a}, 44$

$A_{\kappa}, 82$

$\operatorname{Ann}(\pi), 17$

$A_{q}^{m, 0}, 147$

$\bar{A}_{q}^{m, 0}, 147$

$C, 4$

$\widetilde{C}, 44,126$

$C_{0}^{\infty}(C), 11,100$

$C_{0}^{k}(\mathbb{R}), 118,132$

$C_{l}^{\mu}(x), 139$

$\widetilde{C}_{l}^{\mu}(x), 103,139$

$C O(Q), 5,47,56$

$\operatorname{DIM} \pi, 17$

$E, 5,46,53$

$f_{l, k}(r), 65,69,83,94$

$F_{1}\left(\alpha, \beta, \beta^{\prime}, \gamma ; x, y\right), 149$

$F_{2}\left(\alpha, \beta, \beta^{\prime}, \gamma, \gamma^{\prime} ; x, y\right), \mathbf{1 4 9}$

$F_{3}\left(\alpha, \alpha^{\prime}, \beta, \beta^{\prime}, \gamma ; x, y\right), \mathbf{1 4 9}$

$F_{4}\left(\alpha, \beta, \gamma, \gamma^{\prime} ; x, y\right), 149$

${ }_{p} F_{q}, 145$

$\mathcal{F}_{C}$, 9, 55, 56, 59, 98, 102

$\mathcal{F}_{\mathbb{R}^{n}}, 7$, 20, 29, 30, 42, 58, 76, 99 , 102

$G\left(x_{+}\right)_{L}, \mathbf{1 1 3}$

$G_{p, q}^{m, n}\left(\left.x_{+}\right|_{b_{1}, \ldots, b_{q}} ^{a_{1}, \ldots, a_{p}}\right)_{L}, 113$

$G_{p, q}^{m, n}\left(\left.x\right|_{b_{1}, \cdots, b_{q}} ^{a_{1}, \cdots, a_{p}}\right), 144$

$H, 43,55$

$\mathcal{H}^{j}\left(\mathbb{R}^{m}\right)$, 30, 38, 65, 76, 102, 141

$H_{l, k}, 65,67,94$

$I_{i \rightarrow j}^{m}: \mathcal{H}^{i}\left(\mathbb{R}^{m-1}\right) \rightarrow \mathcal{H}^{j}\left(\mathbb{R}^{m}\right), 66$, 


$$
\begin{aligned}
& I_{\nu}(z), 133 \\
& \widetilde{I}_{\nu}(z), \mathbf{1 3 4} \\
& J_{\nu}(z), 133 \\
& \widetilde{J}_{\nu}(z), 119,134,135 \\
& K \simeq O(p) \times O(q), 43,65 \\
& K^{\prime} \simeq O(p-1) \times O(q-1), 43,65 \text {, } \\
& 107 \\
& K_{l, k}(t), 31,75,86,107 \\
& K_{\nu}(z), 65,133 \\
& \widetilde{K}_{\nu}(z), \text { 65, 119, 134, } 135 \\
& K\left(x, x^{\prime}\right), 12,97 \\
& L^{2}(C), \mathbf{5}, 30,41 \\
& L^{2}(C)^{\infty}, 45,57 \\
& L^{2}(C)_{K}, 45,67 \\
& L^{2}(C)^{-\infty}, 9,57 \\
& m_{0}, 43 \\
& M^{\max } \simeq O(p-1, q-1) \times \mathbb{Z}_{2}, 43 \\
& M_{+}^{\max } \simeq O(p-1, q-1), 43 \\
& M p(n, \mathbb{R}), 19 \\
& M \simeq S^{p-1} \times S^{q-1}, 36 \\
& M_{+}, 38,41 \\
& n_{a}, 44 \\
& \bar{n}_{a}, 44 \\
& N_{j}, 43 \\
& \bar{N}_{j}, 43 \\
& \frac{N^{\max }, 44}{N^{\max }}, 44 \\
& \mathfrak{n}^{\max }, 44,55 \\
& \overline{\mathfrak{n}^{\max }}, 44,55 \\
& O(p, q), 20,37 \\
& \mathcal{O}_{\min }, 19,42 \\
& \mathcal{O}_{\text {min }}^{\mathbb{C}}, 17 \\
& P_{j}, 5, \text { 47, 54, 59, } 128 \\
& P_{j}(b), 46,50,53 \\
& \overline{P^{\max }}:=M^{\max } A \overline{N^{\max }}, 23,44 \\
& P_{\nu}^{\mu}(x), \mathbf{1 3 8} \\
& P_{\text {Siegel }}, 20 \\
& Q(x), 40 \\
& Q(x)_{+}^{\lambda}, 49 \\
& \mathcal{R}, 10,100 \\
& \mathbb{R}^{p, q}, 36 \\
& \mathbb{R}\left[x, \frac{\partial}{\partial x}\right],[5 \\
& \mathbb{R}\left[x, \frac{\partial}{\partial x}\right]^{C},[5,54 \\
& \mathcal{S}^{\prime}, \text { 81, } 90 \\
& \mathcal{S}=\sigma_{-}^{-1}(\mathcal{S}(\mathbb{R})), \underline{81}, 90 \\
& \mathcal{S}\left(\mathbb{R}^{n}\right), 8,80 \\
& \mathcal{S}^{\prime}\left(\mathbb{R}^{n}\right), 8,41,80 \\
& \operatorname{Sol}\left(\widetilde{\Delta}_{S^{n_{1}} \times S^{n_{2}}}\right), 22,40 \\
& \operatorname{Sol}\left(\widetilde{\Delta}_{X}\right), 36 \\
& \operatorname{Sol}\left(\square_{\mathbb{R}^{n_{1}, n_{2}}}\right), 22,40 \\
& S p(n, \mathbb{R}), 19 \\
& \mathfrak{s p}(n, \mathbb{R}), 11 \\
& \mathcal{T}: L^{2}(C) \rightarrow \overline{V^{p, q}}, 41,66 \\
& T: L^{2}(C) \rightarrow \mathcal{S}^{\prime}\left(\mathbb{R}^{n}\right), 41,49 \\
& T_{l, k}, 30, \text { 75, 85, } 107 \\
& V^{p, q}, \mathbf{3 7} \\
& w_{0}, 23,55 \\
& w_{0}^{\prime}, 20,76,99 \\
& x_{+}^{\lambda}, 15,130 \\
& x^{-k}, 132 \\
& x_{+}^{-k}, 132 \\
& Y_{\nu}(z), 133 \\
& \widetilde{Y}_{\nu}(z), 119,134,135
\end{aligned}
$$




\section{Index}

Appell's hypergeometric function, 29, 32, 138, 149

associated Legendre function, $\mathbf{1 3 8}$, 141

associated variety, 17, 18

asymptotic behavior

$$
\begin{aligned}
& -, f_{l, k}, 70 \\
& -, \sigma_{-}\left(f_{l, k}\right), 83 \\
& -, K_{l, k}, 86 \\
& -, \Gamma_{p, q}^{m, n}\left(\lambda \mid \begin{array}{c}
a_{1}, \ldots, a_{p} \\
b_{1}, \ldots, b_{q}
\end{array}\right), 114 \\
& -, \text { Bessel distribution, } 13 \\
& -, \text { Bessel function, } 136 \\
& -, \text { G-function, } 146 \\
& -, \text { gamma function, } 89
\end{aligned}
$$

asymptotic support, 18

backward light cone, 14

Barnes generalized hypergeometric function, 145

Bessel differential equation, 133

Bessel distribution, 12, 97, 122

—, differential equation, 129

—, integral expression, 117

-, Laurent series expansion, 15

Bessel function

—, of the first kind, 133

-, of the second kind, 133

bipolar coordinate, 30

boundary value, 25

Cartan involution, 43, 55

Cartan-Helgason theorem, 28

Cauchy's principal value, 132

cohomological induction, 16

conformal compactification, 38

conformal inversion, $23, \mathbf{5 6}$ conformal model, 3, 22, 35, 150

conserved quantity, 27

differentiable vector, 45

Dirac delta function, 40, 58, 131

discretely branching law, 28

distribution vector, $\mathbf{5 7}$

Euclidean Fourier transform, 7, 25, 29, 102

Euler operator, 5, 46

Euler vector field, 53

forward light cone, 14

Fourier-Hankel transform, 27

Fox's reciprocal formula, 77

fundamental differential operator, 2, 5, 47, 128

Funk-Hecke formula, 104, 109, 152

Gauss's duplication formula, 139

Gegenbauer polynomial, 32, 103 ,

$$
\text { 139, } 142
$$

Gelfand triple, 57

Gelfand-Gindikin program, 25

Gelfand-Kirillov dimension, 17

generalized function, 59

geometric quantization, 19

Green function, 27

Hankel transform, 2, 30, 31, 76,

$$
\text { 138, } 151
$$

Hankel's notation, 137

harmonic representation, 19

Heisenberg Lie algebra, 11

Hermite subgroup, 25

highest weight module, 21, 24, 67,

75, 78

holomorphic semigroup, 25, 99 
I-Bessel function, 134

indefinite orthogonal group, 20

indefinite orthogonal Lie algebra, 11

integral of Euler's type, 150

intrinsic inner product, 27

isotropic cone, 4, 14

$J$-Bessel function, 134

Jordan algebra, 56

$K$-Bessel function, 29, 32, 134

$K$-picture, 22, 34

Kantor-Koecher-Tits group, 56

Knapp-Stein intertwining operator, 28

Kostant-Sekiguchi correspondence, 18

$L^{2}$-model, 26, 150

ladder representation, 32

Laplace-Beltrami operator, 2, 35

light cone, 14

Mehler kernel, 25

Meijer's G-distribution, 111

Meijer's $G$-function, 29, 31, 144

Meijer's $G$-transform, 31

Mellin transform, 102

-, of Bessel function, 137

Mellin-Barnes type integral, 113.

118, 144

metaplectic group, 19

minimal representation, 2, 17, 21,

39

Minkowski space, 24

Möbius transform, 23

modified Bessel function

-, of the first kind, 133

- , of the third kind, 134

multiplicity free, 27 multiplicity-free decomposition, 102

$N$-picture, 22, 34, 151

oscillator representation, 19

Parseval type formula, 27

Plancherel formula

-, for Meijer's $G$-transform, 31

一, $\mathcal{F}_{C}, 24,98$

—, $G$-function, 77

Radon transform, 10, 100, 102

reciprocal formula, 24

-, for Meijer's G-transform, 31

一, $\mathcal{F}_{C}, 24,98$

—, G-function, 77

Riesz distribution, $\mathbf{1 3 0}$

Riesz potential, 104

Schrödinger, 99

Schrödinger model, 3, 19, 20, 22,

42, 150

Segal-Shale-Weil representation, 19

Siegel parabolic subgroup, 20

smooth vector, 45

solution model, 26

spherical harmonics, 30, 141

spherical representation, $\mathbf{2 1}$

symplectic group, 19

symplectic Lie algebra, 11

tempered distribution, 22, 25, 80

theta correspondence, 21, 39

ultra-hyperbolic equation, 11, 22

uncertainty principle, 27

unitary inversion operator, 10,27 ,

59, 97 
wave front set, 18

Weil representation, 3, 11, 19, 42,

76, 99

Weyl algebra, 5, 47

Yamabe operator, 22, 35

$Y$-Bessel function, 134 